University of Louisville

ThinkIR: The University of Louisville's Institutional Repository

Electronic Theses and Dissertations

$12-2019$

\title{
Development and characterization of a biopolymer direct-write process for 3D microvascular structures formation.
}

Xiaoming Fan

University of Louisville

Follow this and additional works at: https://ir.library.louisville.edu/etd

Part of the Biomechanical Engineering Commons

\section{Recommended Citation}

Fan, Xiaoming, "Development and characterization of a biopolymer direct-write process for 3D microvascular structures formation." (2019). Electronic Theses and Dissertations. Paper 3335.

https://doi.org/10.18297/etd/3335

This Doctoral Dissertation is brought to you for free and open access by ThinkIR: The University of Louisville's Institutional Repository. It has been accepted for inclusion in Electronic Theses and Dissertations by an authorized administrator of ThinkIR: The University of Louisville's Institutional Repository. This title appears here courtesy of the author, who has retained all other copyrights. For more information, please contact thinkir@louisville.edu. 


\title{
DEVELOPMENT AND CHARACTERIZATION OF A BIOPOLYMER DIRECT-WRITE PROCESS FOR 3D MICROVASCULAR STRUCTURES FORMATION
}

\author{
By \\ Xiaoming Fan \\ B.E., Beijing University of Chemical Engineering, 2007 \\ M.S., University of Louisville, 2014

\begin{abstract}
A Dissertation
Submitted to the Faculty of the

J.B. Speed School of Engineering of the University of Louisville

in Partial Fulfillment of the Requirements

for the Degree of
\end{abstract}

Doctor of Philosophy in Mechanical Engineering

Department of Mechanical Engineering

University of Louisville

Louisville, Kentucky

December 2019 
Copyright 2019 By Xiaoming Fan

All rights reserved 



\title{
DEVELOPMENT AND CHARACTERIZATION OF A BIOPOLYMER DIRECT-WRITE PROCESS FOR 3D MICROVASCULAR STRUCTURES FORMATION
}

\author{
By \\ Xiaoming Fan \\ B.E., Beijing University of Chemical Engineering, 2007 \\ M.S., University of Louisville, 2014 \\ A Dissertation Approved on
}

June 21, 2019

by the following Dissertation Committee:

Dr. Robert Keynton, Dissertation Director

Dr. Stuart Williams, Dissertation Co-Director

Dr. Patricia Soucy

Dr. Kunal Kate

Dr. Gamini Sumanasekera 


\section{DEDICATION}

This dissertation is dedicated to my mom (Fei Peng) in the heaven. 


\section{ACKNOWLEDGMENTS}

I would like to first thank Dr. Robert Keynton for being my research advisors, as well as life mentors for five years. Since my first day in his group, he believed in me and gave me endless support. On the academic level, I learned how to define a problem, analyze and find a solution under his guidance. On the personal level, Dr. Keynton taught me how to be a humble, sincere and honest person. These are all lifetime inspirations that will stay with me forever.

I would like to thank Dr. Stuart Williams as my co-advisor. He was always being there when I need help. His knowledge and personality undoubtedly helped me to grow into a real researcher/engineer. I would like also to present my sincere thanks to Dr. Patricia Soucy. I have learned so much from her as I have zero background in cell culture at the beginning. She was always the one that I like to talk to for my research and life. She not only taught me what she knows but also willing to learn and discuss with me together when we encountered problems. Special thanks to Dr. Kunal Kate for helping me to set up the polymerization experiments and all his insight throughout this project. Last but not least, many thanks to Dr. Gamini Sumanasekera for his contributions and participating in my dissertation defense.

I am grateful to the former and present group members of Dr. Keynton's research group: Dr. Mark Crain, Dr. Scott Cambron, Dr. Rajat Chauhan, Mr. Hares 
Patel, Dr. Thomas Roussel, Dr. Hanwen Yuan. I am also appreciative of all the faculty, staff and friends in Mechanical Engineering, Bio-Engineering, and Chemistry departments that offer me helps. Special thanks to Mr. Nicholas Huffman, Mr. Doug Jackson, Dr. Zhichao Lu, Ms. Betty Nunn, Dr. Matin O'Toole, and Ms. Larissa Pack.

Finally, I would like to thank my wife and best friend, Dr. Wuyu Zhang and my lovely son, Samuel Fan. Without their unconditionally support, I will never achieve this goal. 


\begin{abstract}
DEVELOPMENT AND CHARACTERIZATION OF A BIOPOLYMER DIRECTWRITE PROCESS FOR 3D MICROVASCULAR STRUCTURES FORMATION

Xiaoming Fan

June 21, 2019
\end{abstract}

Engineering of bulk tissues has been limited by the lack of nutrient and waste exchange in these tissues without an adjacent capillary network. To produce microvasculature, a scaffold must be produced that provides temporary mechanical support and stimulate endothelial cell adhesion, growth, and morphogenesis into a vessel. However, current well-established techniques for producing microvasculature, such as electrospinning, are limited since they lack both the precision to control fiber placement in three-dimensional space and the ability to create fiber networks with predefined diameters to replicate the physiological microvascular progression from arteriole to capillary to venule. Our group has developed a "Direct-write" technique using a 3-Axis robotic dispensing system to process polymers into precisely positioned, three-dimensional, suspended fibers with controlled diameters. 
Within this dissertation, a conceptual scaffold-covering strategy is presented for the formation of the precisely positioned, three-dimensional microvascular structure with a controlled diameter in vitro. This study considers ways to extend the 3-Axis robotic dispensing system by incorporating new biodegradable materials into micro-fibers. First, a number of different biopolymers (natural, synthetic, composites, and copolymers) were used for demonstrating the capability of direct-writing micro-fibers and branched structures with microvascular-scale diameter through the 3-Axial robotic dispensing system. Then, the fabrication process was characterized by a design of experiments and a generalized mathematical model was developed through dimensional analysis. The empirical model determined the correlation between polymer fiber diameter and intrinsic properties of the polymer solution together with the processing parameters of the robotic dispensing system and allows future users the ability to employ the 3-Axis robotic dispensing system to direct-write micro-fibers without trial-and-error work. This study also considers ways to broaden the prevascularization methods by covering Human Dermal Microvascular Endothelial Cells (HDMECs) on the fabricated scaffold to generate the microvascular structure. HDMECs cultured on the produced micro-fiber scaffolds were observed to form a confluent monolayer spread along the axis and around the circumference of the fibers within two days of seeding. Once confluency was reached, the cell-covered scaffold was embedded into a collagen gel and a hybrid structure was formed. Through these experiments, we demonstrate the ability to obtain a cell-viable, 
flexible, and free-standing "modular tissue", which could be potentially assembled to a three-dimensional microvascular network through angiogenesis mechanism. 


\section{TABLE OF CONTENTS}

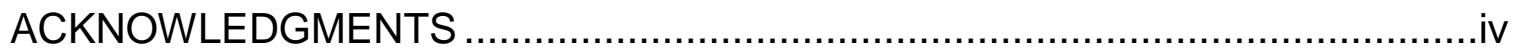

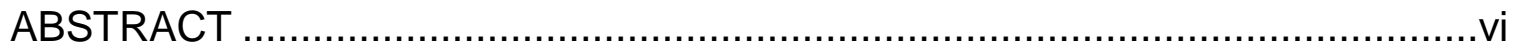

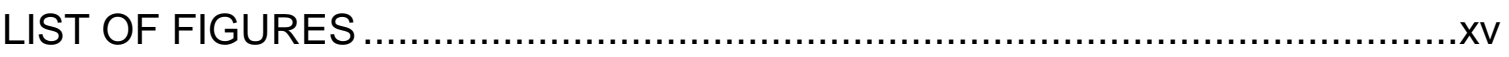

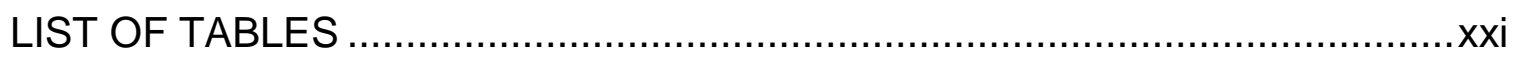

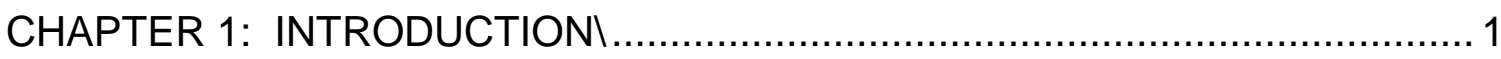

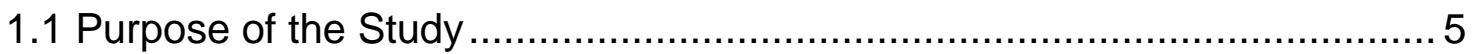

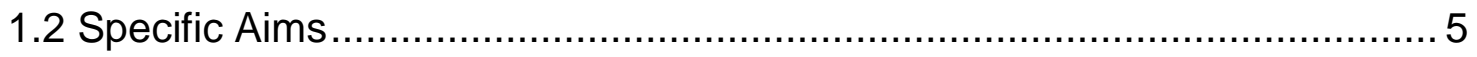

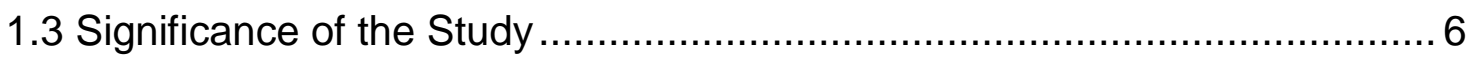

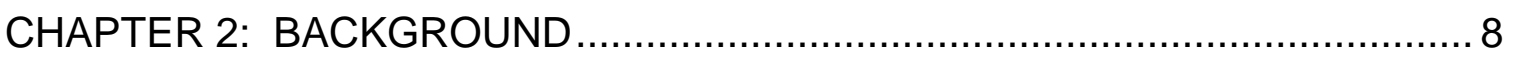

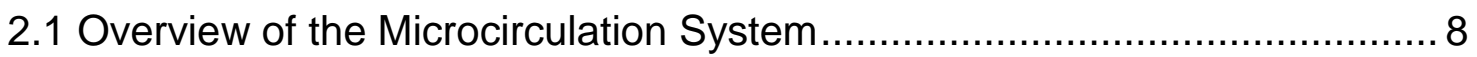

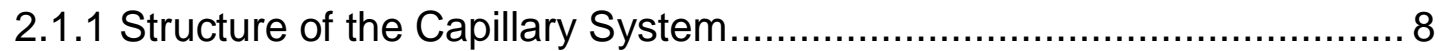

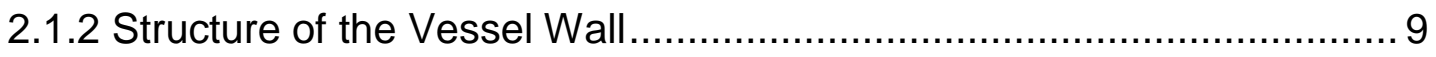

2.1.3 Structure of the Capillary Wall..................................................... 11

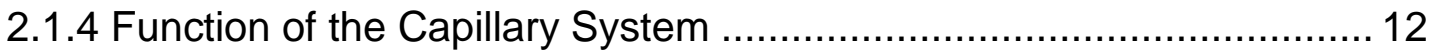

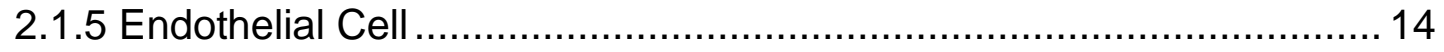

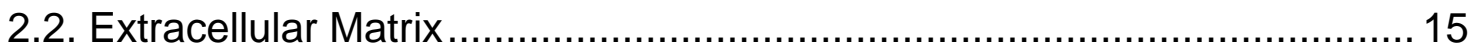

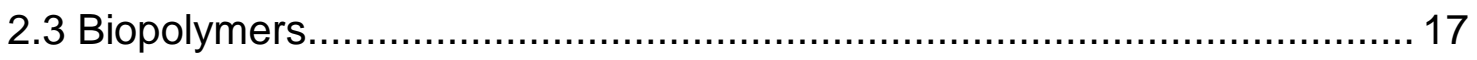




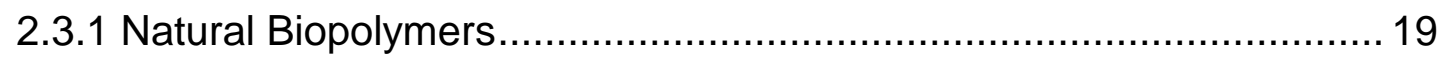

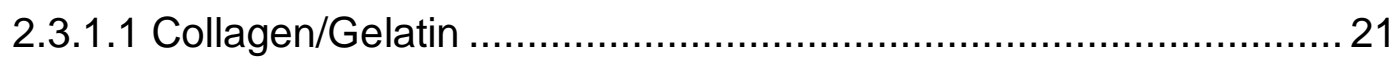

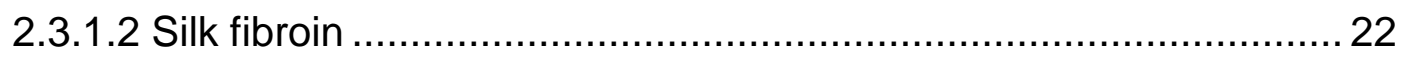

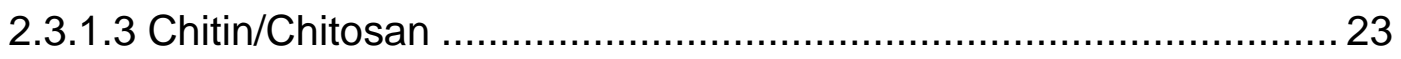

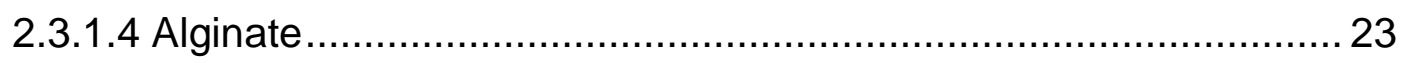

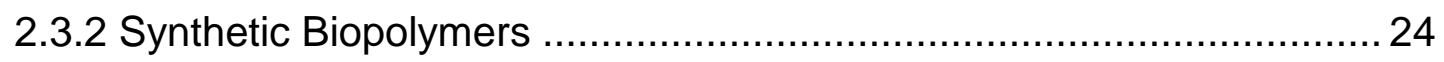

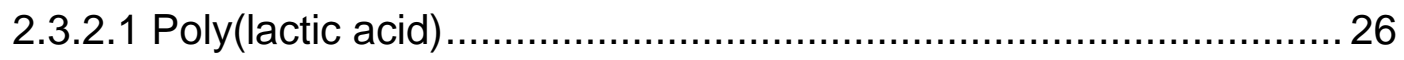

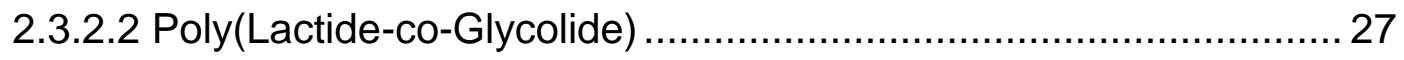

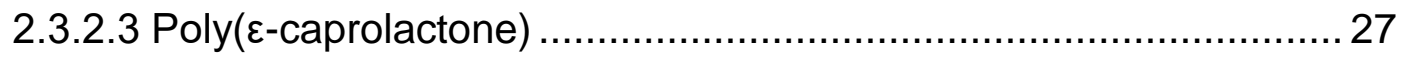

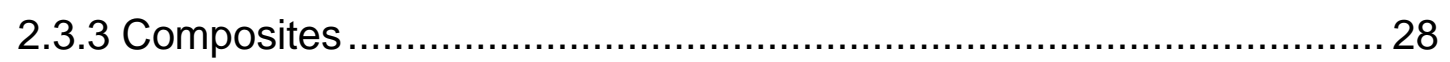

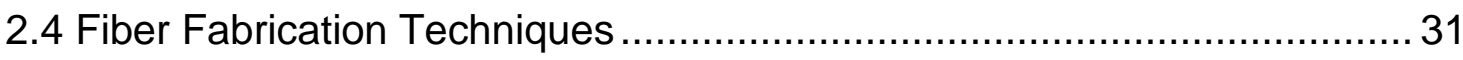

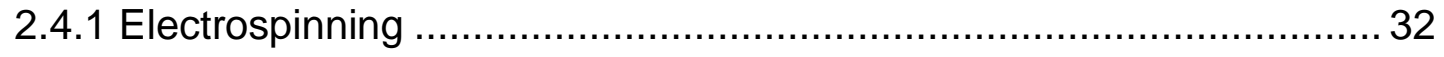

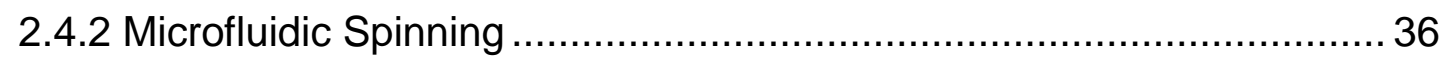

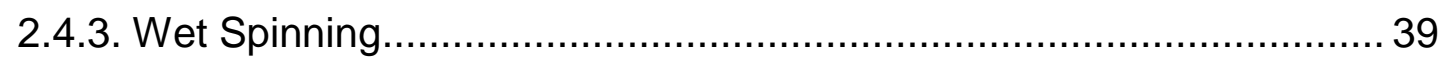

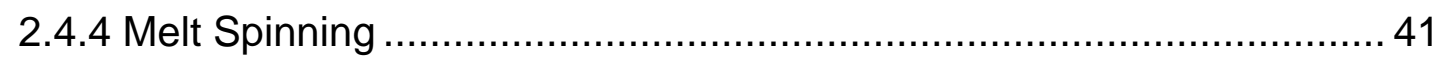

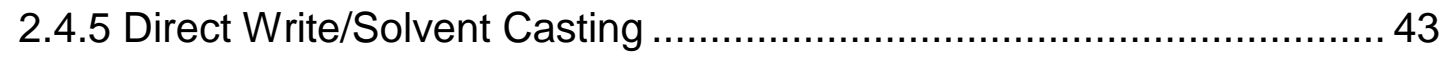

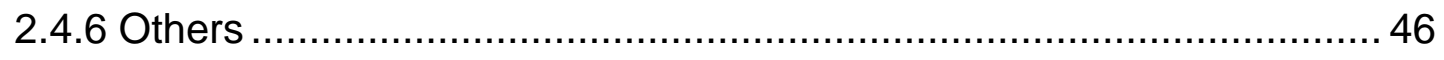

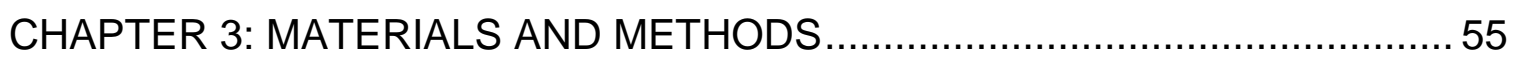

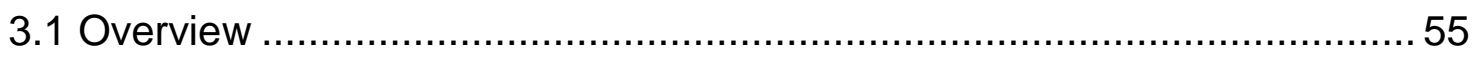

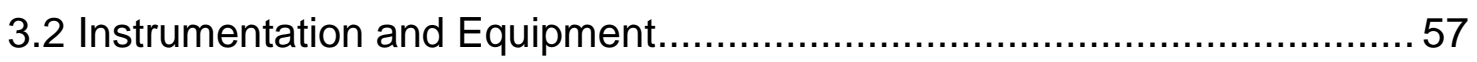


3.2.13-Axis Robotic Dispensing System

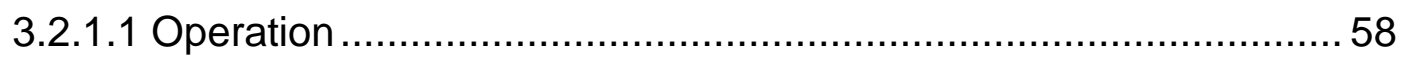

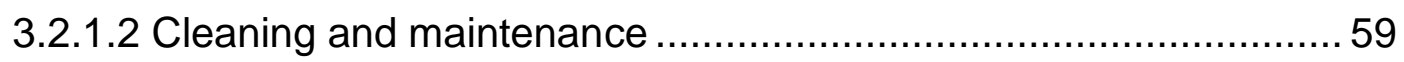

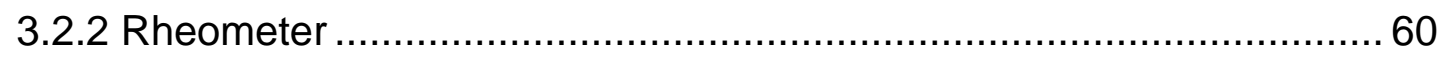

3.2.3 Du Nüoy-Paddy Surface Tension Measurement System .................... 62

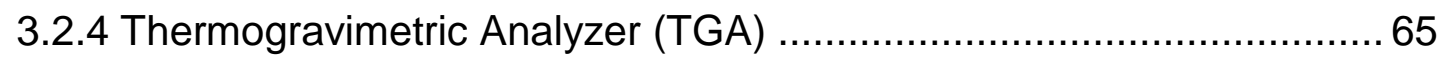

3.2.5 Fourier Transform Infrared Spectrometer (FT-IR) ............................ 67

3.2.6 Nuclear Magnetic Resonance (NMR) Spectroscopy ...........................6 68

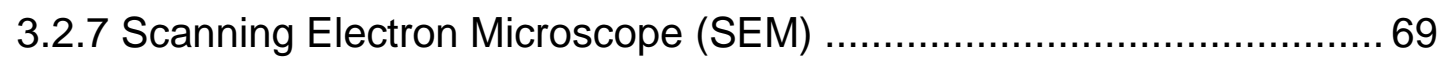

3.2.8 Laser Cutting System............................................................. 71

3.2.9 Cell Culture Related Equipment.................................................. 73

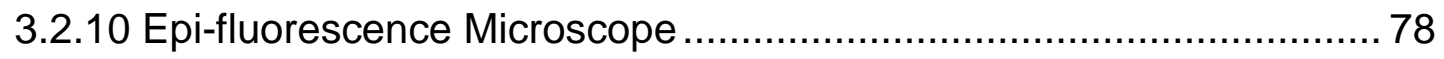

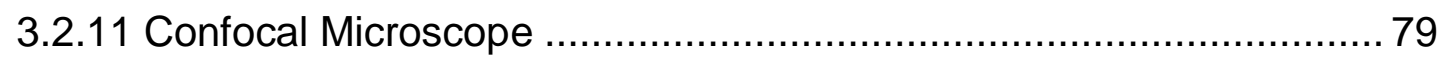

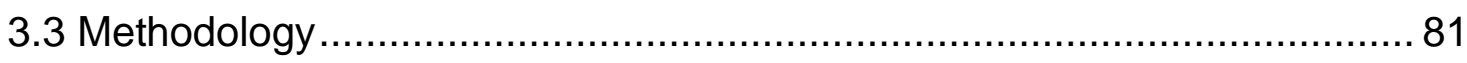

3.3.1 Biopolymer Solution Preparation................................................ 81

3.3.1.1 Gelatin, PLGA, and Composites' Solution ............................... 81

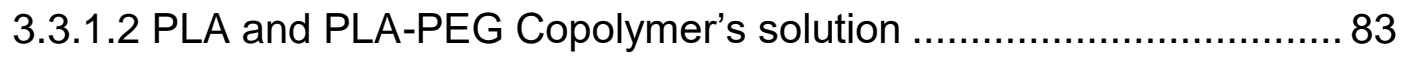

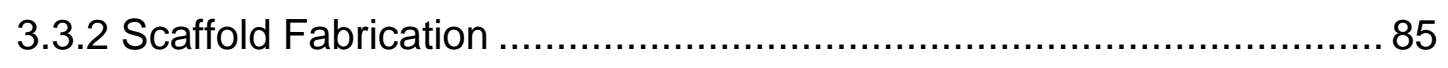

3.3.2.1 Fabrication of Single Suspended Fiber ...................................... 85

3.3.2.2 Initial Polymer Drop Size and Needle-to-Substrate Gap............... 86 
3.3.2.3 Fabrication of Branched Structure 86

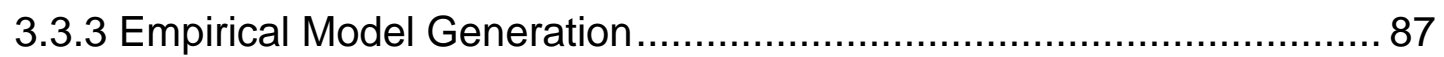

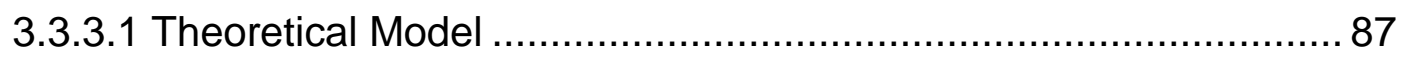

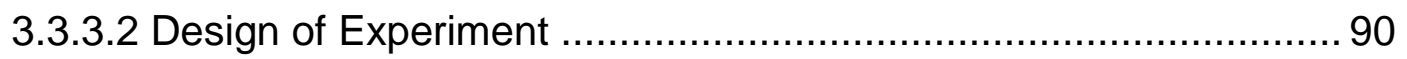

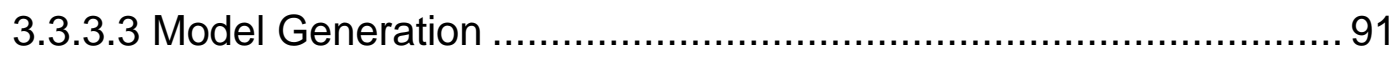

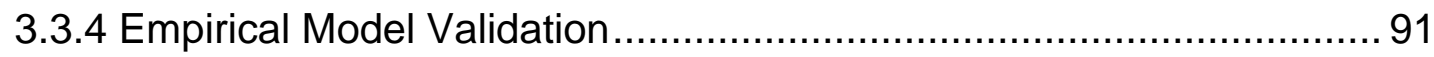

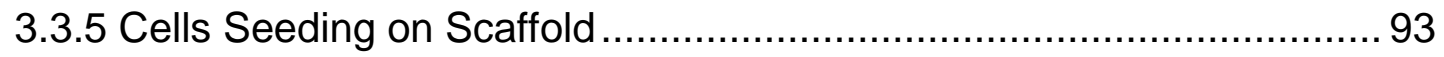

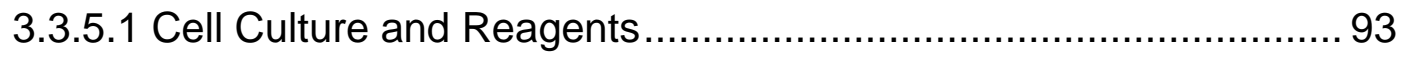

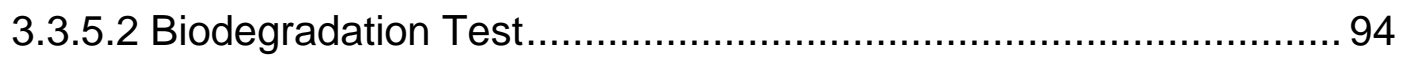

3.3.5.3 Biopolymers Cellular Adhesion/Viability Test..............................95

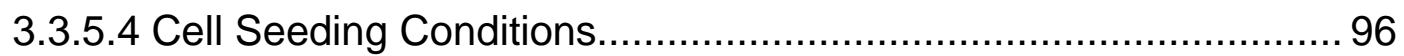

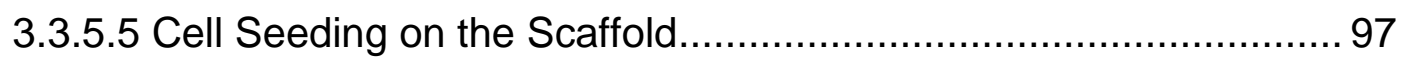

3.3.5.6 Immunofluorescent Staining .................................................. 99

3.3.5.6.1 DAPI and Rhodamine Phalloidin Staining ............................ 99

3.3.5.6.2 VE-Cadherin Staining ...................................................... 100

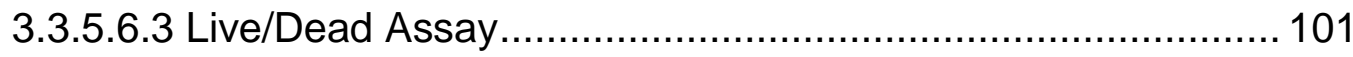

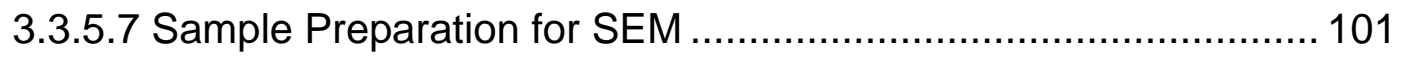

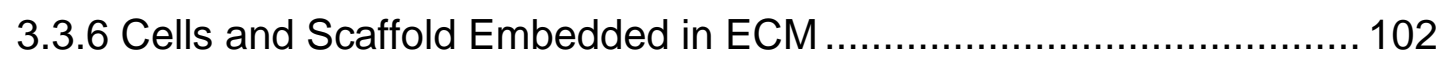

3.3.6.1 Collagen Gel Solution Preparation.......................................... 102

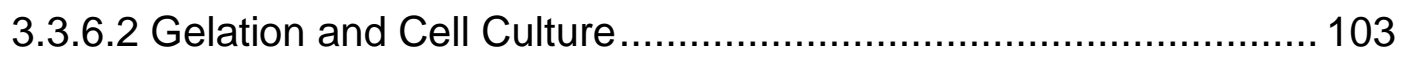


3.3.6.3 Imaging Samples Preparation................................................ 104

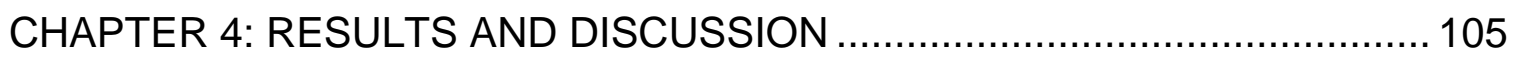

4.1 Fabrication of Biopolymer Micro-fibers and Branch Structures via 3-Axis

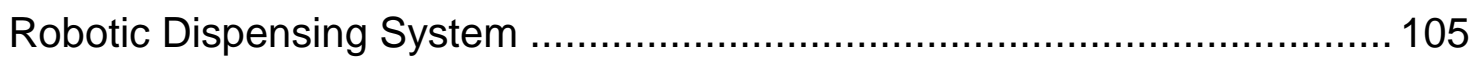

4.1.1 Gelatin, PLGA, and Composites ................................................ 106

4.1.2 PLA and PLA-PEG Copolymers................................................. 113

4.2 Characterization and Modeling of the Direct-write Process ................... 123

4.2.1 Characterization of the Direct-write Process .................................... 124

4.2.1.1 Process Factors Reduction .................................................... 124

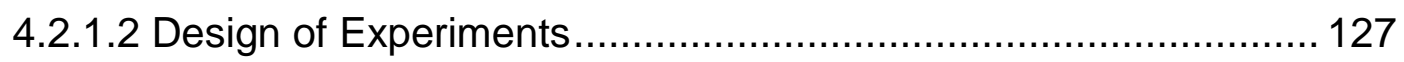

4.2.2 Generation of the Empirical Model .............................................. 132

4.2.2.1 Characterization of Gelatin Solution ..................................... 132

4.2.2.2 Dimensional Analysis for Empirical Model Generation ............... 134

4.2.3 Validation of the Empirical Equation ........................................... 140

4.3 Direct-write Microvascular Scaffold for Endothelial Cells Morphogenesis 147

4.3.1 Biopolymer Selection for Scaffold ............................................... 147

4.3.1.1 Biodegradation Test......................................................... 147

4.3.1.2 Biopolymers Cellular Adhesion and Viability Test...................... 149

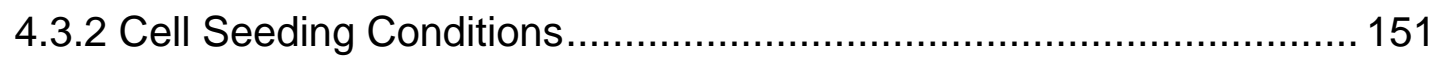

4.3.2.1 BSA Coating Experiment ................................................... 151 
4.3.2.2 Adhesion Promoters Comparison ............................................ 152

4.3.2.3 Cell Seeding Density Experiment ............................................. 154

4.3.3 Endothelial Cell Tubulogenesis on Microvascular Scaffold ............... 155

4.4. Prevascularization of Hybrid Constructs ............................................ 162

CHAPTER 5: CONCLUSIONS AND FUTURE DIRECTIONS ....................... 172

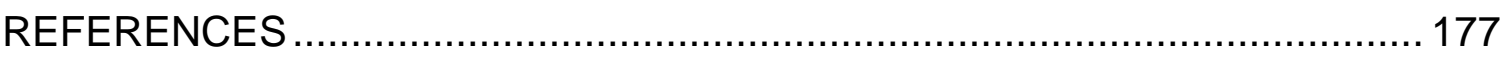

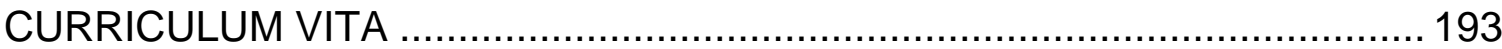




\section{LIST OF FIGURES}

Figure 2. 1 Illustration of the structure of the capillary bed .............................. 9

Figure 2. 2 Illustration of the structure of the capillary wall .............................. 11

Figure 2. 3 Illustration of three structures of capillaries: continuous capillary, fenestrated capillary, and sinusoid capillary ............................................... 12

Figure 2. 4 Diffusion of fluid molecules between the capillary and interstitial fluid

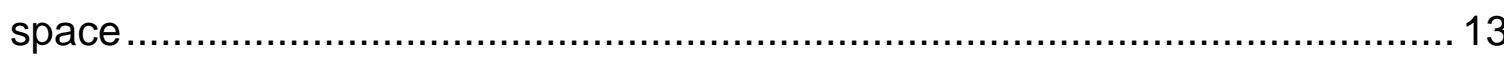

Figure 2. 5 Examples of common proteoglycans and fibrous proteins ............. 17

Figure 2. 6 Classification of several common natural biopolymers .................... 20

Figure 2. 7 Collagen triple helical structure and gelatin coil structure ................ 22

Figure 2. 8 Classification of several common synthetic biopolymers................ 25

Figure 2. 9 Schematic diagram of set up of electrospinning ............................. 33

Figure 2. 10 Schematic diagram of set up of microfluidic spinning ................... 37

Figure 2. 11 Schematic diagram of set up of wet spinning ............................. 40

Figure 2. 12 Schematic diagram of set up of melt spinning ............................ 42

Figure 2. 13 Schematic diagram of set up of direct write ................................. 45

Figure 3. 1 Schematic illustrating of the concept of the formation of a

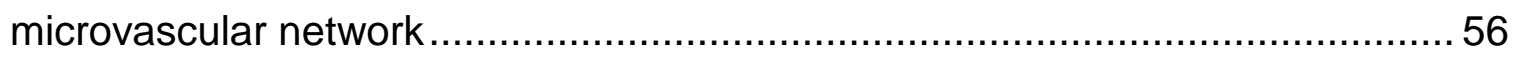

Figure 3. 2 Optical Images of the 3-Axis robotic dispensing systems ................ 58

Figure 3. 3 Graphic interface of JR C-Points software ................................... 59 
Figure 3. 4 Image of disassembling components of EFD 741 dispense valve.... 60 Figure 3. 5 Image of Physica MCR 300 rheometer under testing ...................... 61

Figure 3. 6 The viscosity of standard fluid for calibration .................................. 62

Figure 3. 7 Image of custom Du Nüoy-Paddy surface tension measurement

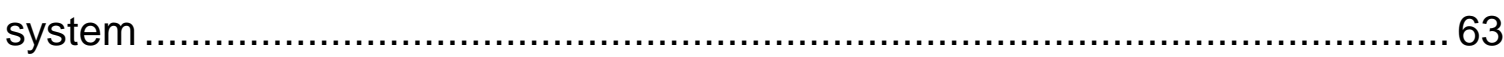

Figure 3. 8 Calibration of the linear actuator length corresponding the LabView

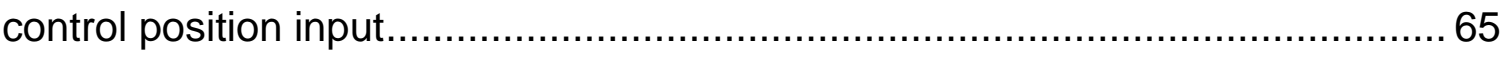

Figure 3. 9 Image of TA SDT Q600 Simultaneous TGA/DSC ........................... 65

Figure 3. 10 Image of QSeries software graphics interface under testing .......... 66

Figure 3. 11 Image of PerkinEImer Spectrum 100 FT-IR spectrometer ............... 67

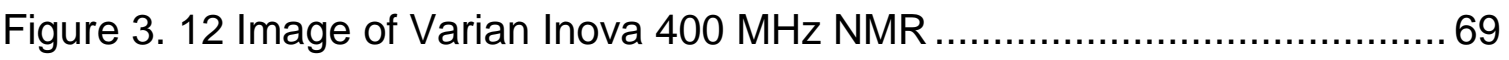

Figure 3. 13 Images of Carl Zeiss Supra 35 VP field-emission SEM and TESCAN VEGA 3 tungsten thermionic emission SEM …........................................... 70

Figure 3. 14 Image of SPI-module sputter coater ........................................ 71

Figure 3. 15 Image of Boss LS1630 laser cutting system............................... 72

Figure 3. 16 Image of RDWorks software graphic interface with two layers ....... 73

Figure 3. 17 Image of Thermo Scientific 1300 Series A2 Laminar-Flow Hood ... 74

Figure 3. 18 Image of VWR CryoPro liquid nitrogen tank ............................. 75

Figure 3. 19 Image of Fisher Scientific ISOTEMP 210 Water Bath ................... 75

Figure 3. 20 Image of VWR $\mathrm{CO}_{2}$ incubator.................................................. 76

Figure 3. 21 Image of Beckman Coulter Allegra 6R Centrifuge ….................... 76

Figure 3. 22 Image of Nexcelom Auto T4 Cellometer ...................................... 77

Figure 3. 23 Image of Nikon TE2000-U inverted microscope sysem ................. 78 
Figure 3. 24 Image of Nikon Eclipse Ti inverted microscope with A1R confocal

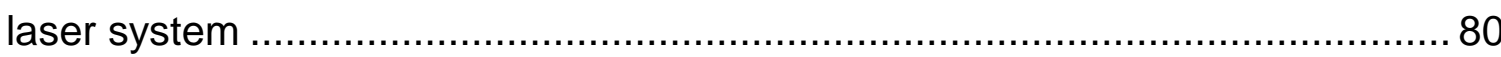

Figure 3. 25 Image of the two walls chambered cover glass system ................. 80

Figure 3. 26 Image of gelatin and PLGA and their composites solution ............. 82

Figure 3. 27 Image of PLA-PEG copolymer synthesis setup ............................ 84

Figure 3. 28 Schematic illustrating (top) and real images (bottom) of the direct-

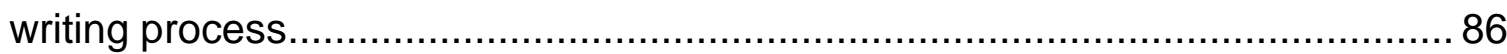

Figure 3. 29 Schematic illustrating and real images of the fabrication of the

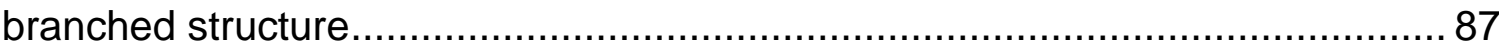

Figure 3. 30 Image of benchtop compression molding .................................. 95

Figure 3. 31 Cells seeded on the scaffold with slow rocking on a nutating mixer in

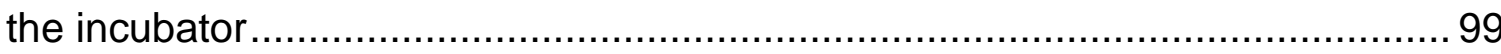

Figure 4. 1 SEM images of gelatin fibers drawn from the solutions ................. 108 Figure 4. 2 SEM images of the biopolymer micro-fibers fabricated with the 3-Axis robotic dispensing robotic system........................................................ 110 Figure 4. 3 SEM images of the gelatin branched structures with different branching angles and branched structures................................................ 112 Figure 4. 4 FT-IR spectra of pristine gelatin, PLGA, and their composites ....... 113 Figure 4. 5 SEM images of representative single suspended micro-fibers ....... 116 Figure 4. 6 SEM images of the representative branched structures from different biopolymers.

Figure 4. 7 SEM images of complex suspend structures fabricated from PLAPEG (75:25) copolymer ... 
Figure 4. 8 FT-IR spectra of pristine PLA, PEG, and PLA-PEG copolymers .... 120 Figure 4. $9{ }^{1} \mathrm{H}-\mathrm{NMR}$ spectra of PLA, PEG, and PLA-PEG copolymer .............. 122

Figure 4. 10 ChemNMR ${ }^{1} \mathrm{H}$ Estimation of the formed copolymer..................... 123

Figure 4. 11 Controllable factors involved in the direct-write process .............. 124

Figure 4. 12 Optical images of the gelatin solution drops with different needles

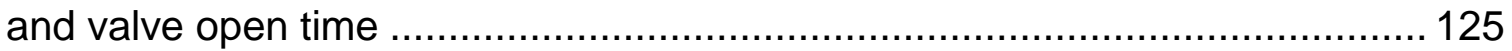

Figure 4. 13 Optical images of direct-write polymer droplet with differing needle/substrate distances across different needle inner diameters 127 Figure 4. 14 Example of measuring the diameter of the micro-fiber from the fibers array by the SEM 128 Figure 4. 15 SEM images of gelatin fibers with different fiber diameters from the DOE. 128

Figure 4. 16 The "main effects" plots of the mean of fiber diameter as a function of concentration, needle ID, feed rate, and fiber length 130

Figure 4. 17 Yield of direct-writing gelatin fibers versus several factors. 131

Figure 4. 18 The viscosity of gelatin solutions under different shear rates 134 Figure 4. 19 The "main effects" plots of the mean of fiber diameter as a function of Processability parameter, Capillary number, and Aspect ratio. 138 Figure 4. 20 Surface plots representing the fiber diameter as a function of Processability parameter, Capillary number, and Aspect ratio 139 Figure 4. 21 Plots of the normal probability and histogram of residuals, as well as residuals against the fitted value and observation order 140 Figure 4. 22 The micro-fiber yield of various direct-written biopolymers 142 
Figure 4. 23 The distribution of the Processability parameters of various biopolymers 145

Figure 4. 24 Comparison of the predicted and the experimental diameters of the direct-write micro-fibers for various biopolymers, composites, and copolymers 146 Figure 4. 25 The degradation profile for various biopolymers, composites, and copolymers 149

Figure 4. 26 Cellular adhesion and viability profiles for various biopolymers.... 151 Figure 4. 27 The comparison of cell density on the bottom of the well plate after applying a BSA coating at different concentrations 152 Figure 4. 28 Fluorescent images of the HDMECs adhered on copolymer films and micro-fibers with different adhesion promoters 153

Figure 4. 29 The quantity of attached cells on the copolymer films and microfibers with treatments of different adhesion promoters 153 Figure 4. 30 Fluorescent images of the HDMECs adhered on the micro-fiber with different seeding density. 155 Figure 4. 31 Confocal images of a single PLA-PEG fiber with the HDMECs cultured and stained to highlight the nuclei (blue) and cytoskeletons (red) ...... 157 Figure 4. 32 Confocal image of a single PLA-PEG fiber with the HDMECs cultured and stained to highlight the nuclei (blue) and VE-cadherin (green) .... 158 Figure 4. 33 SEM image of a single PLA-PEG fiber with the HDMECs cultured for 48 hours 160

Figure 4. 34 Fluorescent images of the HDMECs stained to highlight the live cells (green) and dead cells (red) at different magnification 161 
Figure 4. 35 3D reconstruction confocal image of HDEMCs on PLA-PEG

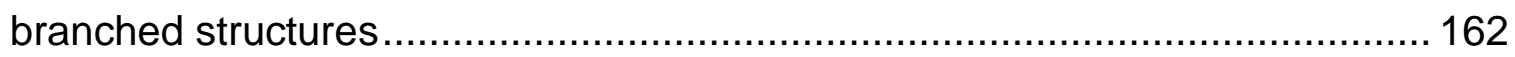

Figure 4. 36 Optical images of the vascularized sheets ............................... 164 Figure 4. 37 Fluorescent images and 3D reconstruction confocal images of the single micro-fiber and branched structure 166 Figure 4. 38 Optical and SEM image of the dehydrated vascularized sheet after radial cutting 168

Figure 4. 39 SEM images of the cross-sectional view of the vascularized sheets after three days and two weeks culture 171 


\section{LIST OF TABLES}

Table 2. 1 Summary and comparison of blood vessel anatomy ....................... 10

Table 2. 2 List of common commercially-available endothelial cells................ 14

Table 2. 3 Properties of common natural and synthetic biopolymers ................ 18

Table 2. 4 A general comparison of natural and synthetic biopolymers in the

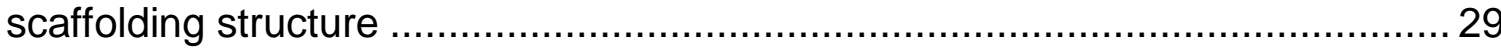

Table 2. 5 Comparison of different biopolymer fabrication techniques .............. 48

Table 2. 6 Summary of recent studies on microvascular structures using the

different fiber fabrication techniques and cell types ......................................50

Table 3. 1 The operation parameters for cell culture fiber frame with a recessed

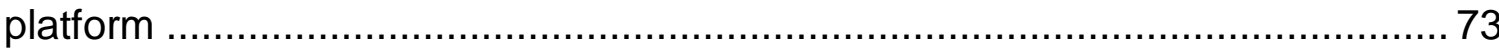

Table 3. 2 Copolymer synthesis component mass ratio .................................. 84

Table 3. 3 Selected factors and their levels used in the design of experiments . 90

Table 3. 4 Biopolymers used to validate the gelatin-based empirical model ...... 92

Table 3. 5 Collagen Gel Solution Components............................................ 103

Table 4. 1 Solvent and concentration trials for gelatin ................................ 107

Table 4. 2 TFE solution concentration trials for PLGA and gelatin/PLGA

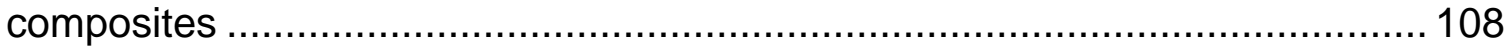

Table 4. 3 Solvent trials for PLA and PLA-PEG copolymers ......................... 114

Table 4. 4 Selected concentration of PLA and PLA-PEG copolymer................ 115 
Table 4. 5 Gelatin polymer drop diameter measurements ............................. 126

Table 4. 6 The ANOVA table for fiber diameters …..................................... 129

Table 4. 7 Physical properties of the gelatin solutions .................................. 132

Table 4. 8 Factors, symbols, and dimensions of the response, process factors,

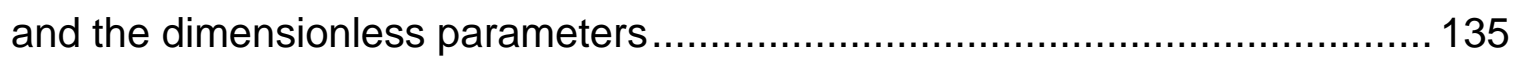

Table 4. 9 Calculated dimensionless parameters of the gelatin solutions ........ 135

Table 4. 10 The ANOVA for the transformed response of the empirical model 137

Table 4. 11 Physical properties of various biopolymers solutions.................... 143

Table 4. 12 Calculated dimensionless parameters of biopolymer solutions .... 144

Table 4. 13 Measurement of the prediction error .......................................... 147 


\section{CHAPTER 1: INTRODUCTION}

Tissue and organ failure caused by disease or injury has become a major health dilemma in the world, accounting for $\sim 40 \%$ of the annual total causes of death in 2015 [1]. There are four principal therapeutic strategies for treating tissue and organ failure in patients: surgical repair, artificial prostheses, mechanical devices, and transplantation (human or xenotransplantation) [2]. Although these therapies have saved innumerable patients' lives, they are still far from perfect solutions. A surgical repair usually leads to long-term complications for the patient. For example, hepatic resection often develops serious postoperative morbidity, the most common being bile leak and associated perihepatic abscess [3]. Artificial prostheses and mechanical devices neither perform the full physiological function nor adequately repair/restore full organ function. Moreover, artificial prostheses and mechanical devices are generally subjected to wear upon long-term implantation, which can induce an inflammatory response in the patient [4]. For some patients with end-stage organ failure, such as heart and liver failure, transplantation is the most effective way to save their lives. However, organ transplantation suffers from severe donor shortage. Specifically, according to Organ Procurement and Transplantation Network Data Reports, there are $116,000+$ patients on the national transplant waiting list as of August 2017. Only 33,611 transplants were performed in 2016, and the vast imbalance between the 
number of organ donors and patients waiting for donor transplants worsens every year [5].

Tissue engineering is an approach with significant potential for solving the organ donor shortage problem. The concept of tissue engineering was first introduced by Robert Langer and Joseph Vacanti in the first NSF-sponsored meeting on tissue engineering in 1988, which described the concept as "attaching cell preparations to bioerodable artificial polymers in cell culture and then implanting this polymer-cell scaffold into animals" [6]. In 1993, the pioneering paper entitled "Tissue Engineering" appeared in Science, which further defined tissue engineering as "an interdisciplinary field that applies the principles of engineering and the life sciences toward the development of biological substitutes that restore, maintain, or improve tissue function" [7]. The main goal of tissue engineering is to create functional tissues and organs in vitro and then transplant them into the host. After decades of rapid development, scientists and engineers have begun to design and engineer vital organs/structures in the laboratory, including the liver, spinal cord, blood vessels, cartilages, hearts valves, skin, bones, intestines, urological structures, tendons, and muscles [8-12]. Overall, most engineered tissues have not realized commercial success, primarily due to the need for a sustainable, highly branched system of blood vessels and microvascular network to enable the exchange of nutrients and waste products. Only a few engineered tissues, such as skin and cartilage [13-18] have been successful clinically since they can be manufactured without vascular or neural networks. However, progress on producing larger and more complex tissues and organs has hampered by the 
lack of ability to create engineering microvascular network that meet the metabolic needs of the tissues after implantation [19-21].

Thus, the capability to selectively produce a microvascular network has become an emerging field of tissue engineering. Both in vivo and in vitro methods have been proposed for the development of the engineered microvasculature. Vasculogenesis and angiogenesis are the two fundamental processes involved in new blood vessel formation in vivo $[22,23]$. There are three different models that have been studied in vivo to form microvascular lumen: (1) vacuole formation and coalescence, (2) wrapping around extracellular space, and (3) cell death and phagocytosis [24]. This research may answer many important questions about microvasculature development, such as how endothelial cells could form a luminal or apical plasma membrane and how existing plasma membranes could rearrange to form a vascular lumen. However, in vivo studies are time-consuming and highcost. Also, the biology of animals, mostly mice[25], is different from humans and the rejection reaction could be a severe problem.

Due to these complications, several methods that have been developed to generate microvasculature in vitro. The idea of prevascularization was first reported by Mikos et al. in 1993 [26], with the main concept being to incorporate endothelial cells into decellularized tissue or an ECM [27-30]. However, the network patterns of microvasculature were usually formed randomly and the diameters of the produced vessels were uncontrollable. Another strategy to generate blood vessels in vitro is to create lumen structures via microfluidics or bio-ink three-dimensional printing techniques[31-33]. Although a microvascular 
network with desired design pattern could be formed, most of these techniques suffered from an unrealistic minimum lumen diameter (several hundred microns) or non-cylindrical shapes that are dissimilar to natural forming microvasculature in the body.

With the advent of biopolymers, a fiber scaffold could be used to support and guide endothelial cells to generate a microvascular network in vitro. Currently, traditional techniques such as dry spinning, wet spinning, and electrospinning are employed to produce a variety of fibrous structures including suspended microfibers, fibrous monoliths, porous films, and nano-fibrous mats. However, the limitations on the accurate placement of fibers to predefined locations with controlled diameters have impeded the further advancement of these types of microvascular networks. Thus, developing a three-dimensional microvascular scaffold with a specific pattern and biomimetic diameter to provide temporary mechanical support and stimulate endothelial cell adhesion, growth and morphogenesis into a vessel would be beneficial. Our research group has developed a "Direct-write" method using a 3-Axis robotic dispensing system to fabricate suspended micron/sub-micron polymer fibers. The driving mechanism behind this process harnesses the surface tension of liquid bridges to promote the controlled thinning of a macroscale polymer solution filament into the desired microscale fibers. The advantage of this process is the ease of obtaining arrays of precisely-positioned fibers with controllable diameters in three-dimensional space. Several scaffold design criteria should be met: (1) The scaffold size and spatial position should be controlled within the microvascular scale. (2) The scaffold must 
be capable of adhering and proliferating the endothelial cells. (3) The scaffold must be robust enough to remain during cell proliferation so as to allow a monolayer of endothelial cells to be formed and supported by ECM. (4) The scaffold should degrade in a reasonable time to develop the final capillary network.

\subsection{Purpose of the Study}

The overall goal of this project was to develop a model for the construction of a precisely positioned, three-dimensional, suspended biopolymer scaffold with controlled diameters and a proof-of-concept of a physiological microvascular networks in vitro. The studies presented in this work focus on: (1) direct-writing the three-dimensional oriented biopolymer scaffold with microvascular-scale diameters by using a 3-Axis robotic dispensing system; (2) developing and validating an empirical model of the direct-write process based on a design of experiment, characterization, and dimensional analysis; (3) determining a recipe for seeding the Human Dermal Microvascular Endothelial Cells (HDMECs) on the fabricated scaffold to form a confluent HDMECs monolayer; and (4) embedding the HDMECs-covered scaffold into the collagen hyrogel to form a vascularized sheet in order to demonstrate the conceptual "modular tissue", which could be potentially assembled to bulk tissue.

\subsection{Specific Aims}

The specific aims for this project are: 
Specific Aim 1: To fabricate precisely-positioned, suspended micro-fibers and branch structures with microvascular-scale diameters. These micro-fibers and branch structures will be fabricated from a variety of biodegradable polymers via the direct-write technique.

Specific Aim 2: To generate and validate an empirical dimensionless model of the direct-write process. Gelatin will be used to perform a design of experiment and characterization for the empirical dimensionless model generation. Poly(Lactic-co-glycolic acid) (PLGA), gelatin/PLGA composites, Polylactic acid (PLA), and Polylactic acid- Polyethylene glycol (PLA-PEG) copolymer will be used to validate the empirical dimensionless model.

Specific Aim 3: To seed the endothelial cells on the selected scaffold by following the scaffold-covering strategy. HDMECs will be used to grow a confluent monolayer on the surface of the scaffold. The monolayer should spread along the axis and around the circumference of the fiber.

Specific Aim 4: To encapsulate the cell-covered scaffold into ECM and continue to culture to obtain the vascularized sheet. Type I collagen from rat tail will be used as the ECM. The scaffold degradation and Cell-Scaffold-ECM interaction will be evaluated through various imaging techniques.

\subsection{Significance of the Study}

This project aims to generate a conceptual solution for creating a threedimensional microvascular network in vitro. In this project, a biodegradable 
microvascular scaffold will be developed and fabricated by integrating direct-write technique through a 3-axis robotic dispensing system. The fabrication will be characterized and an empirical model will be generated to predict the scaffold diameters. Instead of using human umbilical vein endothelial cells (HUVECs) in the most reported prevascularization research, Human Dermal Microvascular Endothelial Cells (HDMECs) will be chosen because they have a real common origin with human blood and lymphatic capillaries. HDMECs will be seeded on the scaffold, and a confluent monolayer will be formed on the surface of the scaffold. ECM will be added to support the endothelial cells and form a cell-viable, flexible, and free-standing vascularized sheet, which could be potentially assembled to a three-dimensional microvascular network. To date, the combination of using the direct-write technique to fabricate biopolymer scaffold and create a microvascular network by scaffold-covering strategy has not been reported. 


\section{CHAPTER 2: BACKGROUND}

2.1 Overview of the Microcirculation System

Microcirculation is one of the most essential components in the human circulation system, and $84 \%$ of systemic circulation occurs here. The primary function of the microcirculation system is to transport nutrients to the tissues and removal of cell excreta[34, 35]. The microcirculation system is ubiquitously distributed in our bodies, and the peripheral circulation has about 10 billion capillaries. The estimated surface area of these capillaries may cover $1 / 8$ of a standard US football field (500 to $\left.700 \mathrm{~m}^{2}\right)$. Most of the functional live cells of the body are in about 20 to 30 micrometers away from a capillary[34]. Cells too distant ( $>200 \mu \mathrm{m})$ from capillary would not survive and proliferate due to the lack of the supply of oxygen and nutrients[36].

\subsubsection{Structure of the Capillary System}

In general, the capillary system consists of three primary components: 1) Arterioles - artery will form 6 to 8 branches after entering an organ and become smaller, which generally have internal diameters of 20 to 40 micrometers. 2) Capillary - arterioles further branch 2 to 5 times, leading to only 5 to 10 micrometers at their ends. 3) Venules - capillaries converge together to form small vessels before exiting an organ and connecting to the vein. The venules are usually smaller than arterioles in diameter. The typical arrangement of the capillary bed is shown in Figure 2.1. The arterioles have a robust muscular coat, and they 
are expandable and contractile thus they can control the blood flow into each tissue. The terminal arterioles, also known as metarterioles are encircled by smooth muscle fibers at the intermittent points. The muscular coat is not continuous in metarterioles. The capillaries also encompassed by smooth muscle fibers just like metarterioles. This configuration is defined as precapillary sphincter, and it can control the entrance of the capillary. Although the venules are not very muscular like arterioles, they can still expense and contract due to lower pressure in venules[34, 35].

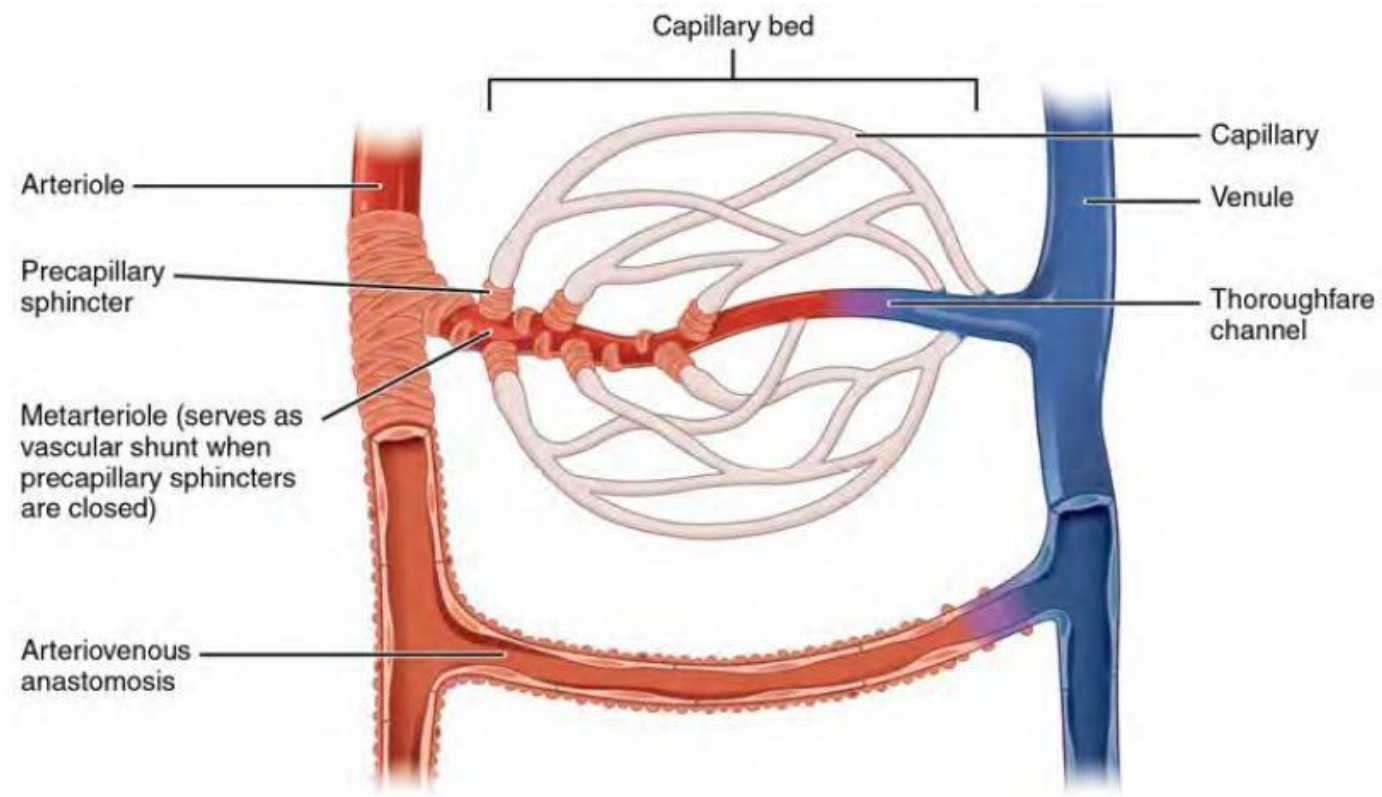

Figure 2. 1 Illustration of the structure of the capillary bed. Source:[37]

\subsubsection{Structure of the Vessel Wall}

Arterioles are the smallest arteries, and their structures are often simple. Arterioles have a poorly-defined tunica externa. In some larger arterioles, the 
tunica media is consists of one or two layers smooth muscles cells with thickness $\sim 20 \mu \mathrm{m}[35]$. In contrast, the tunica of the smaller arterioles has scattered smooth muscle cells with incomplete cell layer. Venules have similar structures as arterioles, generally with no elastic tissue and less smooth muscles and fibrous tissue. The capillary wall is constructed of single-layer endothelial cells, and the endothelial cells are surrounded by a basement membrane on the outside. Capillaries are extremely thin ( 0.5 micrometers $)$ and permeable, thus the nutrients and wastes can be exchanged between circulation systems and the cells[34]. The summary and comparison of these three vessels in microcirculation is shown in Table 2.1[38].

Table 2. 1 Summary and comparison of blood vessel anatomy. Source:[38]

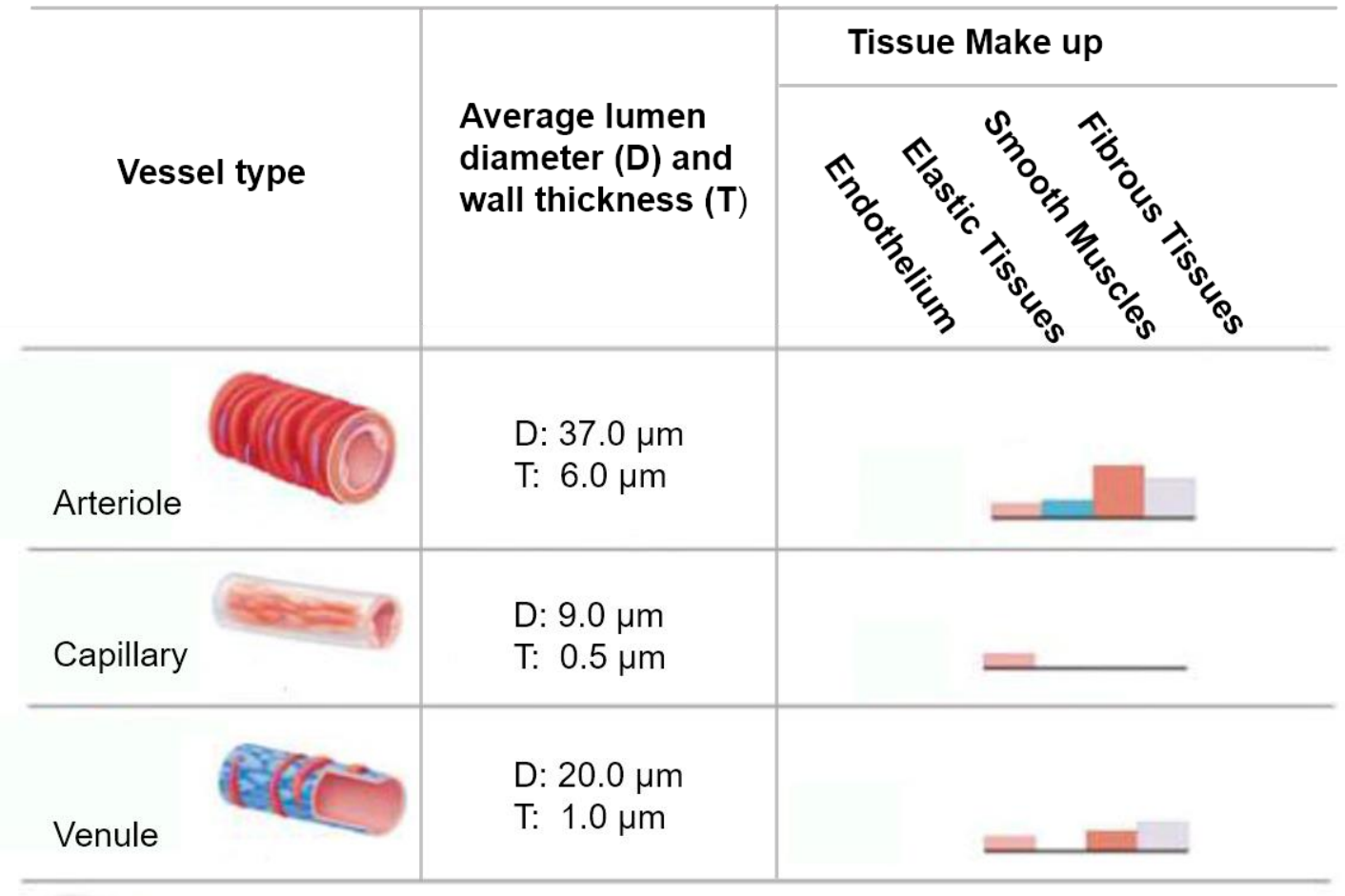




\subsubsection{Structure of the Capillary Wall}

As now we know endothelial cell layer exists in all the microcirculation vessels, a further study of the capillary wall could help us to understand the structure and mechanism for the tissue engineering better. Figure 2.2 is an illustration of the structure of the capillary wall. Small "slit pores" with average width about 6 to 7 nanometers could be found between two adjacent endothelial cells, known as intercellular cleft. The cleft connects endothelial cells together by a tiny protein bridge, and the thin slit could make sure fluid can freely flow through it. The many small caves in endothelial cells are plasmalemmal vesicles. The real functions of these caves are still not clear, and some studies show they involve transporting nutrients molecules across endothelial cells' membrane[34].

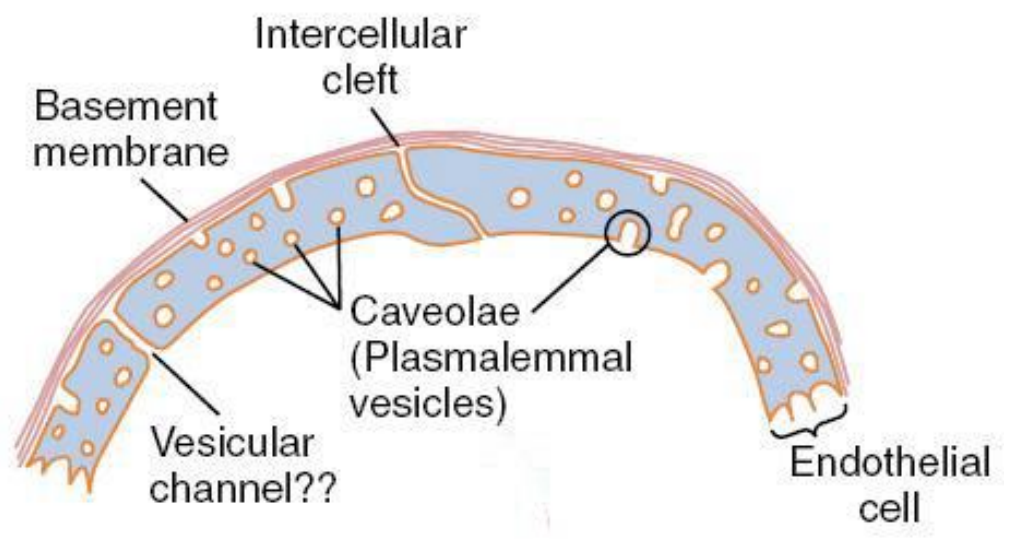

Figure 2. 2 Illustration of the structure of the capillary wall. Source: [34]

In general, there are three types of capillaries according to the degree of permeability caused by different structures: 1) Continuous Capillaries - Most common capillaries that could found in muscle and skin. The intercellular clefts are 
very small to ensure the least permeability. 2) Fenestrated capillaries - Often found in kidneys and small intestines where a high rate of exchange is needed. The relatively large pores make sure small molecules could pass through the membrane. 3) Sinusoidal capillary - Specifically found in liver, bone marrow, and spleen which are usually loose structures. The intercellular clefts are wide enough to pass through the entire cells. All three types of capillaries are shown in Figure 2.3. The blood-brain barrier is an exception that the endothelial cells have no intercellular clefts and tight junctions are encircled by the whole capillary. Only a few selected vital molecules could pass through the membrane.
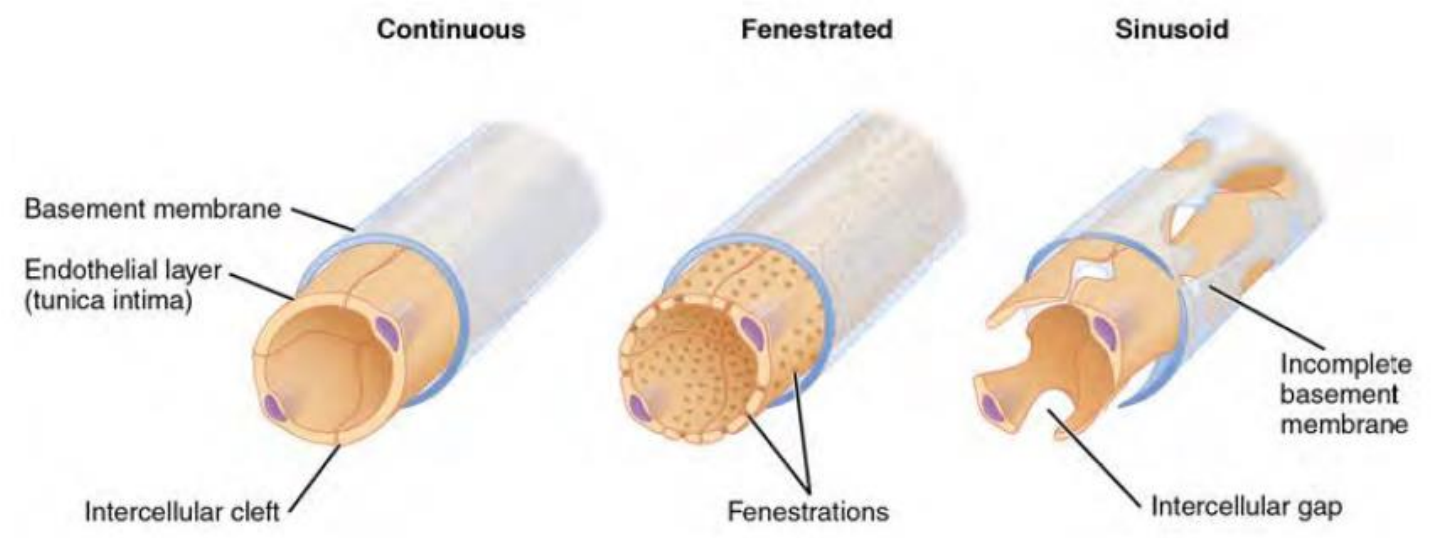

Figure 2. 3 Illustration of three structures of capillaries: continuous capillary, fenestrated capillary, and sinusoid capillary. Source: [37]

\subsubsection{Function of the Capillary System}

Instead of flowing continuously in the artery and vein, blood flows intermittently in the capillary system. The intermittent contraction of metarterioles and precapillary sphincters control the capillaries open and shut every few seconds 
or minutes. The most important function of the capillary system is to exchange water, nutrients, and other substances between the blood and interstitial fluid, and the most efficient way to exchange is diffusion. Figure 2.4 is showing blood flows through the capillary's lumen, and countless water molecules and dissolved nutrients diffuse in and out through the capillary wall. Different molecules may have different diffusion paths because of the molecules' sizes, properties, and concentrations. Such as lipid-soluble molecules directly diffuse through the capillary wall, whereas water-soluble substances diffuse through the holes of the intercellular "pores" in the capillary's membrane.

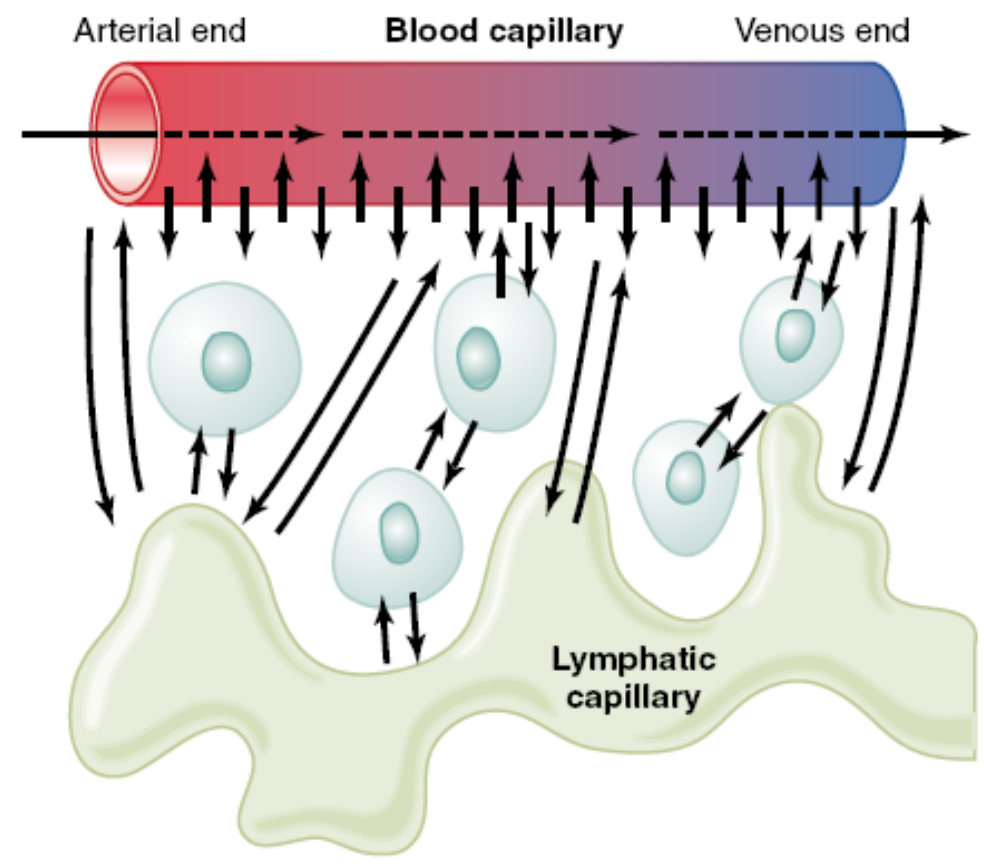

Figure 2. 4 Diffusion of fluid molecules between the capillary and interstitial fluid space. Source: [34] 


\subsubsection{Endothelial Cell}

As we discussed before, the endothelial cells are the most fundamental component of the microcirculation system. They differ in structures and functions according to their ubiquitousness in different vascular locations and microenvironments[39, 40]. We could briefly classify endothelial cells into two categories: (1) Large vessels endothelial cells, and (2) Microvascular endothelial cells. Microvascular endothelial cells will be discussed in this work since we are trying to engineer the capillary network. One significant property of the microvascular endothelial cell is that they are highly active and intimately involved in numerous physiological processes. The list of the common commercially available endothelial cell is shown in Table 2.2.

Table 2. 2 List of common commercially-available endothelial cells. Source:[41]

\begin{tabular}{|c|c|c|}
\hline Cell type & Common source & Examples \\
\hline $\begin{array}{l}\text { Large } \\
\text { vessels } \\
\text { endothelial } \\
\text { cells }\end{array}$ & $\begin{array}{l}\text { Umbilical vein and } \\
\text { artery, the aorta, the } \\
\text { coronary artery, and } \\
\text { the pulmonary artery }\end{array}$ & $\begin{array}{l}\text { Human Umbilical Vein Endothelial Cells } \\
\text { (HUVEC), } \\
\text { Human Umbilical Artery Endothelial Cells } \\
\text { (HUAEC), } \\
\text { Human Aortic Endothelial Cells (HAoEC), } \\
\text { Human Coronary Artery Endothelial Cells } \\
\text { (HCAEC) } \\
\text { Human Pulmonary Artery Endothelial Cells } \\
\text { (HPAEC) }\end{array}$ \\
\hline
\end{tabular}




\begin{tabular}{l|l|l}
\hline & & Human Dermal Microvascular (HDMEC), \\
Microvascular & Dermal, lung, & Human Cardiac Microvascular Endothelial \\
endothelial & Cells (HCMEC), \\
cells & tissues & Human Pulmonary Microvascular \\
& & Endothelial Cells (HPMEC), \\
& & Human Uterine Microvascular Endothelial \\
& & Cells (HUtMEC) \\
\end{tabular}

\subsection{Extracellular Matrix}

Extracellular matrix (ECM) are ubiquitous noncellular components that could be found in almost all the tissues and organs. The ECM not only acts as a three-dimensional structural scaffold for cells, but also functions as an adhesive substrate, presents, sequesters, and stores growth factors, senses and transduces mechanical signal, and serves as signals for morphogenesis and differentiation[42, 43]. Fundamentally, the ECM is composed of water and various macromolecules. Those macromolecules have been classified into two main groups by composition: Proteoglycans and Fibrous proteins [44, 45]. More than 30 different proteoglycans are known in humans, most of which are composed of glycosaminoglycans (unbranched polysaccharide chains) that are covalently linked to a specific core protein. According to their core protein, the proteoglycans could be classified as three groups: Modular proteoglycans, Small leucine-rich proteoglycans (SLRPs), and Cell-surface proteoglycans (Figure 2.5) [46]. The formed glycosaminoglycans hydrogel fill the majority of the extracellular interstitial space. Recently studies 
suggest that proteoglycans play a prominent role in a wide variety of functions, such as buffering, hydration, signaling, resisting compressive force, and binding growth factors $[45,47,48]$.

Fibrous proteins could be classified as collagens, fibronectins, elastins, and laminins(Figure 2.5) $[45,49]$. Among those, collagens are the most common fibrous proteins found in the ECM, and more than 28 different types of collagen have been identified in the human body so far. Collagen could provide tensile strength, regulate cell adhesion, and direct tissue development[43]. A typical collagen molecule has a complex hierarchical structure that contains a signature triple-helix structure (300 $\mathrm{nm}$ in length and $1.5 \mathrm{~nm}$ in diameter). The collagen molecules could form fibrils and networks depending on the types of collagen. Type I, II, III, V, and XI could self-assemble into fibrils that can resist shear, tensile, and pressure force, whereas type IV, VIII, and X could form networks which are incorporated into the basement membrane[47].

Fibronectin is another important non-collagenous fibrous protein. The fibronectin molecules are usually composed of multiple chains, each encoded by single genes. Fibronectin could be stretched many folds over its resting length by cellular traction force, and it could also direct the organization of the interstitial ECM[45, 50]. In addition, the adhesive Arg-Gly-Asp (RGD) sequence could be found in the hydrophilic loop of fibronectin. Thus, fibronectin could also act as an adhesive substrate for cell and other matrix proteins due to the fact that RGD sequences are critical for recognition and binding to many integrins [43]. 
Recently studies suggest that ECM play an essential role in both angiogenesis and vasculogenesis. During the blood vessel generation process, the endothelial cells migrate, proliferate, and eventually form the lumen structure in the ECM[51]. Researchers have proved that integrin receptors of the ECM play a critical role in regulating the cell shape through the change in the cytoskeleton and forming the lumen shape via the difference in the cell-cell interactions[51, 52]. Several in-vitro studies have demonstrated that the endothelial cells could attach to both collagen Type I hydrogels and basement membrane Matrigel ${ }^{\mathrm{TM}}$ and subsequently migrate and align to generate capillary-like network[53, 54].

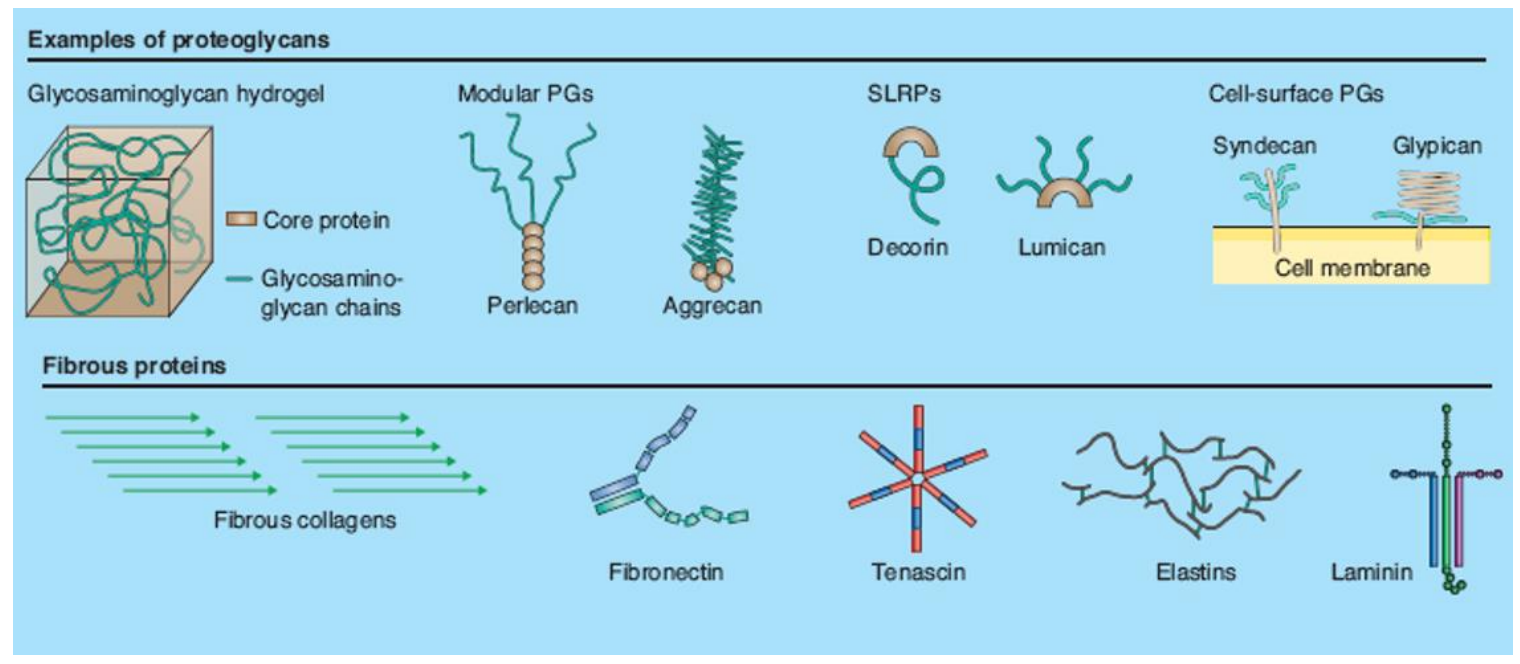

Figure 2. 5 Examples of common proteoglycans and fibrous proteins. Source: [45]

\subsection{Biopolymers}

Typically, biomaterials can be classified into three groups: ceramics, metals, and biopolymers[55]. Ceramic and metal scaffolds are predominantly used in orthopedic applications and thus will not be covered in this dissertation. However, 
biopolymers have been implemented extensively as microvascular scaffold materials due to their excellent biocompatibility, biodegradability, and ease of processing characteristics. Based on the chemical structure and origin, biopolymers can be further divided into three groups: (1) Natural biopolymers, (2) Synthetic biopolymers, and (3) Composites. When considering which type of biopolymer to use, there are several chemical and physical properties that must be considered, beyond the typical biocompatibility and toxicity of the material, such as the material's mechanical properties, biodegradability, and processability (Table 2.3 ), in addition to its ability to promote cell adherence and proliferation.

Table 2. 3 Properties of common natural and synthetic biopolymers. Source: [5659]

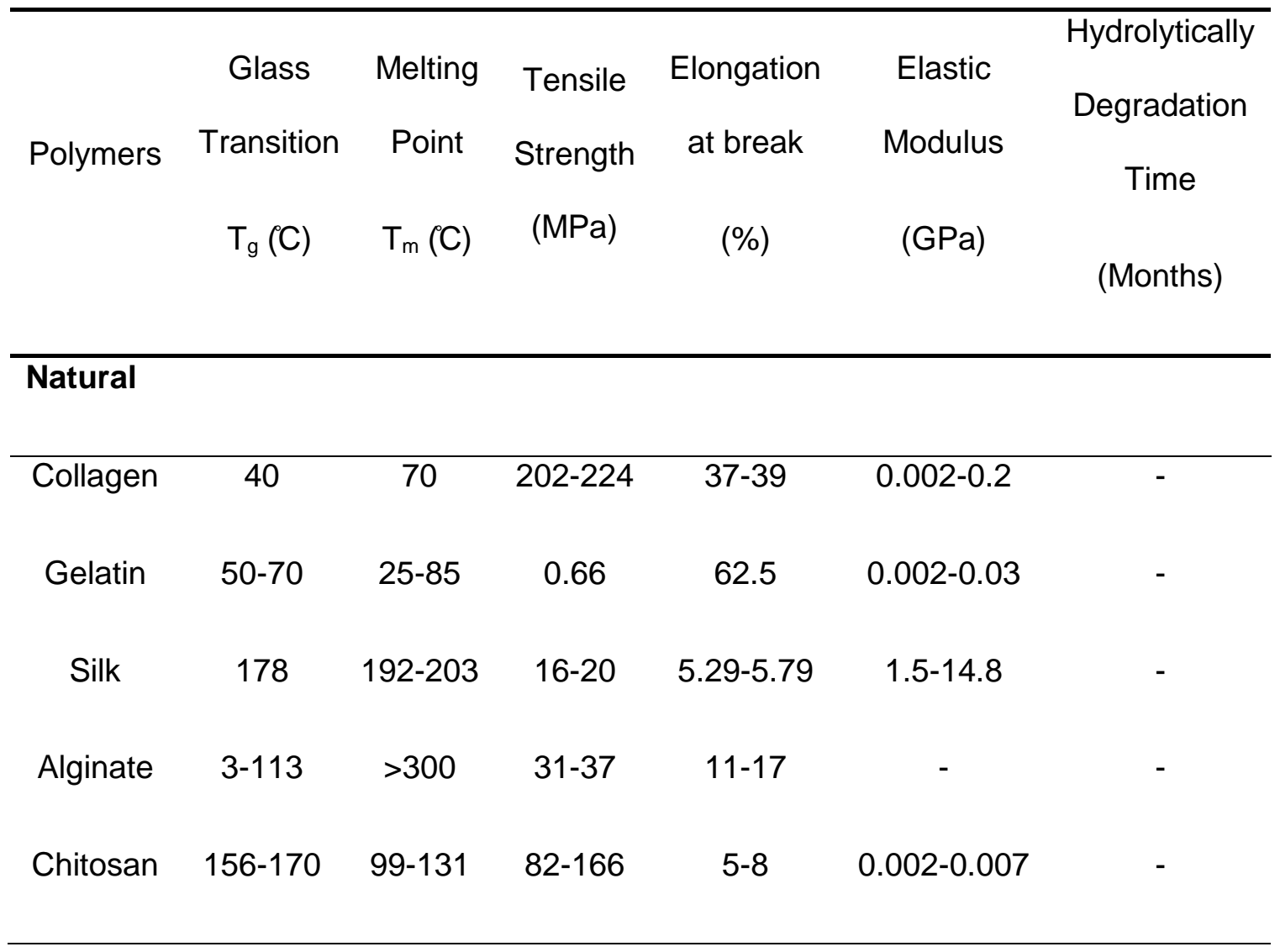




\section{Synthetic}

\begin{tabular}{|c|c|c|c|c|c|c|}
\hline PGA & $35-40$ & $225-230$ & $60-99.7$ & $1.5-20$ & $6-7$ & $6-12$ \\
\hline PLLA & $40-70$ & $130-180$ & $15-150$ & $3-10$ & $2.7-4.14$ & $24-60$ \\
\hline PDLLA & $55-60$ & - & $27.6-50$ & $2-10$ & $1-3.45$ & $\sim 12$ \\
\hline PLGA & $45-55$ & - & $41-55.2$ & $2-10$ & $1-4.34$ & $1-6$ \\
\hline PCL & $-65-60$ & $58-63$ & $20.7-40$ & $300-1000$ & $0.21-0.44$ & $>24$ \\
\hline
\end{tabular}

\subsubsection{Natural Biopolymers}

Natural biopolymers are usually produced from plants or animals, and can even be derived from microbial systems. Natural biopolymers have inherent bioactivity present such as receptor binding ligands for cells. For example, Fittkau et al. demonstrated the ability of biomimetic peptides, such as RGD, to selectively affect adhesion and migration of human microvascular endothelial cells on polyethylene glycol (PEG) surfaces[60]. In addition, materials from naturally derived origins increase the potential for the biopolymers to have similar chemical and/or physical characteristics as ECM components, which, in turn, may not induce a chronic inflammatory or immunological response or be toxic to the cells, which is often found in synthetic biopolymers[61]. Natural biopolymers can be recognized by the biological environment and channeled into metabolic degradation by undergoing enzymatic degradation through cleavage of the enzyme-sensitive 
bonds, and eventually lead to erosion of the biopolymer[62]. In specific applications using a sacrificial scaffold, the degradation rate of the natural biopolymers can be accelerated by increasing the concentration of the enzymes[63]. Conversely, the degradation rate can be reduced by cross-linking[64] the biopolymer in order to avoid rapid degradation of the scaffold which could lead to the collapse of the developing microvascular network. Notwithstanding, natural biopolymers also present some disadvantages, such as slow processing and inherent batch-tobatch variations[65].

Natural biopolymers can be divided into four groups: (1) Polysaccharides; (2) Proteins; (3) Nucleic Acids; and, (4) Viruses (Figure 2.6) [66]. Nucleic Acids and viruses are rarely used in microvascular scaffold applications. However, proteins such as collagen, fibrin and silk fibroin, and polysaccharides such as chitosan and alginate are commonly used in vascular/microvascular development applications.

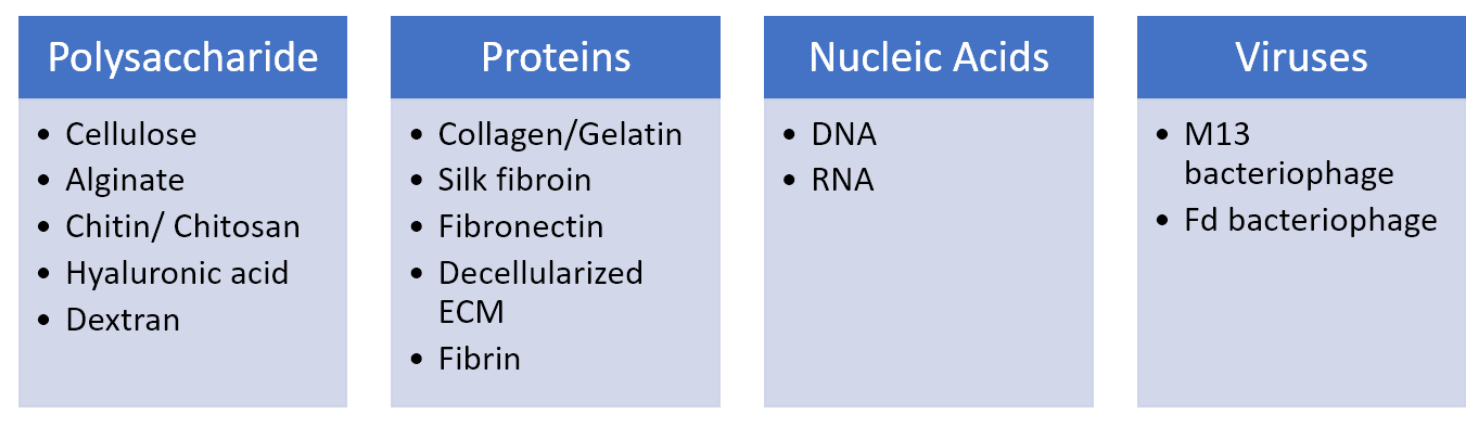

Figure 2. 6 Classification of several common natural biopolymers 


\subsubsection{Collagen/Gelatin}

As it has been introduced in the previous ECM section, collagen is the most abundant protein in mammals, and it is the major component of connective tissues, skin, bone, cartilage, and tendons. On the other hand, gelatin is a biomacromolecule derived through the partial hydrolysis of collagen, which converts the triple-helix structure into a coil structure due to cleavage of the hydrogen and covalent bonds (Figure 2.7). As a result, gelatin has similar biocompatibility and biodegradability characteristics to collagen, but collagen is water soluble.

Collagen and gelatin have both been extensively used in tissue engineering because of their biocompatibility, weak antigenicity, high mechanical strength, and tunable biodegradability by controlling the degree of cross-linking[67]. Collagen and gelatin are commonly used as hydrogels in tissue engineering $[68,69]$. They can also be processed into a variety of forms such as fleeces, sheets, tubes, fibers, powders, and injectable solutions[70]. Moreover, these two kinds of protein are popular natural biopolymers that are widely used to produce fibrous meshes and scaffolds using electrospinning techniques [71-73]. 


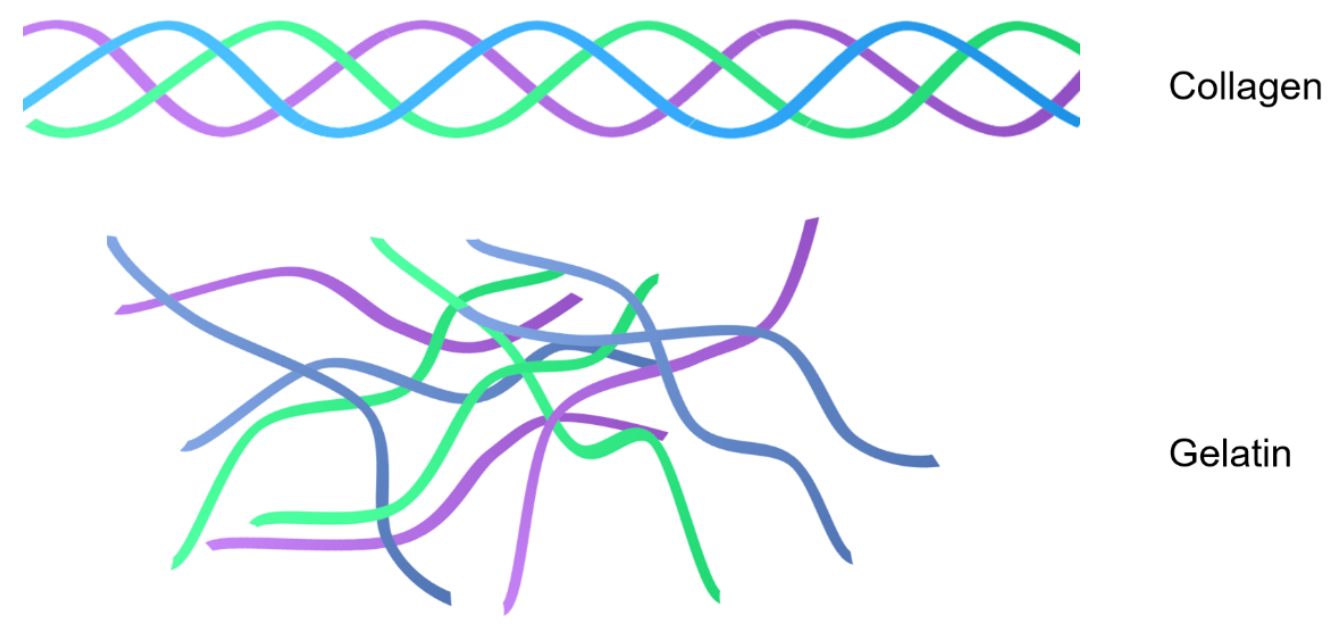

Figure 2. 7 Collagen triple helical molecules structure and gelatin coil structure. Source: [74]

\subsubsection{Silk fibroin}

Silk fibroin is a natural protein commonly produced by arthropods such as silkworms and spiders[86]. It exhibits a unique and useful combination of properties such as good biocompatibility, non-inflammatory, excellent mechanical strength, and low degradation rate. The silk fibroin molecule structure mainly consists of glycine, alanine, and serine. In general, scaffolds made from silk fibroin exhibit lower cell infiltration compared to other biopolymers. As a result, a number of techniques have been employed to improve cell attachment to the scaffold surface, including plasma treatment, cross-linking of the cell-binding domain, and even genetic engineering approaches [58]. Silk fibroin has been processed into films, nanofibers, membranes, mats, nets, hydrogels, and porous sponges using a variety of methods such as wet spinning, electrospinning, and microfluidic spinning[70, 87-89]. 


\subsubsection{Chitin/Chitosan}

Chitin commonly comes from the exoskeleton of arthropods and insects or the cell walls of fungi. Chitosan is a deacetylated derivative of chitin and a polysaccharide. Although they are both semi-crystalline biopolymers with good biocompatibility and biodegradability, chitosan attracts more attention because it is soluble in aqueous acidic media. Chitosan can easily interact with adhesion proteins, growth factors and other proteins since it has a linear monomeric bond[75]. The degradation of chitosan depends on its molecular weight, the degree of deacetylation, and the residual amount of acetyl content. Chemical modification, such as cross-linking, can be easily performed due to chitosan containing hydroxyl and amino moiety functional groups[76]. Wet spinning, electrospinning, microfluidic spinning, and solvent casting have been reported as processing techniques for fabricating chitin/chitosan into gels, films, particles, membranes, fibers, and scaffolds for a large number of different applications[77-80].

\subsubsection{Alginate}

In addition to chitosan, alginate is another extensively studied natural polysaccharide that is typically extracted from brown algae (Phaeophyceae) by aqueous alkali solution treatment. Alginate is composed of guluronic acid (GBlocks) and mannuronic acid (M-Blocks)[81]. Different sources and production processes usually yield different lengths and sequential distribution of these blocks; thereby, directly affecting the molecular weight of alginate. The molecular weight can influence the degradation rate and mechanical properties of alginate-based biopolymers, for example, higher molecular weight alginate has a slower 
degradation rate[82]. As a U.S. Food Drug Administration (FDA) approved polymer, alginate has many applications in the field of biomedicine due to its excellent biocompatibility, biodegradability, and low production cost. Alginate is highly suitable for hydrogels because it is hydrophilic, water-soluble and thickens in neutral conditions. Alginate has been widely used to encapsulate various cells and growth factors to fabricate cell-laden structures because it can be rapidly solidified in calcium chloride $\left(\mathrm{CaCl}_{2}\right)$ solution[83, 84]. In addition, alginate can be processed into a fiber or fibrous structures by microfluidic spinning and other techniques [8589].

\subsubsection{Synthetic Biopolymers}

The first resorbable synthetic biopolymer commercially produced was poly(glycolic acid) (PGA), which was developed by American Cyanamid Co. in 1962 and commercialized in 1970. Since that time, a number of other resorbable synthetic biopolymers have been manufactured, including but not limited to poly(lactic acid) (PLA), poly(lactide-co-glycolide) (PLGA), poly(caprolactone) (PCL), etc. In synthetic biopolymer design and manufacture, the material's properties such as mechanical properties, hydrophilicity, and even bioactivity can be customized by either chemical methods, such cross-linking with functional groups, or physical methods, such as copolymerization with specific monomeric units. The degradation rate can also be tuned by adjusting the monomer's concentration for drug delivery and tissue engineering[90]. For example, poly (Lactide-co-Glycolide) (PLGA) is well known for shorter degradation times at 
higher glycolic acid concentrations. Most of the synthetic biopolymers undergo hydrolytic degradation since they are usually associated with hydrolytically labile chemical bonds such as esters, orthoesters, anhydrides, carbonates, amides, urethanes, ureas, etc.[91]. The hydrolysis has its own downside such as the byproduct carbon dioxide, which can lower the local $\mathrm{PH}$ and result in cell and tissue necrosis. Another disadvantage of some synthetic biopolymers is they can be biologically inert and may not promote cell adhesion and proliferation when used as a scaffold. The cellular interaction with synthetic biopolymer scaffolds have been shown to be improved by both physical and chemical methods, including plasma treatment, covalent tethering of functional groups and augmentation with bioactive molecules, such as gelatin and fibronectin[92, 93].

Synthetic biopolymers can be roughly divided into aliphatic polyesters, poly(amino acids), polycarbonates, and others based on their chemical structures (Figure 2.8). Among them, aliphatic polyesters such as poly(lactic acid) (PLA), poly (Lactide-co-Glycolide) (PLGA), and poly( $\varepsilon$-caprolactone) (PCL) have been extensively investigated for vascular/microvascular applications.
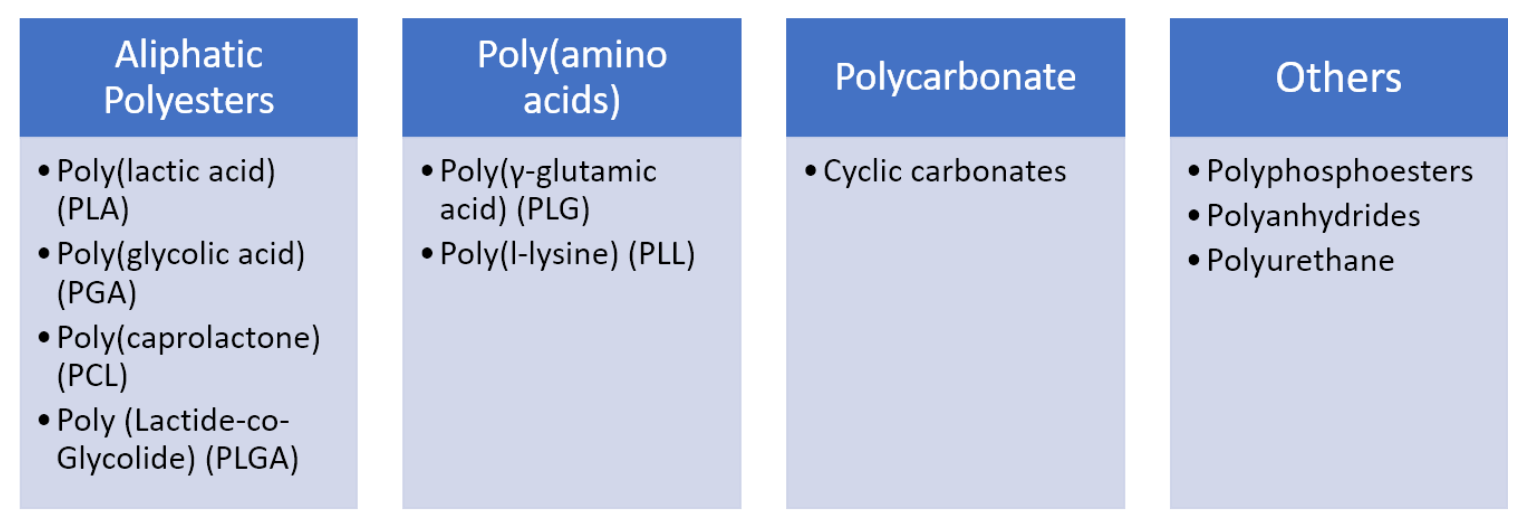

Figure 2. 8 Classification of several common synthetic biopolymers 


\subsubsection{Poly(lactic acid)}

Poly(lactic acid) (PLA) is a hydrophobic thermoplastic polymer. The monomer lactic acid can be derived from an abundant number of natural, renewable feedstock such as starch, wood chips or sugarcane[94]. Lactic acid has two stereoisomerism forms: L-Lactide and D-Lactide. The polymerization of these monomers leads to three morphologically distinct polymers namely, poly(L-PLA) (PLLA), poly(D-PLA) (PDLA), and poly(D,L-PLA) (PDLLA). PLLA and PDLA are semi-crystalline polymers while PDLLA is amorphous due to the random distribution of L-and D-lactide units. Both the crystallinity and mechanical properties of PLA can be tuned based on its molecular weight and the stereochemical makeup of its backbone[95]. High molecular weight PLA is usually prepared by the ring-opening polymerization method[96]. PLA undergoes hydrolytic degradation by the bulk erosion mechanism with the random scission of the main backbone and is more hydrophobic and the degradation time is long (15 years). In addition, the degradation byproduct lactic acid will also be broken down into water and carbon dioxide via the citric acid cycle[97]. PLA is widely used in many biomedical applications such as drug delivery or scaffolds for the regeneration of cardiac, nerve, and bone tissue as well as blood vessels[98-100]. In the case of fiber formation, PLA fibers have been fabricated via wet spinning, electrospinning, microfluidic spinning, melt spinning, and direct writing methods [101-103] 


\subsubsection{Poly(Lactide-co-Glycolide)}

Poly(Lactide-co-Glycolide) (PLGA) was developed as a family of copolymers with different lactide and glycolide compositions. PLGA is also synthesized by means of a ring-opening co-polymerization of two different monomers, glycolic acid, and lactic acid, and both L- and DL- lactides have been used for the copolymerization process. Gilding et al. have shown that compositions in the 25 to $75 \%$ range for Poly(L-Lactide-co-Glycolide) and 0 to $70 \%$ for the Poly(DLLactide-co-Glycolide) are amorphous[104]. PLGA has been shown to undergo bulk erosion through hydrolysis of the ester bonds, and the degradation rates are tunable by changing the ratio of PLA/PGA. Adding PGA into PLA will reduce the crystallinity of the copolymers, and therefore increase the degradation rate due to autocatalytic hydrolysis. For example, 50:50 Poly(DL-Lactide-co-Glycolide) degrades in 1-2 months, 75:25 Poly(DL-Lactide-co-Glycolide) in 4-5 months and 85/15 Poly(DL-Lactide-co-Glycolide) in 5-6 months[105]. The degradation byproduct of PLGA is poly( $\alpha$-hydroxy acids), which can cause local acidosis in the body[106]. PLGA has been approved by the Food and Drug Administration (FDA) for fiber-based scaffold applications, and various fiber formation techniques have been developed, such as direct writing, wet spinning, melt spinning, microfluidic spinning [107-109], and especially electrospinning methods [110-113].

\subsubsection{Poly( $\varepsilon$-caprolactone)}

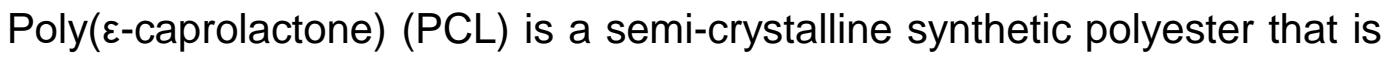
prepared through a ring opening $\varepsilon$-caprolactone polymerization scheme[114]. PCL has a low melting temperature $\left(58\right.$ to $\left.63^{\circ} \mathrm{C}\right)$, and is solube in a wide range of 
organic solvents. PCL degrades slowly ( $>2$ years) compared to other biopolymers through the hydrolysis of its aliphatic ester linkages. The degradation rate can be tailored by copolymerization with other lactones or glycolides/lactides. The good biocompatibility, mechanical properties, and processability make PCL desirable for tissue engineering applications, and more than 1500 papers have been published in the last two decades describing PCL-based biopolymers being used in biomedical engineering applications. In the case of fiber formation, PCL has been extensively studied for use in 3D scaffolds for tissue engineering with micro/nanoscale fibers being achieved through several techniques including direct writing, electrospinning, melt spinning, wet spinning and solvent casting [115-119]. Besides being used as single phase biopolymer, PCL is also a good base polymer for developing co-polymers or composites[120, 121].

\subsubsection{Composites}

As mentioned in the previous section, each of these individual biomaterial groups, including natural biopolymers and synthetic biopolymers, have their specific advantages and disadvantages (Table 2.4). A biopolymer composite is made by combining at least two constituent materials to produce a more viable scaffold by taking advantage of each composed material, independently. Unlike copolymers that are comprised of multiple different constituents, the phases of the composite material remain separate, and they are mechanically separable in theory, which allows for the transfer of loads between the different materials. The 
mechanical properties of composites can be tailored by carefully changing the volume fractions of the constituent materials[122].

Table 2. $4 \mathrm{~A}$ general comparison of natural and synthetic biopolymers in the scaffolding structure

\begin{tabular}{|c|c|c|}
\hline Biopolymer & Advantages & Disadvantages \\
\hline $\begin{array}{c}\text { Natural } \\
\text { biopolymer }\end{array}$ & $\begin{array}{l}\text { - Excellent biocompatibility, less } \\
\text { inflammatory or immunological } \\
\text { reaction } \\
\text { - Good cell attachment and signaling } \\
\text { due to biologically active } \\
\text { - Controlled enzymatic degradation } \\
\text { with no harmful degradation } \\
\text { product } \\
\text { - Usually highly porous and water- } \\
\text { soluble, and easy to get hydrogel }\end{array}$ & $\begin{array}{l}\text { - Slow production with batch-to- } \\
\text { batch variation } \\
\text { - May contain impurities or } \\
\text { heavy metal } \\
\text { - Mechanically weak } \\
\text { - Usually more expensive than } \\
\text { synthetic materials }\end{array}$ \\
\hline $\begin{array}{l}\text { Synthetic } \\
\text { biopolymer }\end{array}$ & $\begin{array}{l}\text { - Good biocompatibility } \\
\text { - Controlled hydrolytic degradation } \\
\text { - Predictable and tunable chemical } \\
\text { and physical properties, generally } \\
\text { better mechanical performance } \\
\text { - Large-scale production with batch- } \\
\text { to-batch uniformity } \\
\text { - Lower cost with a long shelf life }\end{array}$ & $\begin{array}{l}\text { - Biologically inert with poor cell } \\
\text { attachment due to the lack of } \\
\text { intrinsic surface ligands } \\
\text { - May induce toxic degradation } \\
\text { products } \\
\text { - Mostly hydrophobic and not } \\
\text { water soluble }\end{array}$ \\
\hline
\end{tabular}


Extensive research has been performed in developing natural-natural, synthetic-synthetic and natural-synthetic biopolymer composites for fiber or fibrous scaffold fabrication. For example, several composite fibers or fibrous scaffolds have been fabricated using fibrin and collagen[123], alginate and water-soluble chitin [84], PLGA with collagen[124], PLGA with chitosan[125], PCL and starch[126], and even silk fibroin, PLGA, and collagen[127]. As mentioned above, one of the major advantages of generating composite materials is to tailor the mechanical and/or chemical properties of the structure. Specifically, Slivka et al. combined PGA and PLGA fibers to enhance the compressive modulus and yield strength of articular cartilage scaffolds [128]. Similarly, Hokugo et al. produced a fiber hybrid sponge, for guiding skin and cartilage tissue formation, consisting of fibrin and PGA to yield a higher compressive modulus [129]. Another use of composites is to increase the bioactivity of the synthetic biopolymer through the introduction of natural biopolymers. Norouzi et al. demonstrated that hybrid scaffolds for skin regeneration and wound dressing applications can be created via co-electrospinning PLGA and gelatin improved fibroblast adhesion and proliferation[130]. Besides the previously mentioned blend spinning techniques of co-electrospinning and co-microfluidic spinning[131], another method to produce a composite material is by spinning fibers directly onto an existing matrix. For instance, Jeong et al. electrospun PLGA fibers onto the surface of tubular collagen scaffolds for vascular graft construction to improve the mechanical strength of the existing scaffold in both the dry and wet states[112]. 


\subsection{Fiber Fabrication Techniques}

The utilization of biopolymers and the ability to controllably fabricate micronand nano-sized fibers only recently appeared over the last few decades, which has opened the door for a wide variety of fiber-based tissue engineering approaches. Recently, several fiber-based fabrication techniques have been implemented to engineer 3D biomimetic tissue-like constructs with prescribed mechanical properties, topography and composition[132].

For microvascular structure formation, there are two common strategies: The first is to seed the cells in or on a fibrous scaffold and build a microvascular structure in situ. The other approach is to seed the cells directly onto or into a biodegradable fiber or fibrous scaffold that provides temporary mechanical support and create the inner lumen by removing the sacrificial structure. Both approaches are highly dependent on the fiber fabrication technique selected, which precisely controls the structural, topographical, mechanical properties, and degradation rates. In particular, the structural and topographical properties of the fibrous scaffold have been found to be essential for cell distribution[133], and the mechanical properties and degradation rate of the sacrificial fibers affect the cellular interaction and lumen formation[134].

We will introduce several existing methods for fabricating fibers from natural and synthetic biopolymers as well as composites while focusing on the current and/or potential application in the creation of microvascular structures. These fiber formation methods include (1) Electrospinning, (2) Microfluidic spinning, (3) Wet spinning, (4) Melt spinning, (5) Direct write/solvent casting, and (6) Others. 


\subsubsection{Electrospinning}

Electrospinning is a fiber spinning technique driven by a high-voltage, electrostatic field for drawing viscoelastic polymer fibers with diameters ranging from a few nanometers to few micrometers[135-138]. A typical electrospinning setup consists of a viscous polymer solution, a high voltage source (5-50 kV), a pumping system with a spinneret (e.g. a pipette tip), and a grounded metallic collector plate (e.g. metal screen, plate, or rotating mandrel) at an optimized distance from the pipette tip based on the particular setup (Figure 2.9)[137]. In the electrospinning process, the polymer solution is dispensed to the end of the spinneret to form a droplet due to surface tension. The electrostatic force opposes and eventually overcomes, the surface tension to eject a charged jet of the polymer solution from the spinneret tip. The solvent evaporates from the solution when the jet travels from the tip to the collector, eventually forming a continuous fibrous mat on the collector[137]. The polymer solution properties (i.e., viscosity, surface tension, and electrical conductivity), the flow rate, applied voltage and distance of spinneret from collector directly affect the microstructure of the fibrous mat [139]. In addition, fibrous mats with random oriented or aligned fibers can be controlled by changing a stationary collector to a rotating one[140]. 


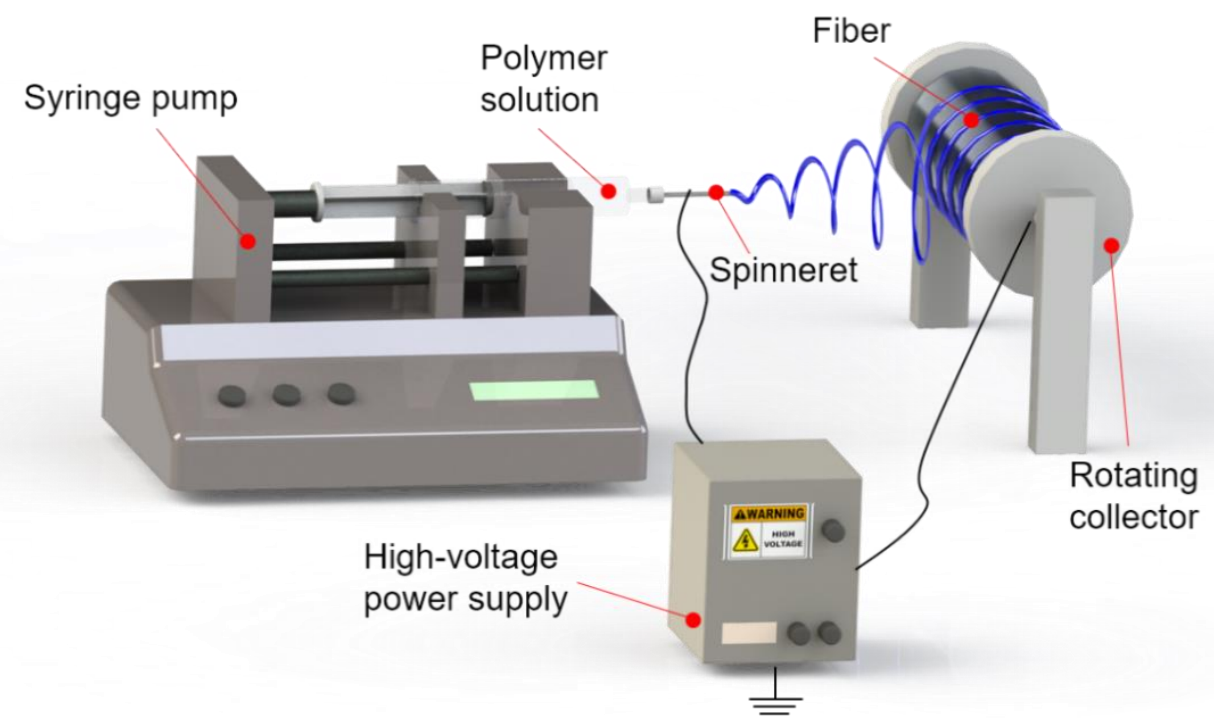

Figure 2. 9 Schematic diagram of set up of electrospinning [141].

Electrospinning is a versatile and relatively simple technique that can be used to process many biopolymers into fibers. In fact, electrospinning is one of the most commonly used scaffold fabrication techniques because it can easily produce a nanofibrous mat with an ECM-like architecture. You et al. constructed nanofibrous scaffolds from PGA, PLA, and PLGA and evaluated the biodegradation rate of these scaffolds[140]. Fioretta et al. investigated the impact of different fiber diameters $(2,5,8$, and $11 \mu \mathrm{m})$ in electrospun PCL scaffolds on endothelial colony forming cells (ECFCs) in comparison to mature human umbilical vein endothelial cells (HUVECs). The results suggested that the individual fiber diameters of the fibrous scaffold can determine cell phenotype[115].

Some researchers have indicated that aligned electrospun ultrafine fibers can regulate cellular alignment and relevant functional expression. Dalton et al. 
electrospun fibers with diameters of $\sim 960 \mathrm{~nm}$ from blends of PEG and PCL and patterned them into aligned lines and seeded with fibroblasts. The seeded fibroblast morphology was observed to be affected by the fiber orientation[142]. Zhang et al. prepared hydrogel fibers with uniaxial alignment from aqueous solutions of natural polymers such as alginate, fibrin, gelatin, and hyaluronic acid using an electrospinning technique in combination with electrical and mechanical stretching. The internal alignment feature enhanced the mechanical properties of the hydrogel microfibers and induced HUVEC alignment[143]. The fiber topography was also shown to affect cell behavior. Santos et al. electrospun PCL nanofibrous meshes on fibronectin-coated PCL fibers (160 nm in diameters) for endothelial cell migration in bone tissue and discovered that the ECs expressed a more elongated phenotype when compared to the PCL fibers with no nanofiber mesh[144]. Ekaputra et al. utilized a dual electrospinning /electro-spraying setup to fabricate a scaffold containing PCL fibers, collagen, and a hyaluronic acid-based hydrogel. This multi-component design encouraged osteoblasts to penetrate the scaffold, rather than simply growing across its surface[145].

With respect to vascular constructs, Xu et al. used a rotating collector to produce an aligned nanofibrous mat of poly(I-lactide-co-e-caprolactone) $[\mathrm{P}(\mathrm{LLA}$ $\mathrm{CL})$ ] (75:25) copolymer with the average diameter of the aligned fibers being 550 $\mathrm{nm}$ in order to create a scaffold for potential use as a blood vessel scaffold. Smooth muscle cells (SMCs) were seeded onto the scaffold, and SMC cytoskeletal proteins were found to align in the direction of the scaffold fibers[146]. Similarly, Zhu et al. employed a mandrel collector to fabricate porous fiber meshes of 
macroscopically aligned PCL fibers with an average diameter of $\sim 10 \mu \mathrm{m}$ for vascular tissue engineering applications. Human umbilical artery smooth muscle cells and HUVECs were seeded and cultured on these scaffolds[147]. Zhou et al. fabricated aligned PLLA microfibers with an average fiber diameter of $1.6 \mu \mathrm{m}$ by using a jet electrospinning method. Ellipse-shaped nano-pores were incorporated in situ onto individual fiber surfaces to form nano-topographical features by varying ambient humidity. A 7-day in vitro assessment of human vascular smooth muscle cells (vSMCs) cultured on these fibers indicated correlations with the nanoroughness[102].

Recently, electrospun fiber-based scaffolds have been improved by incorporating other components to obtain a bioactive coating or hybrid scaffold. Kwon et al. co-electrospun poly(L-lactide-co-caprolactone) (PLCL) with type I collagen and demonstrated that HUVECs exhibited improved attachment on the scaffold relative to a collagen-free scaffold[148]. Jeong et al. co-cultured SMCs and ECs onto a similar hybrid scaffold composed of a porous collagen matrix and an electrospun fibrous PLGA layer for vascular graft applications [112]. Likewise, Barreto-Ortiz et al. electrospun microfibers (average diameter $200 \mu \mathrm{m}$ ) composed of a fibrin-coated hydrogel mixed with alginate and demonstrated that these microfibers could be used to guide the stepwise formation of multicellular microvascular structures comprised of ECFCs, vSMCs, and pericytes[149]. 


\subsubsection{Microfluidic Spinning}

Microfluidic spinning, also known as co-axial flow spinning, is a fiber formation technique based on micro-fluid dynamics principles. A typical microfluidic spinning system is comprised of a central channel, which delivers the polymer solution (sample flow) into the main channel, and two side channels that deliver sheath flow around the sample flow (Figure 2.10)[132, 150]. Due to the laminar nature of the flow in the microfluidic channel, the interface between the sheath flows and sample flow remains stable and polymerization only occurs downstream by either UV light exposure, ionic or chemical crosslinking process or a solvent exchange method[150]. The fiber diameter and cross-sectional shape are tunable by changing the polymer solution viscosity, the ratio between the sample flow and sheath flow rates and the channel geometry[151, 152]. Fibers with aligned orientation can be achieved by utilizing a rotating roller collector[153].

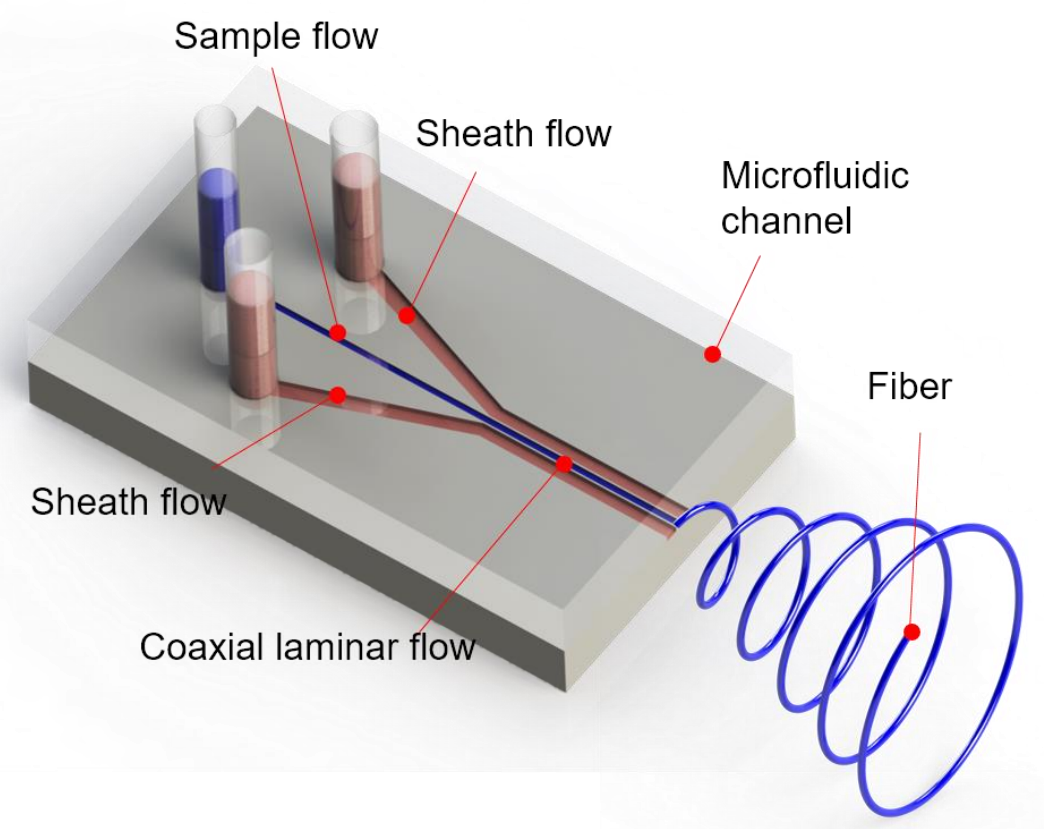


Figure 2. 10 Schematic diagram of set up of microfluidic spinning [150]. In this process, fiber solidification can be achieved by either a solvent method or nonsolvent method, including photopolymerization, ionic crosslinking, solvent exchanging and chemical crosslinking.

One major advantage to this technique is that the sample flow can be loaded with cells, which can remain viable and functional because the microfluidic channels are usually short in length and the cells are only exposed to a high shear stress for a very short period of time. Despite numerous advantages offered by microfluidic spinning, there are downsides to this technique. For example, the short amount of time available to solidify the fibers limits the choice of materials. Until now, only a handful of biopolymers have been used to produce microfibers through this technique. In addition, the microchannel can easily clog, which significantly affects yield. In addition, the microfluid spinning technique lacks the ability to generate cylindrical shapes that are similar to in vivo environment.

Nonetheless, microfluidic spinning is emerging as a promising method for producing continuous fibers from biopolymer solutions. Reviews on general tissue engineering applications of this technique are available elsewhere[66, 133, 150, 154]. Additionally, several investigators have developed microfluidic spinning to develop models for better understanding of neuronal and cancer growth, which also have the potential for being used in the construction of microvascular structures. Specifically, Kang et al. employed a PDMS microfluidic platform to continuously generate alginate thin flat fibers (thickness $<10 \mu \mathrm{m}$ ) with engraved 
grooved patterns that were used to align different cells, such as cortical neuronal cell and myoblast cells[87]. Similarly, Hwang et al. designed a PDMS-based microfluidic spinning device to produce PLGA microfibers with diameters ranging from 20 to $230 \mu \mathrm{m}$ and demonstrated the ability to culture aligned L929 fibroblasts [108]. Wei et al. produced microfluidic-based cell-laden microfibers from UV-crosslinkable methacrylated alginate and HUVECs and MG63 cells[155]. In another study, a collagen suspension of Hep-G2 and HUVECs hydrogel microfibers were processed into microfibers (diameter $\sim 230 \mu \mathrm{m}$ ) by Stato et al. via a double coreshell type microfluidic device and confirmed the connection of the cells by cultivation[156].

For microvascular structure formation, Tung et al. fabricated microvascular scaffold composed of PLGA fibers using a PDMS replica mold. These PLGA fibers featured diameters ranging from 13-50 $\mu \mathrm{m}$ that were wrapped with HUVECs on both sides[157]. Daniele et al. utilized poly(ethylene glycol) dimethacrylate (PEGDMA) and gelatin to create a variety of microvascular structures, including microfibers, microtubes, coaxial microfibers, and triaxial microfibers[131]. In a more recent study, Tian et al. presented a gas-in water microfluidic method to fabricate alginate-based composite microfibers with "cavity knots" and assembled them into a 3D scaffold, in which HUVECs were cultured to create a vascular structure[158]. Cell-laden fibers can also be produced by microfluidic spinning technique. For example, Lee et al. used a microfluidic chip and a mixture of Human Iliac Vein Endothelial-78 cells (HIVE-78) and 2 wt.\% alginate solution to generate hollow alginate cell-laden fibers. Then they embedded these cell-laden fibers into 
hydrogels with smooth muscle cells and successfully cocultured these cells for seven days[159].

\subsubsection{Wet Spinning}

Wet spinning is a non-solvent-induced, phase-inversion, fiber fabrication technique that can yield microfibers with a wide range of diameters[160]. A standard wet spinning setup includes an injection system (can be driven manually, gravitationally, or by a syringe pump), a reservoir for the polymeric solution, a spinneret and a coagulation bath (which must contain either a poor solvent or nonsolvent for the polymers)(Figure 2.11)[132, 161, 162]. During spinning, the polymeric solution is continuously injected into one or multiple coagulation baths and the long continuous filament solidifies due to the polymer and solvent/nonsolvent exchange[161]. Some improved wet spinning systems introduce a fiber collection component such as rotating mandrel to attain circumferentially oriented microfiber scaffolds[163, 164]. 


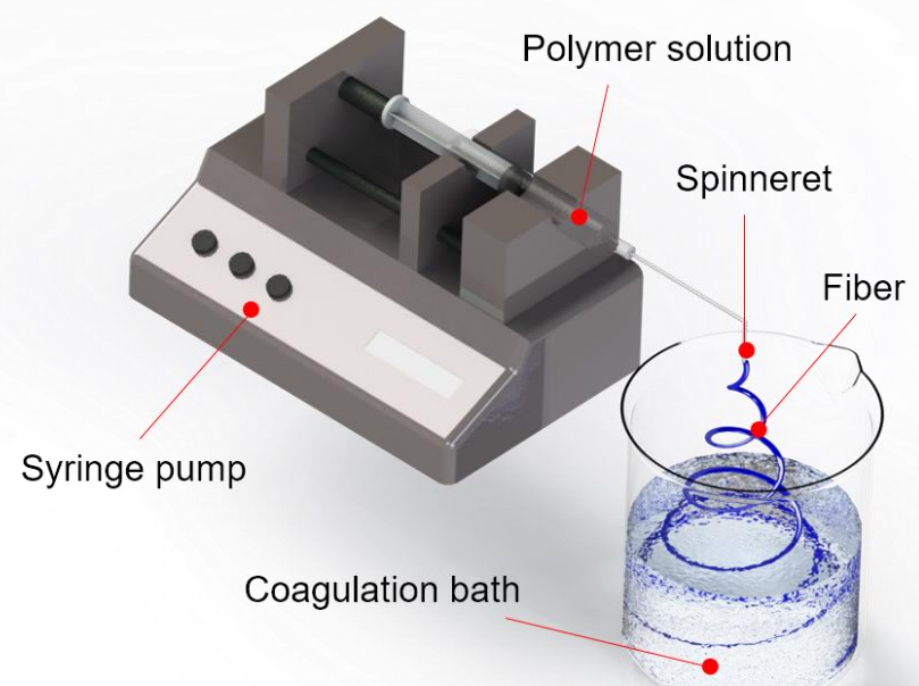

Figure 2. 11 Schematic diagram of set up of wet spinning [162]

While wet spinning is not able to produce scaffolds with ultra-small sized fiber diameters, in general, like electrospinning and microfluidic spinning, the fiber diameter is still similarly controlled by the polymer viscosity, the injection rate and the spinneret size[165]. One advantage of wet spinning scaffolds is that they have larger pore sizes compared to those fabricated by electrospinning, which is favorable for cell adhesion and cellular penetration within the scaffold[116, 166]. Wet spinning can also incorporate of cells within the fibers just like microfluidic spinning. However, the cross-linking reagents must be cell friendly because the cell exposure time is much longer than microfluidic spinning.

Wet spinning is a straightforward and very easy to set up, and it has the capability of high volume fiber production if multiple spinnerets are used. A variety of natural biopolymers, synthetic biopolymer, and the composites thereof have 
been used to generate micron size fiber-based scaffolds[132, 133]. In the case of microvascular structure applications, Zhang et al. developed a new wet spinning system to fabricate oriented PCL microfiber scaffolds with the fiber diameter and porosity being controlled in the range of $7-27 \mu \mathrm{m}$ and 68 to $82 \%$, respectively. SMCs could grow in an oriented fashion along the fibers and infiltrate inside the scaffold[116]. Takei et al. extruded sodium alginate solution containing bovine carotid artery vascular endothelial cells (BECs) into $\mathrm{CaCl}_{2}$ solution and obtained microfibers. The BECs eventually migrated into the ambient collagen gel and selfassembled into capillary-like structures[88]. Microvascular networks could also be created by wet spinning sacrificial microfibers. In a recent study, Lee et al. reported a sacrificial template-based strategy utilizing wet spun poly( $\mathrm{N}$-isopropylacrylamide) (PNIPAM) fibers (diameters ranging from 3-55 $\mu \mathrm{m}$ ) to produce 3D microvascular networks in cell-laden gelatin hydrogels. This capillary-like microvascular network allowed constant perfusion of the media and improved the viability of human neonatal dermal fibroblasts encapsulated within the gel[167].

\subsubsection{Melt Spinning}

Melt spinning, sometimes called extrusion, is widely used for fiber formation in the polymer industry. In the standard process of melt spinning, a polymer is heated to its melting point and extruded through a micron-sized spinneret orifice and then directly solidified into continuous fibers by rapid cooling (Figure 2.12) [133]. In general, melt spinning tends to produce the largest diameter fibers (on the order of a few hundred microns) compared to the previously mentioned fiber 
fabrication techniques. However, melt spun fibers with a diameter under $100 \mu \mathrm{m}$ have also been reported[168]. The fibers' diameter is affected by the polymer's characteristic properties, size of the spinneret orifice, spinning temperature, and extrusion rate[169]. Melt spinning has a unique advantage in its ability to create controlled, complex cross-sections of the fibers. Star shape, fractal-like, grooved and even hollow fibers have been produced by melt spinning with special designed spinneret orifices [170-173]. Researchers have shown fibers with grooved crosssections assist in cells attaching and aligning themselves parallel to the direction of the grooves[171]. Associated results also support that the mechanical properties such as tensile strength and Young's modulus are improved by melt spinning[101]. However, due to the spinning process requiring high temperatures, it is challenging to incorporate cells into the melt spinning fiber process like microfluidic spinning and wet spinning.

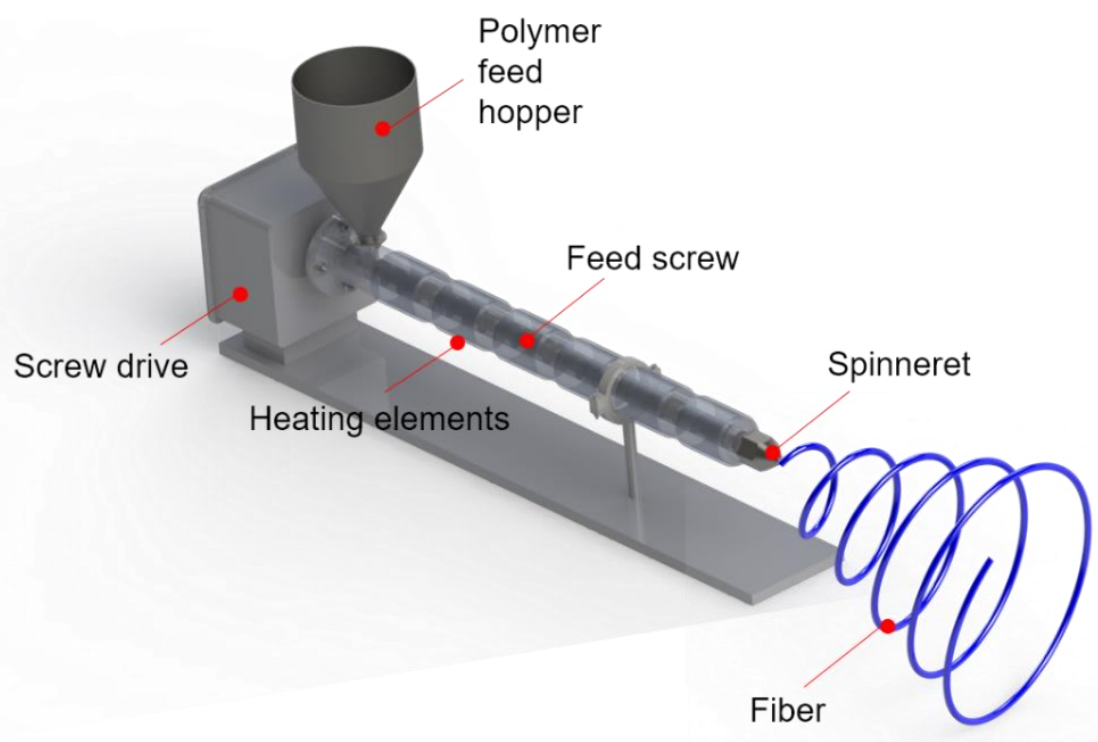

Figure 2. 12 Schematic diagram of set up of melt spinning [133] 
Melt spinning was first used to produce fibers in the textile industry and now it is an appeal in the creation of biopolymer fibers has grown recently in the tissue engineering arena. Park et al. utilized three customized spinnerets to produce PCL fibers with circular, triangular, and cruciform cross-section and the equivalent diameters of $\sim 200 \mu \mathrm{m}$. They further showed the woven scaffolds composed of the fibers with noncircular cross-section significantly increased the proliferation of human osteosarcoma MG63 cells and decreased the degradation time[117]. Wu et al. assembled melt spun PGA-PLLA fibers into a non-woven scaffold and showed that human endothelial progenitor cells (EPC) have an inherent ability to assemble into a microvascular-like network[174]. Similarly, melt spun fibers were used as sacrificial fibers to create 3D microvascular networks. Specifically, Bellan et al. used cotton candy to fabricate sugar fibers and then developed a 3D perfusable microvascular network using the sugar fibers as sacrificial structures[134]. In a more recent study, Patrick et al. developed a 3D interconnected microvasculature in which the sacrificial fiber composed of PLA and tin(II) oxalate was melt spun with diameters of the composites fibers ranging from 550 to $850 \mu \mathrm{m}[175]$.

\subsubsection{Direct Write/Solvent Casting}

Traditional solvent casting is a fiber manufacturing process that involves mixing of a solubilized polymer matrix and filler under continuous agitation through mechanical stirring, followed by casting and solvent evaporation or drying steps[176]. In this straightforward process, the polymer is first dissolved in an 
appropriate volatile solvent or water, and then the polymer solution is cast on a substrate such as a flat surface or heated drum. The traditional process does not need special equipment and is very easy to implement, and thus, has become popular for biological applications. For example, Lieder et al. developed a protocol for solvent casting chitosan membranes and demonstrated that the mouse preosteoblastic cell line MC3T3-E1 could be successfully attached for 24 days[78].

Overall, the solvent casting method is an inherently slow process, but the concept itself offers potential when adapted to micro- and nano-scale applications using specialized equipment. Specifically, a technique known as direct write was recently developed to create a fiber-based scaffold for tissue engineering and microvascular network applications[177, 178]. A typical direct write setup includes a computer-controlled translation stage, a deposition nozzle, and a deposition substrate (Figure 2.13). Nain et al. and Berry et al. have produced polymer fibers with micro- and nanoscale diameters by directly ejecting polymer solution from a hollow capillary and then thinning into filaments by capitalizing on the surface tension-driven necking phenomenon, which like solvent casting leverages the solvent volatility to form fibers $[179,180]$. Berry et al. further showed that the fiber diameter could be controlled by solution concentration, drawing rate, and fiber length[181]. They also demonstrated the ability to generate micron-sized fibers from a variety of biopolymers such as PCL, PLLA, PDLLA, and DL-PLGA[181]. Similarly, Vozzi et al. have utilized a pressure assisted micro-syringe to direct write PLGA scaffolds with feature sizes of $10-30 \mu \mathrm{m}[111]$. Guo et al. have reported a robotic system to print various geometries such as filaments, towers, and freeform 
circular spirals from PLA[182, 183]. In another study, Kullenberg et al. have employed a pressure-assisted micro-syringe (PAM) system to fabricate hexagonal scaffolds of PLGA fibers and discovered the optimized hexagonal scaffold size for neural cell adhesion[184]. Nain et al. have developed a Spinneret-Based Tunable Engineered Parameters (STEP) technique to construct aligned microfibers using both PLA and PLGA. They further investigated the effect of the scaffold morphology to the mouse C2C12 cellular behavior[185]. Similarly, Wang et al. fabricated suspended multilayer hierarchical nanofiber scaffold using STEP technique and seeded C2C12 mouse myoblasts on the scaffold[186].

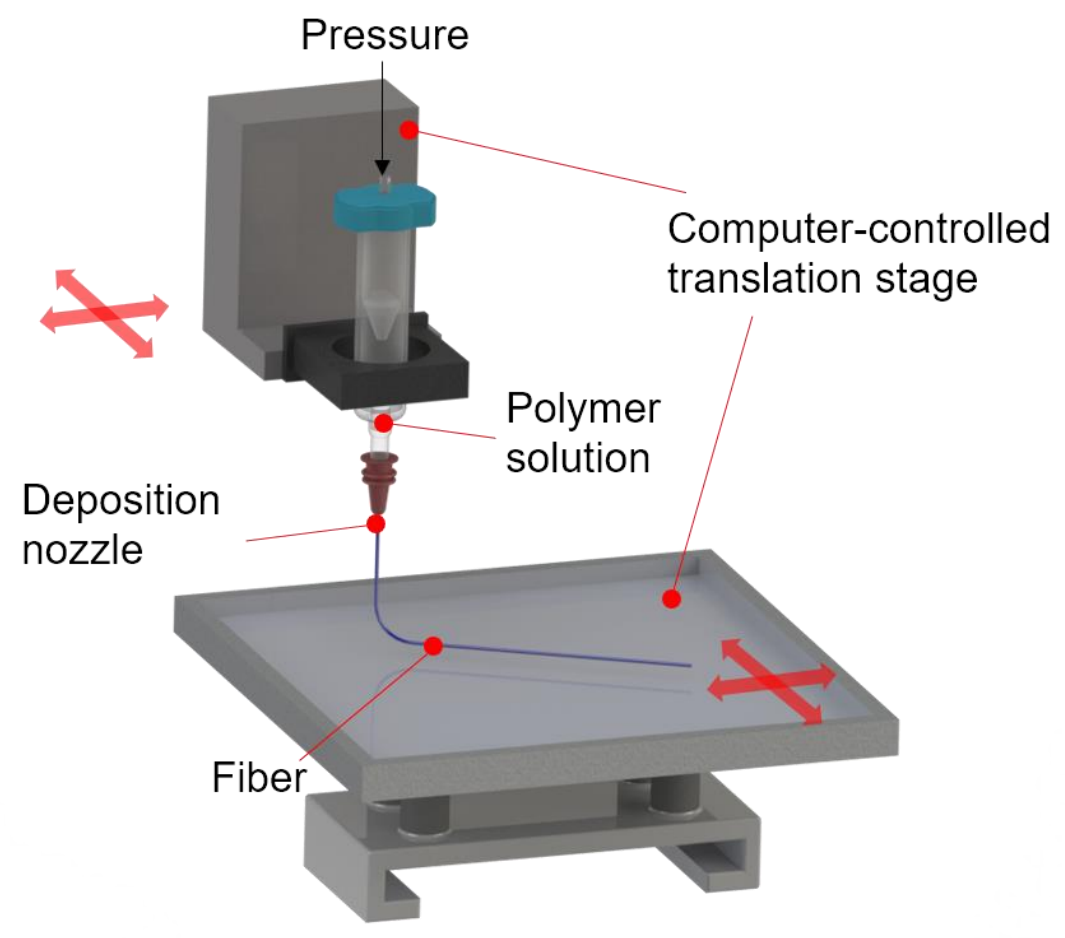

Figure 2. 13 Schematic diagram of set up of direct write 
The direct write technique offers more dimensional and textural control compared to other fiber spinning techniques. More recently, Berry et al. created microvascular scaffolds using the direct write technique and successfully seeded the fibers with HUVECs. These scaffolds were created as either individual fiber strands or with branched structures composed of suspended synthetic biopolymer (L-PLA, DL-PLA, PLGA (50:50 and 75:25) and PCL) microfibers with microvascularscale diameters $(5-20 \mu \mathrm{m})$ and point-to-point orientation[118]. Direct write technique can also incorporate cells within the fibers and produce cell-laden fibers for microvascular structures. Gaetani et al. have used a bioprinting system and a mixture of HUVECs and alginate to form an endothelialized micro-fibrous scaffold[89].

\subsubsection{Others}

In addition to the five previously mentioned fiber fabrication techniques, some other polymer fiber-based scaffold methodologies have been implemented to produce cellular scaffolds. Although these scaffolds are not all used for creating microvascular structures, these methodologies still hold promise for future applications. For example, Interfacial Polyelectrolyte Complexation(IPC) is a process whereby fibers and capsules are formed through interactions at the interface of oppositely charged polymers[187]. One significant advantage of the IPC process is the ability to encapsulate biological components such as cells, extracellular matrix proteins, growth factors, and plasmid DNA. Du et al. have developed a method to draw IPC fibers and encapsulate hepatic and endothelial 
cells into the fibers to assemble endothelialized liver tissue constructs[188]. Human mesenchymal stem cells (hMSCs) encapsulated in IPC fibers made from alginate and water-soluble chitin polymers have been reported by Yim et al.[84]. Similarly, Lu et al. used a microfiber system to fabricate IPC fibers for human pluripotent stem cell (hPSC) encapsulation[189]. More recently, Lim et al. used the same polymers to generate 3D fibrous hydrogel cell-laden scaffolds for engineered follicular structures[190]. In general, the IPC technique requires a relatively simple setup; however, both the biopolymer selection available for this process and the working fiber diameter range is limited.

Rotary Jet Spinning(RJS) is another reproducible technique for bulk nano/micro-fiber production. In this process, the polymer solution is ejected from the micron-sized orifice in a high-speed rotating reservoir, and nanoscale polymer fibers are solidified on the collector by solvent evaporation. Golecki et al. have fabricated PLA nanofibers with a diameter ranging from 250 to $950 \mathrm{~nm}$ and revealed that fiber drying played an important role in determining nanofiber morphology[191]. In another study, Badrossamay et al. utilized PLA/chloroform solution to fabricated aligned 3D nanofiber structures with diameters ranging from 50 to $3500 \mathrm{~nm}$, and they further created anisotropic muscle with aligned and elongated myocytes and ordered myofibrils, based on the nanofibers[192].

A variety of rarely utilized fiber formation techniques have also been reported for tissue engineering applications. Qiu et al. found that polyvinylpyrrolidone (PVP) could self-assemble into branched hollow fibers, which can potentially be applied to forming artificial blood vessels[193]. Wang et al. used 
femtosecond laser ablation to fabricate pillared PLGA microvessels and observed that bovine endothelial cells adhered well and grew to surround each branch of the pillared microvessel network[194]. In recent research, Kalisky et al. have presented a method to produce microfibers $(\sim 50 \mu \mathrm{m})$ using a core-shell approach, composed of a calcium alginate shell and a cellularized type I collagen core. This approach can control fiber diameter and geometry, and it also allows cultured cells to distribute preferentially on the surface of the fiber and display a uniform cellular orientation[83].

A comparison of the biopolymer fabrication techniques is shown in Table 2.5. The Summary of recent studies on microvascular structures using the different fiber fabrication techniques and cell types is shown in Table 2.6.

Table 2. 5 Comparison of different biopolymer fabrication techniques

\begin{tabular}{|c|c|c|}
\hline $\begin{array}{l}\text { Fiber } \\
\text { fabrication } \\
\text { technique }\end{array}$ & Advantages & Disadvantages \\
\hline Electrospinning & $\begin{array}{l}\text { - Multiple biopolymer choices } \\
\text { - Ability to form nano-scale fibers } \\
\text { - Volume production }\end{array}$ & $\begin{array}{l}\text { - Not suitable for cell } \\
\text { encapsulation due to harsh } \\
\text { process } \\
\text { - Difficult to fabricate single fiber } \\
\text { or spatially controlled structure } \\
\text { - Difficult to form a thick } 3 D \\
\text { structure }\end{array}$ \\
\hline
\end{tabular}




\begin{tabular}{|c|c|c|}
\hline $\begin{array}{l}\text { Microfluidic } \\
\text { spinning }\end{array}$ & $\begin{array}{l}\text { - Ability to control fiber cross- } \\
\text { sectional shape and diameter } \\
\text { - Tiny volume sample } \\
\text { consumption } \\
\text { - Suitable for cell encapsulation } \\
\text { - Ability to get hollow or coaxial } \\
\text { fibers }\end{array}$ & $\begin{array}{l}\text { - Complicated setup } \\
\text { - Limited biopolymer choices } \\
\text { - Microchannel clogging }\end{array}$ \\
\hline Wet spinning & $\begin{array}{l}\text { - Pre-controlled fiber diameter } \\
\text { - Suitable for cell encapsulation }\end{array}$ & $\begin{array}{l}\text { - Unable to form nano-scale } \\
\text { fibers } \\
\text { - Difficult to get aligned fibers }\end{array}$ \\
\hline Melt spinning & $\begin{array}{l}\text { - Ability to control fiber cross- } \\
\text { sectional shape and diameter } \\
\text { - Simple setup }\end{array}$ & $\begin{array}{l}\text { - Not suitable for cell } \\
\text { encapsulation due to high } \\
\text { temperature } \\
\text { - Limited to large fiber diameters }\end{array}$ \\
\hline Solvent casting & $\begin{array}{l}\text { - Ease of fabrication without the } \\
\text { need for specialized equipment }\end{array}$ & $\begin{array}{l}\text { - Limited biopolymer choices } \\
\text { - Unable to control fiber } \\
\text { alignment } \\
\text { - Slow process } \\
\text { - Limited to large fiber diameters }\end{array}$ \\
\hline Direct write & $\begin{array}{l}\text { - Ability to control fiber } \\
\text { orientation and diameter } \\
\text { - Ability to form nano-scale fibers } \\
\text { - Suitable for cell encapsulation }\end{array}$ & $\begin{array}{l}\text { - Complicated setup (i.e. } \\
\text { computer-controlled translation } \\
\text { stage) } \\
\text { - Difficult for volume production }\end{array}$ \\
\hline
\end{tabular}




\begin{tabular}{c|l|l}
\hline & & \\
\hline Interfacial & - Suitable for cell encapsulation & - Limited biopolymer choices \\
polyelectrolyte & fibers & - Unable to control the fiber \\
complexation & & alignment \\
& & \\
\hline
\end{tabular}

Table 2. 6 Summary of recent studies on microvascular structures using the different fiber fabrication techniques and cell types

Fiber

Fabrication Biopolymers

Technique

\section{Fiber}

Diameter
Cell Type

Ref.

\begin{tabular}{lllll}
\hline PCL & $2-11 \mu \mathrm{m}$ & ECFCs, HUVECs & {$[115]$} \\
& PCL-PLA & & & \\
copolymer & $550 \mathrm{~nm}$ & SMCs & {$[146]$} \\
& PCL & $10 \mu \mathrm{m}$ & SMCs and HUVECs & {$[147]$} \\
Electrospinning & PEG and PCL & $960 \mathrm{~nm}$ & Fibroblasts & {$[142]$} \\
& Alginate, fibrin, & & & \\
& gelatin, and & $10-25 \mu \mathrm{m}$ & HUVECs & {$[143]$} \\
& hyaluronic acid & & & \\
& & & & {$[102]$}
\end{tabular}


PLCL and

120-520 nm HUVECs

[148]

collagen

PLGA Submicron SMCs and ECs

[112]

Fibrin hydrogel

mixed with

alginate

ECFCs, vSMCs,

$200 \mu \mathrm{m}$

and pericytes

PCL $\quad 160 \mathrm{~nm} \quad$ HUVECs

[144]

PCL $\quad 1-2 \mu \mathrm{m} \quad$ Osteoblasts

[145]

PLGA

$\sim 10 \mu \mathrm{m} \quad$ HUVECs

[195]

PLGA

$20-230 \mu \mathrm{m}$

L929 fibroblast

[108]

Cortical neuronal

\begin{tabular}{|c|c|c|c|}
\hline & Alginate & $<10 \mu \mathrm{m}$ & $\begin{array}{l}\text { cell and myoblast } \\
\text { cells }\end{array}$ \\
\hline & Chitosan & $50-200 \mu \mathrm{m}$ & Fibroblast \\
\hline Microfluidic & Calci $\mu m$ alginate & $19 \mu \mathrm{m}$ & Fibroblast \\
\hline & Calcijm alginate & $40-300 \mu \mathrm{m}$ & $\begin{array}{l}\text { HepG2 and NIH } \\
\text { 3T3 }\end{array}$ \\
\hline & PLGA & $13-50 \mu \mathrm{m}$ & HUVECs \\
\hline & $\begin{array}{l}\text { PEGDMA and } \\
\text { gelatin }\end{array}$ & $<500 \mu \mathrm{m}$ & - \\
\hline
\end{tabular}


Alginate-based

composite $\quad \sim 100 \mu \mathrm{m} \quad$ HUVECs

[158]

solution

Alginate $\quad 40-220 \mu \mathrm{m} \quad$ HIVE-78, SMCs [159]

Alginate

HUVECs and MG63

\begin{tabular}{lll} 
Alginate & $\sim 450 \mu \mathrm{m}$ & cells \\
& & \\
Collagen & $\sim 230 \mu \mathrm{m}$ & HUVECs \\
\hline PCL & $7-27 \mu \mathrm{m}$ & SMCs \\
& & \\
Sodium alginate & $250-500 \mu \mathrm{m}$ & BECs
\end{tabular}

Wet spinning

PCL

Fibroblasts and

myoblasts

\begin{tabular}{|c|c|c|c|c|}
\hline & PCL & $150 \mu \mathrm{m}$ & myoblasts & [119] \\
\hline & PNIPAM & $3-55 \mu \mathrm{m}$ & Fibroblasts & [167] \\
\hline \multirow{6}{*}{ Melt spinning } & PCL & $\sim 200 \mu \mathrm{m}$ & MG63 cells & [117] \\
\hline & PGA-PLLA & - & EPC & [174] \\
\hline & Collagen & $200-300 \mu \mathrm{m}$ & - & [70] \\
\hline & Chitosan & 286-352 $\mu \mathrm{m}$ & VICs & [79] \\
\hline & Cotton sugar & $20 \mu \mathrm{m}$ & - & [134] \\
\hline & $\begin{array}{l}\text { PLA and } \operatorname{tin}(\mathrm{II}) \\
\text { oxalate }\end{array}$ & $550-850 \mu \mathrm{m}$ & - & [175] \\
\hline
\end{tabular}




\begin{tabular}{|c|c|c|c|c|}
\hline & & & Pre-osteoblastic & \\
\hline & Chitosan & - & $\begin{array}{l}\text { cell line MC3T3- } \\
\text { E1 }\end{array}$ & [78] \\
\hline & $\begin{array}{l}\text { PMMA, PCL, } \\
\text { PLLA, PDLLA, } \\
\text { and PLGA }\end{array}$ & $1-100 \mu \mathrm{m}$ & - & [181] \\
\hline & PLGA & $10-30 \mu \mathrm{m}$ & - & [111] \\
\hline & PLA & $80 \mu \mathrm{m}$ & - & [182] \\
\hline & PLGA & $10-30 \mu \mathrm{m}$ & SH-SY5Y cell line & [184] \\
\hline Solvent casting & PLA and PLGA & 50 to $500 \mathrm{~nm}$ & $\begin{array}{l}\text { C2C12 mouse cell } \\
\text { line }\end{array}$ & [185] \\
\hline & Polystyrene & $\begin{array}{l}100 \mathrm{~nm} \text { to } \\
\text { micrometers }\end{array}$ & $\begin{array}{l}\mathrm{C} 2 \mathrm{C} 12 \text { mouse } \\
\text { myoblasts }\end{array}$ & [186] \\
\hline & $\begin{array}{l}\text { PCL, PLLA, } \\
\text { PDLLA and PLGA }\end{array}$ & $5-20 \mu \mathrm{m}$ & HUVECS & [118] \\
\hline & Alginate & - & $\begin{array}{l}\text { Human cardiac- } \\
\text { derived } \\
\text { cardiomyocyte } \\
\text { progenitor cells }\end{array}$ & [89] \\
\hline & Fibrin & $93 \mu \mathrm{m}$ & HMVECS & [32] \\
\hline
\end{tabular}




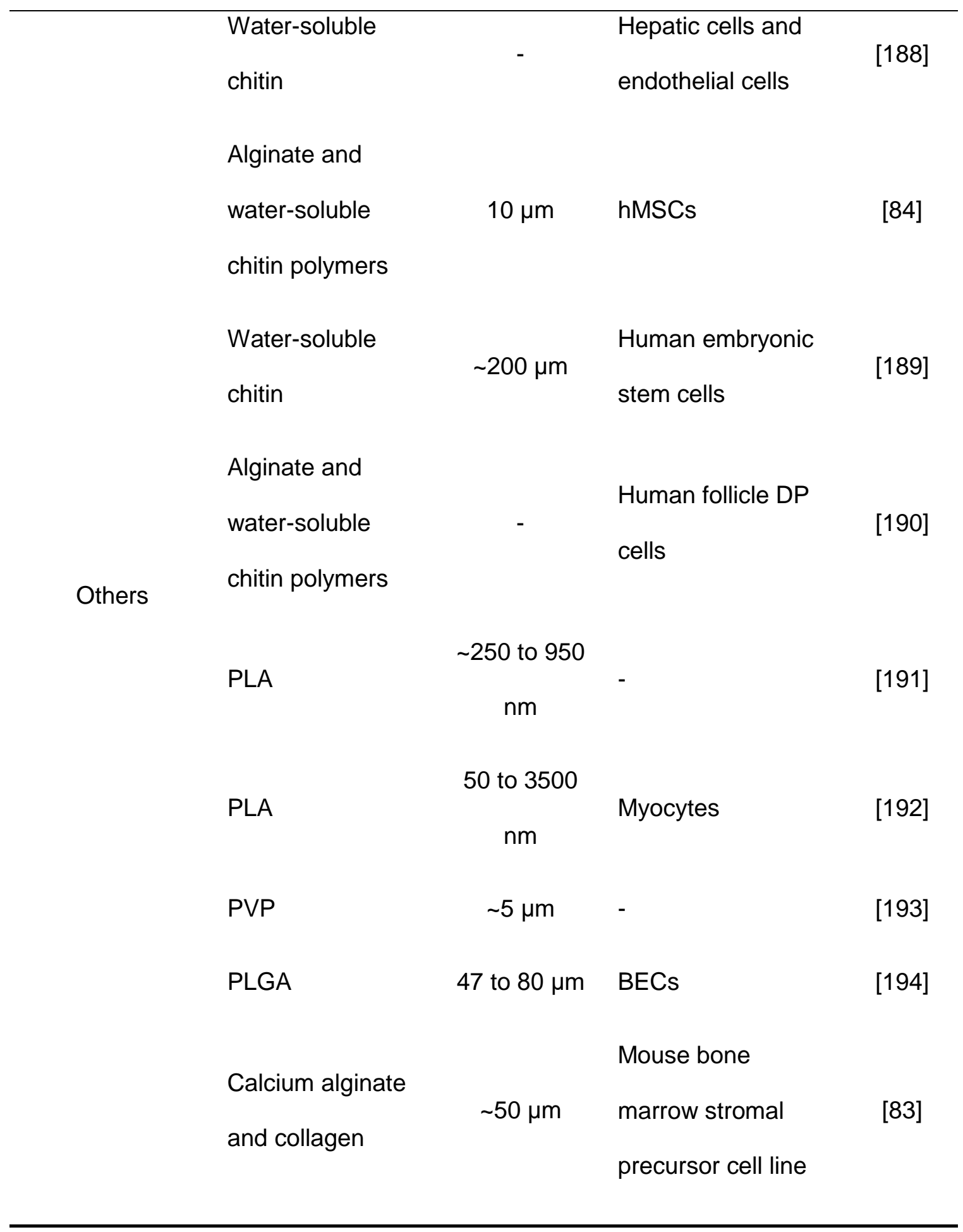




\section{CHAPTER 3: MATERIALS AND METHODS}

\subsection{Overview}

In this chapter, the instrumentation and techniques used to fabricate precisely positioned, three-dimensional, suspended branched microvascular fiber scaffolds and form a confluent monolayer of Human Dermal Microvascular Endothelial Cells (HDMECs) on the scaffold are described.

The schematic illustrating the formation of a microvascular network is shown in Figure 3.1. The fabrication process consisted of implementing a 3-Axis robotic dispensing system for creating a suspended, branched micro-fiber scaffold (Figure 3.1A). The direct-write system was able to predictably and repeatedly construct micro-fibers with microvascular-scale diameters $(5-20 \mu \mathrm{m})$. An empirical model was generated based on the gelatin fiber characterization data to express micro-fiber diameter in terms of the direct-write system operational parameters and polymer solution properties. The empirical model was validated and tested by drawing fibers from various biopolymers, composites, and copolymers. Upon completion of the fabrication process, HDMECs were seeded on the scaffold and cultured for two days. The cells wrapped circumferentially around the scaffold and spread along the axial direction of the scaffold (Figure 3.1B-C). The HDMECs-covered scaffold was subsequently cultured in a labderived extracellular matrix (ECM) for an additional three days. The ultimate vision 
is that the cells can attach to the ECM and maintain the lumen shape after the scaffold is degraded (Figure 3.1D).
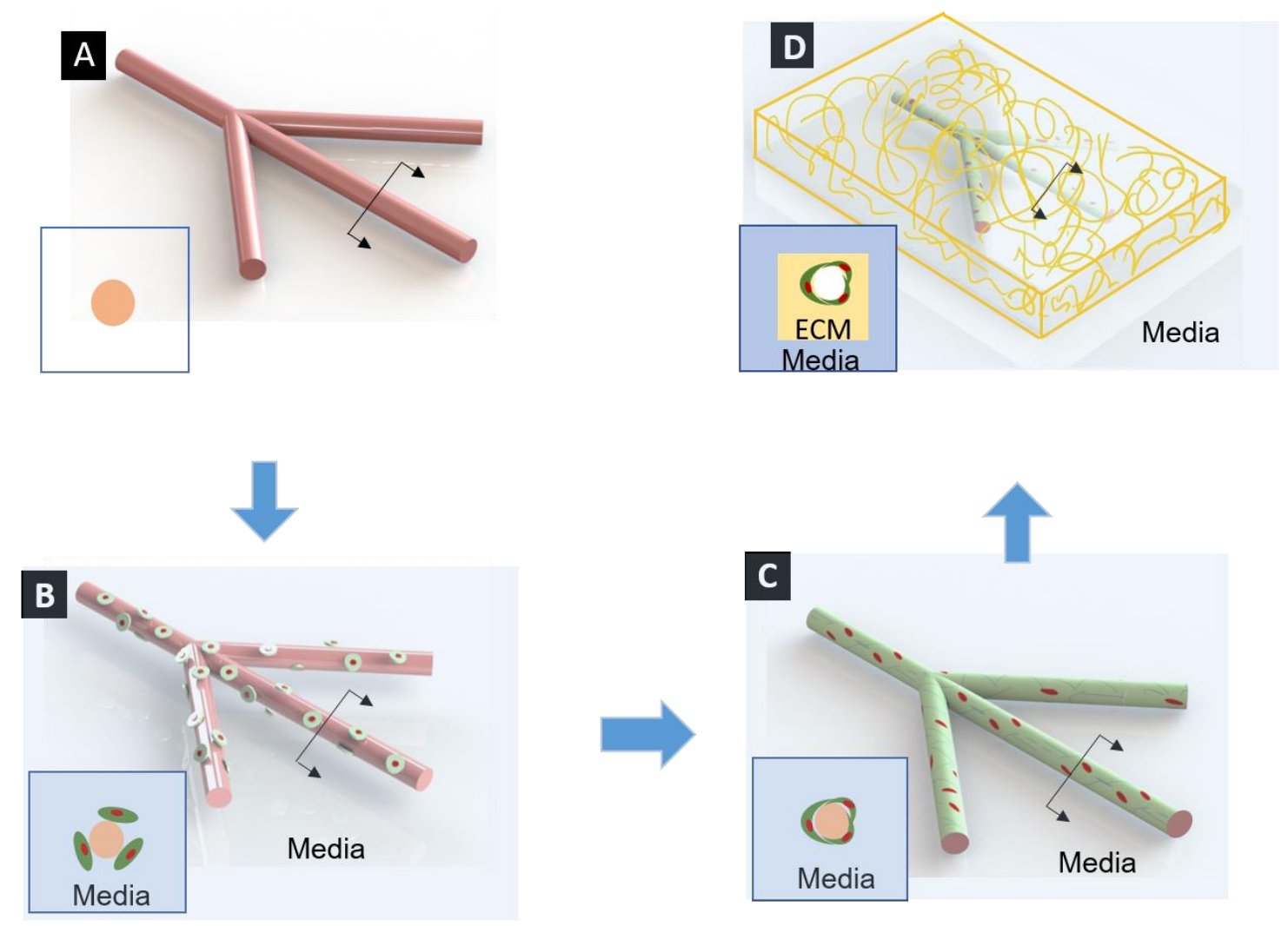

Figure 3. 1 Schematic illustrating of the concept of the formation of a microvascular network, and the cross-sectional images are inserted. A) Direct-write branched structure scaffold, B) seeded HDMECs on the surface of the scaffold in the media, C) HDMECs grew along the axis and around the circumference of the scaffold in the media, and D) vascularized-sheet with HDMECs-scaffold embedded in the ECM 


\subsection{Instrumentation and Equipment}

\subsubsection{3-Axis Robotic Dispensing System}

The biopolymer micro-fibers were direct-written from an integrated 3-Axis robotic dispensing system (Nordson Corporation, Westlake, OH; Figure 3.2A-B). This system is composed by the following subsystems: (1) JR 2203N 3-Axis Desktop Robot with accessory JR C-Points software (Janome Sewing Machine Co., Tokyo, Japan). The X-Y-Z robot axis has an operational range of 200 by 200 by 50 millimeters, respectively, with a positioning resolution of $0.005 \mathrm{~mm}$ for $\mathrm{X}$ - and Y-Axes and $0.0025 \mathrm{~mm}$ for the Z-Axis. The $\mathrm{X}$ - and $\mathrm{Y}$ - Axes can be operated from 7 to $500 \mathrm{~mm} / \mathrm{second}$, and the Z-Axis can be operated from 2.5 to $250 \mathrm{~mm} / \mathrm{second}$; (2) Valvemate 7100 Dispensing Valve Controller (Nordson Corporation, Westlake, $\mathrm{OH})$. It can adjust the valve open time from 0.001 to 99.9 seconds with 0.001 second increment by programming; (3) EFD-741 MD Series MicroDot Dispense Valve (Nordson Corporation, Westlake, OH); (4) EFD Optimum Dispensing Tips (Nordson Corporation, Westlake, $\mathrm{OH}$ ), the inner diameters are coded by hub colors (Red: $0.25 \mathrm{~mm}$, Clear: $0.20 \mathrm{~mm}$, Lavender: $0.15 \mathrm{~mm}$, Yellow: $0.10 \mathrm{~mm}$ ) (Figure 3.2C); (5) USB Digital Microscope Camera (Microview, Guangzhou, China). (6) 2F500-1W Feedback control heater (Cadet, Vancouver, WA); and (7) Customized Polycarbonate Thermal Enclosure. The heater and enclosure can be used to maintain a constant temperature and consistent solvent evaporation rate. 

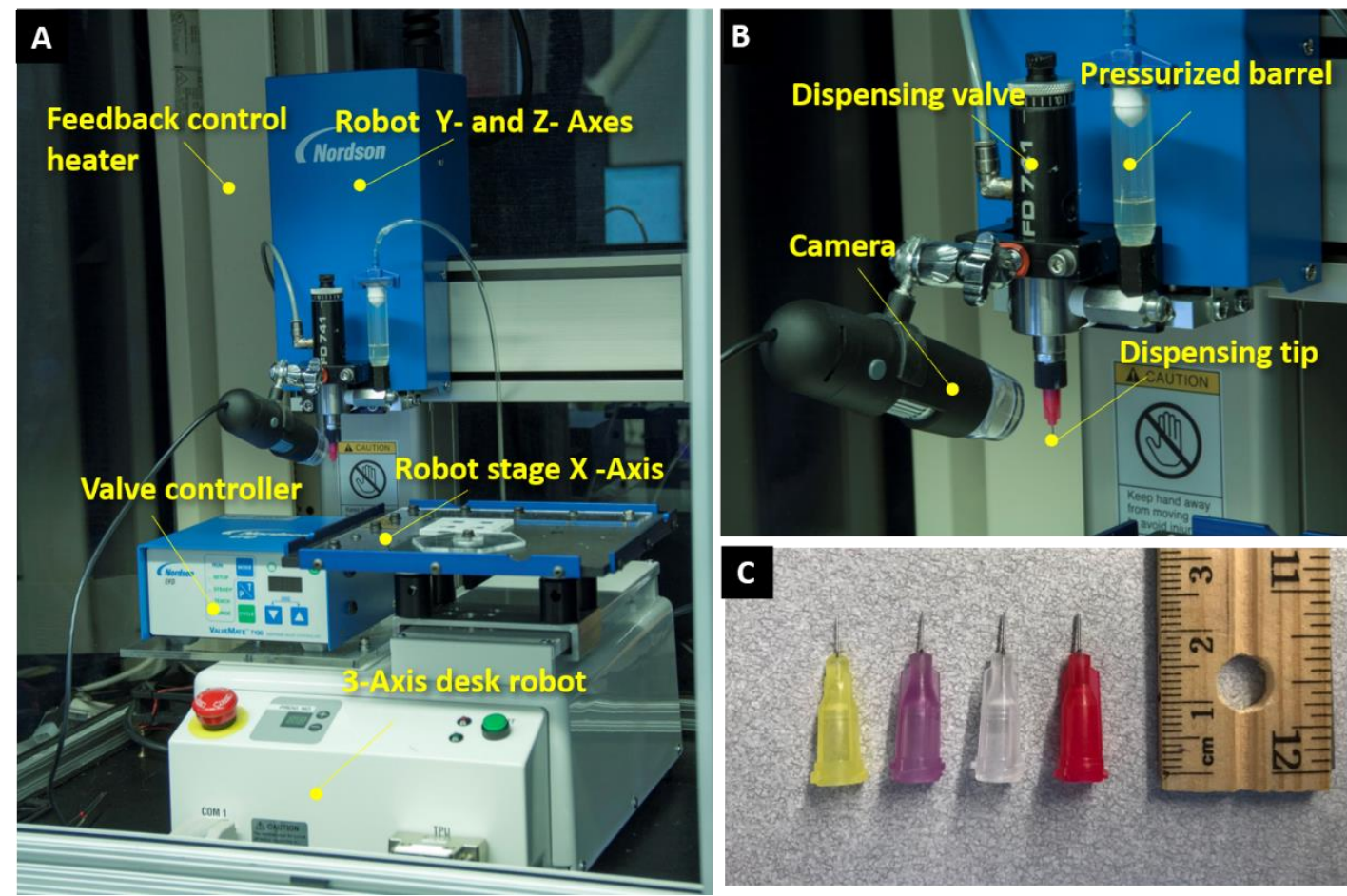

Figure 3. 2 Optical Images of 3-Axis robotic dispensing systems: A) Image of 3Axis robotic dispensing system housed inside an enclosure; B) Close up image of the dispensing valve with attached USB microscope for visualization; C) Image of EFD Optimum Dispensing Tips with four colors indicating four different inner diameters.

\subsubsection{Operation}

Turn on the computer, desk robot, and valve controller and adjust the air pressure to 15 psi. Launch the JR C-Points software to initialize the X-Y-Z Axes stage and then firmly mount the designed substrate on the X-Axis stage. The microscope camera was activated and focused on the needle, and the dispensing tip was manually positioned to the predefined initiation spot and while the Z-height was adjusted to find the optimum initial distance above the substrate by using "JOG" function (Figure 3.3A). Process parameters such as the initiation and 
termination $X-Y$ coordinates of the fiber, dispensing tip lift height, dispensing tip travel velocity (feed rate), and dispense time were input into the JR C-Points software (Figure 3.3B) and then uploaded into the memory of 3-Axis robotic dispensing system by clicking "Sent C\&T Data" button (Figure 3.3C) The Valve open time was manually input into the valve controller. After $3 \mathrm{~mL}$ of biopolymer solution is loaded into the pressurized barrel and purged to eliminate the trapped air, click the "Test Run" button (Figure 3.3C) to execute the automatic drawing process.

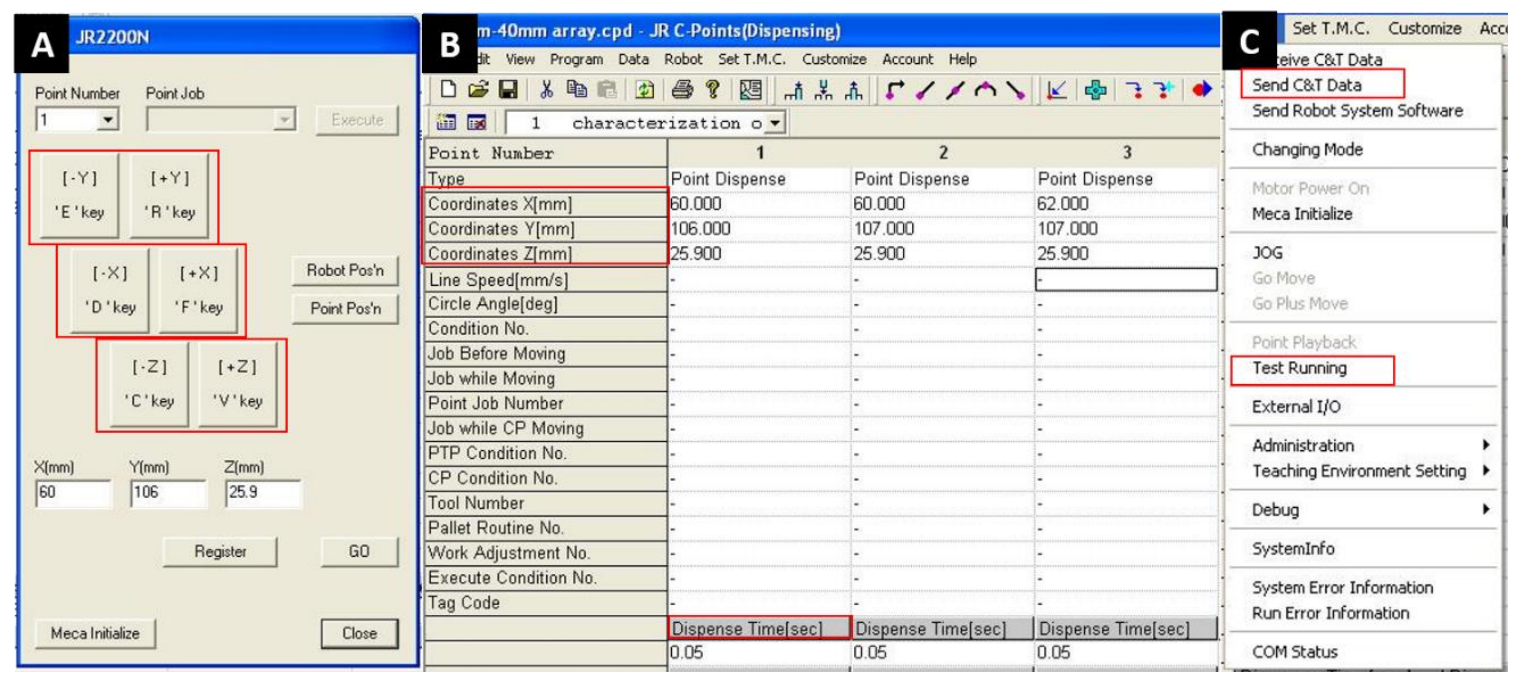

Figure 3. 3 Graphic interface of JR C-Points software

\subsubsection{Cleaning and maintenance}

Leaving the polymer in the dispense valve overnight may cause congestion, thus a proper cleaning and maintenance process at the end of the day or before changing different polymer solutions is critical. The pressurized barrel and dispensing tips can be simply discarded. To thoroughly clean the polymer solution, the EFD-741 Dispense Valve was carefully disassembled into components by 
following the manufacturer's guidance (Figure 3.4). Firstly, all components were washed by running hot water $\left(70^{\circ} \mathrm{C}\right)$ through the components to remove large polymer solution residue. Then all parts except the rubber O-ring were immersed into a beaker of acetone and subjected to an ultrasonic bath for 30 minutes. Subsequently, deionized (DI) water was used to rinse all the parts and the parts were then dried by compressed air. Needle packing assembly and piston/needle assembly needed to be lubricated with Nye Lubricant \#865 gel before being reinstalled.

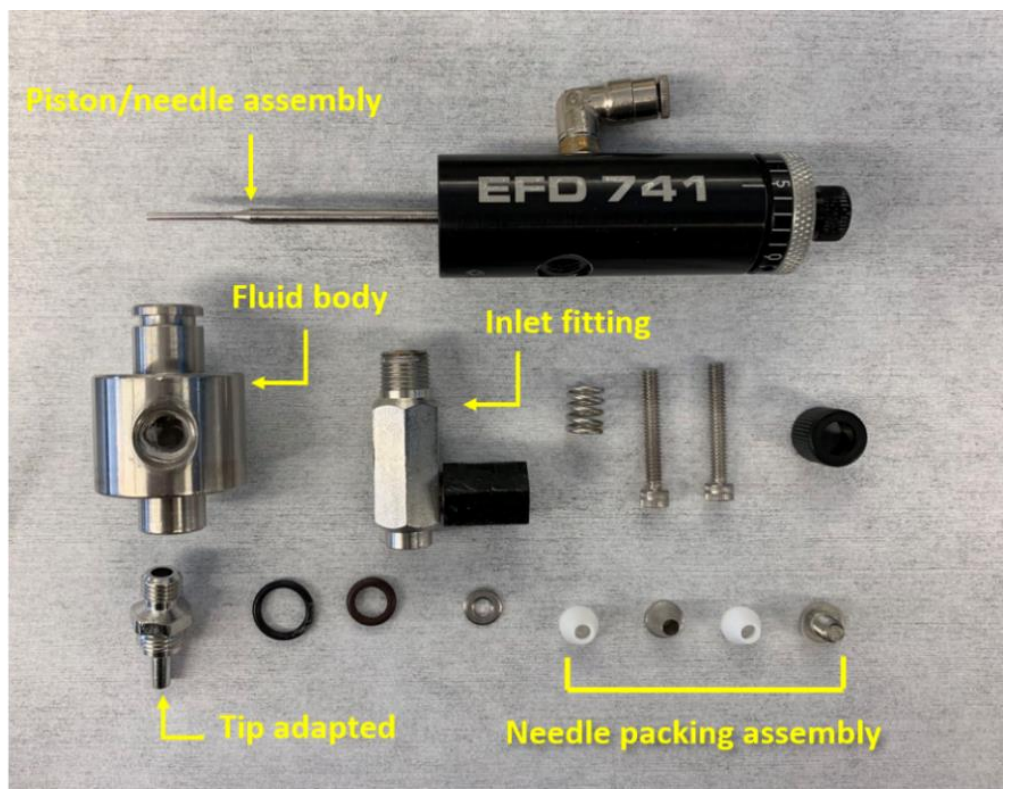

Figure 3. 4 Image of disassembling components of EFD 741 dispense valve

\subsubsection{Rheometer}

The viscosity of the biopolymer solutions was measured by rheometer (Physica MCR 300, Anton Paar, Austria; Figure 3.5). The principle of this rheometer is similar to a standard cone and plate viscometer, which applies shear 
on a fluid between the rotating cone and the static plateto measure the rotational resistance exerted by the fluid. The viscosity is calculated by the software from the resistance torque. This rheometer can determine a viscosity curve over a broad range of shear rates. A column-shaped heated/refrigerated water bath and circulator was attached and employed to maintain the test temperature.

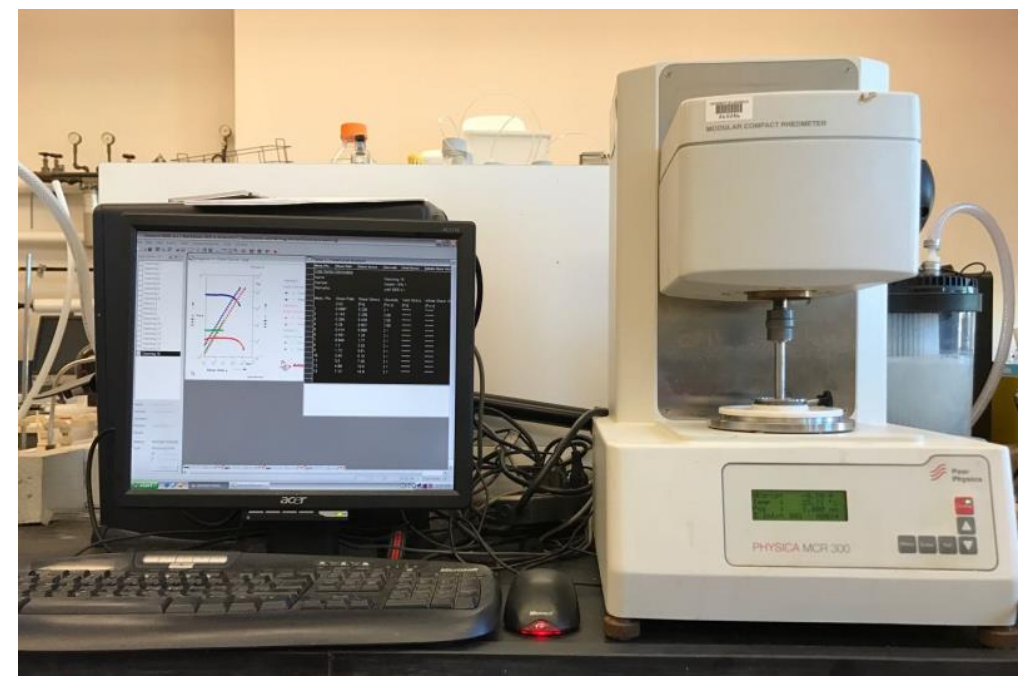

Figure 3. 5 Image of Physica MCR 300 rheometer under testing

Turn on the water bath and warm up the rheometer for least 15 minutes. Next, remove the cone and plate (the choosing cone is DG 26.7 with a diameter $=1 \mathrm{~mm}$ and plate is TEZ $150 \mathrm{P}-\mathrm{C}$ ) from the protect box and add $10 \mathrm{~mL}$ biopolymer solution into the plate. Then, properly mount the cone and plate to the position. Launch the Physica RheoPlus software and input the sample name, test temperature $\left(25^{\circ} \mathrm{C}\right)$, cone and plate code, shear rate range $(0.1-10001 / \mathrm{s})$ and time interval (150 s). By clicking the "Start" button, the machine will automatically operate by first heating to the desired temperature and then increasing the cone rotation speed until it reached the maximum rotational resistance was reached. 
The viscosity curve over different shear rate was plotted and saved to the computer. The rheometer was calibrated by the calibration standard fluid 5000 every month (Viscosity $=4930 \mathrm{mPa}{ }^{*} \mathrm{~S}$ at $25^{\circ} \mathrm{C}$, Brookfield, Middleboro, MA; Figure 3.6).

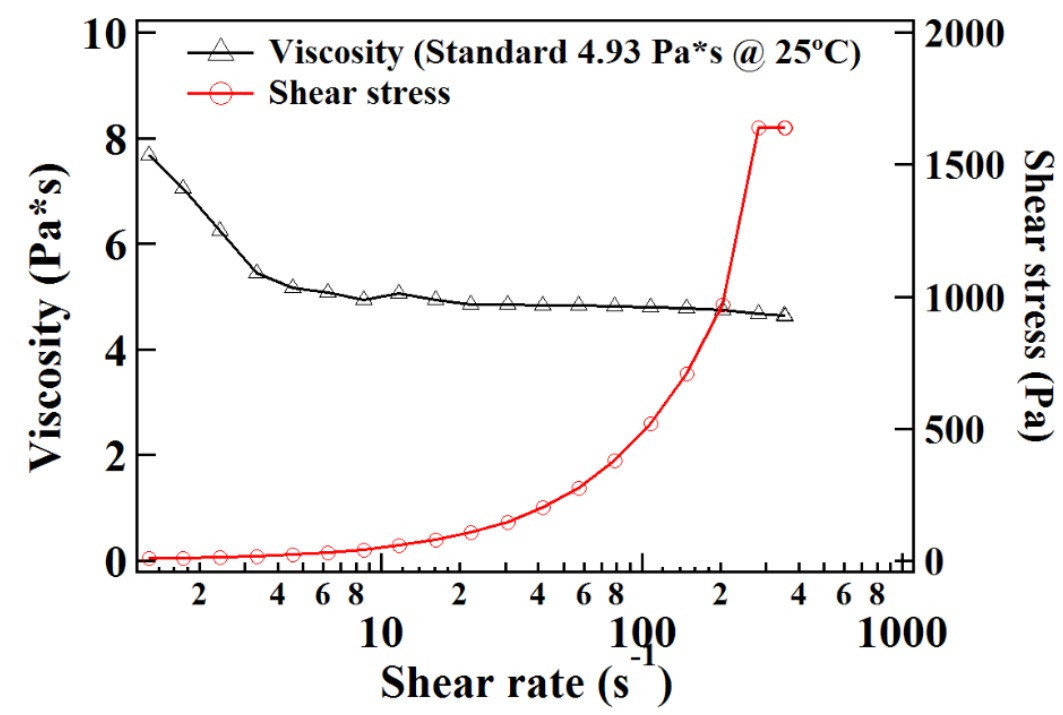

Figure 3. 6 The viscosity of standard fluid for calibration

\subsubsection{Du Nüoy-Paddy Surface Tension Measurement System}

The surface tension of the biopolymer solutions was measured by using the Du Nüoy-Paddy method. This method involves slowly lifting a rod from the surface of the solution. The force required to raise the rod from the solution's surface is measured and related to the solution's surface tension. The custom surface tension measurement system is shown in Figure 3.7. A glass rod (diameter $=3.2$ $\mathrm{mm}$ ) was vertically attached to a linear actuator (Firgelli L12) which was fixed to height adjusted stand. A precise balance (Mettler, PM100) was positioned to make sure the glass rod is vertical to the center of the balance weighing platform. A 
LabView VI was programmed to control the linear actuator expense and contract through an Arduino microcontroller (UNO). Two USB- cameras were also used to monitor and record the balance reading and the interface between the glass rod and the solution surface.

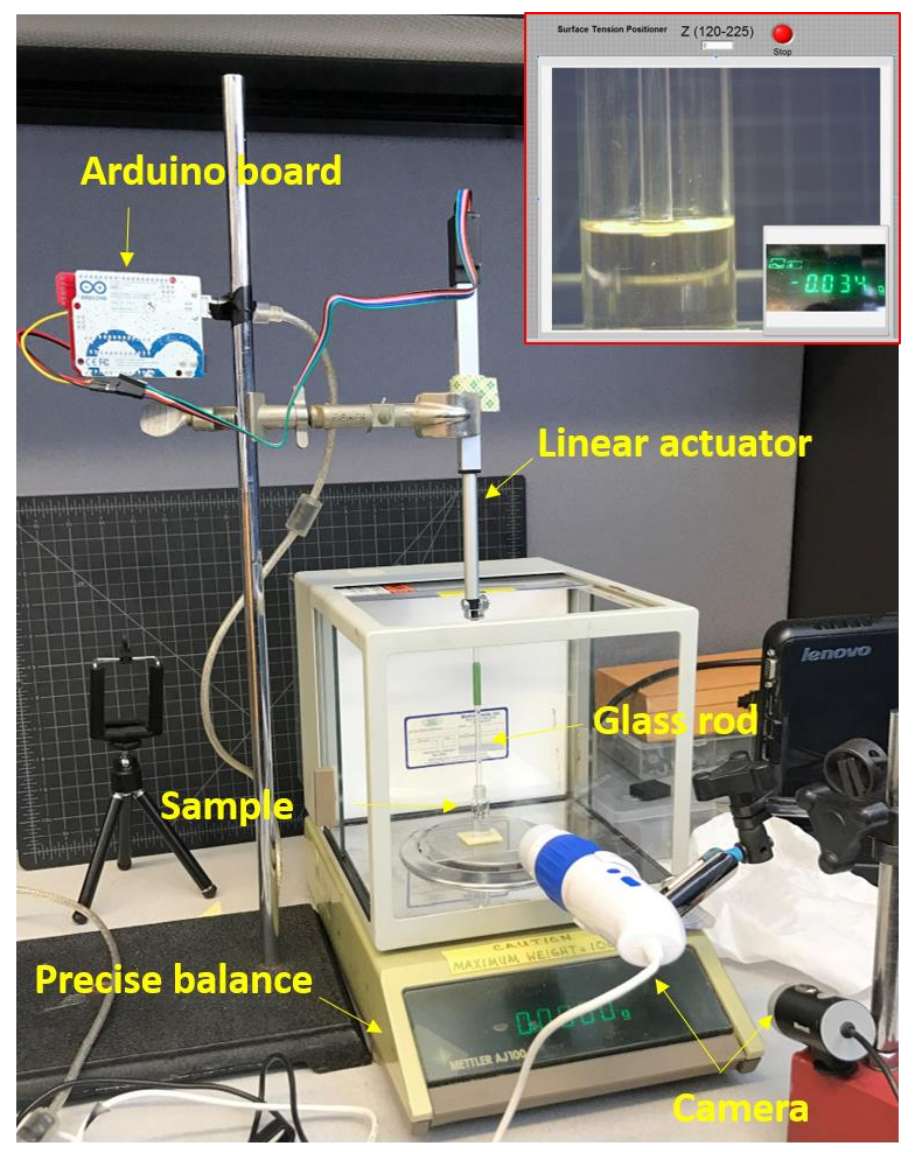

Figure 3. 7 Image of custom Du Nüoy-Paddy surface tension measurement system. Inserted images showed the instant images of the balance reading and the interface between the glass rod and the surface of the solution

A calibration was performed to make sure the LabView program and the Arduino microcontroller can control the linear actuator to expand and contract smoothly and accurately (Figure 3.8). $0.5 \mathrm{~mL}$ biopolymer solution was loaded into 
a small glass sample bottle and positioned on the center of the balance weighting platform. The glass rod was dipped into biopolymer solution a few millimeters under the surface and the balance reading $\left(\mathrm{M}_{1}\right)$ was recorded. The LabView program was employed to contract the linear actuator and record the balance reading $\left(M_{2}\right)$ when the glass rod was detached from the solution. The force $(F)$ exerted on the rod due to the surface tension was calculated by:

$$
F=\left(M_{1}-M_{2}\right) * g
$$

The surface tension, $\sigma$, could be calculated by:

$$
\sigma=\frac{F}{p * \cos (\theta)}
$$

Where the $p$ is the perimeter of the rod $(p=10.1 \mathrm{~mm}$ according to $d=3.2 \mathrm{~mm}), \theta$ is the contact angle of the solution on the rod. The glass rod was chosen since all tested solutions were found to wet glass completely such that $\theta$ approached 0 .

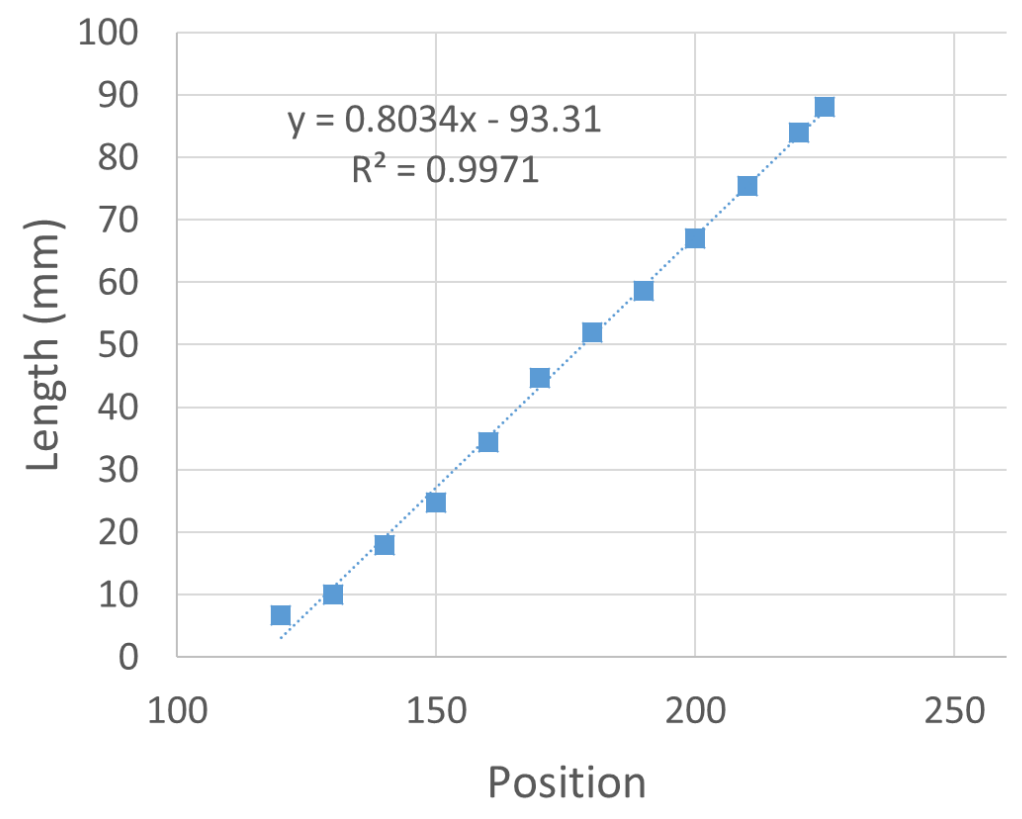


Figure 3. 8 Calibration of the linear actuator length corresponding the LabView Control position input

\subsubsection{Thermogravimetric Analyzer (TGA)}

Mass transfer coefficients of polymer solutions were measured by a thermogravimetric analyzer (SDT Q600 Simultaneous TGA/DSC, TA Instruments, New Castle, DE; Figure 3.9). The device can be used to measure high-precision weight change in the isothermal environment.

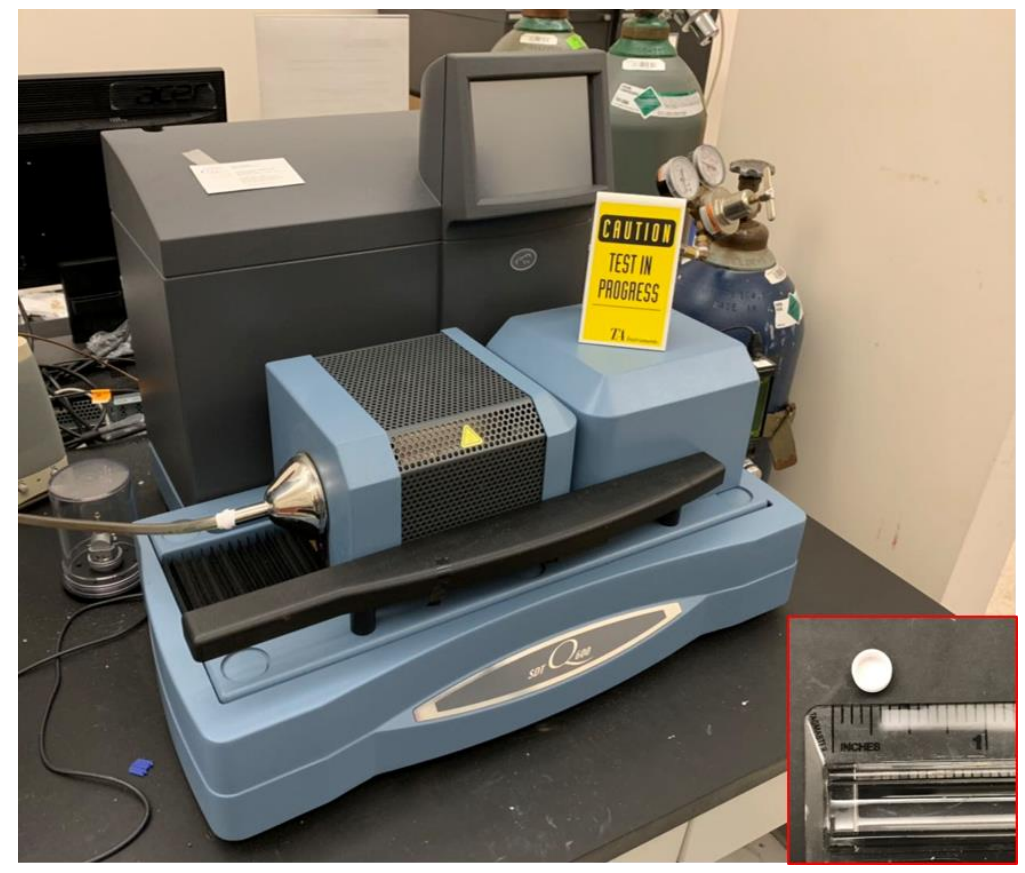

Figure 3. 9 Image of TA SDT Q600 Simultaneous TGA/DSC. Inserted image shows the ceramic cup that holds the sample

Turn on the machine and warm it up for 30 minutes. $\sim 30 \mathrm{mg}$ biopolymer solution was loaded into a ceramic cup after taring the empty cup. Launch the 
QSeires software (Figure 3.10) and change the gas source to air. Set up the running sequences as below: (1) ramp $10.0^{\circ} \mathrm{C} / \mathrm{min}$ to $25^{\circ} \mathrm{C}$; (2) isothermal for 120 mins; (3) end of the cycle. Click "Run" button to start the sequences.

The mass transfer coefficient, $x$, were then calculated by:

$$
\chi=\frac{-m_{(t)}}{A * c_{(t)}}
$$

where $m_{(t)}$ is the mass of the solution, $A$ is the area of the solution/air interface (A 34.5 $\mathrm{mm}^{2}$ for standard TGA ceramic cup), and $\mathrm{C}_{(\mathrm{t})}$ is the concentration of the solution determined from the below equation:

$$
c_{(t)}=\frac{m_{(t)}-m_{(\text {polymer })}}{m_{(t)} / \rho}
$$

Where $\mathrm{m}_{\text {(polymer) }}$ is the mass of polymer in the solution and $\rho$ is the density of the solution.

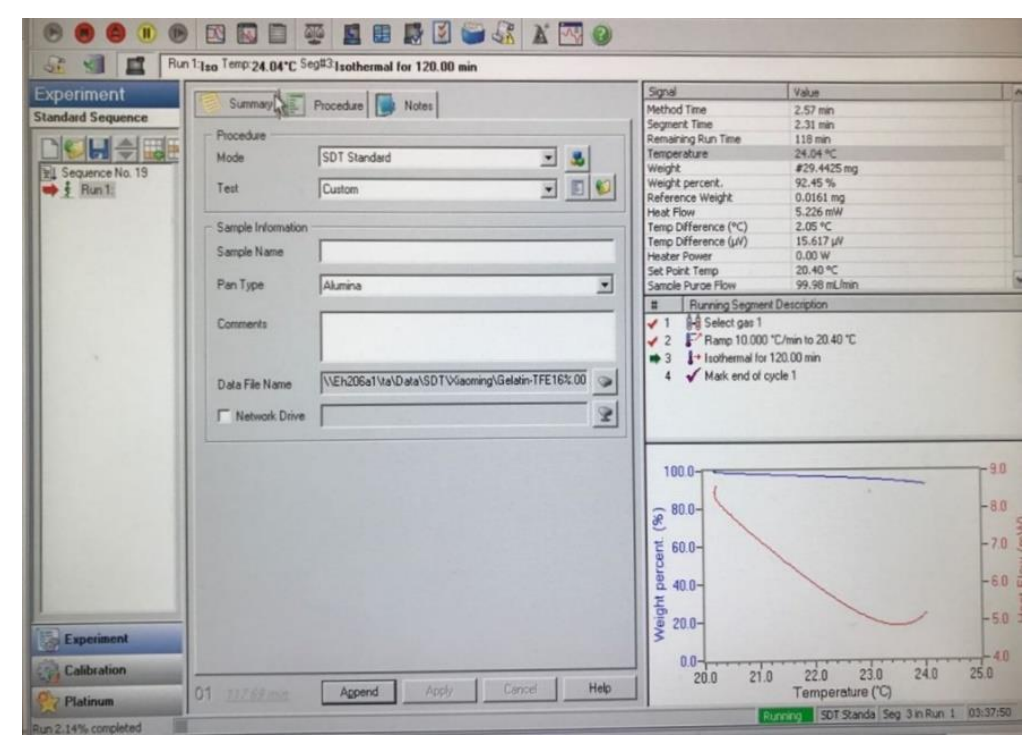

Figure 3. 10 Image of QSeries software graphics interface under testing 


\subsubsection{Fourier Transform Infrared Spectrometer (FT-IR)}

Polymer composites and copolymers were characterized by Spectrum 100 Fourier Transform Infrared Spectrometer (PerkinEImer, Shelton, CT; Figure 3.11). The physical properties of polymer composites and copolymer are affected by the structure of the molecular chains. When infrared radiation passes through a polymer sample, some of the radiation is transmitted while some radiation is absorbed by the sample. The resulting spectrum of absorbed light represents a distinctive "fingerprint" of the molecular structure of the sample and reveals the information of intermolecular hydrogen bonding.

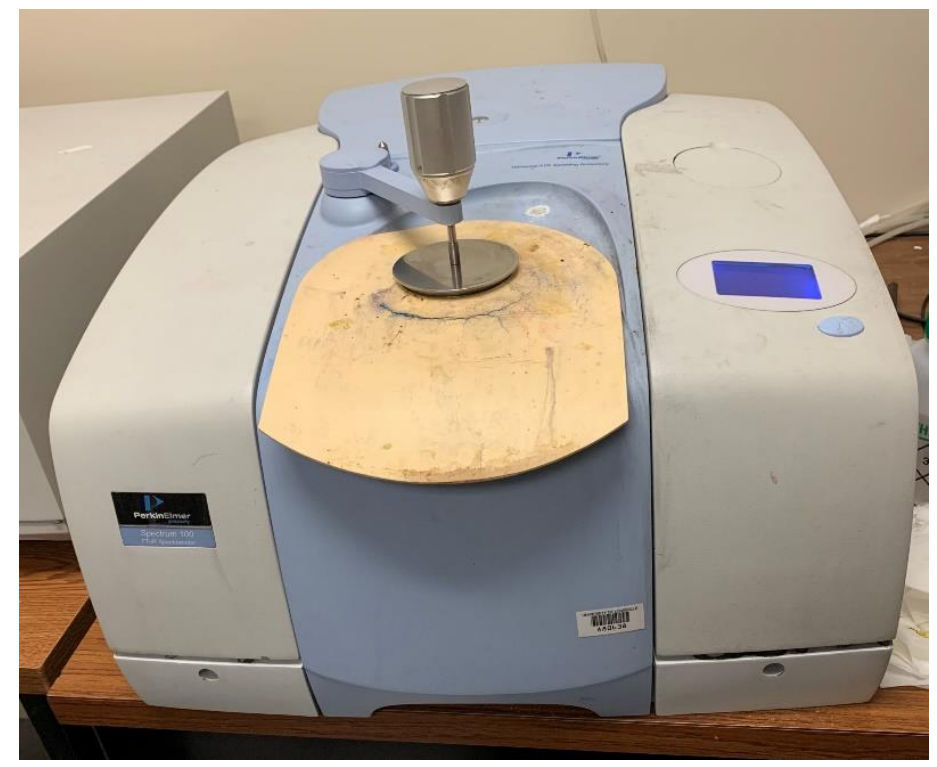

Figure 3. 11 Image of PerkinElmer Spectrum 100 FT-IR spectrometer

Sample films were prepared by casting $200 \mu \mathrm{L}$ of $6 \%$ solution of each polymer composites and copolymer in 2, 2, 2-Trifluoroethanol and chloroform, respectively. Films were heated to $60^{\circ} \mathrm{C}$ for 30 minutes and then left overnight at 
room temperature to remove residual solvent. A background run is required to eliminate the $\mathrm{H}_{2} \mathrm{O}$ and $\mathrm{CO}_{2}$ band in the air. Unless otherwise noted, the spectrums were obtained with 32 scans per sample ranging from $4000 \mathrm{~cm}^{-1}$ to $650 \mathrm{~cm}^{-1}$ (corresponding to wavelength from 2,500 nm to $15,000 \mathrm{~nm}$ ).

\subsubsection{Nuclear Magnetic Resonance (NMR) Spectroscopy}

Copolymers were characterized by Varian Inova $400 \mathrm{MHz}$ NMR (Agilent Technologies, Santa Clara, CA; Figure 3.12). The NMR study could identify the monomers' ratios and the formation of new bonds.

The target copolymer sample $(20 \mathrm{mg})$ was dissolved in $500 \mu \mathrm{L} \mathrm{CDCl} 3$ in an NMR glass tube to form a slurry, which was then further sonicated to form a clear solution. The ejection, insertion, spinning, locking, and shimming were all controlled by VnmrJ Software. All ${ }^{1}$ HNMR spectra were acquired at frequencies of 399.8 $\mathrm{MHz}$ and conducted at $25^{\circ} \mathrm{C}$. The chemical shifts are reported in $\delta(\mathrm{ppm})$ values relative to $\mathrm{CDCl}_{3}\left(\delta=7.26 \mathrm{ppm}\right.$ for $\left.{ }^{1} \mathrm{HNMR}\right)$. 


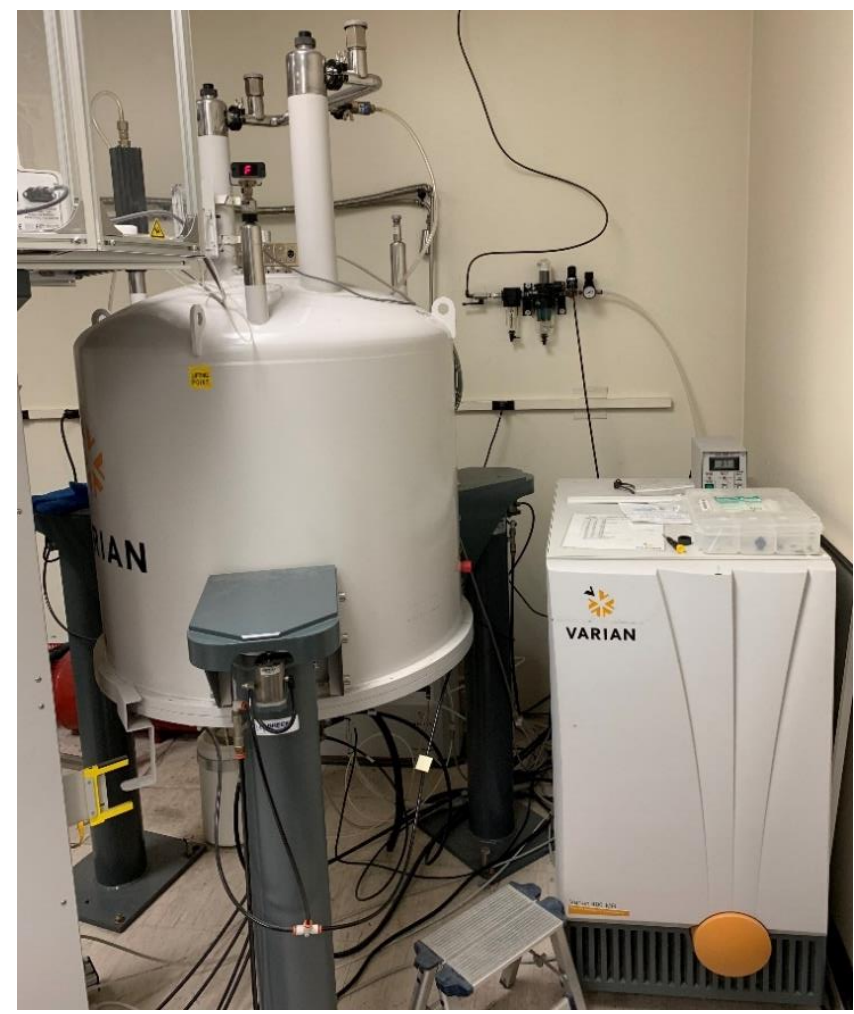

Figure 3. 12 Image of Varian Inova $400 \mathrm{MHz}$ NMR

\subsubsection{Scanning Electron Microscope (SEM)}

The LEO Supra 35 VP field-emission variable pressure scanning electron microscope (VPSEM) (Carl Zeiss, Oberochan, Germany; Figure 3.13A) and VEGA 3 tungsten thermionic emission scanning electron microscope (TESCAN, Brno, Czechia; Figure 3.13B) were both used to image and measure the biopolymer fibers and observe the cell grow on the scaffold and in the extracellular matrix. Despite the fact that the two SEMs have different electron sources, they both work in a vacuum environment $\left(10^{-4}-10^{-5} \mathrm{~Pa}\right)$ and have "Secondary Beam" detector. While the focused electron beam scans the sample, the secondary 
electrons are collected and converted to represent topographical imagery on the screen.

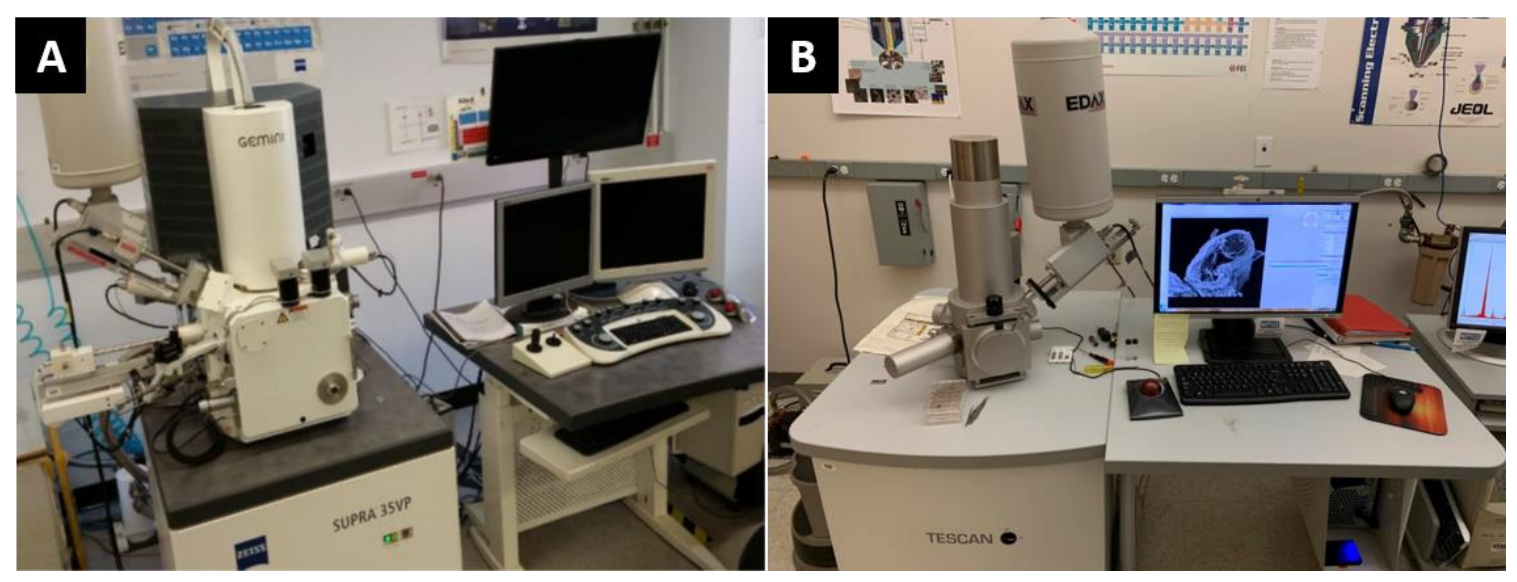

Figure 3. 13 Image of A) Carl Zeiss Supra 35 VP field-emission SEM. B) TESCAN VEGA 3 tungsten thermionic emission SEM

For a non-conductive sample, electron accumulation within the sample, or "charging," often occurs, resulting in sample damage and reduced image quality. Before the samples were loaded into the SEM chamber, they were all sputter coated with a uniform thickness thin film of gold-palladium by the benchtop SPI sputtering machine (Structure Probe, Inc., West Chester, PA; Figure 3.14) to improve conductivity. The sample was first placed in a chamber filled with Argon and then the chamber was vacuumed to $\sim 80$ millitorr. A visible discharge will be observed when the plasma current was set to $\sim 18$ milliampere. To deposit a 10 nm-thick gold-palladium film, the sputtering time was set to 90 seconds. 


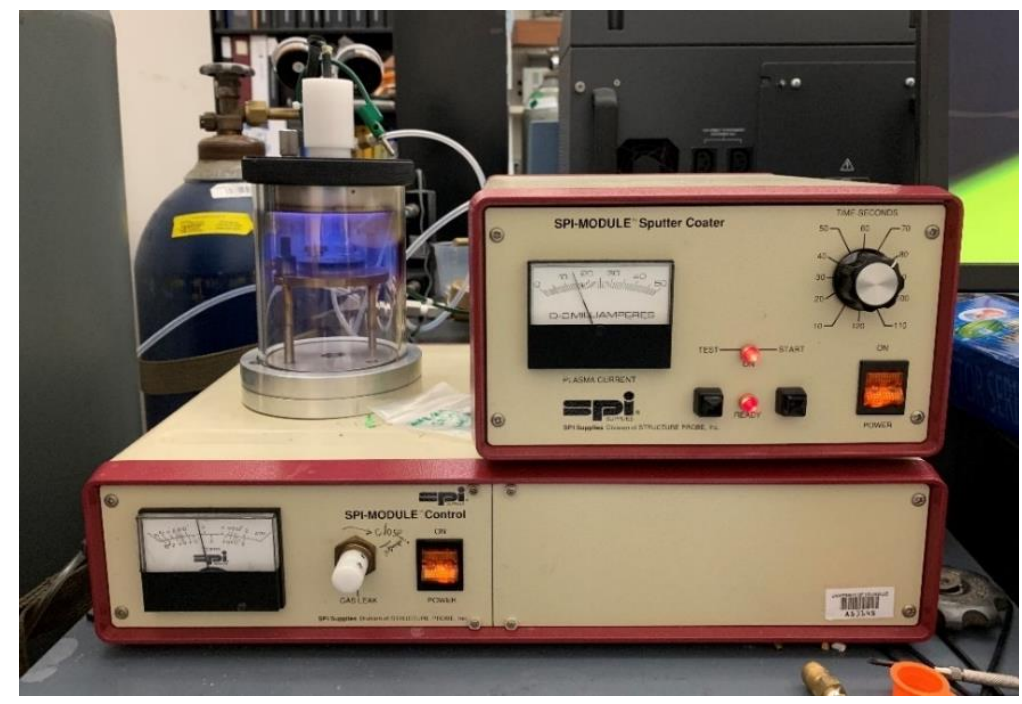

Figure 3. 14 Image of SPI-module sputter coater

The basic operations for these two SEMs are almost the same; however, the software is different and requires individual training for each. The sputter coated samples were attached to the desired stage platform with conductive carbon tape and loaded into the SEM chamber. Upon completion of the vacuum to reach the working air pressure, the working voltage and adjusted the working distance are manually selected before starting imaging. Unless otherwise noted, samples were observed with $2-10 \mathrm{KV}$ working voltage and the working distance of $8-12 \mathrm{~mm}$.

\subsubsection{Laser Cutting System}

Several custom acrylic frames used for fiber fabrication and cell culture were made by the LS1630 60-Watt $\mathrm{CO}_{2}$ laser cutting system (Boss Laser, LLC, Sanford, FL; Figure 3.15). The X-Y-Z axis stage has an operational volume of 75 by 40 by 25 centimeters, respectively, with a positioning resolution of 0.025 
millimeters along all three axes. A 250 cubic foot per minute (CFM) exhaust fan system is employed to vent the volatile fumes/particulates out of the laser cutting system enclosure and safely into the building HVAC fume extraction system.
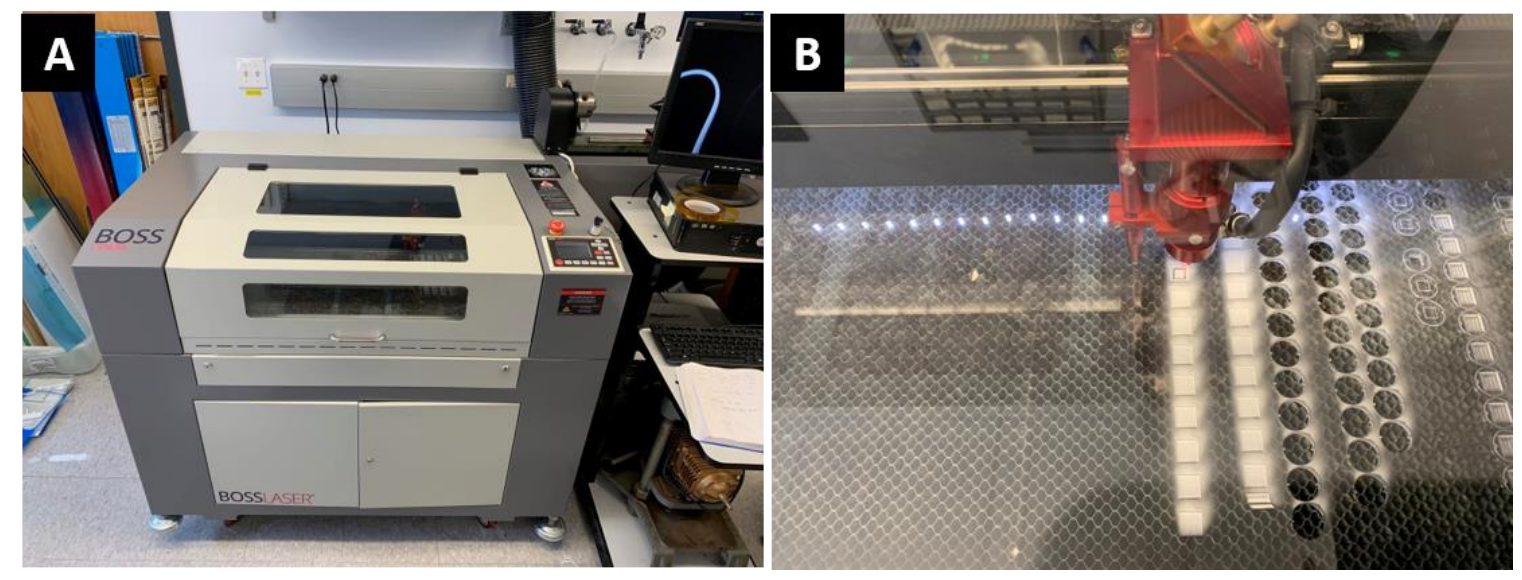

Figure 3. 15 Image of Boss LS1630 laser cutting system A) System body, B) Laser cut under the working

The desired structure of interest was designed by SolidWorks and converted the 3D part file into 2D Adobe Illustrator file (.AI). The .Al file is imported into the RDWorks software. The laser head is positioned to the desired location over material to be cut and adjusted the laser beam to focus on the top surface. Set cutting speed and laser power with the Work tab. A power setting of $50 \%$ and cutting speed of $10 \mathrm{~mm} / \mathrm{s}$ is utilized for cutting $3 \mathrm{~mm}$ thick acrylic. The cell culture frame with a recessed platform was achieved by two layers of operation (Figure 3.16). The first layer was to create the recessed platform by out of focus laser scan mode; the second layer was to finally cut through the open window and boundary by cut mode. The operation parameters are shown in Table 3.1. 


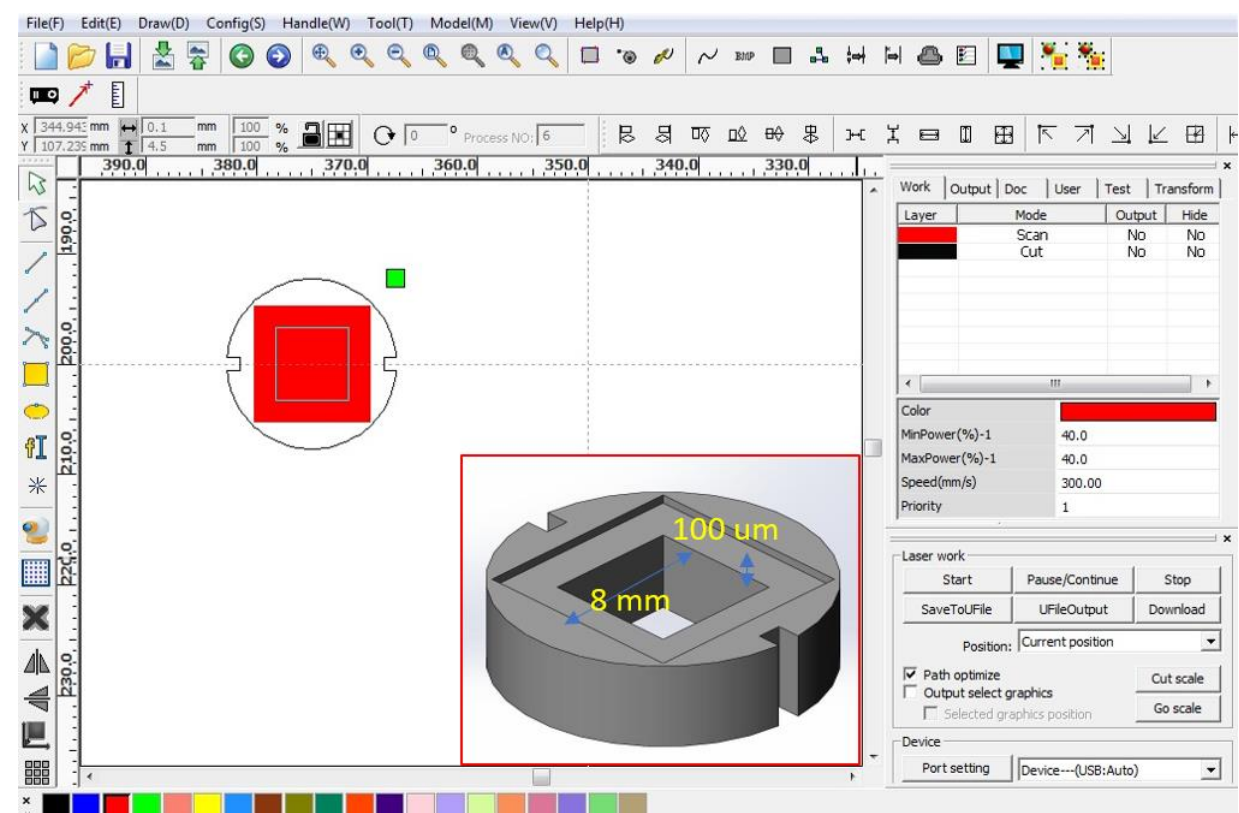

Figure 3. 16 Image of RDWorks software graphic interface with two layers of operations: Red (scan) and Black (cut). The insert image shows the 3D part file of the cell culture fiber frame with a recessed platform

Table 3. 1 The operation parameters for cell culture fiber frame with a recessed platform

\begin{tabular}{cccc}
\hline Laser mode & Focus (mm) & Speed (mm/s) & Power (\%) \\
\hline Scan & -10 & 300 & 40 \\
Cut & 0 & 10 & 50 \\
\hline
\end{tabular}

\subsubsection{Cell Culture Related Equipment}

Cell culture, subculture, and seeding were all performed in the Biomimetics \& Tissue Engineering Laboratory at the University of Louisville (directed by Dr. 
Patricia Soucy, BSL-2). The main aseptic area is supplied by 1300 Series A2 Laminar-Flow Hood (Thermo Scientific, Waltham, MA; Figure 3.17) connected with an aspiration pump and waste bottle. Cells were cryopreserved in VWR CryoPro liquid nitrogen tank (Figure 3.18). Fisher Scientific ISOTEMP 210 Water Bath (Set to $37^{\circ} \mathrm{C}$, Figure 3.19 ) was used to thaw the cells or prewarm a necessary solution. Cells were incubated in the VWR $\mathrm{CO}_{2}$ incubator (Figure 3.20) with controlled temperature $\left(37^{\circ} \mathrm{C}\right)$, high relative humidity $(\sim 60 \%)$ and elevated $\mathrm{CO}_{2}$ concentration $(5.0 \%)$.

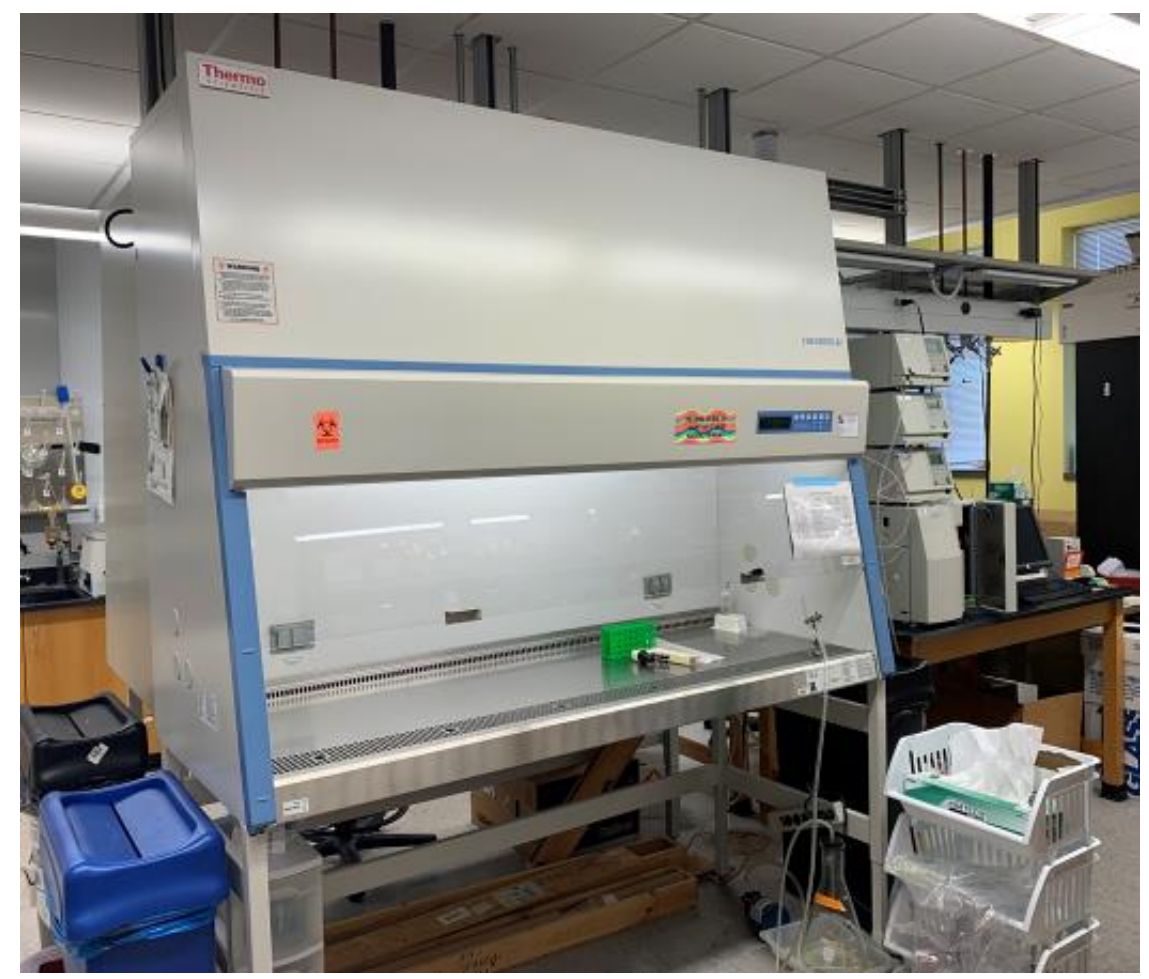

Figure 3. 17 Image of Thermo Scientific 1300 Series A2 Laminar-Flow Hood 


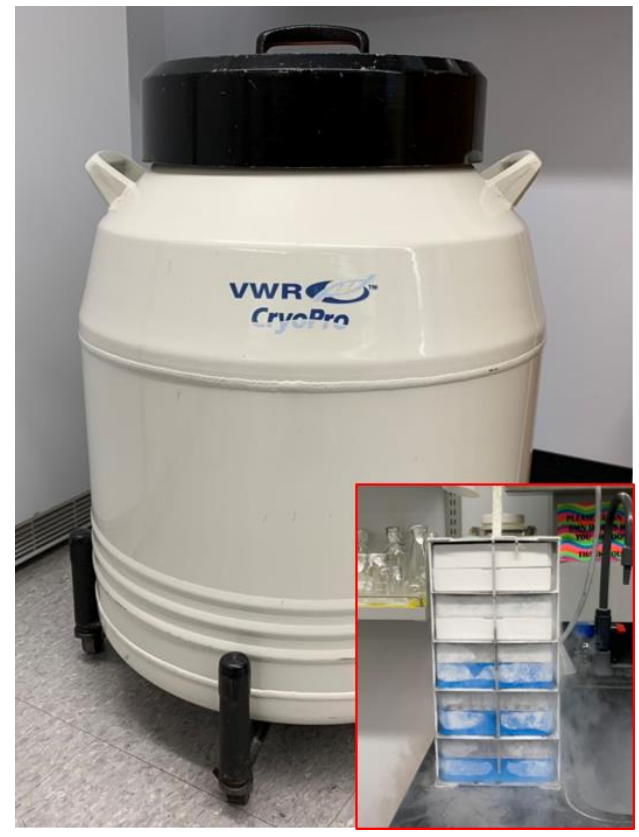

Figure 3. 18 Image of VWR CryoPro liquid nitrogen tank and the insert image shows the drawers in a rack system inside of the tank

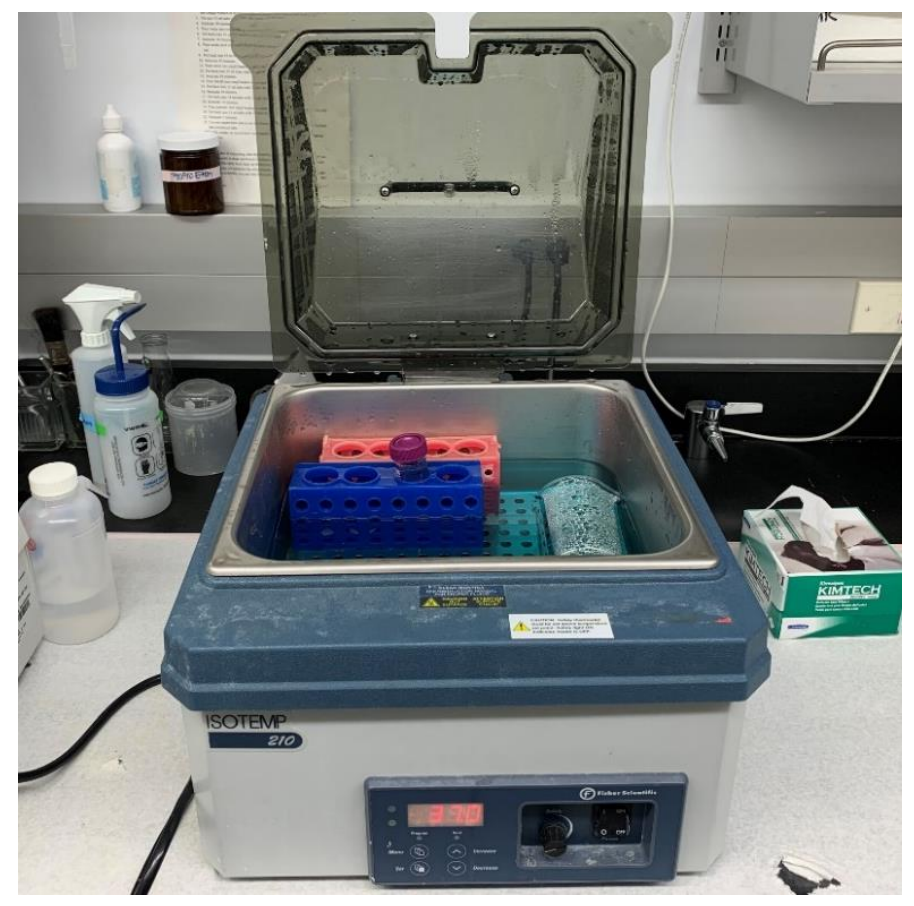

Figure 3. 19 Image of Fisher Scientific ISOTEMP 210 Water Bath 


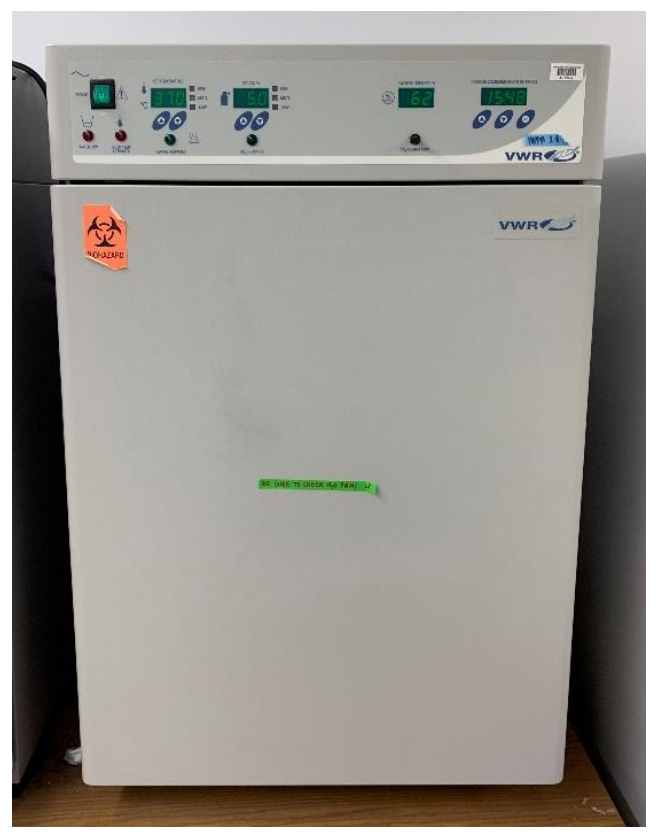

Figure 3. 20 Image of VWR $\mathrm{CO}_{2}$ incubator

While performing the cell subcultivation, the cell suspension was centrifuged by Allegra R Centrifuge (Beckman Coulter, Brea, CA; Figure 3.21) with GH-3.8 swinging bucket rotors at $220 \mathrm{G}$ (980 RPM) for 5 minutes to increase the concentration or to wash off the reagent.

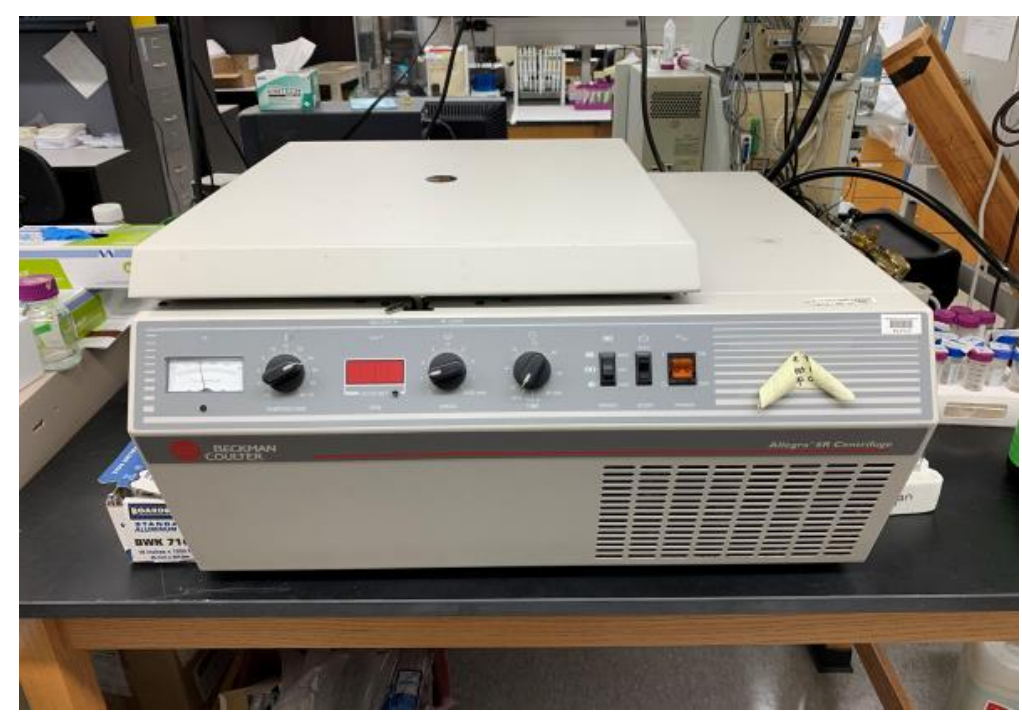

Figure 3. 21 Image of Beckman Coulter Allegra 6R Centrifuge 
Auto T4 Cellometer (Nexcelom Bioscience, Lawrence MA; Figure 3.22A) was utilized to determine the cell concentration and mean diameter of the cells to decide the seeding density. $20 \mu \mathrm{L}$ of cell suspension was pipetted into the compatible cellometer disposable counting chambers (Figure 3.22B) and inserted into the cellometer. Select the cell types and click "Count" to get results (Figure 3.22C).

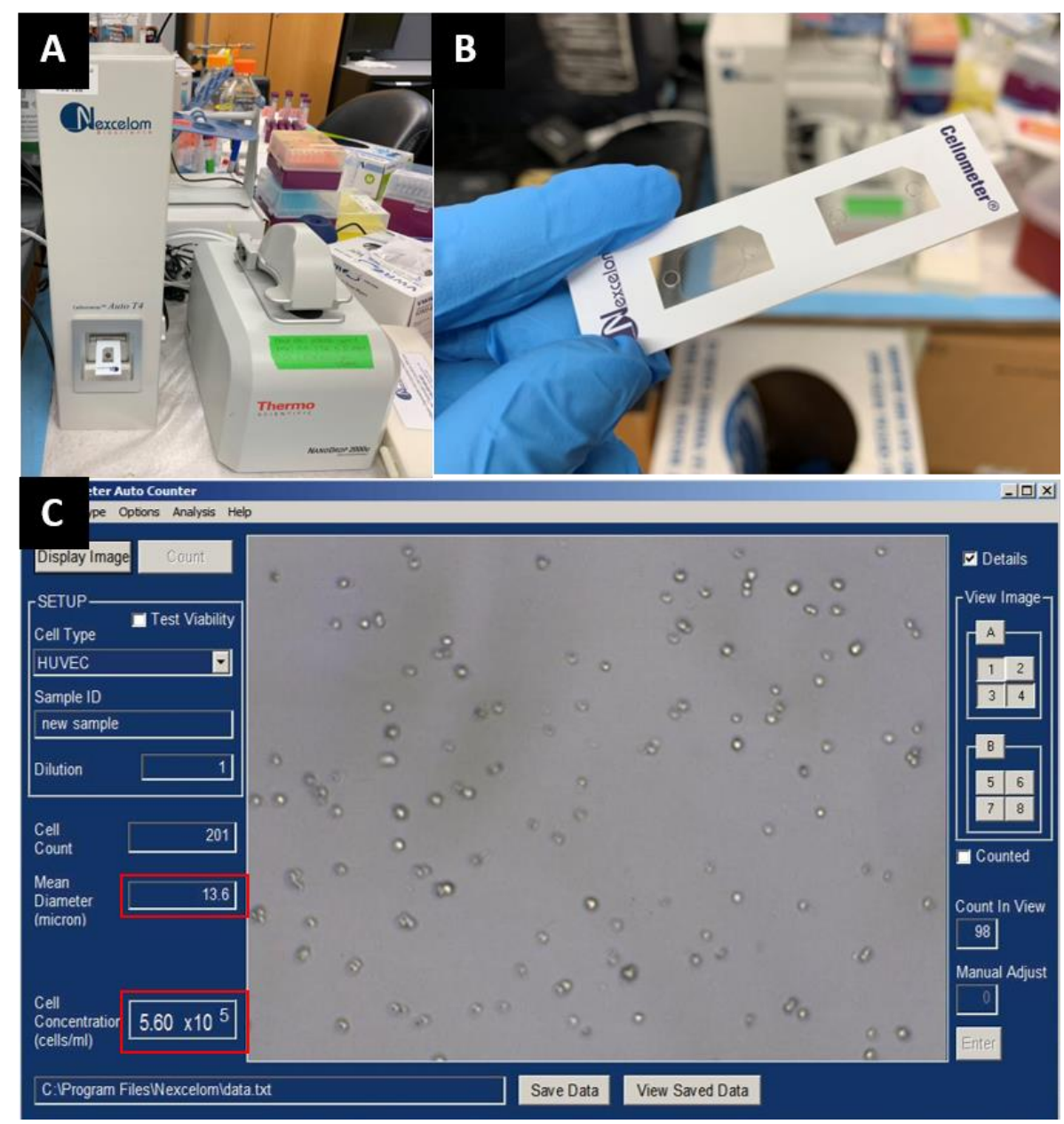

Figure 3. 22 Image of cell count: A) Nexcelom Auto T4 Cellometer, B) Nexcelom disposable counting chambers, and C) Accessory software graphic interface showed count results 


\subsubsection{Epi-fluorescence Microscope}

A TE2000-U inverted microscope (Nikon, Shinagawa, Japan; Figure 3.23) with an X-Cite 120 fluorescence illumination system (Excelitas Technologies, Waltham, MA) and QIMAGING Retiga 2000R CCD camera (Teledyne, Surrey, Canada) was used to captured fluorescence images. This fluorescence microscope system was installed with three filters (1) Blue filter shows FITC and Alexa 488 (green colors); (2) Green filter shows Alexa Fluor 566 and Rhodamine (red colors), and (3) Violet filter shows DAPI and Alexa Fluor 350 (blue color). Cell samples need to be fixed first and then stained with different dyes prior to taking images. 10X, 20X, and 40X objectives were all used for capturing different magnification images.

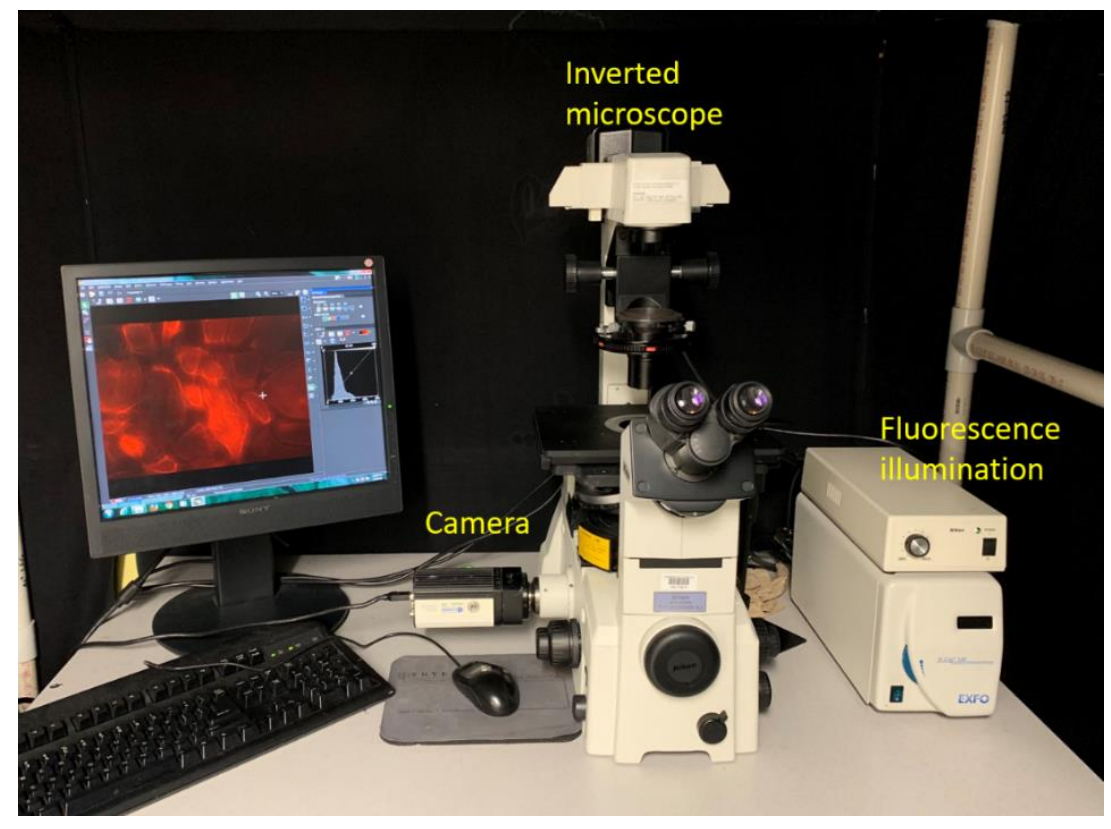

Figure 3. 23 Image of Nikon TE2000-U inverted microscope with an X-Cite 120 fluorescence illumination system and QIMAGING Retiga 2000R CCD camera 


\subsubsection{Confocal Microscope}

An Eclipse Ti- A1R confocal microscope (Nikon, Shinagawa, Japan; Figure 3.24) was employed to generate three-dimensional views of cells on the scaffold to evaluate if the cells could grow circumferentially around the fibers and if the neighboring cells can form cell-cell junctions. This confocal microscope system consisted of an inverted microscope and an A1R confocal laser system, which including a laser source, scanner controller, scan head, stage joystick, LED source, and a PC workstation. This confocal microscope system has three lasers excitation and corresponding filters: (1) $403.8 \mathrm{~nm}$ for DAPI (show blue color), (2) $487.8 \mathrm{~nm}$ for FITC (show green color), and (3) $561.6 \mathrm{~nm}$ for TxRed (show red color). 20X and 40X objective were used for different magnification images, and both objectives need to be immersed with oil. Accessory NIS Elements software could switch the objectives, select the different channels and filters, adjust the focal length and area, and change scanning speed. Unless otherwise noted, the pinhole was set to $1.5 \mathrm{AU}$, the laser power of 4 channels + TD were set to $5 \%$, gain (high voltage) was adjusted between 60 to 120 , and Offset was always set to 0 . All zstacks were taken with a $0.2 \mu \mathrm{m}$ step size. Similar to the fluorescence microscope, all the samples need to be fixed and stained with different dyes. However, due to the difficulty to transfer the scaffold onto standard microscope slides from a standard well plate, and the limited depth of field of objectives for suspended scaffolds, samples used for confocal imaging were all cultured and observed under two walls Chambered Cover Glass System (Cole-parmer, Vernon Hills, IL. Growth 
area $=4.0 \mathrm{~cm}^{2}$ and the working volume $=1.5 \mathrm{~mL}$; Figure 3.25$)$. The ultra-thin $(0.13$ $\mathrm{mm}$ ) cover glasses on the bottom permit viewing of the cells on the suspended scaffolds.

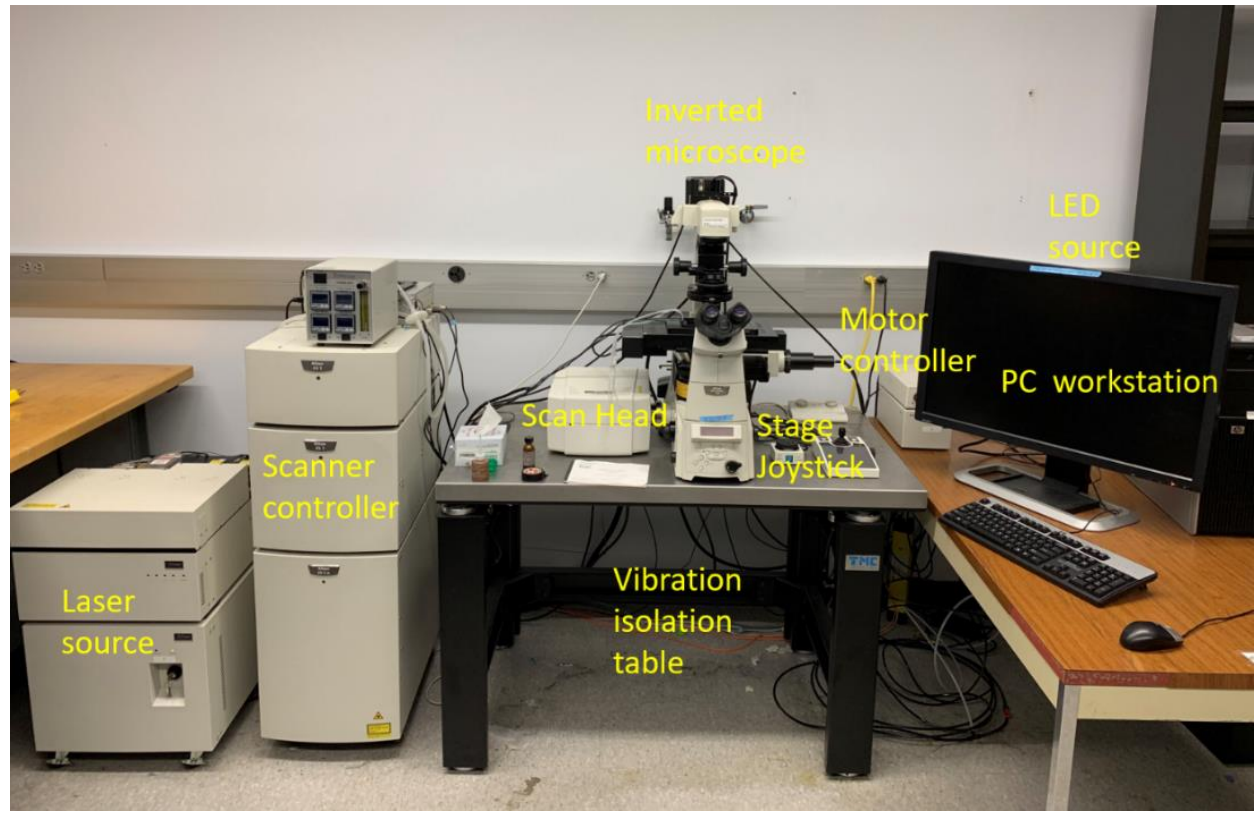

Figure 3. 24 Image of Nikon Eclipse Ti inverted microscope with A1R confocal laser system

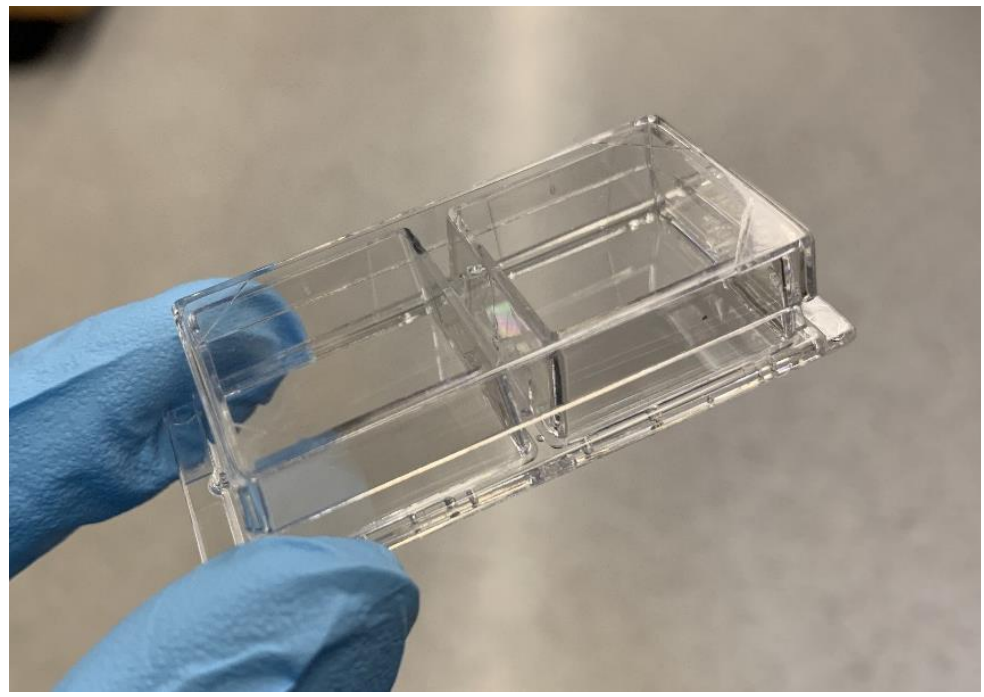

Figure 3. 25 Image of the two walls Chambered Cover Glass System 


\subsection{Methodology}

\subsubsection{Biopolymer Solution Preparation}

The solvent selection is critical to fabricating the biopolymer fiber successfully by using the direct-write technique. According to the previous research in our group, the ideal solvent should meet the following two criterions: (1) the capability of the solvent to dissolve the polymer within a range of concentrations sufficient for direct-write; (2) moderate volatility as defined by a boiling point ranging from 60 to $140^{\circ} \mathrm{C}$.

\subsubsection{Gelatin, PLGA, and Composites' Solution}

Although there are a variety of organic solvents including polar and nonpolar solvent that could successfully dissolve PLGA, gelatin is only dissolved in polar organic solvents such as 2,2,2-trifluoroethanol (TFE, BP $=78{ }^{\circ} \mathrm{C}$ ), 1,1,1,3,3,3-hexafluoro-2-propanol (HFIP, BP $=58.2^{\circ} \mathrm{C}$ ), and acetic acid (AA, BP $=$ $\left.118{ }^{\circ} \mathrm{C}\right)$ because it has strong polarity [196]. Thus, TFE and acetic acid were chosen to perform preliminary experiments to figure out the optimal solvent for direct-written micro-fibers. 


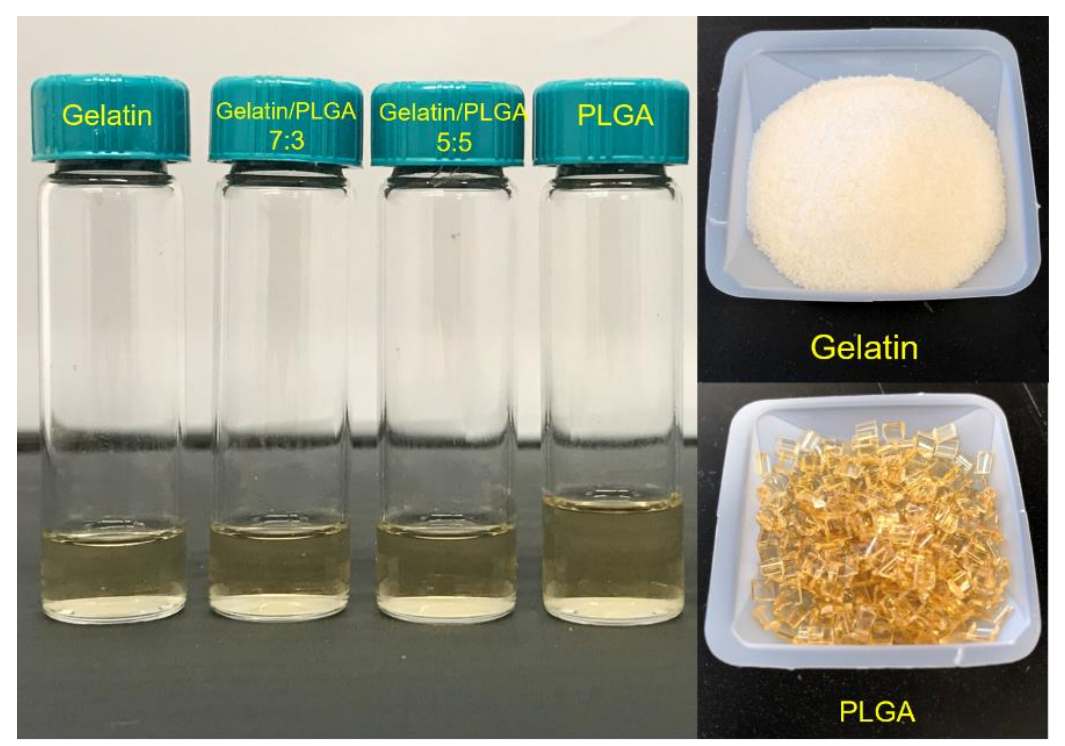

Figure 3. 26 Image of gelatin and PLGA and their composites solution in TFE

Gelatin Type A from porcine skin (gel strength 300), 2,2,2-trifluoroethanol, ReagentPlus $₫$ grade, $\geq 99 \%$ ), and acetic acid ( $\geq 99 \%)$ were all purchased from Sigma-Aldrich (St. Louis, MO). 50:50 DL-PLGA was purchased from Lactel Absorbable Polymers (Birmingham, AL). Gelatin solutions were produced by dissolving powder gelatin into AA or TFE; PLGA solutions were produced by dissolving granular PLGA in TFE; Gelatin/PLGA composite solutions were produced by physically mixing the gelatin and PLGA at a weight ratio of 70:30 and 50:50 into TFE and maintained the whole concentration. All the solutions were vortex stirred for 3 minutes for pre-mix and followed by magnetic stir for 6 hours at room temperature. All the solutions were confirmed complete dissolution by inspected homogeneity and optical clarity after stirring. The solution remained clear after sitting for one week. 


\subsubsection{PLA and PLA-PEG Copolymer's solution}

Polylactic acid (PLA, 3001D, melt flow index of $1.1 @ 210^{\circ} \mathrm{C}$ ) and Polyethylene glycol (PEG, molecular weight 1000) were kindly supplied by Dr. Kunal Kate. Chloroform ( $\geq 99.8 \%$ ) was purchased from Sigma-Aldrich (St. Louis, MO). PLA-PEG (50:50) and PLA-PEG (90:10) were previously synthesized by Dr. Kunal. PLA-PEG copolymers were synthesized by reacting the different ratio components (Table 3.2) in a three-neck glass reactor at $205^{\circ} \mathrm{C}$ with stirring under a nitrogen atmosphere for 4 hours. The copolymer synthesis setup is shown in Figure 3.27. The resulting copolymer was cooled at room temperature and stored under moisture-free conditions. A home blender was used to ground the copolymer into a powder for solution preparation. 


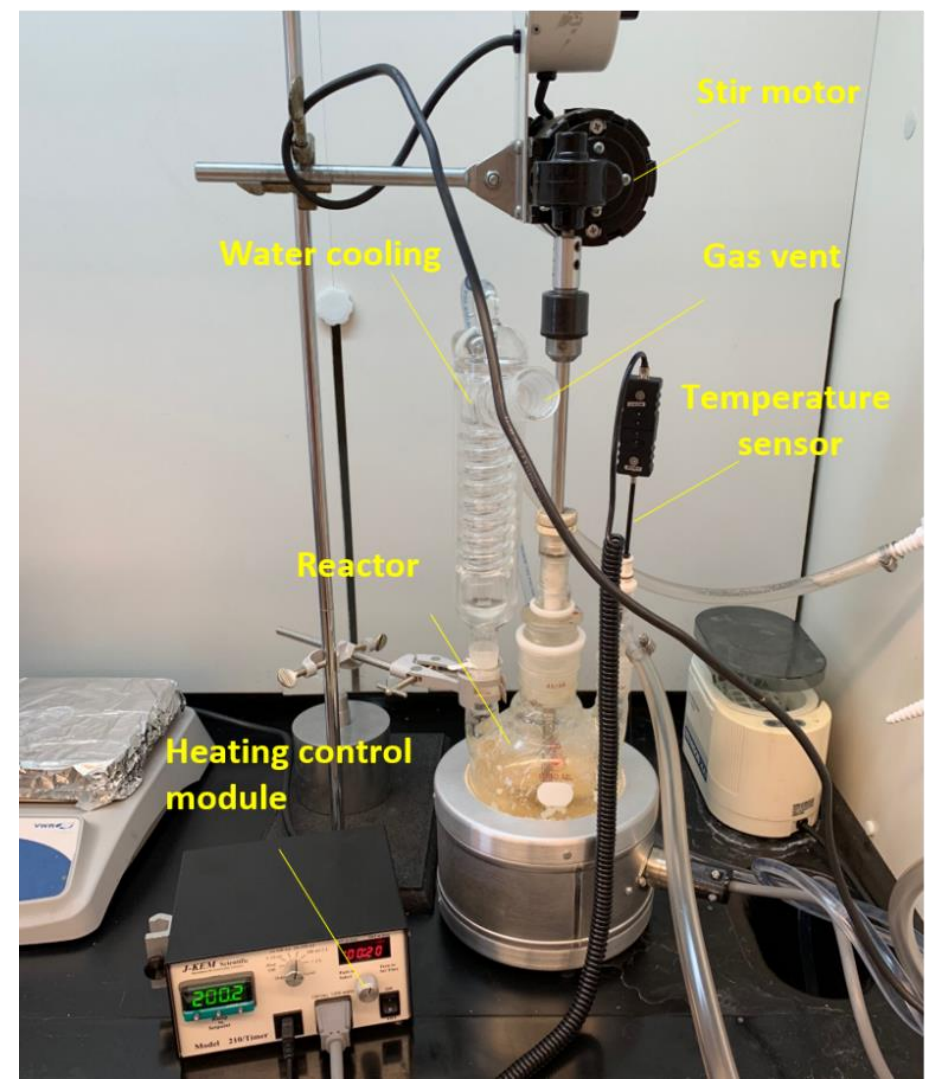

Figure 3. 27 Image of PLA-PEG copolymer synthesis setup

Table 3. 2 Copolymer synthesis component mass ratio

\begin{tabular}{ccc}
\hline Copolymers & PLA (g) & PEG (g) \\
\hline PLA-PEG (75:25) & 112.5 & 37.5 \\
\hline PLA-PEG (70:30) & 105 & 45 \\
\hline PLA-PEG (60:40) & 90 & 60 \\
\hline
\end{tabular}


Some non-polar organic solvents such as chlorobenzene $\left(B P=131^{\circ} \mathrm{C}\right), 1,2-$ dichloroethane $\left(\mathrm{BP}=84^{\circ} \mathrm{C}\right)$, acetone $\left(\mathrm{BP}=57^{\circ} \mathrm{C}\right)$, and chloroform $\left(\mathrm{BP}=61^{\circ} \mathrm{C}\right)$ were trialed to dissolve PLA and copolymers. All the solutions were vortex stirred for 3 minutes for pre-mix and followed by ultrasonication for 3 hours at room temperature. Those solvents that could dissolve the polymer and copolymers at a concentration larger than $20 \%$ were chosen to draw micro-fibers.

\subsubsection{Scaffold Fabrication}

\subsubsection{Fabrication of Single Suspended Fiber}

During the single suspended fiber fabrication process (Figure 3.28), the polymer solution was expelled to form a polymer droplet that makes contact with the substrate. The dispensing tip with polymer droplet then immediately was lifted $1 \mathrm{~mm}$ and laterally translated with a constant traveling velocity to the predefined termination location on the other side of the substrate. The polymer droplet thinned and elongated to form a filament by surface tension driven necking as defined by the capillary drying process. Finally, the polymer solution was expelled again, and the dispensing tip lowered down $1 \mathrm{~mm}$ to establish ending contact point. 


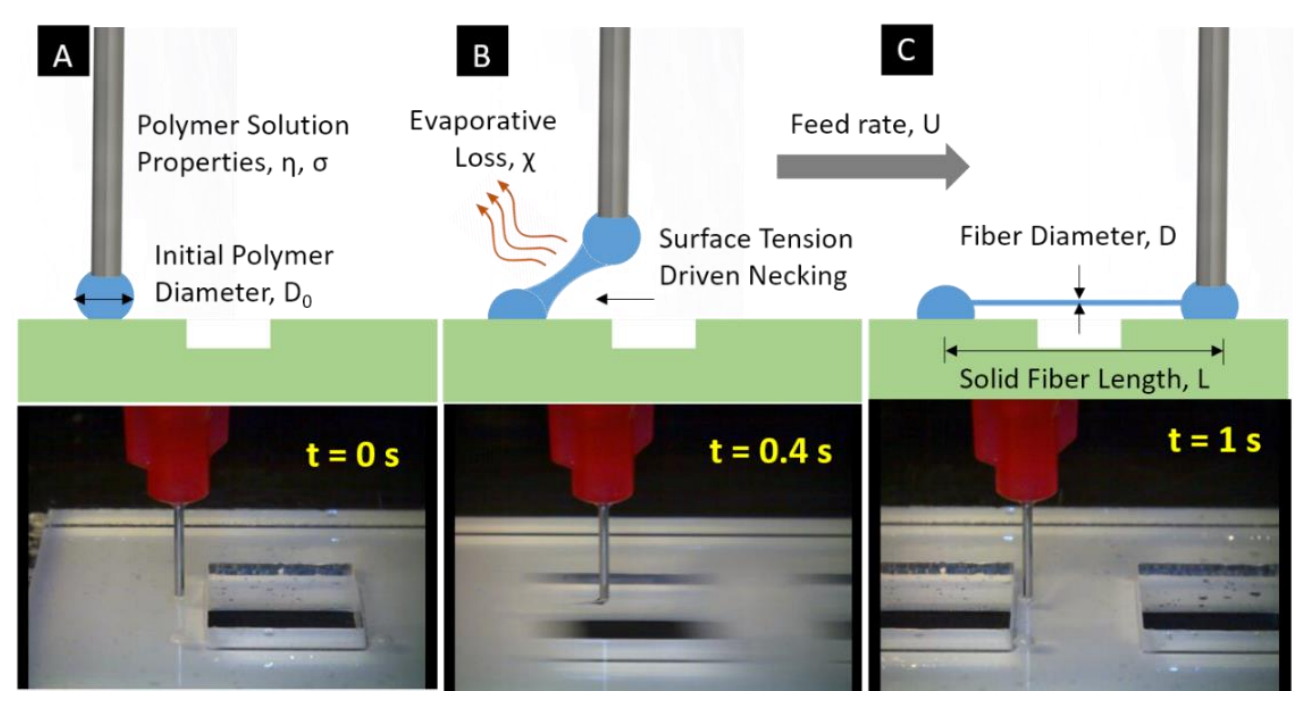

Figure 3. 28 Schematic illustrating (top) and real images (bottom) of the directwriting process. A) The polymer solution was expelled and contacted with the substrate. B) The dispensing tip was translated to a predefined point while the polymer filament undergoes surface tension driven necking. C) The polymer solution was expelled again to establish the ending contact point.

\subsubsection{Initial Polymer Drop Size and Needle-to-Substrate Gap}

The initial polymer drop size and the needle-to-substrate gap was determined experimentally by using USB-camera to capture and measure the resulting polymer drop images. Four different polymer solution drops were expelled under different valve open time: $0.02 \mathrm{~s}, 0.05 \mathrm{~s}, 0.1 \mathrm{~s}$, and $0.5 \mathrm{~s}$. The needle-tosubstrate gap was adjusted from 0 to $600 \mu \mathrm{m}$.

\subsubsection{Fabrication of Branched Structure}

The branch structure was fabricated as Figure 3.29. First, a single suspended fiber was fabricated across an acrylic frame (Figure 3.29A). Then, another fiber was drawn over top of the first fiber and lowered $2 \mathrm{~mm}$ to attach the 
two fibers (Figure 3.29B-C). Finally, the needle was lifted and translated to the ending point to finish the structure construction (Figure 3.29D). The attachment of the two fibers was in a very short period ( 1 second) so that both fibers still possess some solvent to promote the adhesion between the two fibers.
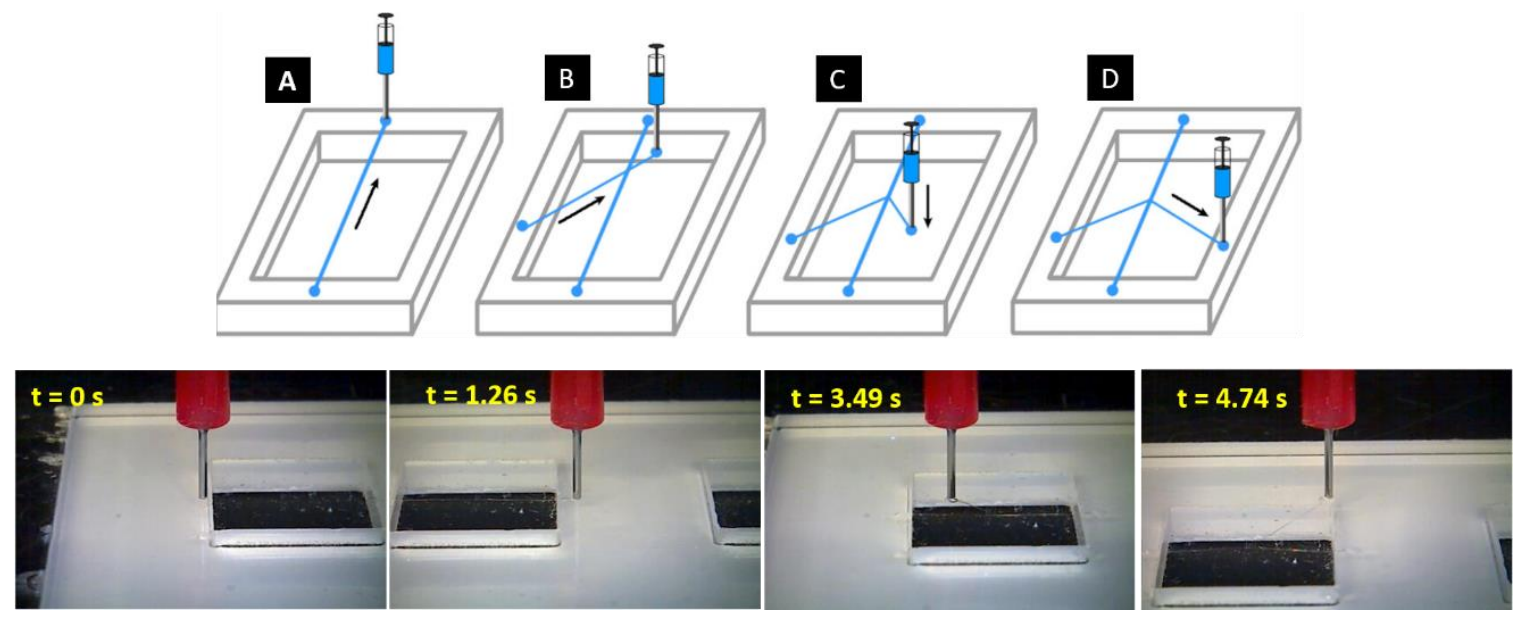

Figure 3. 29 Schematic illustrating and real images of the fabrication of the branched structure

\subsubsection{Empirical Model Generation}

\subsubsection{Theoretical Model}

A direct-written fiber is created by thinning and elongation from the bulk polymer solution, thus, understanding the polymer solution rheological properties is essential to the capillary thinning process. McKinley et al. developed an early model and showed that the filament would thin until capillary breakup when the filament forms[197]. The mathematical model is given below: 


$$
D_{(t)}=D_{1}-\frac{(2 X-1)}{3} \frac{\sigma}{\eta} t
$$

Where $D_{(t)}$ is the diameter of polymer filament, $D_{1}$ is the initial diameter of the polymer filament, $\mathrm{X}$ is a constant which determined to be 0.7127 and 1 by two different groups[198, 199], $\sigma$ is the surface tension, $\eta$ is the Newtonian viscosity, and $t$ is the filament formation time. This model illustrates the relationship between the final polymer fiber diameter, the physical properties of the polymer, and operation time. However, the high volatility for the solvent of the polymer solution has not been considered.

Tripathi et al. further proposed a theory that introduces the evaporation rate of the solvent into the model[200]. For a Newtonian solution, the equilibrium fiber diameter $D_{\infty}$ is given as below:

$$
\begin{gathered}
D_{\infty}=D_{1} e^{-0.035 / P} \\
P=\frac{\eta \chi}{\sigma}
\end{gathered}
$$

Where $P$ is defined as a dimensionless Processability parameter and $X$ is the evaporation rate, also known as polymer solution's mass transfer coefficient. The model clearly shows that the high viscosity and high volatility will resist the thinning process while the surface tension will induce the thinning process.

All the above models are established based on a simple polymer thinning process with constant length and zero velocity, but the direct-writing process has the ability to run at a different velocity and different length. The polymer fiber 
formation affected by an increased number of variables; thus, the Processability parameter model should be augmented with additional dimensionless parameters to solve this problem. According to the Buckingham Pi theorem, given a relation among $n$ parameters of the form:

$$
g\left(q_{1}, q_{2}, \cdots, q_{n}\right)=0
$$

The $n$ parameters may be grouped into $n-m$ independent dimensionless ratios, or $\Pi$ parameters, expressible in function form by:

$$
G\left(\Pi_{1}, \Pi_{2}, \cdots, \Pi_{n-m}\right)=0
$$

Which $m$ is usually equal to a minimum number of independent dimensions required to specify the dimensions of all the parameters.

Carefully inspecting the direct-writing process described in Figure. 3.28, one dependent variable (fiber diameter, $D_{\infty}$ ) and five independent variables are involved: the polymer solution viscosity $(\eta)$, polymer solution surface tension $(\sigma)$, mass transfer coefficient $(X)$, feed rate $(U)$, and fiber length $(L)$. MLt (mass, length, and time) was selected as fundamental dimensions set. Thus, this dimensional analysis could be grouped into three dimensionless parameters $(6-3=3)$.

Except for the Processability parameter, two additional dimensionless parameters were introduced to the model to define the fiber drawing process: Capillary number $\left(\mathrm{C}_{\mathrm{a}}\right)$ and Aspect ratio $(\Lambda)$, given as below:

$$
C_{a}=\frac{\eta U}{\sigma}
$$




$$
\Lambda=\frac{L}{D_{0}}
$$

Where $U$ is drawing velocity (feed rate) and $D_{0}$ is the initial diameter of the polymer droplet.

\subsubsection{Design of Experiment}

The design of experiments was conducted by using Minitab with two important aims. The first aim was to investigate the influences of the four factors (solution concentration, feed rate, the inner diameter of the needle (ID) and fiber length) on the yield of the fiber. The second aim was to analyze the effect of the $\mathrm{P}-\mathrm{C}_{\mathrm{a}}-\wedge$ dimensionless system on the diameter of the direct drawing fiber and further decide the optimal process parameters for to generate biopolymer fibers with diameters within microvascular range (5 - 20 $\mu \mathrm{m})$. The experiment design with levels of factors is shown in Table 3.3.

Table 3. 3 Selected factors and their levels used in the design of experiments

\section{Levels}

\section{Factors}

\begin{tabular}{ll}
\hline No. & Variables
\end{tabular}

\begin{tabular}{lcl}
\hline A, Concentration (wt.\%) & 3 & $15,16,17$ \\
B, Feed Rate $(\mathrm{mm} / \mathrm{s})$ & 3 & $100,200,300$ \\
C, Needle ID (mm) & 4 & $0.10,0.15,0.20,0.25$ \\
D, Fiber length (mm) & 12 & $2,4,6,8,10,12,14,16,18,20,30,40$ \\
\hline
\end{tabular}




\subsubsection{Model Generation}

The physical properties of the gelatin solution, such as viscosity, surface tension, and evaporation rate, were measured by the previously described methods in section 3.2. Three dimensionless parameters--Processability parameter $(P)$, Capillary number $\left(C_{a}\right)$, and Aspect ratio $(\Lambda)$--were calculated from the determined polymer solution physical properties and the operational parameters. The finished fibers array was sputter coated with a thin layer $(t=10$ $\mathrm{nm}$ ) of gold-palladium alloy and imaging by SEM. The diameters of each fiber were measured and recorded at the middle point as well as $200 \mu \mathrm{m}$ from the initiating and terminating side; the average of these values was used as the final fiber diameter. An ANOVA based on the gelatin characterization data was performed using Minitab software to find out the factors that significantly affect the response. Only those significant factors determined by the ANOVA were chosen to perform the linear regression analysis to generate the empirical model and contour plots. The empirical model included three dimensionless parameters $\left(P-C_{a}-\Lambda\right)$, which related micro-fiber diameter to five independent variables (viscosity, surface tension, solvent evaporation rate, fiber length, and feed rate). Furthermore, the adequacy of the regression model also was checked through Minitab.

\subsubsection{Empirical Model Validation}

Because the empirical model was generated using only one biopolymer (gelatin), it is necessary to perform experiments with other biopolymers, 
composites, and copolymers in order to validate the empirical model. A list of the biopolymers, solvents and their concentrations is shown in Table 3.4.

Table 3. 4 Biopolymers used to validate the gelatin-based empirical model

\begin{tabular}{|c|c|c|}
\hline Biopolymer composition & Solvent & $\begin{array}{c}\text { Concentration } \\
\text { (wt.\%) }\end{array}$ \\
\hline PLGA & TFE & $25 \%$ \\
\hline PLGA & TFE & $26 \%$ \\
\hline Gelatin/PLGA (70:30) & TFE & $17 \%$ \\
\hline Gelatin/PLGA (70:30) & TFE & $18 \%$ \\
\hline Gelatin/PLGA (50:50) & TFE & $18 \%$ \\
\hline Gelatin/PLGA (50:50) & TFE & $19 \%$ \\
\hline PLA & Chloroform & $28 \%$ \\
\hline PLA & Chloroform & $29 \%$ \\
\hline PLA-PEG (75:25) & Chloroform & $20 \%$ \\
\hline PLA-PEG (75:25) & Chloroform & $21 \%$ \\
\hline PLA-PEG (70:30) & Chloroform & $30 \%$ \\
\hline PLA-PEG (70:30) & Chloroform & $31 \%$ \\
\hline
\end{tabular}


Based on the previous optimal results, the needle with an inner diameter of $0.25 \mathrm{~mm}$ was chosen for the fiber fabrication. Micro-fibers array with lengths of 4 , 6 , and $8 \mathrm{~mm}$ (each length for three fibers) were drawn at a feed rate of $200 \mathrm{~mm} / \mathrm{s}$ for all biopolymer solutions. The biopolymer solution physical parameters and dimensionless parameters $\left(P-C_{a}-\Lambda\right)$ were measured and calculated as previously described for the gelatin solution. The prediction data was then gathered by input of the dimensionless parameters into the empirical model. The experimental fiber diameters were also measured in three points by SEM as previously introduced in section 3.3.3.3. Then the experimental data was compared with the values predicted by the empirical model.

\subsubsection{Cells Seeding on Scaffold}

\subsubsection{Cell Culture and Reagents}

Human Dermal Microvascular Endothelial Cells (HDMECs) (PromoCell $\mathrm{GmbH}$, Heidelberg, Germany) were cultured in Endothelial Cell Growth Medium MV kit with growth supplement containing Fetal Bovine Serum $(0.05 \mathrm{~mL} / \mathrm{mL})$, Epidermal Growth Factor ( $5 \mathrm{ng} / \mathrm{mL}$ ), Basic Fibroblast Growth Factor (10 $\mathrm{ng} / \mathrm{mL})$, Vascular Endothelial Growth Factor $165(0.5 \mathrm{ng} / \mathrm{mL})$, Ascorbic Acid $(1 \mu \mathrm{g} / \mathrm{mL})$, and Hydrocortisone $(0.2 \mu \mathrm{g} / \mathrm{mL})$ (PromoCell $\mathrm{GmbH}$, Heidelberg, Germany). The antibiotic-antimycotic containing 10,000 units $/ \mathrm{mL}$ penicillin and $10,000 \mu \mathrm{g} / \mathrm{mL}$ streptomycin (GE Healthcare Bio-Sciences, Pittsburgh, PA) was also added to the medium kit with the growth supplement. 0.05\% Trypsin/0.53 mM EDTA (Mediatech, Inc. Manassas, VA) was used to detach the cells when subculturing the cells. 
One vial of cryopreserved HDMECs ( 500,000 cells) was thawed for 3 minutes in a water bath $\left(37^{\circ} \mathrm{C}\right)$. Then, the cell solution was pipetted into a T-25 flask with $8 \mathrm{~mL}$ of growth medium and cultured for 2 days in the incubator $\left(37^{\circ} \mathrm{C}\right.$, $5 \% \mathrm{CO}_{2}$ ). Once it had reached $90 \%$ confluency, the cells were passaged into a T75 flask by using $1 \mathrm{~mL}$ of $0.05 \%$ trypsin solution. The media was changed every two days. In $90 \%$ confluency, the cells were passaged again by $2 \mathrm{~mL}$ of $0.05 \%$ trypsin solution and $2 \mathrm{~mL}$ of media was added to obtain a total of $4 \mathrm{~mL}$ cells suspension solution. Cellometer was utilized at least twice to check the cell density, then the cells with known density could be seeded on the substrates of interest. All cells in this study were used between passages 3 and 8 .

\subsubsection{Biodegradation Test}

Generally, it will take 6 months to 2 years to fully degrade PLA and PLGA[56]. Thus, it is very important to understand the degradation behavior of the biopolymers, composites, and copolymers. Using a bench top compression molding (Figure 3.30A), each biopolymer, composites, and copolymer were cast into several $15 \mathrm{~mm}$ diameter by $10 \mathrm{~mm}$ high cylinders (Figure 3.30B). Each sample cylinder was placed in a Petri dish and incubated with $15 \mathrm{~mL}$ of $1 \mathrm{X}$ phosphate-buffered saline (PBS) solution at body temperature $\left(37^{\circ} \mathrm{C}\right)$ for up to 8 weeks. PBS solution was changed every week to eliminate the acidic biodegradation products. At the end of each week, the sample cylinders were rinsed with DI water and vacuum dried for 30 minutes, then the mass lost were recorded to obtain the short-term degradation profiles. 


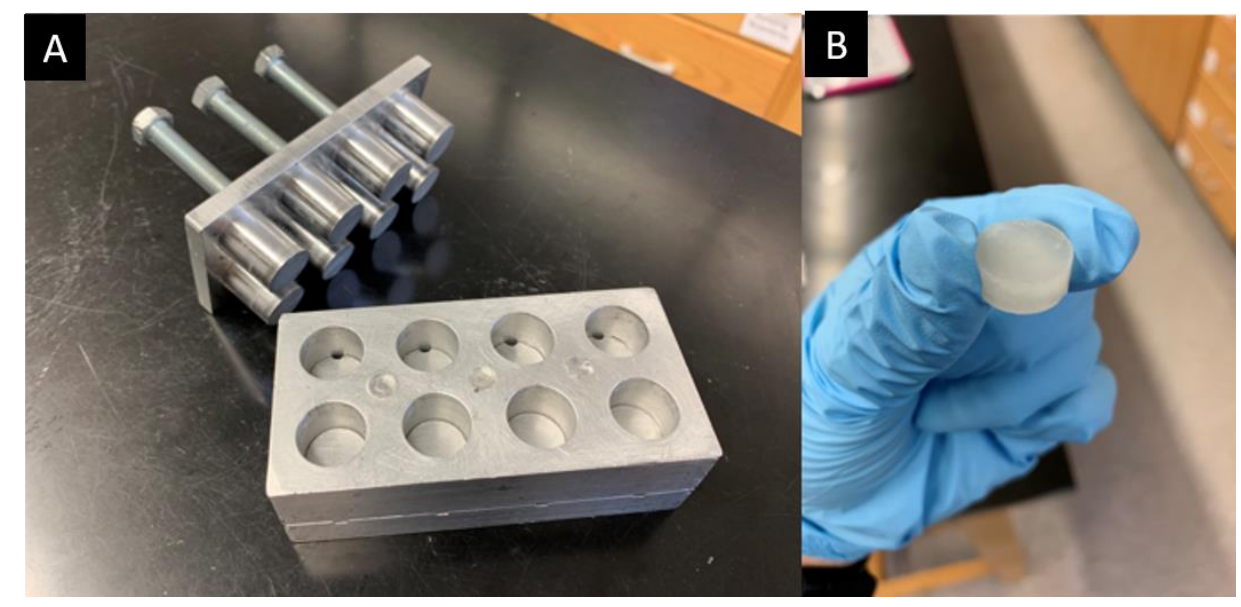

Figure 3. 30 Image of sample molding: A) Benchtop compression molding, B) example of molded PLA-PEG (75:25) sample cylinder

\subsubsection{Biopolymers Cellular Adhesion/Viability Test}

To evaluate if the chosen biopolymers, composites, and copolymers were suited for the HDMECs growth, a cellular adhesion test was performed. $400 \mu \mathrm{L}$ of $6 \%$ biopolymers solutions were deposited in the 24 -well plate to form films. The films were then heated to $65^{\circ} \mathrm{C}$ for 30 minutes and then left for 3 days in vacuum at room temperature to further remove residual solvent. Each film was sterilized by UV light exposure for 1 hour, coated with $200 \mu \mathrm{L}$ of $5 \mu \mathrm{g} / \mathrm{mL}$ fibronectin aqueous solution, and incubated for 1 hour at $37^{\circ} \mathrm{C}$. After aspirated the fibronectin solution and washed with 1X PBS twice, HDMECs were seeded on each of the films at a density of 40,000 cells $/ \mathrm{cm}^{2}$ (76,000 cells for each plate of a 24 -well plate, bottom surface area $=1.9 \mathrm{~cm}^{2}$ ) and incubated for 24 hours at $37^{\circ} \mathrm{C}$. To count the quantity of the adhered cells on the film, each sample was fixed and stained with 4,6diamidino-2-phenylindole (DAPI). To check the cell viability, each sample was stained with a live/dead assay. The details of the staining procedure will be 
introduced on section 3.3.5.6. Fluorescent images were taken by the fluorescent microscope and analyzed by ImageJ software.

\subsubsection{Cell Seeding Conditions}

The basic idea of seeding cells on the scaffold is to submerge the scaffold in cell suspension and growth medium within a container, such as a 24-well plate or a chambered cover glass system. Three cell seeding conditions were studied to develop the optimal protocol for cell seeding onto the scaffold: (1) cells blocking, (2) adhesion promoters, and (3) cell seeding density.

To reduce the attached cells on the bottom of the container, bovine serum albumin (BSA) was used to block the bottom of the container. $0.2 \mathrm{~mL}$ of $0.01 \%$, $0.1 \%$, and $1 \%$ of BSA (Sigma, St. Louis, MO) solution in 1X PBS was first added into the 24-well plate to cover the bottom of the well and incubated for 1 hour at $37^{\circ} \mathrm{C}$. After aspirating the BSA solution, HDMECs were then seeded at a density of 40,000 cells $/ \mathrm{cm}^{2}$ and incubated for 24 hours at $37^{\circ} \mathrm{C}$.

Gelatin attachment factor and fibronectin are often used to coat culture surfaces to enhance the adhesion and growth of microvascular endothelial cells[201]. To evaluate the effectiveness of the cell adhesion promoters, cellular adhesion quantification experiments were conducted on both polymer films and fibers. Polymer films were fabricated in the 24-well plate through solvent casting from $400 \mu \mathrm{L}$ of $6 \%$ PLA-PEG (75:25) copolymer solution in chloroform. Polymer fibers were fabricated on a custom cell culture fiber frame with a recessed platform via the direct-write system from 20\% PLA-PEG (75:25) copolymer solution in chloroform. Each film and fiber was coated with $200 \mu \mathrm{L}$ of $1 \times$ gelatin attachment 
factor (AF, Cascade, Thermo Fisher Scientific, Waltham, MA), $5 \mu \mathrm{g} / \mathrm{mL}$ fibronectin (FN, Thermo Fisher Scientific, Waltham, MA) aqueous solution, and combined two promoters and then incubated for 1 hour at $37^{\circ} \mathrm{C}$. After aspirating the adhesion promoter solution, HDMECs were then seeded at a density of 40,000 cells $/ \mathrm{cm}^{2}$ and incubated for 24 hours at $37^{\circ} \mathrm{C}$.

The micro-fiber scaffold will ultimately be submerged in the cell suspension during experimentation to capture the floating cells randomly. Thus, the seeding density may affect the final output of the cell adhesion. Three parallel PLA-PEG (75:25) micro-fibers with a distance of $2 \mathrm{~mm}$ were fabricated on the custom cell culture fiber frame with a recessed platform via the direct-write system. Each fiber was coated with $200 \mu \mathrm{L}$ of $5 \mu \mathrm{g} / \mathrm{mL}$ fibronectin aqueous solution and incubated for 1 hour at $37^{\circ} \mathrm{C}$. Then, the fibronectin-coated fibers were transferred into a $0.1 \%$ BSA (incubated for 1 hour at $37^{\circ} \mathrm{C}$ ) pre-treated 24-wall plate. HDMECs were then seeded at a series seeding density: 40,000 cells $/ \mathrm{cm}^{2}, 60,000$ cells $/ \mathrm{cm}^{2} .80,000$ cells $/ \mathrm{cm}^{2}$, and 100,000 cells $/ \mathrm{cm}^{2}$. The cells were all incubated for 24 hours at $37^{\circ} \mathrm{C}$.

For each of the experiments described, the number of attached cells was compared by the fluorescent images after the cells were fixed and stained with DAPI. Stained nuclei were counted by ImageJ software. Student t-tests were performed to identify the optimal seeding recipe.

\subsubsection{Cell Seeding on the Scaffold}

The fabricated scaffold on the custom frame was left under vacuum for overnight to make sure no residual solvent was left. Firstly, the custom frame was flipped (the recessed platform toward down) and put into a 24-well plate followed 
by exposed under UV light in the laminar-flow hood for 1 hour. After the sterilization, the scaffold was covered with $0.1 \mathrm{~mL}$ of $5 \mu \mathrm{L} / \mathrm{mL}$ fibronectin solution and incubated at $37^{\circ} \mathrm{C}$ for 1 hour. At the same time, another 24 -well plate was covered with 0.1 $\mathrm{mL}$ of $0.1 \% \mathrm{BSA}$ solution and put into the incubator for 1 hour. Then, the fibronectin solution and BSA solution were aspirated along the inner wall of the well, and the fibronectin-coated scaffold was transferred carefully by a sterile tweezer to the BSA-coated well. This process needs to be completed extremely carefully because the scaffold is very easy to be broken by the vacuum or the surface tension induced by the meniscus of residual liquid. The procedure of 3.3.5.1 was followed to obtain enough HDMECs suspension with known cell density. For a 24-well plate (bottom surface area $=1.9 \mathrm{~cm}^{2}$ ), each wall needed to be seeded with $1.524 \times 10^{5}$ cells to reach a cell density of 80,000 cells $/ \mathrm{cm}^{2}$. The desired cell suspension volume was calculated based on the cell density. Then, the cell suspension was gently seeded on the scaffold, and the fresh media was added to the working volume $(0.5 \mathrm{ml}$ for 24 wall plate). The 24 -well plate was placed into the incubator while slowly rocking on a nutating mixer (Thermo Fisher Scientific, Figure 3.31) for 1 day to improve the cell adhesion, followed by 1 day of incubation without the rocking. 


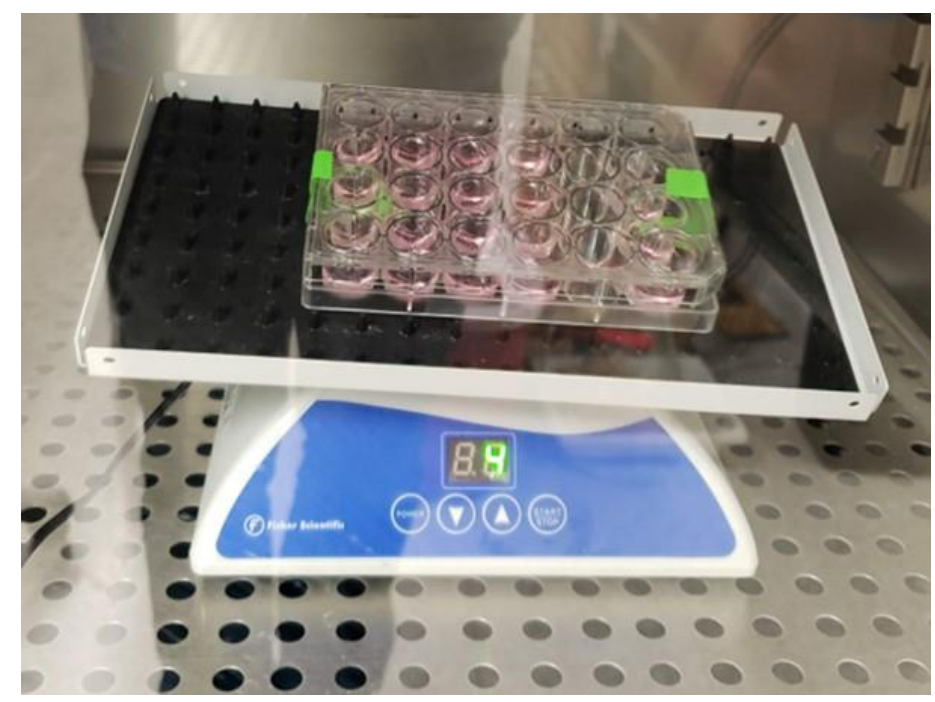

Figure 3. 31 Cells seeded on the scaffold with slow rocking on a nutating mixer in the incubator

\subsubsection{Immunofluorescent Staining}

Unless otherwise noted, all the samples observed under the fluorescent microscope and confocal microscope were first washed by 1 X PBS for 1 minute, fixed by $3.7 \%$ formaldehyde for 10 minutes at room temperature, and then permeabilized in $0.1 \%$ Tween 20 for 5 minutes.

\subsection{DAPI and Rhodamine Phalloidin Staining}

The nucleus was stained by DAPI ( $300 \mu \mathrm{M}$, courtesy of Dr. Patricia Soucy) and the cytoskeleton was stained by rhodamine phalloidin (200 units/ml, Invitrogen, Thermo Fisher Scientific, Waltham, MA). The DAPI and rhodamine phalloidin was thawed, then diluted in 1 X PBS (1:5) and 0.1\% BSA solution (1:50), respectively. Each sample was incubated at $37^{\circ} \mathrm{C}$ for 30 minutes in $0.1 \mathrm{~mL}$ of the diluted rhodamine phalloidin solution. The sample was covered properly to avoid solution 
evaporation. After removing the rhodamine phalloidin solution and washing the sample twice with $1 \mathrm{X}$ PBS, $0.1 \mathrm{~mL}$ of diluted DAPI solution was added to cover the sample and standing for 3 minutes at room temperature. After washing three times, $0.5 \mathrm{~mL}$ fresh 1X PBS was added to submerge the sample.I Imaging on the fluorescent or confocal microscope occurred immediately after. All the steps above were finished in the dark to protect the fluorescent dyes from light exposure. The best staining result was achieved by performing the fixation and staining on the same day.

\subsection{VE-Cadherin Staining}

VE-Cadherin staining was used to analyze the cell-cell junctions of cells adhered to the scaffold. Firstly, the primary antibody was prepared by diluting rabbit anti-VE cadherin (AB-33168, courtesy of Dr. Patricia Soucy) in 0.1\% BSA (1:50). The secondary antibody was prepared by adding goat-anti-rabbit IgG Alexa Fluor 488 (A-11034, courtesy of Dr. Patricia Soucy) to $0.1 \%$ BSA with a dilution of 1:40. Then, each sample was incubated at $37^{\circ} \mathrm{C}$ for 30 minutes in $0.1 \mathrm{~mL}$ of the primary antibody. The sample was covered properly to avoid solution evaporation. Following 3 minutes of washing with $1 \mathrm{X}$ PBS, $0.1 \mathrm{~mL}$ of the secondary antibody was added to cover the sample and continued to incubate at $37^{\circ} \mathrm{C}$ for 30 minutes. After washing another 3 minutes with $1 \mathrm{X}$ PBS, the samples were visualized using the confocal microscope with $0.5 \mathrm{~mL}$ fresh PBS as an anti-fade solution. All the steps above were finished in the dark to protect the antibodies from the light exposure. 


\subsection{Live/Dead Assay}

Live/dead assay (Invitrogen MP 03224, courtesy of Dr. Patricia Soucy) was used to study the cellular adhesion on different biopolymers as well as to check the viability of the cells on the scaffold in the ECM. The live cells are distinguished by the presence of ubiquitous intracellular esterase activity, determined by the enzymatic conversion the virtually nonfluorescent cell-permanent Calcein AM to the fluorescent calcein. EthD-1 enters cells with damaged membranes and undergoes a 40 -fold enhancement of fluorescence upon binding to nucleic acids. EthD-1 is excluded by the intact plasma membrane of live cells.

The sample for this experiment cannot be fixed. In addition, the staining solution should be made fresh each time in the laminar-flow hood and need to be protected from light exposure. By calculating the desired volume of staining solution, ethidium homodimer-1 (2 mM) was diluted to $4 \mu \mathrm{M}$, and Calcein $\mathrm{AM}$ (4 $\mathrm{mM}$ ) was diluted $2 \mu \mathrm{M}$ in the same vial in serum-free media 200. After gently washing the cells with serum-free media 200 twice, $0.1 \mathrm{~mL}$ of the staining solution was added to cover the whole sample and incubated at $37^{\circ} \mathrm{C}$ for 30 minutes. Then, the samples were ready to be viewed under the fluorescent microscope.

\subsubsection{Sample Preparation for SEM}

All the chemicals used in this experiment were purchased from Electron Microscopy Sciences (Hatfield, PA). The experiment needed to be conducted in a fume hood for safety purposes. The cell-covered scaffold was gently washed with 1 X PBS for 1 minute, then fixed in $2 \%$ paraformaldehyde, $2 \%$ glutaraldehyde, 
$0.1 \mathrm{M}$ sodium cacodylate, and $3 \mathrm{mM}$ sodium chloride $(\mathrm{pH} 7.4)$ at room temperature for 1 hour while slowly rocking. After washing with $1 \mathrm{X}$ PBS for 10 minutes, the sample was post-fixed in $0.8 \%$ potassium ferrocyanide-reduced $1 \%$ osmium tetroxide, $0.1 \mathrm{M}$ sodium cacodylate, and $3 \mathrm{mM}$ sodium chloride for 1 hour on ice in the dark. Following the DI water rinse (twice, 5 minutes each), the sample was then placed in $2 \%$ uranyl acetate-aqueous $(0.22 \mu \mathrm{m}$ filtered) for 1 hour at room temperature in the dark. Then, the sample was dehydrated through a graded series of ethanol with the following schedule: washed by $50 \%, 70 \%$, and $90 \%$ ethanol 5 minutes each, then washed by $100 \%$ ethanol three times for 5 minutes each. Upon completing the ethanol treatment, the sample was further dehydrated by washing with $0.5 \mathrm{~mL}$ hexamethyldisilazane (HMDS) for 5 minutes. After the HMDS was drained, the sample was placed in a desiccator overnight until it was completely dried. The sample received a $10 \mathrm{~nm}$ thick gold-palladium sputter coating before being observed under the SEM.

\subsubsection{Cells and Scaffold Embedded in ECM}

\subsubsection{Collagen Gel Solution Preparation}

Type I collagen (rat tail, $8.34 \mathrm{mg} / \mathrm{mL}$, Corning, NY) was used to work as ECM in this project. To prepare a $1 \mathrm{mg} / \mathrm{mL}$ collagen gel solution, the following components (courtesy of Dr. Patricia Soucy, Table 3.5) were added into a tube on ice and mixed for 1 minute with a pipette. $\mathrm{NaOH}$ was used to adjust the $\mathrm{pH}$ to 7.4 and DI water was used to make the desired volume. The collagen gel solution was made freshly 5 minutes before encapsulating the cell-covered scaffold. 
Table 3. 5 Collagen Gel Solution Components

\begin{tabular}{ccc}
\hline Components & $\begin{array}{c}\text { Stock } \\
\text { Concentration }\end{array}$ & $\begin{array}{c}\text { Example volume for } \\
\mathbf{4 0 0} \mu \mathrm{L} \text { solution }\end{array}$ \\
\hline $\mathrm{NaHCO}_{3}$ & $10 \mathrm{mg} / \mathrm{ml}$ & $20 \mu \mathrm{L}$ \\
Collagen & $8.34 \mathrm{mg} / \mathrm{ml}$ & $48 \mu \mathrm{L}$ \\
PBS & $10 \mathrm{X}$ & $40 \mu \mathrm{L}$ \\
HEPES & $2 \mathrm{M}$ & $2 \mu \mathrm{L}$ \\
$\mathrm{NaOH}$ & $1 \mathrm{M}$ & $15 \mu \mathrm{L}$ \\
$\mathrm{DI}$ water & - & $275 \mu \mathrm{L}$ \\
\hline
\end{tabular}

\subsubsection{Gelation and Cell Culture}

To prevent the cells on the bottom of the well plate from migrating to the deposited collagen gel and interfering with imaging, the cell-covered scaffold needed to be transferred into a new container for ECM gelation. First, gently aspirated the medium along the inner wall of the 24-well plate to avoid damage to the scaffold induced by the liquid surface tension. Then, the cell-covered scaffold was washed by 1 X PBS solution and carefully transferred into a new 24-well plate or chambered cover glass system by a sterile tweezer. Slowly, $0.1 \mathrm{~mL}$ freshly prepared collagen solution was added to fully cover the scaffold; samples were incubated for 35 minutes at $37^{\circ} \mathrm{C}$. After gelation finished, the desired amount of 
media was added to a 24 -well plate $(0.5 \mathrm{~mL})$ or a chambered cover glass system $(1.5 \mathrm{~mL})$, and then continued to incubate for an additional 3 days. The media was changed every two days.

\subsubsection{Imaging Samples Preparation}

After the cells on the scaffold are cultured in the collagen gel for 3 days, fluorescent images were obtained by the fluorescent microscope and the confocal microscope to observe the cells growth in the ECM. The staining procedures were similar to the previously described in section 3.3.5.6. However, the fixation time, washing time, and staining time need to be doubled due to the slow diffusion in the collagen gel.

To observe the ECM-cells-scaffold interaction and evaluate the scaffold degradation, SEM cross-sectional images of the scaffold embedded in collagen gel were captured. The procedure of section 3.3.5.7 was followed, butafter the HMDS treatment, the collagen gel film was still soft. If the gel film was left on the frame until it was completely dry, the film may tear or warp and would not be usable. To safely preserve the sample, the four sides of the gel film were gently cut and carefully transferred on to a PTFE surface. After the gel film completely dried, it was cut perpendicular to the scaffold axis with a sharp blade. The sample was attached onto a vertical SEM specimen stage and a $10 \mathrm{~nm}$ thick gold-palladium sputter coating was applied before being observed under the SEM. 


\section{CHAPTER 4: RESULTS AND DISCUSSION}

In this chapter, the results associated with the specific aims outlined in the introduction will be examined. First, the fabrication results of the biopolymer microfibers and branch structures will be presented. Next, the direct-write process will be characterized through the design of experiments (DOE), and an analysis of variance (ANOVA) will be conducted to determine the significant factors that are affecting the micro-fiber diameter. In the course of this investigation, an empirical model will be developed and validated to predict the micro-fibers diameter through the dimensional analysis. Then, the growth of the Human Dermal Microvascular Endothelial Cells (HDMECs) on the scaffold will be evaluated after culturing for 2 days. Finally, the scaffold degradation and the ECM-cell-scaffold interactions will be evaluated once the cell-covered scaffold is embedded into the collagen gel.

\subsection{Fabrication of Biopolymer Micro-fibers and Branch Structures via 3-Axis} Robotic Dispensing System

It has been more than a decade since our group began to study the directwriting technique. Dr. Scott Berry first employed the Ultra-High Precision Micromilling Machine (UHPMMM) to generate micron-sized fibers from poly(methyl methacrylate) (PMMA) as well as a variety of biopolymers such as polycaprolactone (PCL), polylactic acid (PLA), and poly(lactic-co-glycolic acid) (PLGA)[181]. Furthermore, Dr. Hanwen Yuan utilized the 3-Axis robotic dispensing system to produce micro-/nano-fibers from PMMA and demonstrated the ability to 
reach a higher yield compared to using the previous UHPMMM method[202]. In this project, we adopted the 3-Axis robotic dispensing system and successfully fabricated precisely-positioned, suspended micro-fibers and branched structures with microvascular-scale diameters from several natural, synthetic, and composites biopolymers.

\subsubsection{Gelatin, PLGA, and Composites}

Before the micro-fibers were produced, the optimal solvent was firstly identified for gelatin since there are a limited number of polar organic solvents with moderate volatility. Table 4.1 shows the solvent and concentration trials for gelatin. The acetic acid solution was excluded due to the poor fiber drawing ability. 2,2,2trifluoroethanol (TFE) was ultimately chosen based on the increased solubility characteristics of the gelatin and the fiber drawing ability. If the concentration is too high, the solution was observed to be very thick. The majority of the drawing attempts at the higher concentrations showed tensile failure and began to coil due to the lack of solvent during the drawing (Figure 4.1A). On the contrary, if the concentration is too low, the solution was observed to be very thin. Most of the drawing attempts at the low concentration experienced breakup failure, which may be caused by not having enough polymer in the solution to form the micro-fiber (Figure 4.1B). 
Table 4. 1 Solvent and concentration trials for gelatin

\begin{tabular}{|c|c|c|c|}
\hline Concentration & Solvent & Solution comments & Fiber drawing ability \\
\hline $22 \%$ & \multirow{7}{*}{ TFE } & Two phases, form gel & $\bar{x}$ \\
\hline $20 \%$ & & Very thick gel & $x$ \\
\hline $18 \%$ & & Dissolve well, very thick & $x$ \\
\hline $17 \%$ & & Dissolve well, flow well & $\checkmark$ \\
\hline $16 \%$ & & Dissolve well, flow well & $\checkmark$ \\
\hline $15 \%$ & & Dissolve well, flow well & $\checkmark$ \\
\hline $14 \%$ & & Dissolve well, very thin & $\mathrm{X}$ \\
\hline $32 \%$ & \multirow{6}{*}{ AA } & Cannot dissolve, sediment & $\bar{X}$ \\
\hline $30 \%$ & & Very thick gel & $x$ \\
\hline $28 \%$ & & Dissolve well, very thick & $x$ \\
\hline $27 \%$ & & Dissolve well, flow well & $x$ \\
\hline $26 \%$ & & Dissolve well, flow well & $\checkmark$ \\
\hline $25 \%$ & & Dissolve well, very thin & $x$ \\
\hline
\end{tabular}




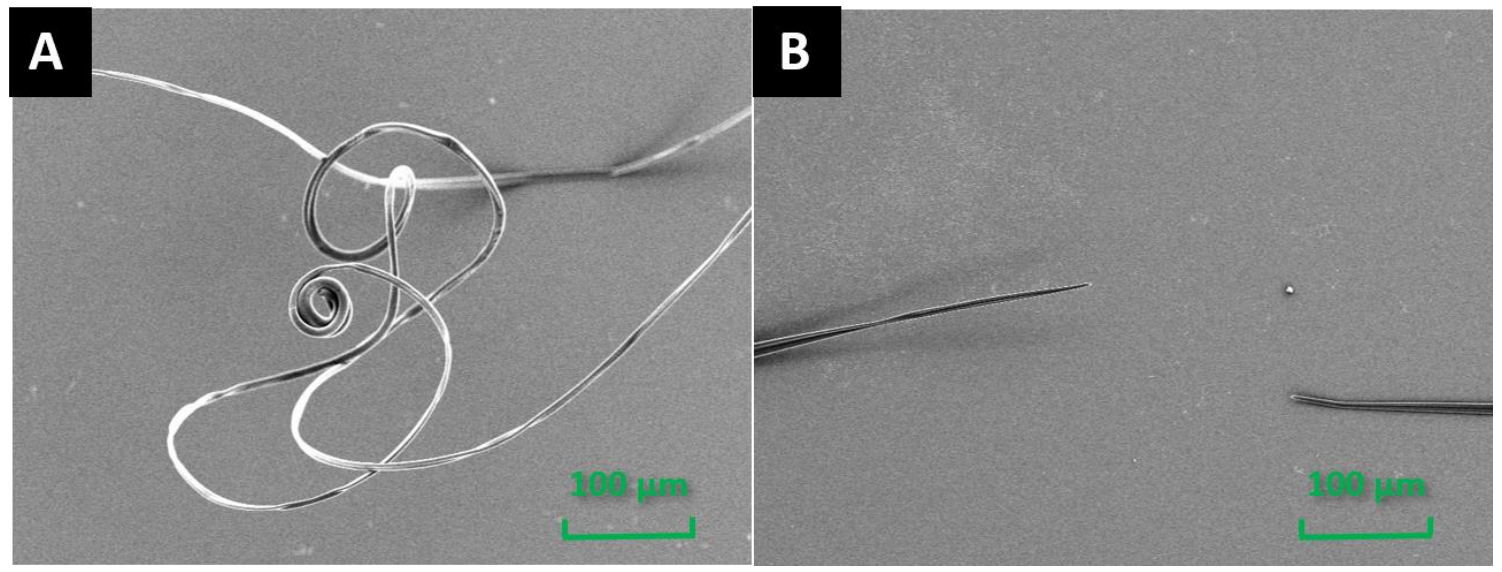

Figure 4. 1 SEM images of gelatin fibers drawn from the solutions which were: (A) very thick and $(B)$ very thin.

Once the solvent for gelatin was selected, the TFE solution concentrations for PLGA and gelatin/PLGA composites were also identified by trials, similar to the initial gelatin micro-fibers. These results are listed in Table 4.2.

Table 4. 2 TFE solution concentration trials for PLGA and gelatin/PLGA composites

\begin{tabular}{cccc}
\hline Concentration & concentration & Solution & Fiber drawing ability \\
\hline PLGA & $24 \%$ & Very thin & X \\
PLGA & $25 \%$ & Dissolve well & $\checkmark$ \\
PLGA & $26 \%$ & Dissolve well & $\checkmark$ \\
PLGA & $27 \%$ & Dissolve well & X \\
Gelatin/PLGA (70:30) & $17 \%$ & Dissolve well & $\checkmark$ \\
Gelatin/PLGA (70:30) & $18 \%$ & Dissolve well & $\checkmark$ \\
Gelatin/PLGA (70:30) & $19 \%$ & Dissolve well & X \\
\hline
\end{tabular}




\begin{tabular}{llll}
\hline Gelatin/PLGA (50:50) & $18 \%$ & Dissolve well & $\checkmark$ \\
Gelatin/PLGA (50:50) & $19 \%$ & Dissolve well & $\checkmark$ \\
Gelatin/PLGA (50:50) & $20 \%$ & Two phases & X \\
\hline
\end{tabular}

Suspended gelatin, PLGA, and their composite micro-fibers with microvascular-scale diameters were successfully fabricated by the direct-write technique via the 3 -axis robotic dispensing system. Figure 4.2A showed the gelatin droplet as the contact point and the end of the fiber, where the surface tension thinning began. It can also be observed in the PLGA fibers and gelatin/PLGA composite fibers. Figure 4.2B shows a single gelatin micro-fiber with a diameter of $14.46 \mu \mathrm{m}$ (processing factors: solution concentration $=17 \%$, needle $I D=0.25 \mathrm{~mm}$, feed rate $=200 \mathrm{~mm} / \mathrm{s}$, and fiber length $=6 \mathrm{~mm}$ ). Figure $4.2 \mathrm{C}$ shows one PLGA micro-fiber with a diameter of $6.88 \mu \mathrm{m}$ (processing factors: solution concentration $=27 \%$, needle ID $=0.20 \mathrm{~mm}$, feed rate $=300 \mathrm{~mm} / \mathrm{s}$, and fiber length $=8 \mathrm{~mm}$ ). Figure 4.2D shows a single gelatin/PLGA composites fiber with a diameter $7.15 \mu \mathrm{m}$ (processing factors: solution concentration $=$ total $16 \%$ with gelatin/PLGA ratio of $70: 30$, needle ID $=0.25 \mathrm{~mm}$, feed rate $=100 \mathrm{~mm} / \mathrm{s}$, and fiber length $=6 \mathrm{~mm}$ ). According to the SEM images, these different biopolymer microfibers all show a smooth cylindrical and bead-free morphology. The addition of PLGA to gelatin does not affect the surface microstructure. 

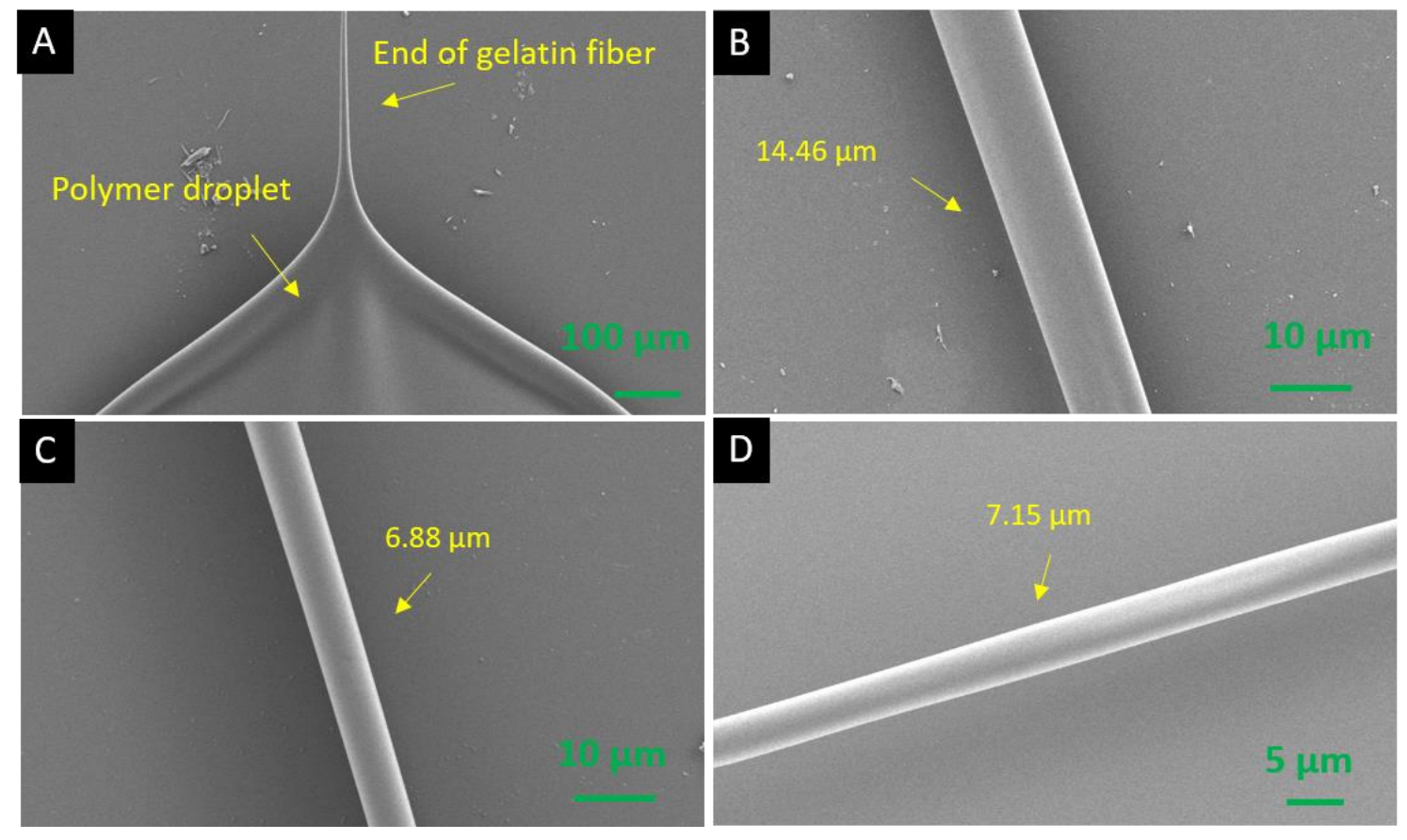

D

Figure 4. 2 SEM images of the biopolymer micro-fibers fabricated with the 3-Axis robotic dispensing robotic system. A) End of gelatin fiber, B) gelatin micro-fiber with a diameter of $14.46 \mu \mathrm{m}, \mathrm{C})$ PLGA micro-fiber with a diameter of $6.88 \mu \mathrm{m}$, and D) gelatin/PLGA (70:30) composite fiber with a diameter of $7.15 \mu \mathrm{m}$

To further demonstrate the direct-write technique has the ability to precisely control the micro-fibers' three-dimensional spatial position, freely suspended branched structures with different branching angles $\left(60^{\circ}, 120^{\circ} \text {, and } 150\right)^{\circ}$ were designed and fabricated on the acrylic frame. Figure 4.3A-C shows the fabricated gelatin branched structures with measured branching angles of $63^{\circ}, 116^{\circ}$, and $139^{\circ}$, respectively (processing factors: solution concentration $=16 \%$, Needle ID $=0.20$ $\mathrm{mm}$, feed rate $=100 \mathrm{~mm} / \mathrm{s}$ and fiber length $=8 \mathrm{~mm}$ ). The relative errors for the actual angles of these branched structures are $5.0 \%, 3.3 \%$, and $7.3 \%$, respectively. 
In section 2.1.1, we have learned that the capillary system consists of arterioles, capillaries, and venules, and those vessels have different diameter ranges. Thus, further developing a branched structure with varying diameters of micro-fiber could better mimic the real capillary system structure. One of the limitations of this 3-Axis robotic dispensing system is that the solution (i.e., concentration) and the needle cannot be changed during the fabrication process. However, the fiber diameter could still be controlled by varying the feed rate. Figure 4.3D shows a gelatin branched structure with a vertical supported fiber to mimic the arteriole (processing factors: solution concentration $=16 \%$, Needle ID $=$ $0.20 \mathrm{~mm}$, feed rate $=30 \mathrm{~mm} / \mathrm{s}$ ), whereas the branched fibers (Figure 4.3A-C) could work as the capillary. (remain other processing factors the same except for feed rate increase to $100 \mathrm{~mm} / \mathrm{s}$ ). Similarly, Figure $4.3 E-F$ shows branched structures with different fiber diameters fabricated from gelatin/PLGA composites (70:30) and PLGA, respectively. Another factor that would affect the fiber diameter is the fiber length. Generally, the branched fibers are longer than the supported fiber (i.e., two times longer for the $60^{\circ}$ branched structure) and have more touchdown point, which may cause extra stress and displace the supported fiber. Combined with different drawing speed, the different fiber diameters were achieved in one branched structure. 


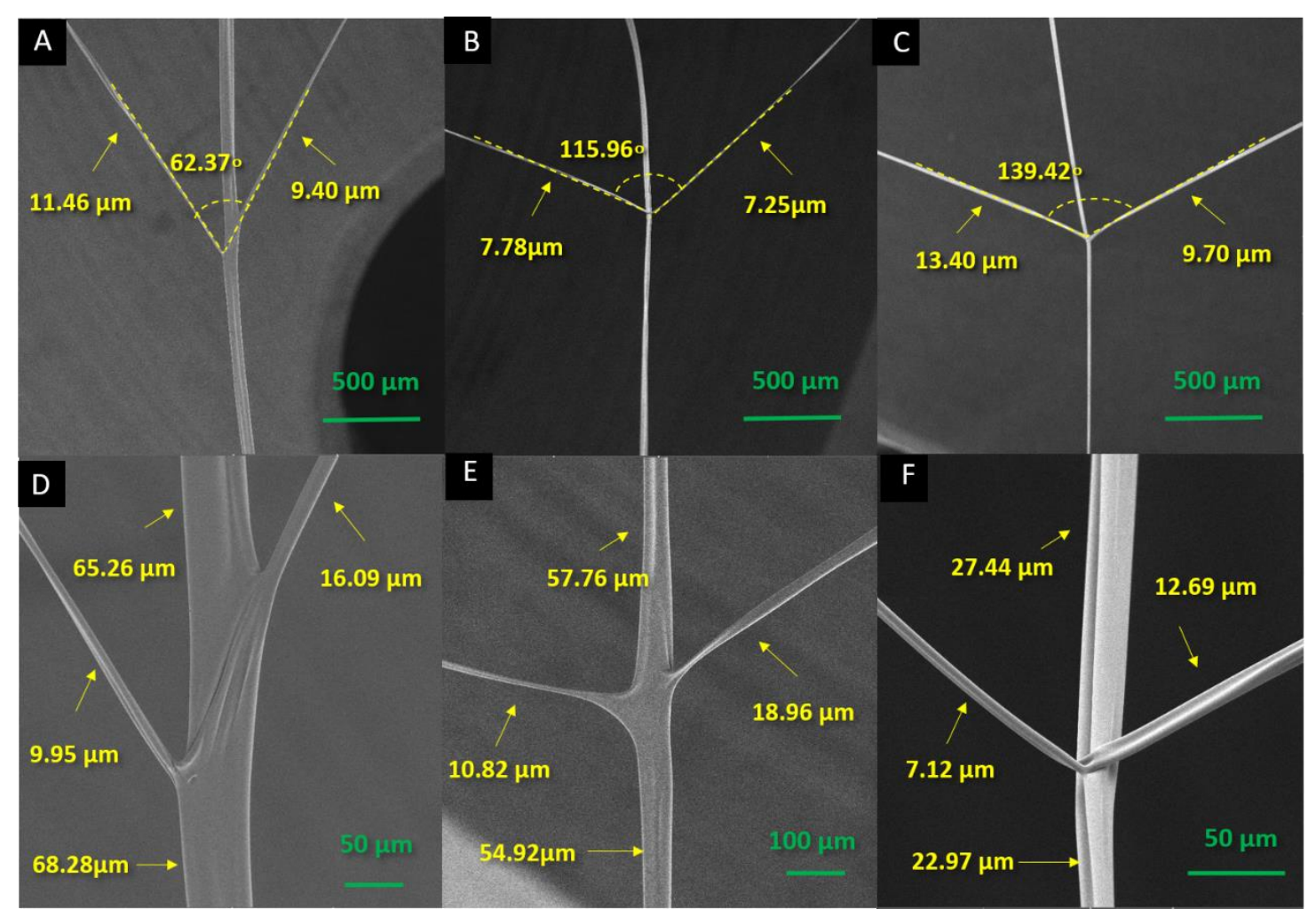

Figure 4. 3 SEM images of the gelatin branched structures with different branching angles: A) $62.37^{\circ}$, B) $115.96^{\circ}$, and C) $139.42^{\circ}$, and branched structures with different fiber diameters fabricated from: D) gelatin, E) gelatin/PLGA composites (70:30), and F) PLGA.

The infrared spectra of the pristine gelatin, PLGA, and their composites are presented in Figure 4.4. The characteristic absorption bands for $\mathrm{N}-\mathrm{H}$ stretching at $3291.78 \mathrm{~cm}^{-1}$, amide I, C-O and C-N stretching at $1636.66 \mathrm{~cm}^{-1}$, and amide II, N$\mathrm{H}$ in-plane bending and $\mathrm{C}-\mathrm{N}$ stretching at $1523.73 \mathrm{~cm}^{-1}$ could be found in the pristine gelatin and the different composites. The typical absorption bands for ester carbonyl stretching and vibration at $1749.91 \mathrm{~cm}^{-1}$ and C-O-C ether group stretching 
at $1084.34 \mathrm{~cm}^{-1}$ were also found in pure PLGA and the composites. Increasing the amount of PLGA caused the intensity of C-O stretching and C-O-C group stretching peaks to decrease. No new peak or peak shifting was observed in the composites. This suggests that there is no new bond formed or strong chemical interaction occurred within the composites, which confirms the gelatin/PLGA composites are just simply a physical mixture.

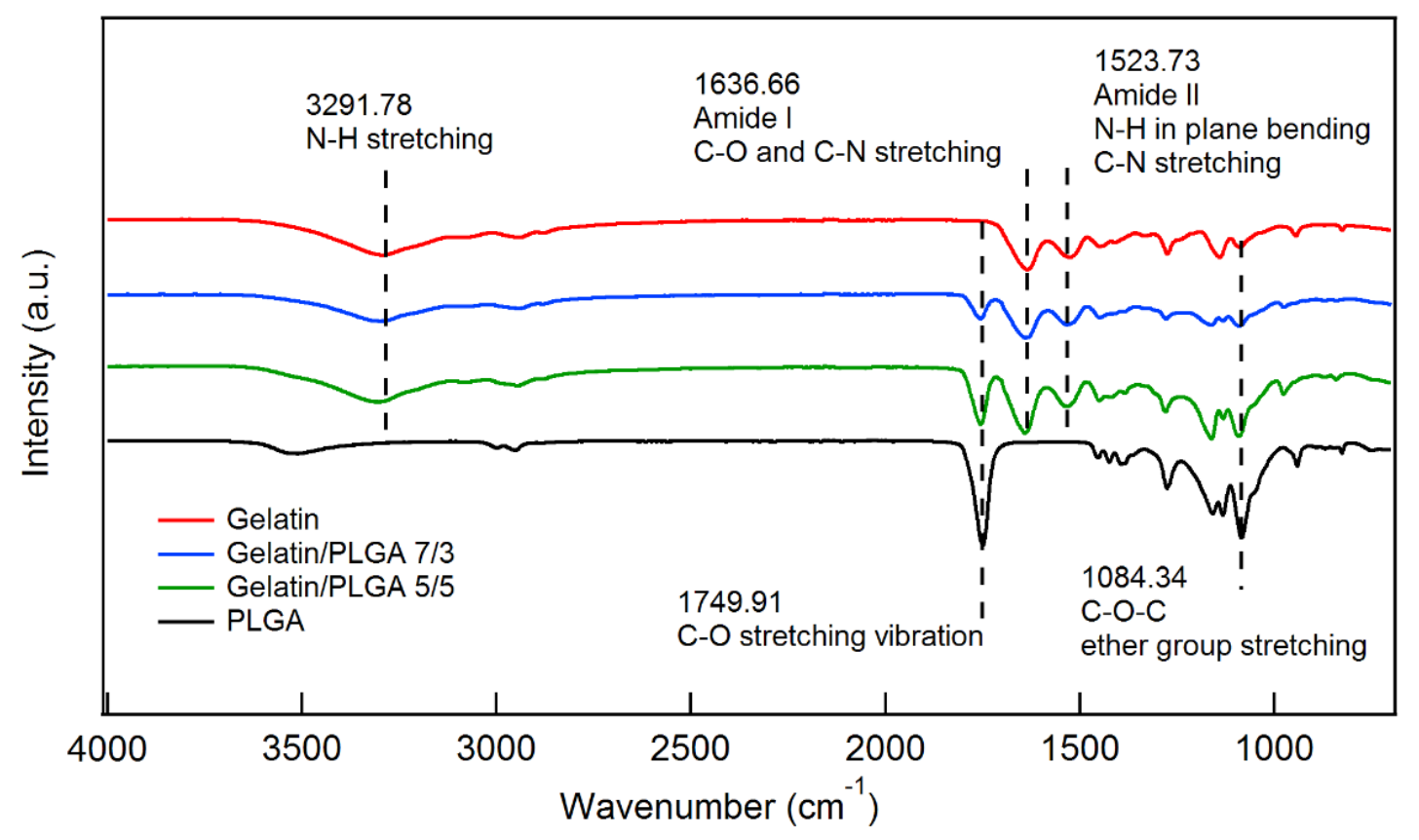

Figure 4. 4 FT-IR spectra of pristine gelatin, PLGA, and their composites

\subsubsection{PLA and PLA-PEG Copolymers}

Similarly, the optimal solvent selection for PLA and PLA-PEG copolymer was determined over several trials. Table 4.3 shows whether these polymer or 
copolymers are soluble in the common organic solvents at a concentration of at least $20 \%$ by weight.

Table 4. 3 Solvent trials for PLA and PLA-PEG copolymers

\begin{tabular}{|c|c|c|c|c|c|}
\hline Polymers & TFE & Chlorobenzene & $\begin{array}{c}1,2 \\
\text { Dichloroethane }\end{array}$ & Chloroform & Acetone \\
\hline PLA & $\bar{X}$ & $\bar{X}$ & $\checkmark$ & $\checkmark$ & $\bar{X}$ \\
\hline $\begin{array}{c}\text { PLA-PEG } \\
(90: 10)\end{array}$ & $X$ & $X$ & $\checkmark$ & $\checkmark$ & $x$ \\
\hline $\begin{array}{c}\text { PLA-PEG } \\
(75: 25)\end{array}$ & $X$ & $\mathrm{X}$ & $x$ & $\checkmark$ & $x$ \\
\hline $\begin{array}{c}\text { PLA-PEG } \\
(70: 30)\end{array}$ & $X$ & $X$ & $X$ & $\checkmark$ & $X$ \\
\hline $\begin{array}{l}\text { PLA-PEG } \\
(60: 40)\end{array}$ & $x$ & $X$ & $X$ & $\checkmark$ & $x$ \\
\hline $\begin{array}{l}\text { PLA-PEG } \\
(50: 50)\end{array}$ & $X$ & $X$ & $X$ & $\checkmark$ & $X$ \\
\hline
\end{tabular}

Chloroform was ultimately chosen based on the increased solubility of the polymers and moderate solvent volatility. Based on the preliminary experiments of copolymer degradation and fiber drawing ability, only PLA-PEG (75:25) and PLAPEG (70:30) were selected to draw the micro-fibers. PLA-PEG (75:25) solution was prepared by dissolving powder copolymer in chloroform of $20 \%$ and $21 \%$ by 
weight; PLA-PEG (70:30) solution was prepared by dissolving powder copolymer in chloroform of $30 \%$ and $31 \%$ by weight. PLA solutions were prepared by dissolving bead-shaped PLA in chloroform of $28 \%$ and $29 \%$ by weight (Table 4.4 ).

Table 4. 4 Selected concentration of PLA and PLA-PEG copolymer

\begin{tabular}{clc}
\hline Polymers & Solvent & Concentration \\
\hline PLA & Chloroform & $28 \%$ \\
PLA & Chloroform & $29 \%$ \\
PLA-PEG (75:25) & Chloroform & $20 \%$ \\
PLA-PEG (75:25) & Chloroform & $21 \%$ \\
PLA-PEG (70:30) & Chloroform & $30 \%$ \\
PLA-PEG (70:30) & Chloroform & $31 \%$ \\
\hline
\end{tabular}

PLA and the selected PLA-PEG copolymers solutions were successfully processed into suspended micro-fibers with a microvascular-scale diameter by direct-write technique. Figure 4.5A presents the single suspended PLA micro-fiber with a diameter of $15.47 \mu \mathrm{m}$ (processing factors: solution concentration $=28 \%$, needle ID $=0.25 \mathrm{~mm}$, feed rate $=100 \mathrm{~mm} / \mathrm{s}$, and fiber length $=6 \mathrm{~mm}$ ); Figure 4.5B shows the PLA-PEG (75:25) copolymer micro-fiber with a diameter of $9.05 \mu \mathrm{m}$ (processing factors: solution concentration $=21 \%$, needle ID $=0.25 \mathrm{~mm}$, feed rate $=100 \mathrm{~mm} / \mathrm{s}$, and fiber length $=8 \mathrm{~mm}$ ); Figure $4.5 \mathrm{C}$ demonstrates the PLA-PEG (70:30) micro-fibers with a diameter of $6.52 \mu \mathrm{m}$ (processing factors: solution concentration $=31 \%$, needle ID $=0.25 \mathrm{~mm}$, feed rate $=100 \mathrm{~mm} / \mathrm{s}$, and fiber length 
$=10 \mathrm{~mm}$ ). The SEM images indicate the produced micro-fibers have a smooth surface and no bead was observed for most of the surface.

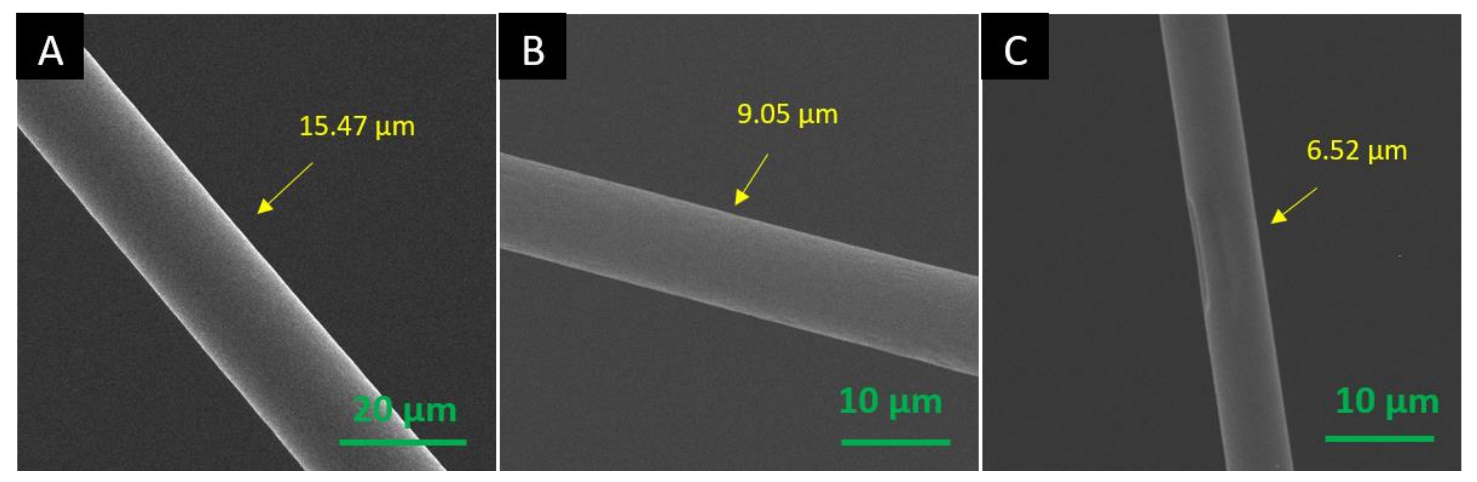

Figure 4. 5 SEM images of representative single suspended micro-fibers fabricated by the 3-Axis robotic dispensing system. A) PLA fiber with the diameter of 15.47 $\mu \mathrm{m}$, B) PLA-PEG (75:25) fiber with the diameter of $9.05 \mu \mathrm{m}$, and C) PLA-PEG (70:30) fiber with the diameter of $6.52 \mu \mathrm{m}$.

PLA and PLA-PEG copolymer were also used to produce suspended branched structures. Figure 4.6A-B shows PLA and PLA-PEG (75:25) copolymer branched structures both with a vertical supported fiber and the branched fibers, respectively (processing factors for PLA: solution concentration $=28 \%$, needle ID $=0.25 \mathrm{~mm}$, feed rate $=100 \mathrm{~mm} / \mathrm{s}$; processing factors for PLA-PEG $(75: 25$ copolymer): solution concentration $=21 \%$, needle ID $=0.25 \mathrm{~mm}$, feed rate $=100$ $\mathrm{mm} / \mathrm{s}$. All supported fibers length $=8 \mathrm{~mm}$ ). 

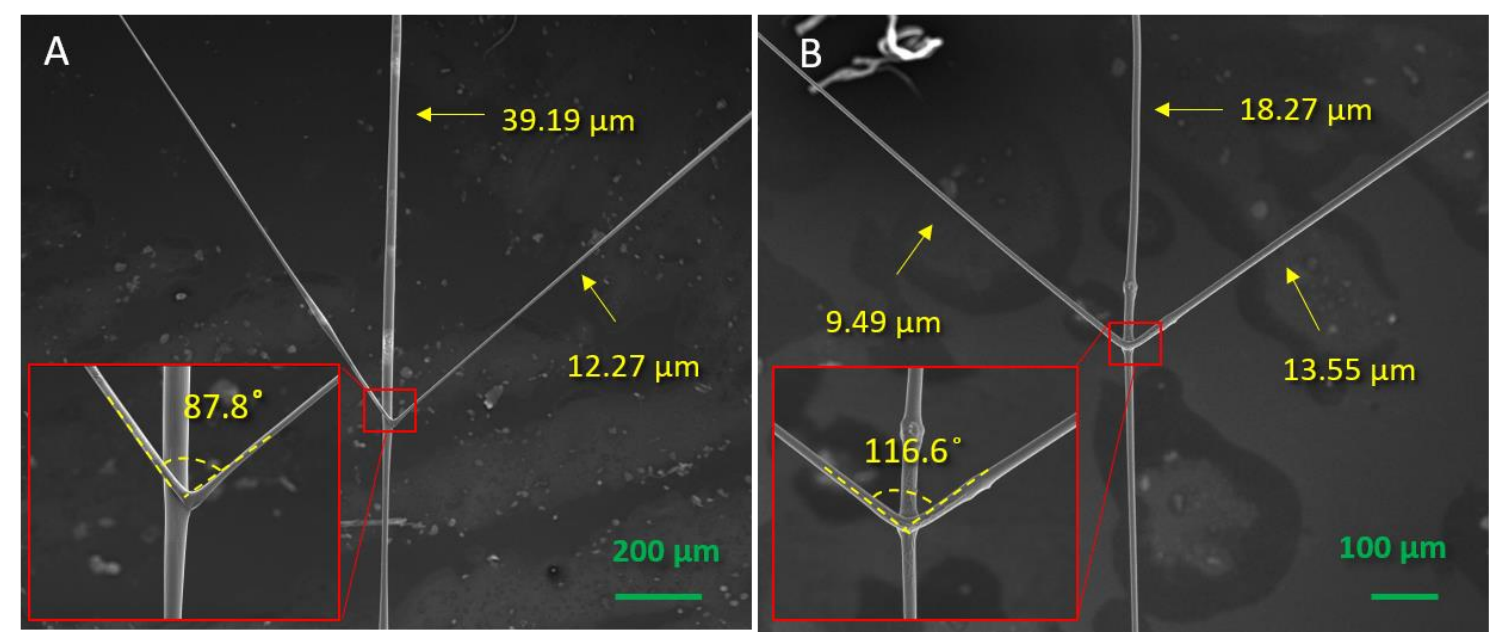

Figure 4. 6 SEM images of the representative branched structures fabricated by the direct-write technique from different biopolymers. A) PLA; B) PLA-PEG (75:25) copolymer. Inserted images showed the enlarged intersection area.

Besides producing single intersection branched structures, the 3-Axis robotic dispensing system is able to generate several complex structures by precisely controlling the dispensing tip to move among the predefined spatial spots, including the initiating, terminating, and intersection points. Figure 4.7A shows a web structure with triple intersections and all the branching angles designed to $90^{\circ}$. The actual angles for those three branched fibers are $88.9^{\circ}, 84.7^{\circ}$, and $100.3^{\circ}$, with a relative error of $0.9 \%, 4.4 \%$, and $8.6 \%$, respectively. The relative error increased with the drawing order; the probable reason is that the tension induced from the previous fiber may move the supported fiber and lead the displacement of the following fiber. Figure 4.7B demonstrates a dual-intersections branched structure with different branching angles (designed to $60^{\circ}$ and 120 ). The measured angles are $59.2^{\circ}$ and $113.9^{\circ}$, with a relative error of $1.3 \%$ and $5.1 \%$, respectively. Figure 
4.7C-D shows the parallel fiber arrays and overlapping fibers with orthotropic structure, in which all fibers in those structures are within the microvascular-scale diameters. Although those two structures are not similar to the real capillary system, they still have the potential application value such as to be used to study the angiogenesis behaviors of the endothelial cells between neighboring vessels.
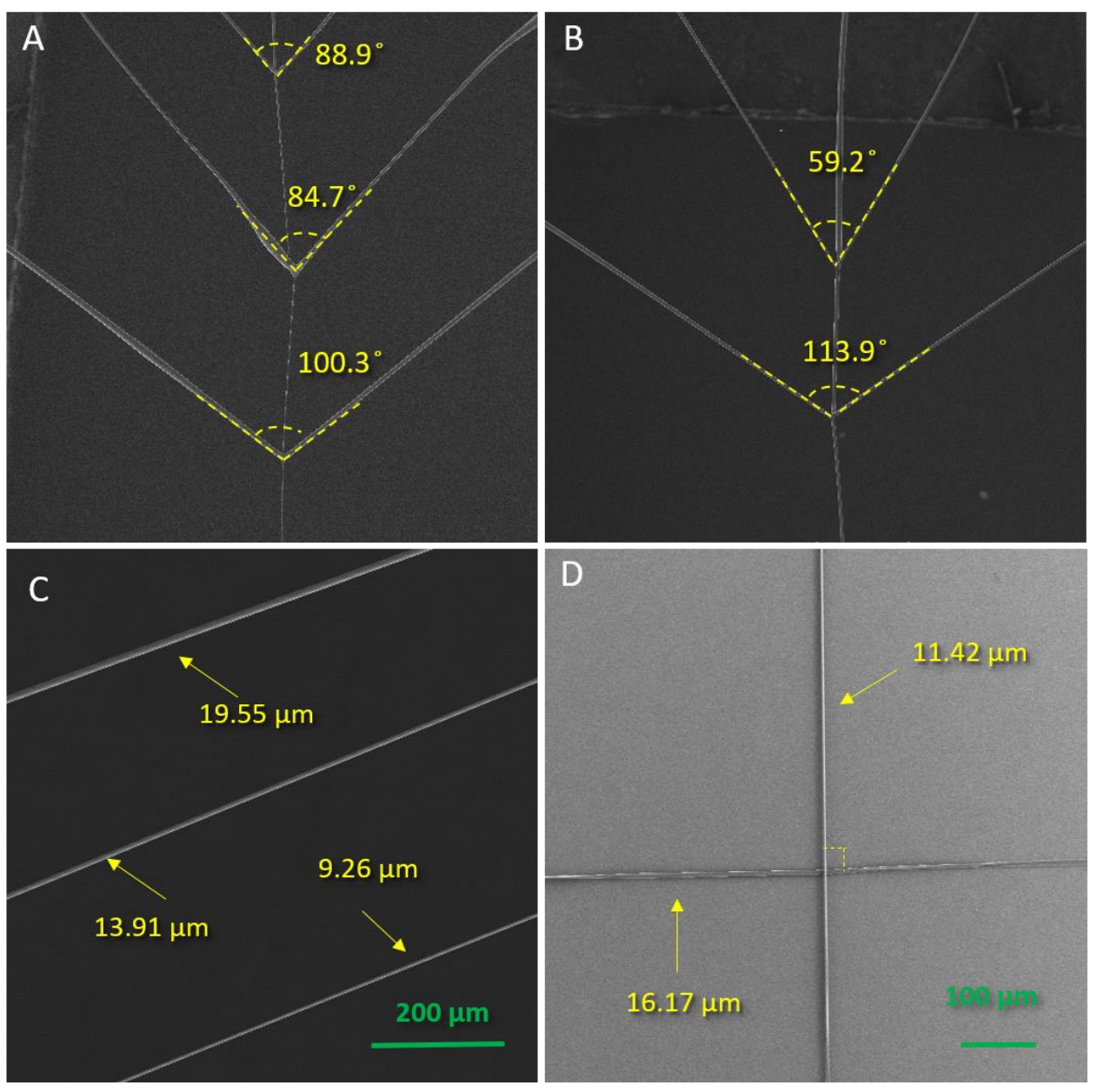

Figure 4. 7 SEM images of complex suspend structures fabricated from PLA-PEG (75:25) copolymer by direct-write technique. A) A branched structure with triple intersections; B) a dual-intersections branched structure; C) parallel fibers array; and D) overlapping fibers with orthotropic structure. 
The pristine PLA, PEG, and PLA-PEG copolymers were characterized by using FT-IR and ${ }^{1} \mathrm{H}-\mathrm{NMR}$. The infrared spectrum is presented in Figure 4.8. A strong absorption peak at $1746.77 \mathrm{~cm}^{-1}$ was observed in the PLA, which confirmed the presence of the ester stretching. Meanwhile, the FT-IR spectrum of PEG and the copolymers showed characteristic peaks at $2887.45 \mathrm{~cm}^{-1}$ and $3458.25 \mathrm{~cm}^{-1}$, which correspond to $\mathrm{C}-\mathrm{H}$ stretching and terminal hydroxyl group $\mathrm{O}-\mathrm{H}$ stretching, respectively. For the series of copolymers, the ester stretching peaks could be seen at $1757.88-1759.32 \mathrm{~cm}^{-1}$. The peaks shifting indicate the formation of the copolymers. The $\mathrm{C}-\mathrm{H}$ stretching and $\mathrm{O}-\mathrm{H}$ stretching peaks were also observed in those copolymers between $2885.36-2888.12 \mathrm{~cm}^{-1}$ and $3456.11-3459.43 \mathrm{~cm}^{-1}$, respectively. The intensity reduction of these two peaks also suggests there may be a new bond formed from the weak chemical interaction between the PLA and PEG in the copolymer. 


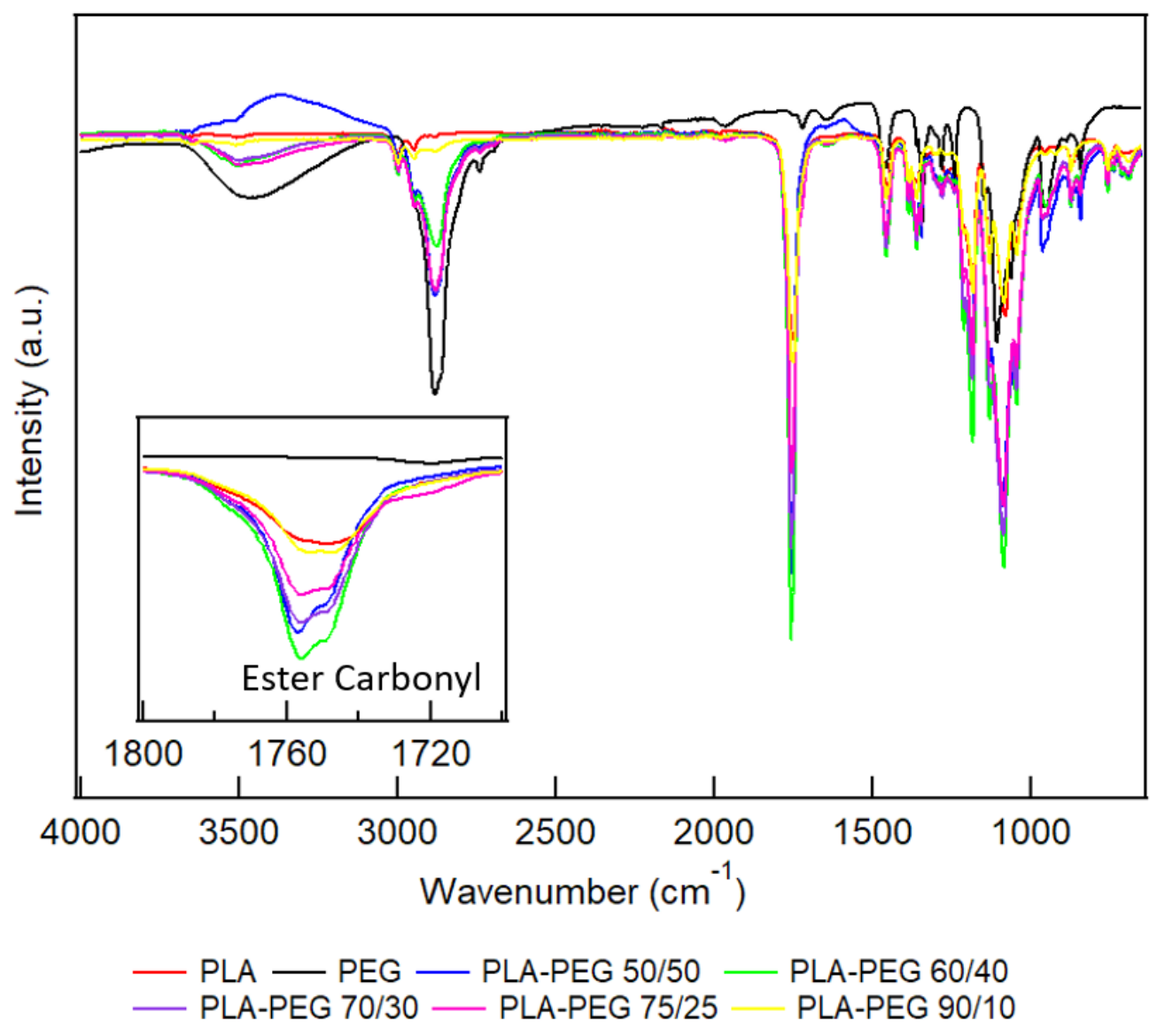

Figure 4. 8 FT-IR spectra of pristine PLA, PEG, and PLA-PEG copolymers

To further confirm the copolymer formation, the ${ }^{1} \mathrm{H}-\mathrm{NMR}$ spectra of pristine PLA, PEG, and PLA-PEG copolymers are also shown in Figure 4.9 (stacked images with $15^{\circ}$ tilt). For the PLA micro-fibers, the chemical shift at $5.15 \mathrm{ppm}$ and 1.52 ppm was observed, which corresponded to $\mathrm{CH}$ - and $\mathrm{CH}_{3}$ - bond, respectively. The calculated integration ratio for these two peaks is $1: 3.1$, which confirms the two types of hydrogen protons found in the chemical structure of PLA. For the PEG micro-fibers, we found a single peak with a chemical shift at $3.63 \mathrm{ppm}$, which indicates the $\mathrm{CH}_{2}$ - bond. The series spectra of the copolymers show new peaks at 
4.29 ppm. We speculate those peaks corresponded to the $\mathrm{CH}_{2}-\mathrm{O}$ hydrogen protons from the newly formed ester bond (highlighted with the red circle in Figure 4.9). The new ester may be formed between the break of $C(O)-O$ bond from PLA and $\mathrm{CH}_{2}-\mathrm{OCH}_{2}$ bond from PEG. In general, the intensity of the new peaks shows a reduction trend with the decreasing concentration of $P E G$, which suggests incomplete copolymerization due to the lack of the $\mathrm{CH}_{2}-\mathrm{OCH}_{2}$ bond from PEG. We also performed a polymer molecule NMR simulation by ChemNMR ${ }^{1} \mathrm{H}$ Estimation (Figure 4.10). For the given copolymer molecule with an ester group, a chemical shift appears at $4.27 \mathrm{ppm}$; this trend is observed in our actual ${ }^{1} \mathrm{H}-\mathrm{NMR}$ spectra of those copolymers. 


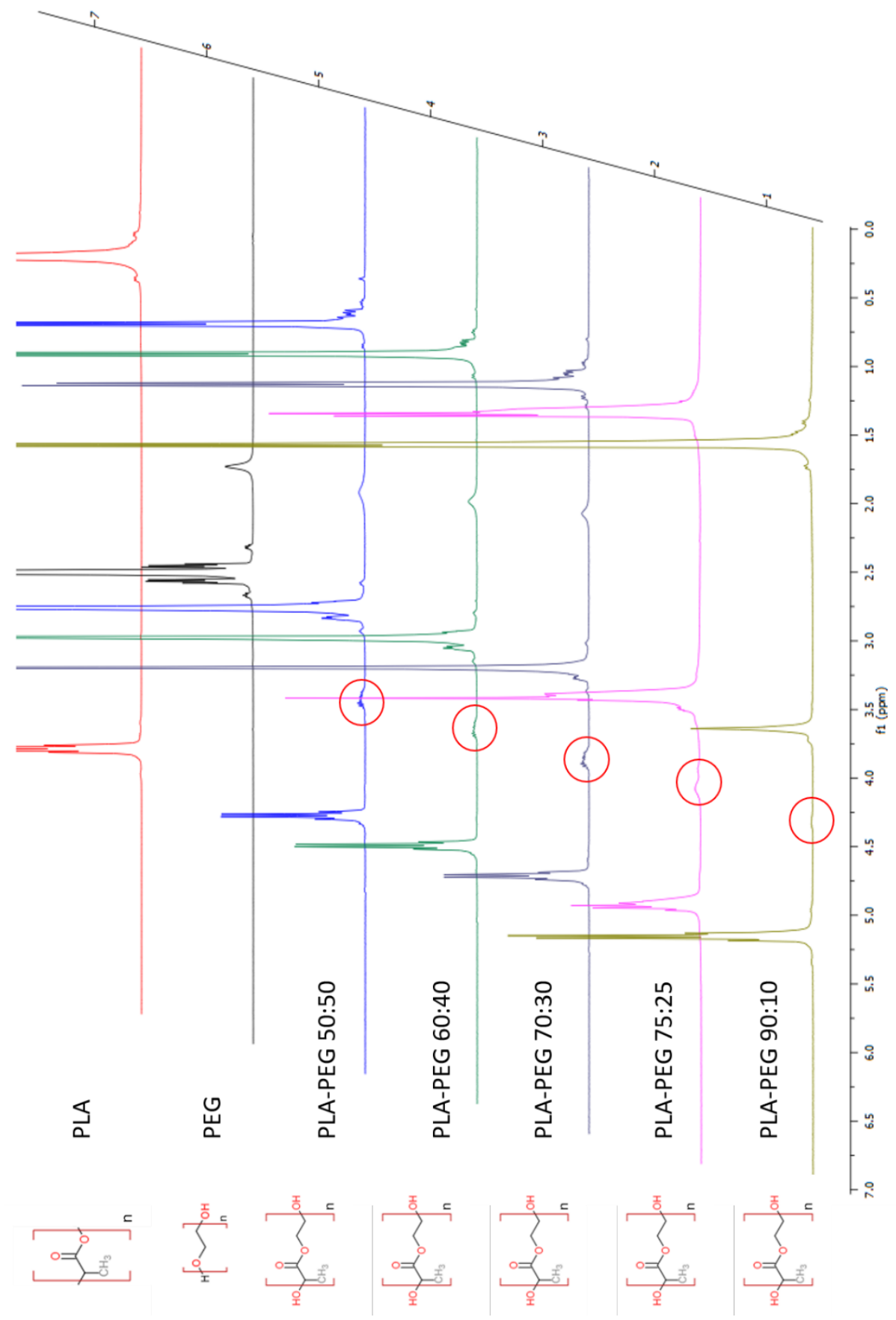

Figure 4. $9{ }^{1} \mathrm{H}-\mathrm{NMR}$ spectra of PLA, PEG, and PLA-PEG copolymer 


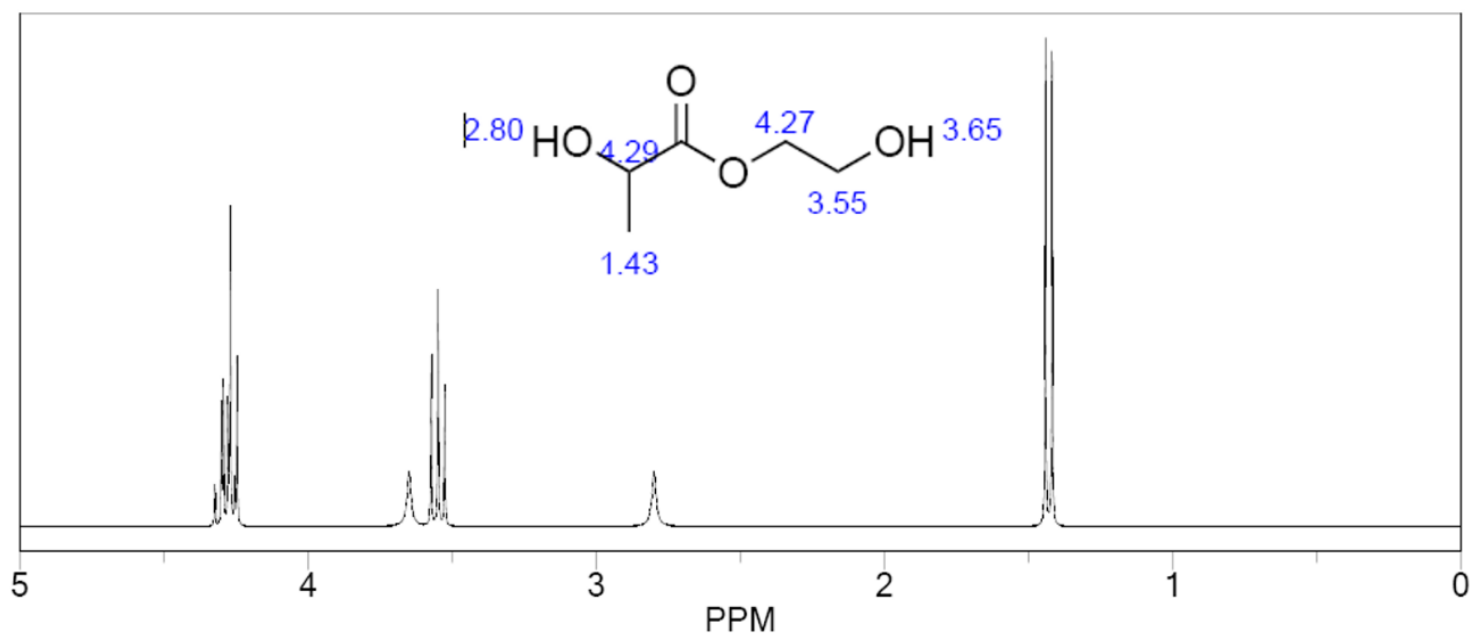

Figure 4. 10 ChemNMR ${ }^{1} \mathrm{H}$ Estimation of the formed copolymer

\subsection{Characterization and Modeling of the Direct-write Process}

We have demonstrated the ability to process a variety of biopolymers into micro-fibers and branched structures with microvascular-scale diameters by the direct-write technique using the 3-Axis robotic dispensing system. In order to better understand the micro-fiber formation mechanism behind the thinning dynamics of biopolymer solutions, the direct-write process needed to be characterized so that we can find out what process factors affected the micro-fiber yield and diameter. An empirical model generated from the characterization process could reveal the correlation among the fiber diameter to polymer solution properties and system process parameters. The empirical model also may offer future users the ability to employ the 3-Axis robotic dispensing system to direct-write micro-fibers without trial-and-error work. The empirical model was validated by comparing the 
prediction and experimental value of the diameters of the micro-fibers of various biopolymers other than gelatin.

\subsubsection{Characterization of the Direct-write Process}

\subsubsection{Process Factors Reduction}

There are eleven controllable variables involved in the direct-write process (Figure 4.11). The factors highlighted in orange are the solution physical properties, whereas those highlighted in blue are the process factors defined by the 3-Axis robotic dispensing system. Investigating all of these variables at once would result in a model with a large amount of uncertainty; thus, some variables must be set as constants. Based on the direct-write experiences from our group, valve pressure (15 psi) and dispensing time (0.2 seconds) were set as constant for the maximum performance of this system.

\begin{tabular}{|c|}
\hline Concentration \\
\hline Viscosity \\
\hline Surface tension \\
\hline Evaporation rate \\
\hline
\end{tabular}

\begin{tabular}{|c|}
\hline Needle/Substrate gap \\
\hline Valve time \\
\hline Valve pressure \\
\hline Dispensing time \\
\hline
\end{tabular}

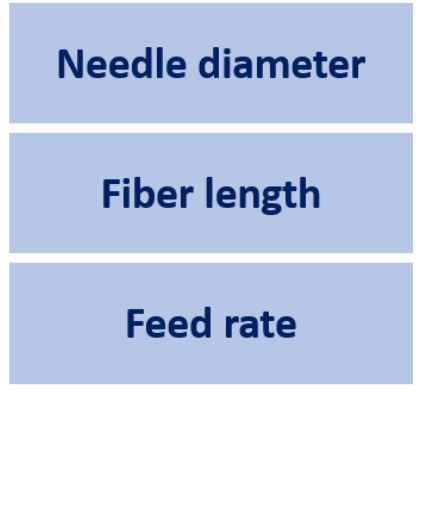

Figure 4. 11 Controllable factors involved in the direct-write process 
Before the fiber was drawn, the polymer drop's size ( $\left.D_{0}\right)$ is critical because it could be used to define the needle/substrate gap (Figure 3.28) and calculate the aspect ratio $(\Lambda)$ for dimensional analysis (Equation 3-11). Figure 4.12 and Table 4.5 present the $D_{0}$ measurements of the $16 \%$ gelatin solution with different needles $(0.25 \mathrm{~mm}, 0.20 \mathrm{~mm}, 0.15 \mathrm{~mm}$, and $0.10 \mathrm{~mm})$ and valve open time $(0.02 \mathrm{~s}, 0.05 \mathrm{~s}$, $0.1 \mathrm{~s}$, and $0.5 \mathrm{~s})$ under constant valve pressure (15 psi). Valve open time (0.1 second) was selected for the future drawing to reduce the system complexity and further define the needle/substrate gap.

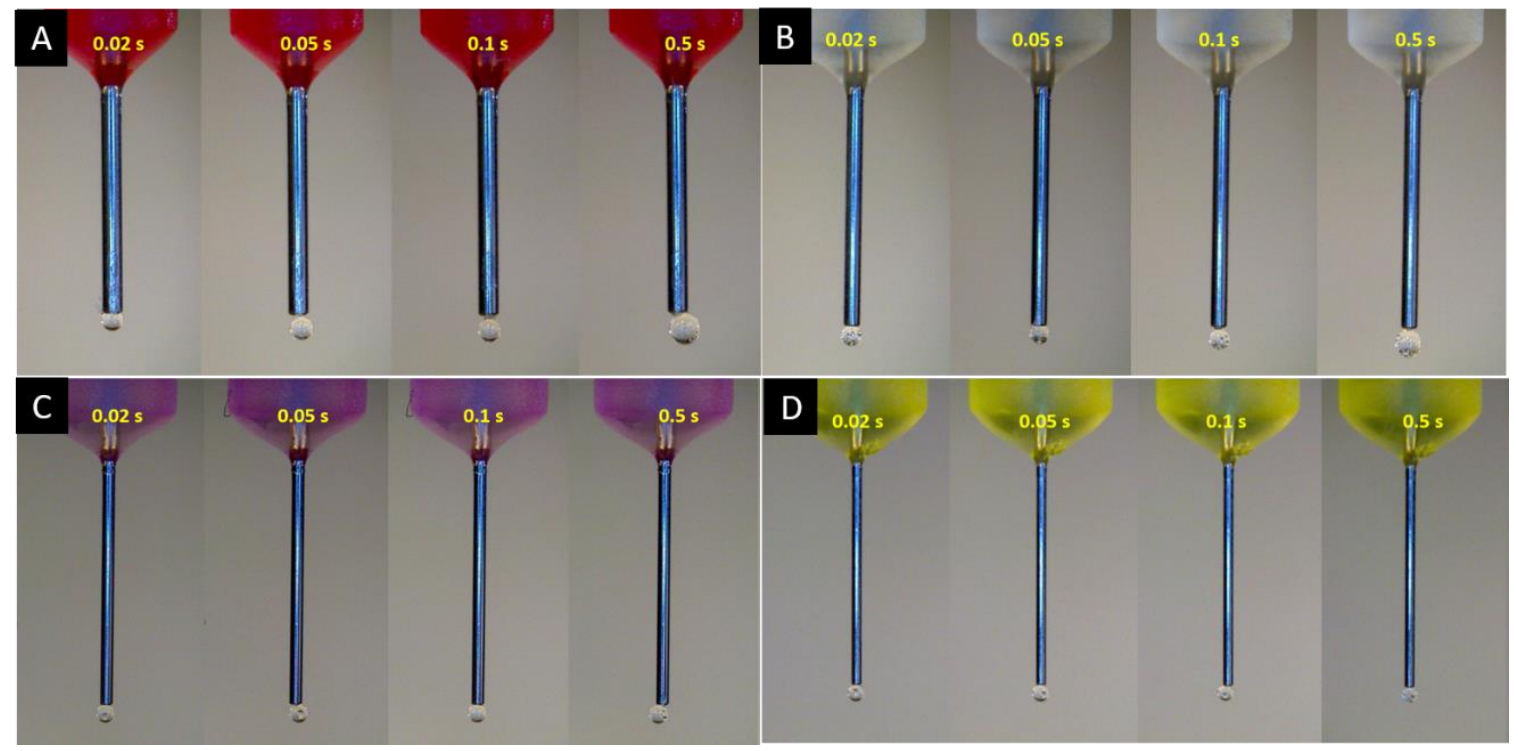

Figure 4. 12 Optical images of the gelatin solution drops with different needles and valve open time 
Table 4. 5 Gelatin polymer drop diameter measurements

\begin{tabular}{c|cccc}
\hline $\begin{array}{c}\text { Valve Open } \\
\text { time (s) }\end{array}$ & \multicolumn{4}{|c}{ Polymer Drop Diameter $(\boldsymbol{\mu m})$} \\
\cline { 2 - 5 } Needle ID (mm) & $\mathbf{0 . 0 2 ~ \mathbf { s }}$ & $\mathbf{0 . 0 5 ~ \mathbf { s }}$ & $\mathbf{0 . 1} \mathbf{~ s}$ & $\mathbf{0 . 5 ~ s}$ \\
\hline $\mathbf{0 . 2 5}$ (red) & $460.9 \pm 15.1$ & $482.7 \pm 4.3$ & $548.1 \pm 7.9$ & $622.0 \pm 11.3$ \\
$\mathbf{0 . 2 0}$ (clear) & $433.0 \pm 3.8$ & $454.2 \pm 10.6$ & $484.4 \pm 13.9$ & $539.6 \pm 9.8$ \\
$\mathbf{0 . 1 0}$ (lavender) & $391.9 \pm 7.1$ & $417.8 \pm 13.5$ & $435.5 \pm 7.2$ & $444.4 \pm 18.8$ \\
$\mathbf{0 . 1 0}$ (yellow) & $365.8 \pm 12.8$ & $404.0 \pm 15.3$ & $371.8 \pm 12.3$ & $394.1 \pm 12.9$ \\
\hline
\end{tabular}

In Figure 4.13, it is demonstrated how the needle/substrate gap could affect the contact between the polymer droplet and the substrate. When the gap was too small (second images for each needle), it was found that the polymer droplet would cause fouling outside of the needle. The fouling causes difficulty in producing a continuous direct-write series of fibers. If the gap was too big (fourth images for each needle), most of the fiber failed because the minimal contact reduces the adhesion between the polymer droplet and the substrate. The ideal needle/substrate gap was shown in the third images for each needle: $400 \mu \mathrm{m}$ for the red needle, $300 \mu \mathrm{m}$ for the clear needle, $250 \mu \mathrm{m}$ for the lavender needle, and $200 \mu \mathrm{m}$ for the yellow needle. 


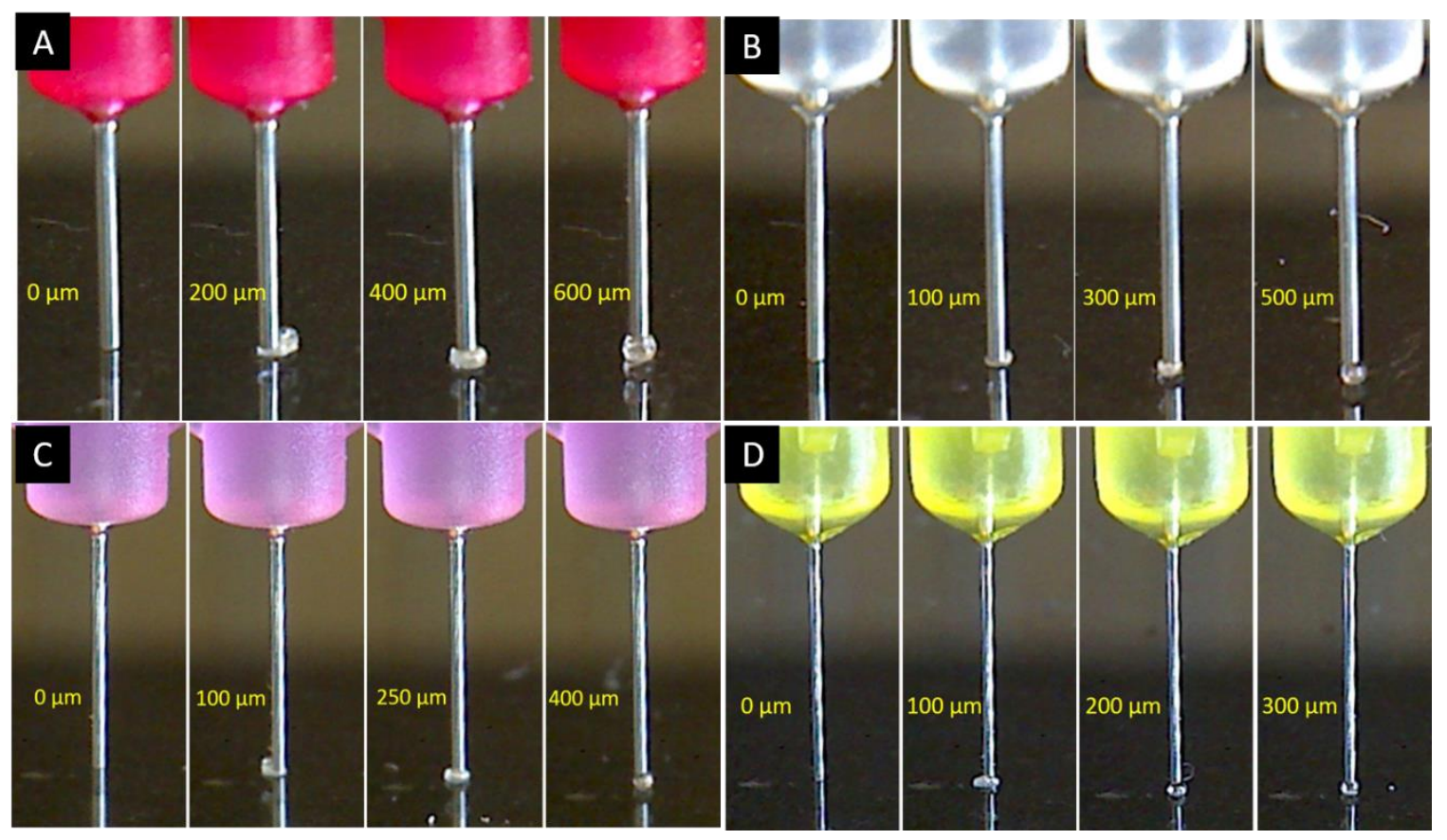

Figure 4. 13 Optical images of direct-write polymer droplet with differing needle/substrate distances across different needle inner diameters. A) $0.25 \mathrm{~mm}$, B) $0.20 \mathrm{~mm}$, C) $0.15 \mathrm{~mm}$, and D) $0.10 \mathrm{~mm}$

\subsubsection{Design of Experiments}

After reducing the number of variables, the significance of the physical properties of the solution and machine process factors were investigated by utilizing an unbalanced four factors multi-level full factorial design of experiment. Arrays of gelatin micro-fibers were fabricated on the substrate using the 3-Axis robotic dispensing system from different combinations. Figure 4.14 presents an example of how to measure the fiber diameter through the SEM. The diameters of each fiber were measured and recorded at the middle point as well as $200 \mu \mathrm{m}$ from the initiating and terminating side, and the average value was used as the final 
fiber diameter. Examples of gelatin fibers in different diameters range are shown in Figure 4.15.

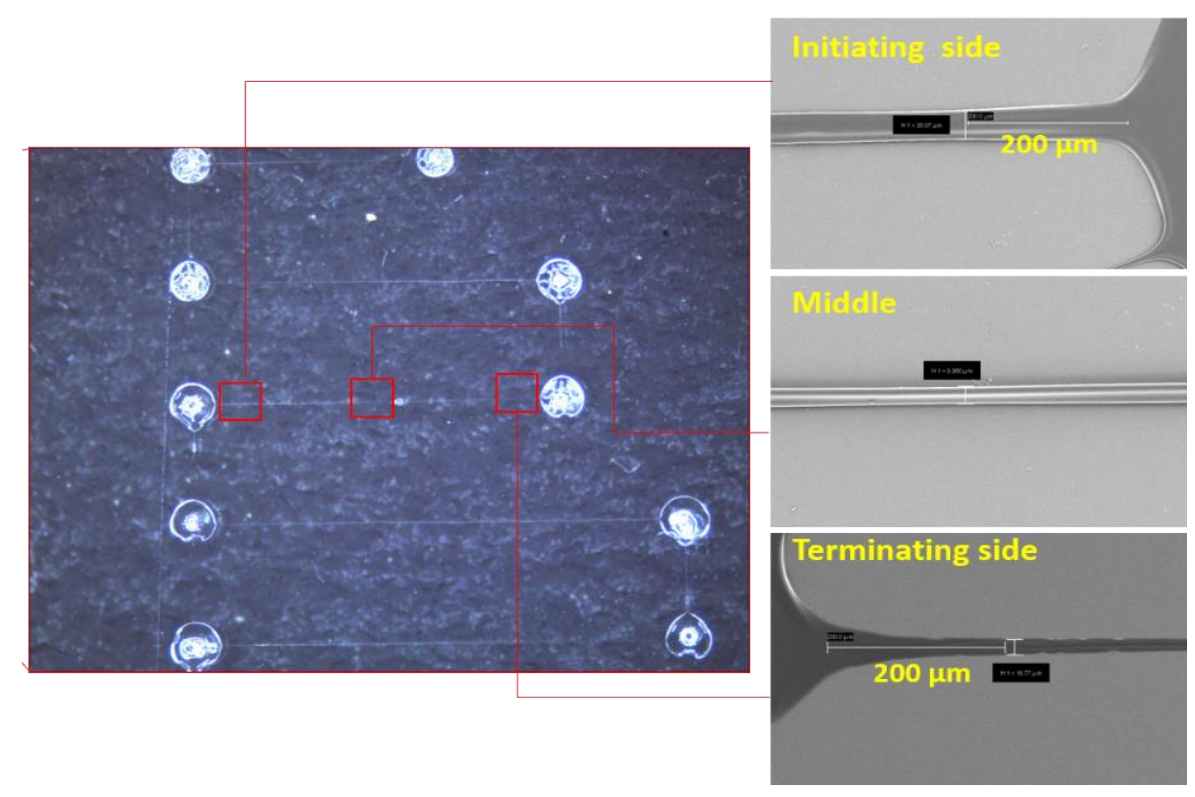

Figure 4. 14 Example of measuring the diameter of the micro-fiber from the fibers array by the SEM.

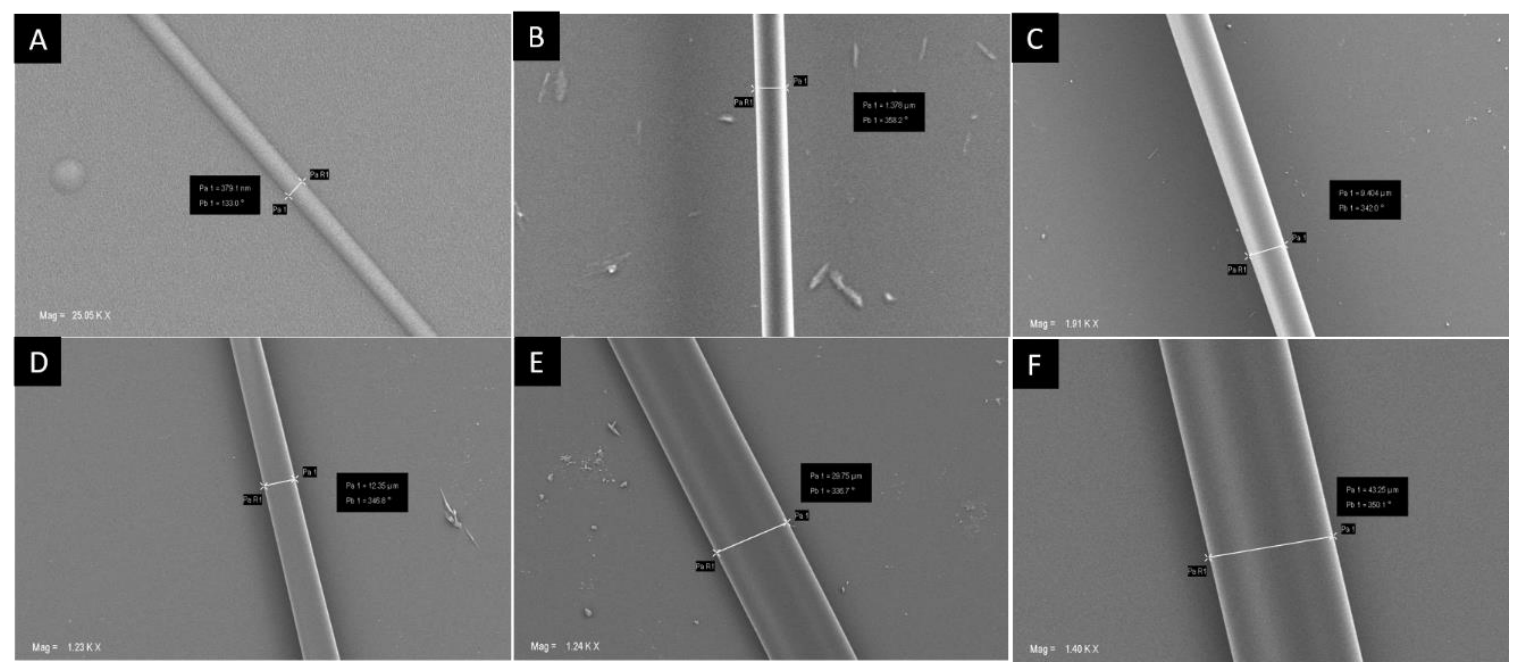

Figure 4. 15 SEM images of gelatin fibers with different fiber diameters from the DOE: A) $<1 \mu \mathrm{m}$, B) $1-5 \mu \mathrm{m}$, C) 5-10 $\mu \mathrm{m}$, D) 10-20 $\mu \mathrm{m}$, E) $20-30 \mu \mathrm{m}$, and F) $>40$ $\mu \mathrm{m}$ 
Statistical analysis of the experimental data has been done using an ANOVA. ANOVA enables us to gain insight into the direct-write process by distinguishing which factors have significant effects on the mean fiber diameter. The ANOVA table for fiber diameters is shown in Table 4.6. The "main effects" plots of the mean fiber diameter as a function of four different factors are presented in Figure 4.16. We can see that all four selected factors are significant for fiber diameters $(p=0.000)$. Generally, the mean fiber diameters increased with increasing solution concentration and the needle's inner diameters and decreasing feed rate and fiber length. The solution concentration could further affect three measurable physical properties: viscosity, surface tension, and mass transfer coefficient; thus, the more accurate fiber diameter correlation will be illustrated in the empirical model section later.

Table 4. 6 The ANOVA table for fiber diameters

Analysis of Variance for Transformed Response
\begin{tabular}{lrrrrr} 
Source & DF & Adj SS & Adj MS & F-Value & P-Value \\
\hline Concentration (wt\%) & 2 & 380.578 & 190.289 & 1609.72 & 0.000 \\
Needle ID (mm) & 3 & 3.206 & 1.069 & 9.04 & 0.000 \\
Feed rate (mm/s) & 2 & 5.243 & 2.622 & 22.18 & 0.000 \\
Fiber length (mm) & 11 & 144.911 & 13.174 & 111.44 & 0.000 \\
Error & 614 & 72.582 & 0.118 & & \\
Lack-of-Fit & 340 & 57.806 & 0.170 & 3.15 & 0.000 \\
$\quad$ Pure Error & 274 & 14.776 & 0.054 & & \\
Total & 632 & 578.842 & & &
\end{tabular}




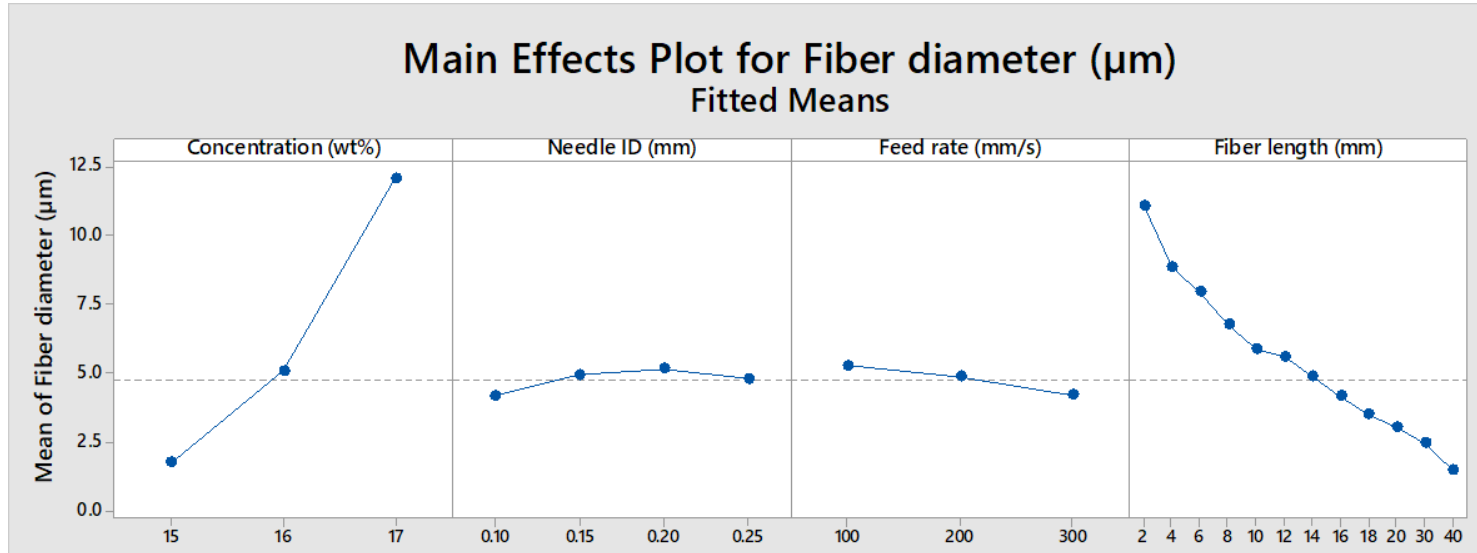

Figure 4. 16 The "main effects" plots of the mean of fiber diameter as a function of concentration (\%), needle ID $(\mathrm{mm})$, feed rate $(\mathrm{mm} / \mathrm{s})$, and fiber length $(\mathrm{mm})$

The effects of these four factors on the fiber yield are presented in Figure 4.17. Figure $4.17 \mathrm{~A}$ suggests the $16 \%$ gelatin solution has a higher yield $(\sim 79 \%)$ compared to $15 \%$ and $17 \%$. Figure 4.17B shows that the fiber yield increased with the increasing needle's inner diameter due to the enlarged initial polymer droplet diameter $\left(D_{0}\right)$. The feed rate also significantly affects the fiber yield (Figure 4.17C). Fiber drawn at $200 \mathrm{~mm} / \mathrm{s}$ shows the highest yield ( 77\%), while feed rates that are too slow or too fast will both decrease the fiber yield. Figure 4.17D illustrates the relationship between the fiber yield and the fiber length. This general trend suggests that at a shorter length $(<8 \mathrm{~mm})$, the fiber yield remained at a high level (>88\%). However, as the fiber length increased, the fiber yield dropped dramatically. 


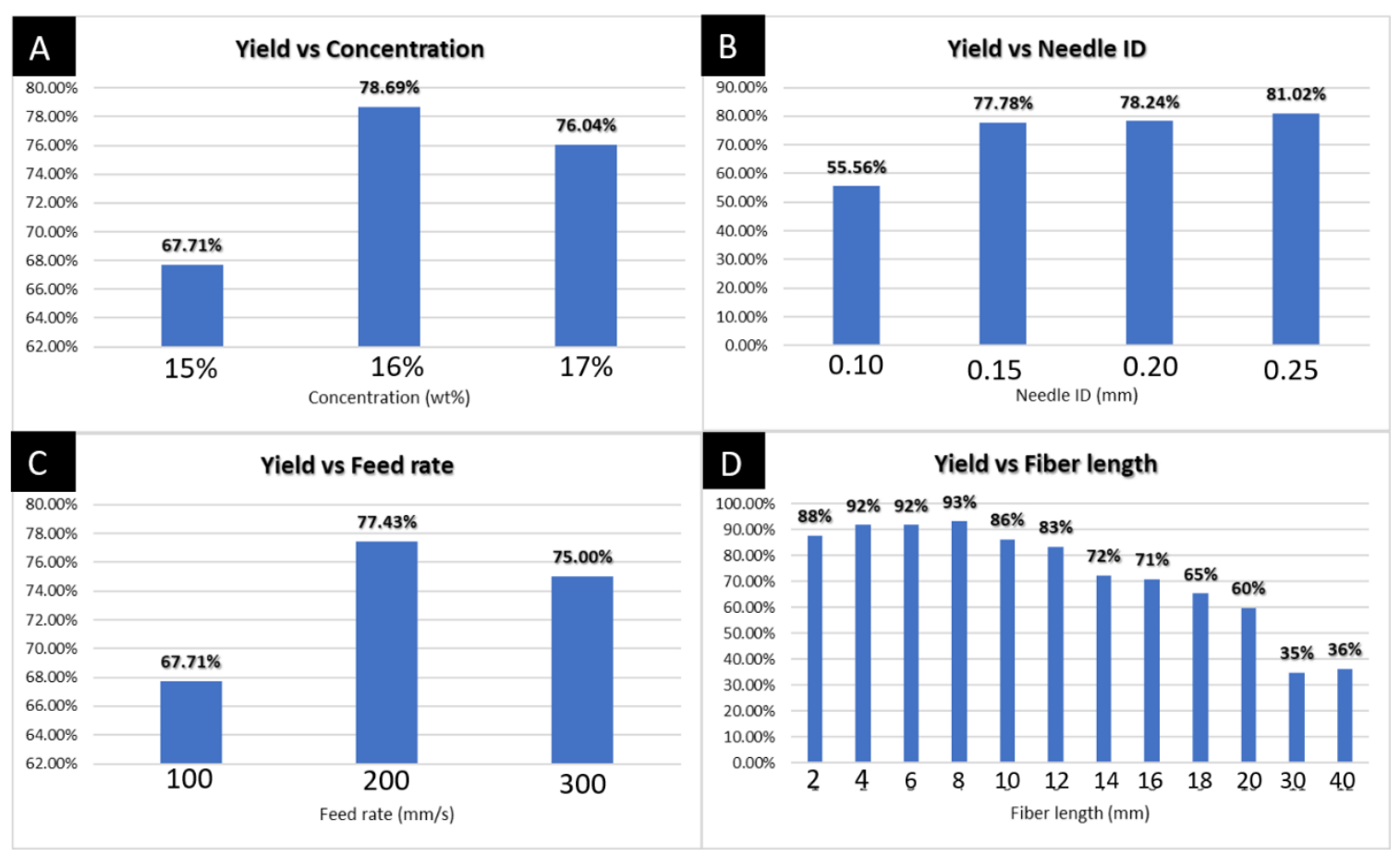

Figure 4. 17 Yield of direct-writing gelatin fibers versus several factors: A) concentration (\%), B) needle inner diameter $(\mathrm{mm})$, C) feed rate $(\mathrm{mm} / \mathrm{s})$, and $\mathrm{D})$ fiber length (mm).

The DOE indicates that several requirements need to be met to fabricate micro-fibers successfully with the direct-write method. First, rheological properties of the solution should be appropriately examined. During the drawing process, the appropriate choice in solution concentration and needle size ensures that the solution has sufficient surface tension to achieve the necking effect, enough viscosity to resist capillary breakup, and proper volatility to promote solidification of the fiber. Second, the drawing process factors also should be finely tuned. The proper workable feed rate and fiber length ensures either the drawing velocity is not too fast to avoid uncompleted surface tension-driven thinning and the fiber is not too long to cause excessive thinning, resulting in probable breakup. 


\subsubsection{Generation of the Empirical Model}

\subsubsection{Characterization of Gelatin Solution}

The physical properties of the gelatin solutions are summarized in Table 4.7. The one-way ANOVA results illustrate both the viscosity and surface tension of the gelatin solution significantly increase with increasing gelation concentration $(p<0.001)$. The mass transfer coefficient is independent of concentration due to the same solvent and solution/air interface area.

Table 4. 7 Physical properties of the gelatin solutions

\begin{tabular}{cccc}
\hline $\begin{array}{c}\text { Concentration } \\
(\mathbf{w t} . \%)\end{array}$ & $\begin{array}{c}\text { Viscosity, } \\
\boldsymbol{\eta}\left(\mathbf{P a}^{*} \mathbf{s}\right)\end{array}$ & $\begin{array}{c}\text { Surface tension, } \\
\boldsymbol{\sigma}(\mathbf{m N} / \mathbf{m})\end{array}$ & $\begin{array}{c}\text { Mass transfer } \\
\text { coef., } \mathbf{X}(\mathbf{m} / \mathbf{s})\end{array}$ \\
\hline 15 & $0.93 \pm 0.08$ & $27.52 \pm 0.46$ & $2.36 \mathrm{E}-07$ \\
16 & $1.12 \pm 0.11$ & $30.50 \pm 1.22$ & $2.36 \mathrm{E}-07$ \\
17 & $1.70 \pm 0.04$ & $39.18 \pm 0.80$ & $2.36 \mathrm{E}-07$ \\
\hline
\end{tabular}

The gelatin on dry status is a long chain of polymer molecules, which coil and associate with each other through specific, cooperative, non-covalent junction zones. Strictly speaking, the solution should be a non-Newtonian fluid. However, Stainsby et al. firstly pointed out that a concentrated gelation solution behaved like a Newtonian fluid at a temperature above the gel point[203]. We could estimate the apparent shear rate at the needle wall by approximating the solution as Newtonian fluids and adopting the equation as below: 


$$
\gamma^{\prime}=\frac{4 Q}{\pi R^{3}}
$$

Where $\gamma^{\prime}$ is the apparent shear rate at the needle wall, $Q$ is the volumetric flow rate, and $R$ is the inner radius of the needle. Figure 4.18 shows the relationship between shear rate and viscosity for gelatin solutions with different concentrations. The viscosity remains constant with the various shear rate from $\sim 0.1$ to $\sim 200 \mathrm{~s}^{-1}$. During the direct write process, the typical flow rate was $\sim 0.68$ $\mathrm{mL} \mathrm{h}^{-1}$ to $\sim 3.70 \mathrm{~mL} \mathrm{~h}^{-1}$ and the calculated shear rates were approximately $\sim 15-83$ $\mathrm{s}^{-1}$ (the region between the red dotted lines). Based on the results, it was confirmed that the gelatin solutions conform to the assumption of Newtonian-like fluid during the direct-write process and, thus, the assumption could be used for dimensionless model generation.

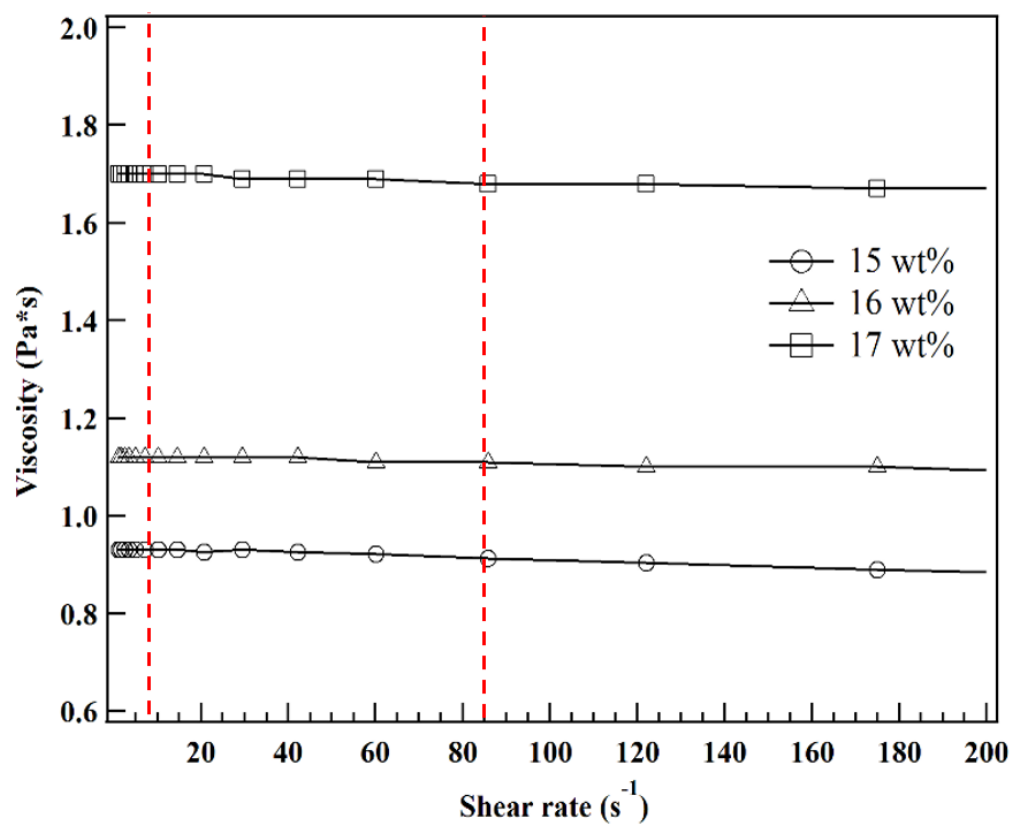


Figure 4. 18 The viscosity of gelatin solutions under different shear rates

\subsubsection{Dimensional Analysis for Empirical Model Generation}

The dimensional analysis is employed to obtain a certain set of information about a given physical issue. Through the dimensional analysis, we can reduce the multifaceted nature of a physical problem by removing variables that have limited influence on the given issue. In section 3.3.3.1, we introduced the Buckingham $\mathrm{Pi}$ theorem and showed how to group six parameters into three dimensionless parameters by selecting MLt (mass, length, and time) dimensions. The factors, symbols, and dimensions of the process factors used for the development of the empirical model are shown in Table 4.8. The dimensionless parameters $\mathrm{P}$ and $\mathrm{C}_{a}$ (Table 4.9) were calculated from the polymer solution physical properties in Table 4.7 by using Equations 3-7 and 3-10. The Aspect ratio $(\Lambda)$ was calculated from the polymer droplet diameter in Table 4.5 by using Equation 3-11. The values range from 7.30-72.99 based on the different fiber lengths $(2-40 \mathrm{~mm})$. 
Table 4. 8 Factors, symbols, and dimensions of the response, process factors, and the dimensionless parameters

\begin{tabular}{lccc}
\hline & Factors & Symbol & Dimensions \\
\hline Response & Fiber diameters & $\mathrm{D}_{\infty}$ & {$[\mathrm{L}]$} \\
\hline Viscosity & $\eta$ & {$\left[\mathrm{ML}^{-1} \mathrm{~T}^{-1}\right]$} \\
Process & Surface tension & $\sigma$ & {$\left[\mathrm{MT}^{-2}\right]$} \\
Factors & Mass transfer coefficient & $X$ & {$\left[\mathrm{LT}^{-1}\right]$} \\
& Feed rate & $\mathrm{U}$ & {$\left[\mathrm{LT}^{-1}\right]$} \\
& Fiber length & $\mathrm{L}$ & {$\left[\mathrm{L}^{-1}\right]$} \\
Dimensionless & Processability parameter & $\mathrm{P}$ & - \\
Parameters & Capillary Number & $\mathrm{Ca}$ & - \\
& Aspect ratio & $\wedge$ & - \\
\hline
\end{tabular}

Table 4. 9 Calculated dimensionless parameters of the gelatin solutions

\begin{tabular}{|c|c|c|c|c|}
\hline $\begin{array}{c}\text { Concentration } \\
\text { (wt.\%) }\end{array}$ & $\mathbf{P}$ & $\begin{array}{c}\mathrm{C} a @ \\
100 \mathrm{~mm} / \mathrm{s}\end{array}$ & $\begin{array}{c}\mathrm{Ca} @ \\
200 \mathrm{~mm} / \mathrm{s}\end{array}$ & $\begin{array}{c}C_{a} @ \\
300 \mathrm{~mm} / \mathrm{s}\end{array}$ \\
\hline 15 & 7.98E-06 & 3.38 & 6.76 & 10.14 \\
\hline 16 & 8.67E-06 & 3.67 & 7.34 & 11.02 \\
\hline 17 & 1.02E-05 & 4.34 & 8.68 & 13.02 \\
\hline
\end{tabular}


A regression analysis was employed on the calculated dimensionless parameters using Minitab to generate the empirical model. The Box-Cox transformation was performed on the regression model to ensure accuracy was maintained. A second order polynomial regression was selected based on the highest adjusted $R^{2}(86.73 \%)$ to avoid overfitting. The formed regression equation in terms of three dimensionless parameters is shown in Equation 4-2:

$$
\begin{gathered}
\left(D_{t}\right)^{0.085}=878006 P-431617577200 P^{2}-0.00561 C_{a}+ \\
0.000186 C_{a}{ }^{2}-0.003251 \Lambda+0.00012 \Lambda^{2}-3.094
\end{gathered}
$$

The ANOVA for the transformed response is given in Table 4.10. The "main effects" plots of the mean of fiber diameter as a function of the three dimensionless parameters are presented in Figure 4.19. Also, three surface graphs are also plotted to visualize the effects of the dimensionless parameters. Figure $\mathbf{4 . 2 0}$ demonstrates the trend of fiber diameters in terms of variations in the dimensionless parameters. The surface graphs were produced by plotting two variables on the $X$ and $Y$ axis and the other held at their mean level. The results indicate that both the Processability parameter and Aspect ratio are significant factors $(p=0.000)$. The fiber diameter increased with the increasing Processability parameters, suggesting the combination of low surface tension, high viscosity, and solvent evaporation rate could cause the polymer to resist the thinning process to form a larger fiber. Also, it was discovered that the fiber diameter decreased with the increasing Aspect ratio; the small polymer droplet and long fiber length will prompt the thinning process, forming a smaller fiber. The Capillary number also 
affected the fiber formation with a significance of $p=0.0012$. The Capillary number accounts for the non-instantaneous nature of the direct-write method from the robotic dispensing system. A higher $\mathrm{C}_{\mathrm{a}}$ indicates that drawing is too fast to allow the surface-tension-driven thinning to complete. In contrast, lower $\mathrm{C}_{a}$ illustrates that excessive thinning may occur during the drawing and results in a probable breakup.

Table 4. 10 The ANOVA for the transformed response of the empirical model

\begin{tabular}{lrrrrr} 
Source & DF & Adj SS & Adj MS & F-Value & P-Value \\
\hline Regression & 6 & 4.84915 & 0.808192 & 682.99 & 0.000 \\
Processability parameters & 1 & 0.35384 & 0.353836 & 299.02 & 0.000 \\
Capillary number & 1 & 0.00755 & 0.007551 & 6.38 & 0.012 \\
Aspect ratio & 1 & 0.39703 & 0.397035 & 335.53 & 0.000 \\
Processability parameters*Processability parameters & 1 & 0.28782 & 0.287822 & 243.23 & 0.000 \\
Capillary number*Capillary number & 1 & 0.00241 & 0.002405 & 2.03 & 0.154 \\
Aspect ratio*Aspect ratio & 1 & 0.05332 & 0.053316 & 45.06 & 0.000 \\
Error & 626 & 0.74075 & 0.001183 & & \\
Lack-of-Fit & 345 & 0.59912 & 0.001737 & 3.45 & 0.000 \\
Pure Error & 281 & 0.14164 & 0.000504 & & \\
Total & 632 & 5.58990 & & &
\end{tabular}




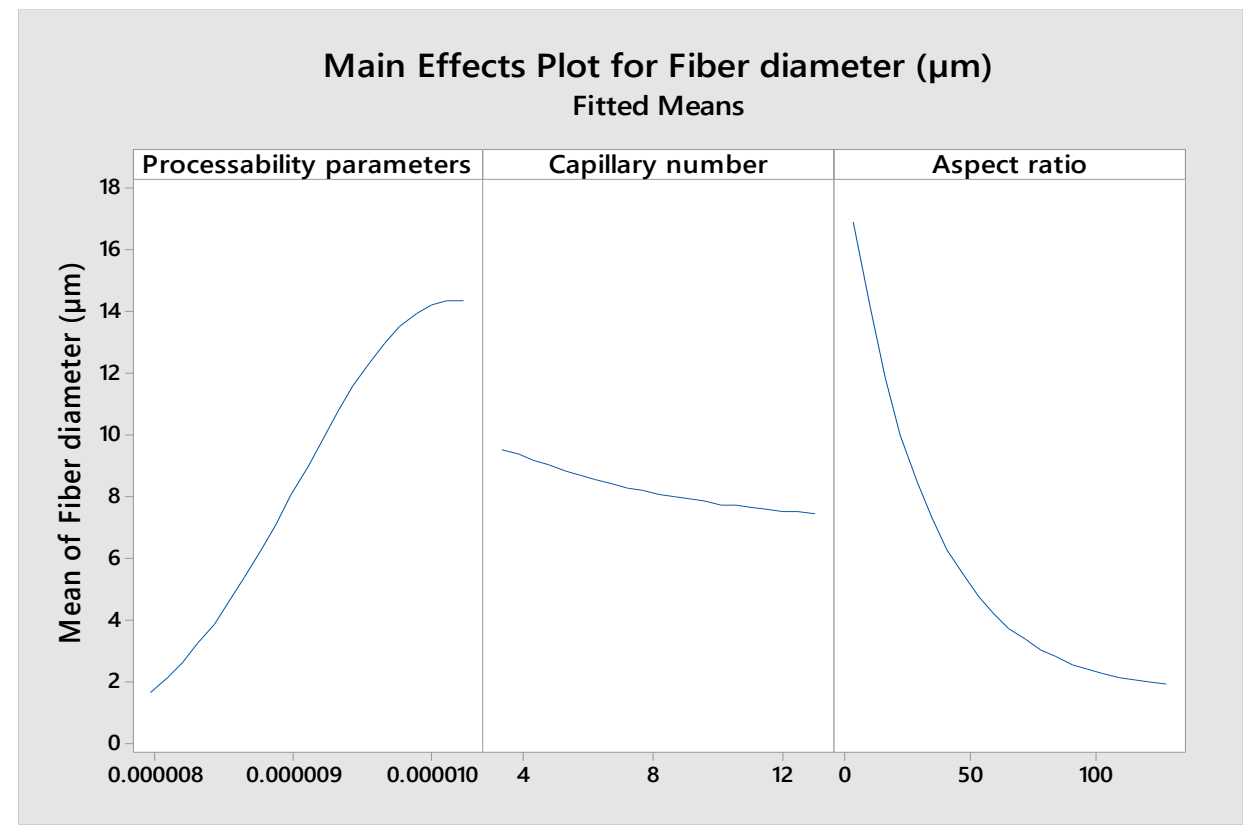

Figure 4. 19 The "main effects" plots of the mean of fiber diameter as a function of Processability parameter, Capillary number, and Aspect ratio. 

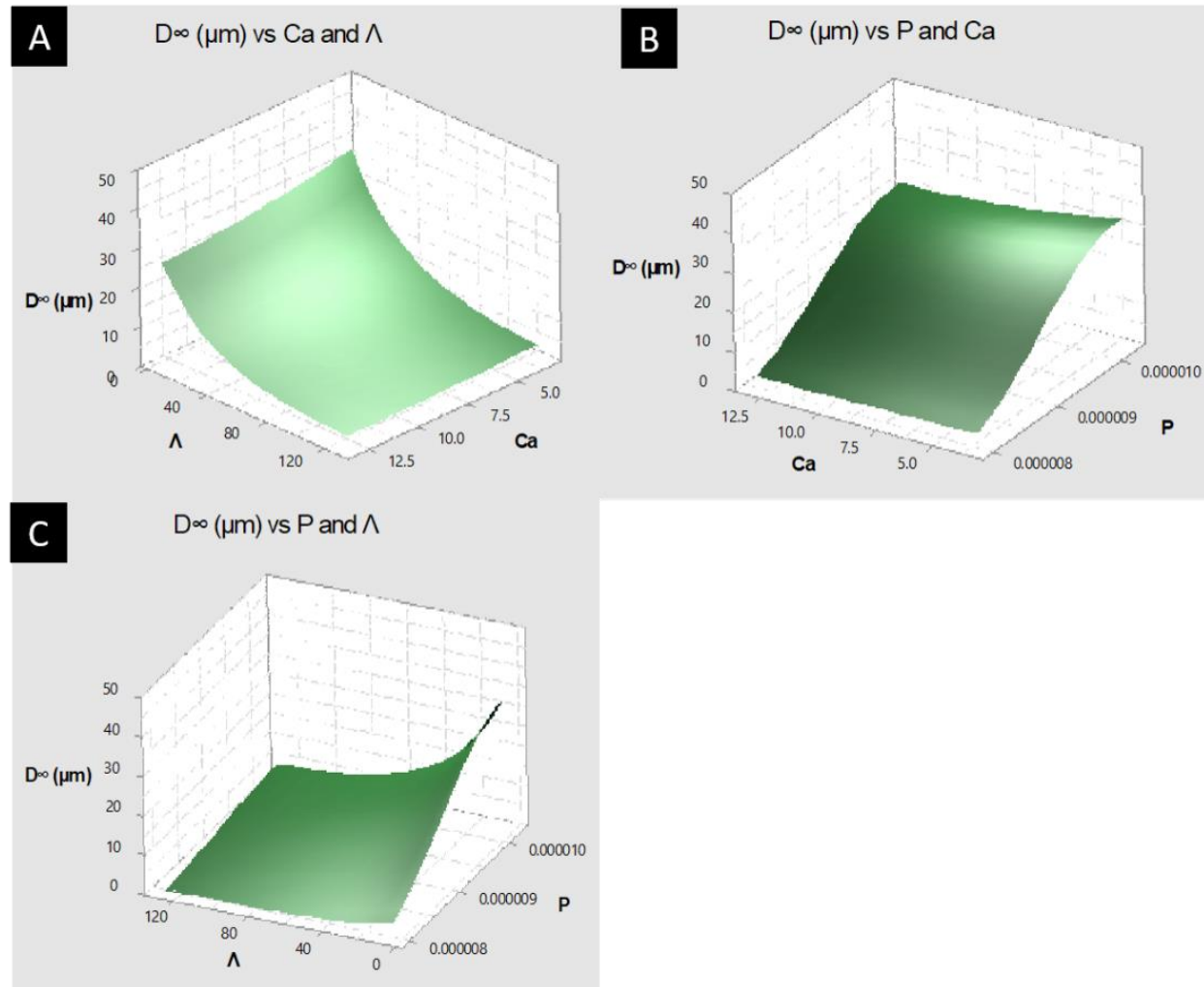

Figure 4. 20 Surface plots representing the fiber diameter as a function of A) Aspect ratio and Capillary number; B) Processability parameters and Capillary number, and C) Aspect ratio and Processability parameters.

The adequacy of the linear regression analysis was checked by residual plots in Figure 4.21. First, the normal probability plot and histogram confirmed that the residuals from the empirical model are approximately randomly distributed. The residuals against fitted values and observation order showed that each residual value is independent from each other. 


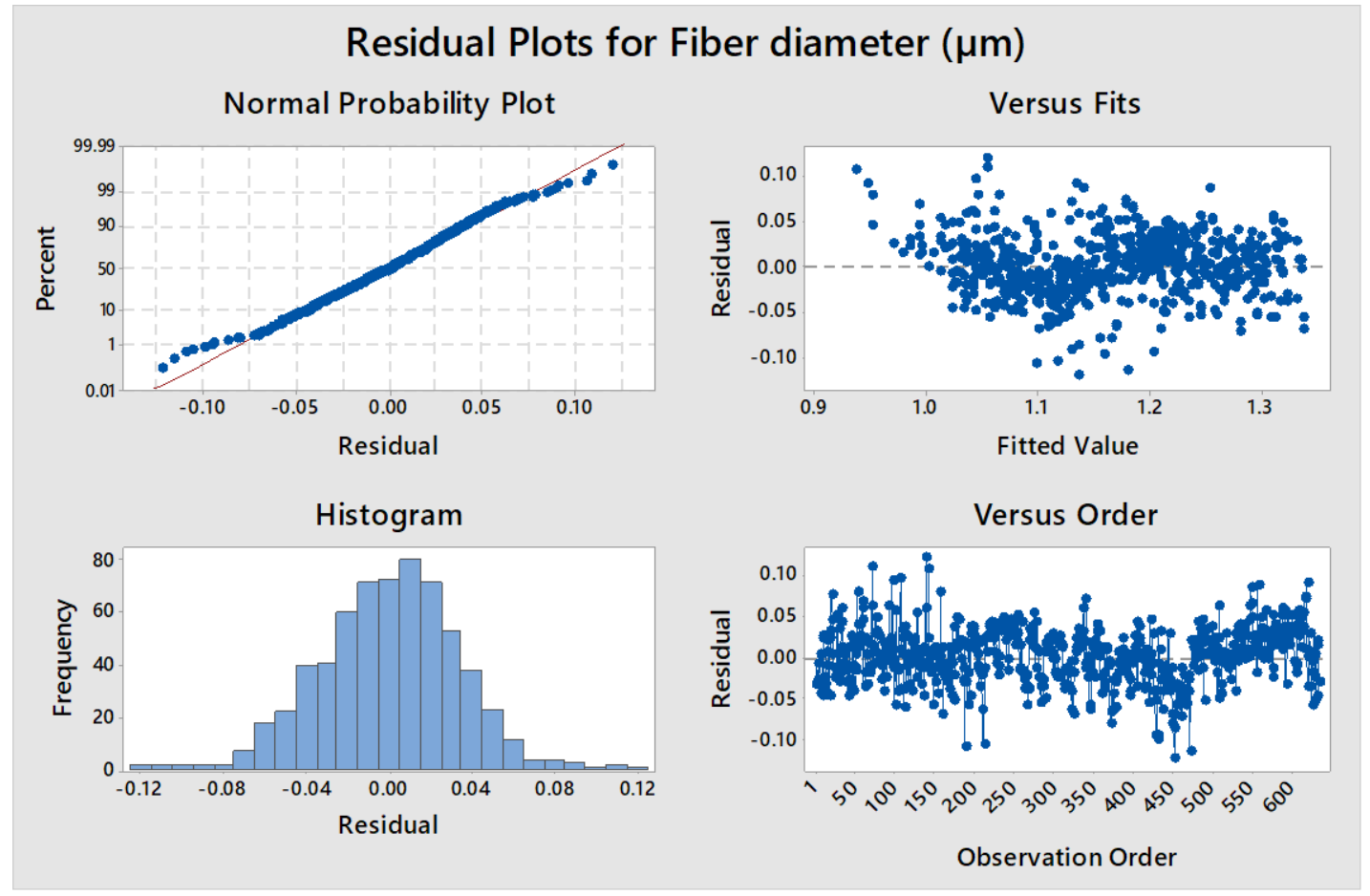

Figure 4. 21 Plots of the normal probability and histogram of residuals, as well as residuals against the fitted value and observation order

\subsubsection{Validation of the Empirical Equation}

The empirical model was generated from the DOE of gelatin solutions in TFE and revealed the correlation between the micro-fiber diameter, the physical properties of the polymer solution, and the direct-write process factors. Since the polymers are a series of long-chain molecules and their solution could be expressed with similar fundamental physical properties, we assume the empirical model could be adopted to predict the diameters of direct-write micro-fibers from other polymer/solvent systems. To test this assumption, we utilized the 3-Axis robotic dispensing system to process various biopolymers into a micro-fiber array. 
The experimental data of the micro-fiber diameters was obtained and compared with the value predicted by the empirical model.

Biopolymers in Table 3.4 were fabricated into a micro-fiber array with fiber lengths of 4,6 , and $8 \mathrm{~mm}$ (three fibers for each length). The yield of those microfibers is shown in Figure 4.22. The yield for most of the produced biopolymer surpassed $65 \%$. However, the yield of $18 w t . \%$ and $19 w t . \%$ gelatin/PLGA (50:50) solution were $32 \%$ and $22 \%$, respectively. The same situation was observed in PLA-PEG (70:30) copolymer fiber; the yield for $30 \mathrm{wt} . \%$ and $31 \mathrm{wt} . \%$ solution were only $11 \%$ and $22 \%$, respectively. We speculate that the combined low viscosity and high surface tension caused the capillary breakup. In addition, the solvent used in the PLA-PEG copolymer is chloroform, which has a lower boiling point than TFE. The solvent would gradually evaporate in the polymer barrel because it is not air-tight. The relatively high volatility may increase the actual concentration of the polymer solution as time passed, leading to the low yield. 


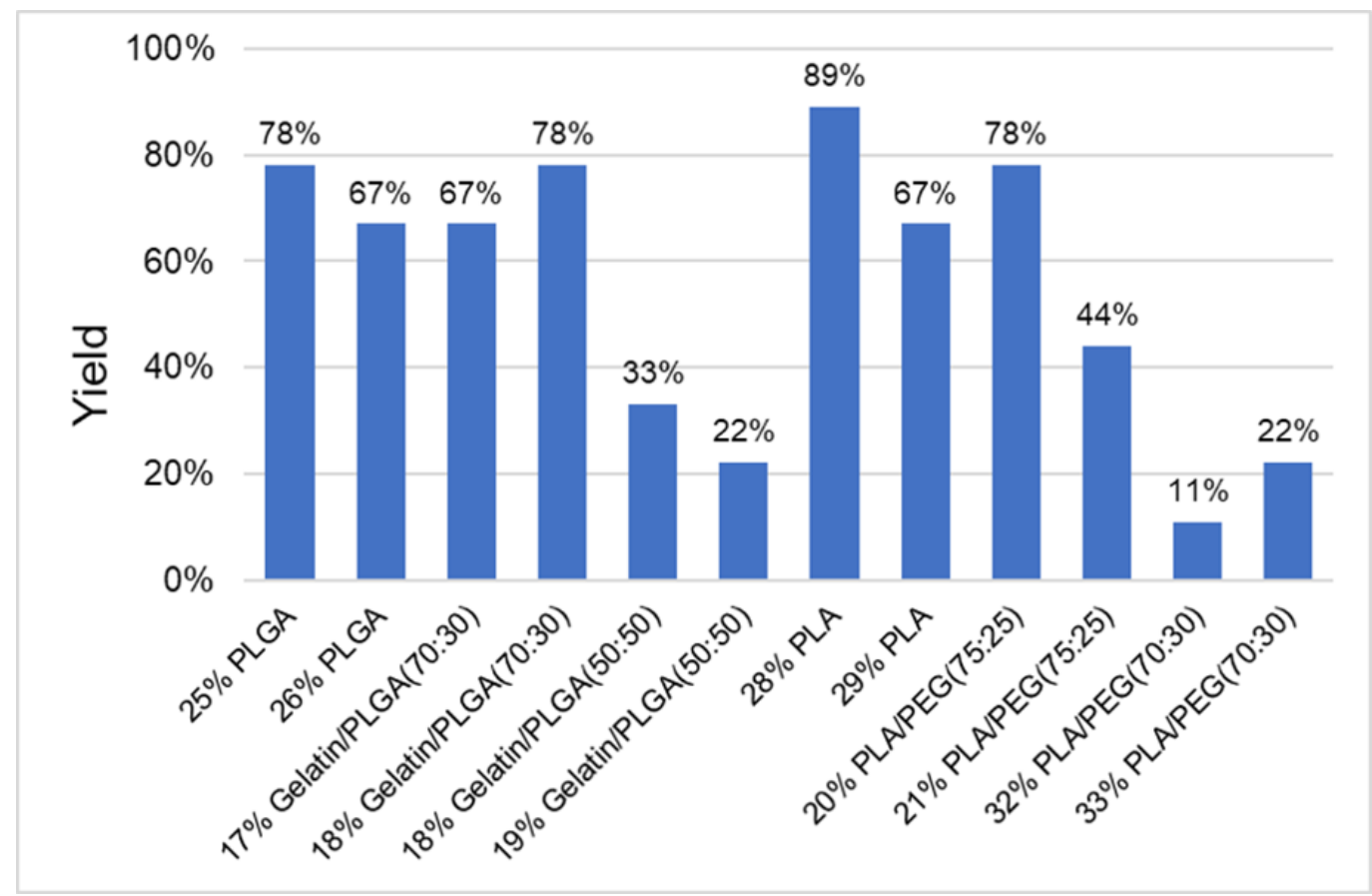

Figure 4. 22 The micro-fiber yield of various direct-written biopolymers

The physical properties of various biopolymer solutions were measured following the previously described method in Chapter 3 . The results are presented in Table 4.11. Next, the dimensionless parameters were calculated based on the obtained physical properties and the process factors (Table 4.12). Then, the calculated dimensionless parameters were used in conjunction with the empirical model (Equation 4.2) to calculate the predicted micro-fiber diameters. Although all the biopolymers solutions could be processed to micro-fibers through the 3-Axis robotic dispensing system, not all the experimental data could be substituted into the model to get the prediction value due to the biopolymers' Processability parameters are excess of exceeding the empirical model range. The distribution of the Processability parameters of the various biopolymers' solution is shown in Figure 4.23. The space between the two orange lines in Figure 4.23 indicates the 
empirical model boundary $\left(\sim 7-13 \times 10^{-6}\right)$, while the Processability parameters of the solutions of $18 \%$ Gelatin/PLGA (70:30) composites, 28\% PLA, 29\% PLA, 32\% PLA-PEG (70:30), and 33\% PLA-PEG (70:30) copolymers are outside of the boundary. Thus, the predictions for those biopolymers are excluded from the empirical model validation.

Table 4. 11 Physical properties of various biopolymers solutions

\begin{tabular}{|c|c|c|c|}
\hline $\begin{array}{l}\text { Concentration } \\
\text { (wt.\%) }\end{array}$ & $\begin{array}{c}\text { Viscosity, n } \\
\text { (Pa*s) }\end{array}$ & $\begin{array}{l}\text { Surface } \\
\text { tension, } \sigma \\
(\mathrm{mN} / \mathrm{m})\end{array}$ & $\begin{array}{c}\text { Mass transfer } \\
\text { coef., X } \\
(\mathrm{m} / \mathrm{s})\end{array}$ \\
\hline $25 \%$ PLGA & $2.12 \pm 0.14$ & $45.55 \pm 1.59$ & $2.40 \mathrm{E}-07$ \\
\hline $26 \%$ PLGA & $2.84 \pm 0.29$ & $65.33 \pm 3.23$ & $2.40 \mathrm{E}-07$ \\
\hline 17\% Gelatin/PLGA(70:30) & $1.02 \pm 0.06$ & $35.17 \pm 2.11$ & $2.54 \mathrm{E}-07$ \\
\hline 18\% Gelatin/PLGA(70:30) & $1.98 \pm 0.11$ & $39.98 \pm 0.92$ & 2.49E-07 \\
\hline 18\% Gelatin/PLGA(50:50) & $0.88 \pm 0.02$ & $56.03 \pm 2.43$ & 2.31E-07 \\
\hline 19\% Gelatin/PLGA(50:50) & $1.38 \pm 0.16$ & $36.03 \pm 2.43$ & $2.26 \mathrm{E}-07$ \\
\hline $28 \%$ PLA & $2.57 \pm 0.08$ & $51.23 \pm 2.66$ & 4.89E-07 \\
\hline 29\% PLA & $3.01 \pm 0.26$ & $49.48 \pm 1.77$ & 4.83E-07 \\
\hline 20\% PLA/PEG(75:25) & $0.98 \pm 0.04$ & $29.88 \pm 0.82$ & $3.14 \mathrm{E}-07$ \\
\hline 21\% PLA/PEG(75:25) & $1.23 \pm 0.15$ & $30.54 \pm 1.24$ & $3.14 \mathrm{E}-07$ \\
\hline $30 \%$ PLA/PEG(70:30) & $0.49 \pm 0.20$ & $58.87 \pm 2.88$ & $3.14 \mathrm{E}-07$ \\
\hline $31 \%$ PLA/PEG(70:30) & $0.66 \pm 0.17$ & $61.41 \pm 3.15$ & $3.14 \mathrm{E}-07$ \\
\hline
\end{tabular}


Table 4. 12 Calculated dimensionless parameters of various biopolymer solutions

\begin{tabular}{lccccc}
\hline \multicolumn{1}{c}{$\begin{array}{c}\text { Concentration } \\
\text { (wt.\%) }\end{array}$} & $\mathbf{P}$ & $\mathbf{C a}$ & $\begin{array}{c}\Lambda \\
(\mathbf{4 m m})\end{array}$ & $\begin{array}{c}\boldsymbol{\Lambda} \\
(6 \mathrm{~mm})\end{array}$ & $\begin{array}{c}\Lambda \\
(\mathbf{8 m m})\end{array}$ \\
\hline 25\% PLGA & $1.12 \mathrm{E}-05$ & 9.31 & 7.63 & 11.45 & 15.27 \\
26\% PLGA & $1.04 \mathrm{E}-05$ & 8.69 & 8.21 & 12.32 & 16.43 \\
17\% Gelatin/PLGA(70:30) & $7.37 \mathrm{E}-06$ & 5.80 & 7.35 & 11.03 & 14.71 \\
18\% Gelatin/PLGA(70:30) & $1.23 \mathrm{E}-05$ & 9.90 & 7.77 & 11.65 & 15.53 \\
18\% Gelatin/PLGA(50:50) & $3.63 \mathrm{E}-06$ & 3.14 & 6.81 & 10.22 & 13.63 \\
19\% Gelatin/PLGA(50:50) & $8.66 \mathrm{E}-06$ & 7.66 & 7.72 & 11.58 & 15.44 \\
28\% PLA & $2.45 \mathrm{E}-05$ & 10.03 & 9.26 & 13.89 & 18.52 \\
29\% PLA & $2.94 \mathrm{E}-05$ & 12.17 & 12.38 & 18.58 & 24.77 \\
20\% PLA/PEG(75:25) & $1.03 \mathrm{E}-05$ & 6.56 & 7.35 & 11.03 & 14.71 \\
\hline 21\% PLA/PEG(75:25) & $1.26 \mathrm{E}-05$ & 8.06 & 7.97 & 11.95 & 15.94 \\
30\% PLA/PEG(70:30) & $2.61 \mathrm{E}-06$ & 1.66 & 6.69 & 10.03 & 13.38 \\
\hline 31\% PLA/PEG(70:30) & $3.37 \mathrm{E}-06$ & 2.15 & 7.21 & 10.81 & 14.41 \\
\hline
\end{tabular}




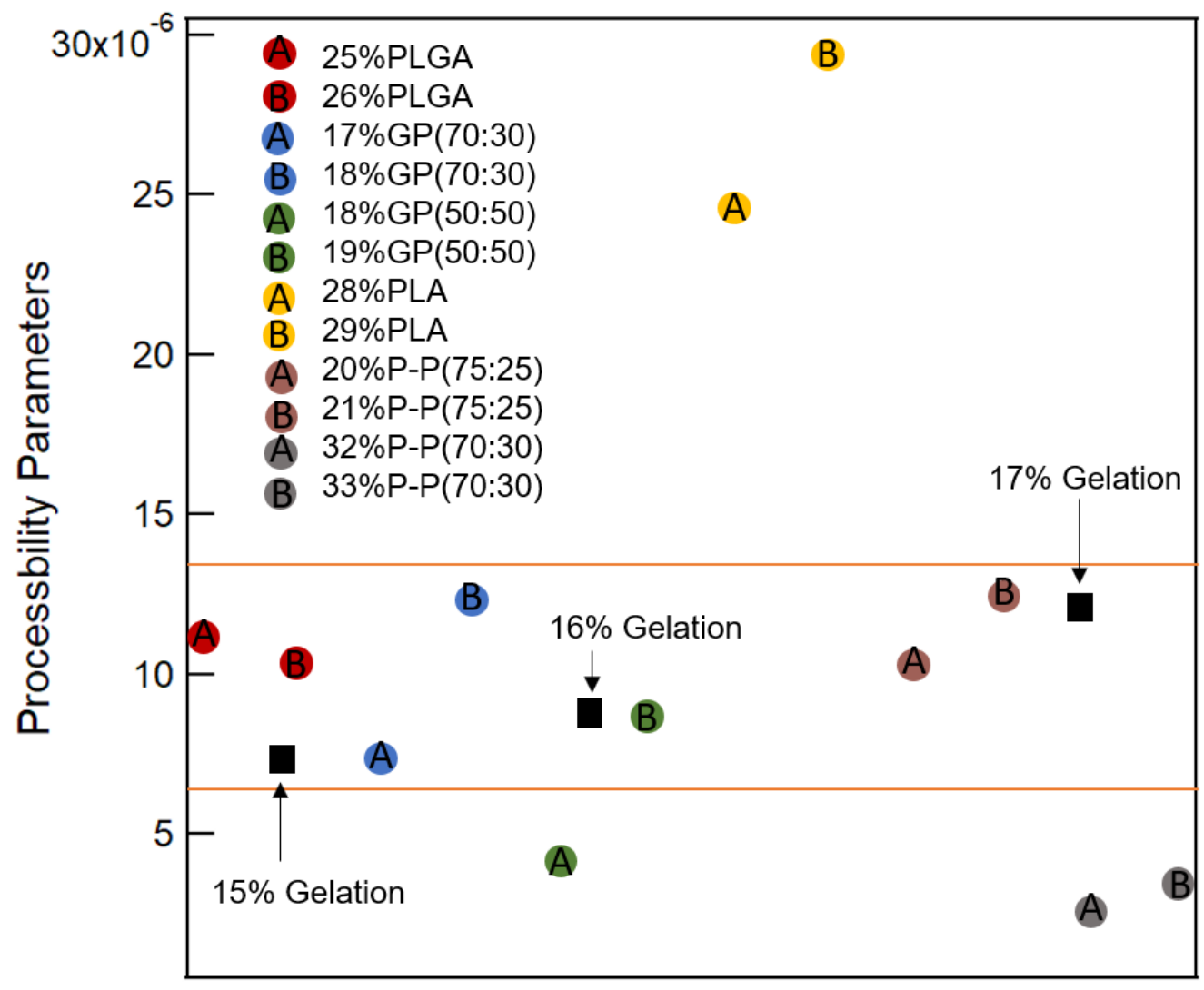

Figure 4. 23 The distribution of the Processability parameters of various biopolymers

The micro-fiber diameters were measured through the SEM as we introduced in section 4.2.1.2. The predicted values were obtained from Minitab by substituting all the calculated dimensionless parameters into the generated model. The experimental and predicted diameters of micro-fibers with lengths of 4,6 , and $8 \mathrm{~mm}$ for the selected biopolymers are presented in Figure 4.24. Generally, the micro-fiber diameter decreased with increasing fiber length. Also, there is a noticeable trend of the micro-fiber diameter increasing with the increasing 
biopolymer solution concentration. The result is consistent with the previously made conclusion that we made earlier in the gelatin fiber characterization process.

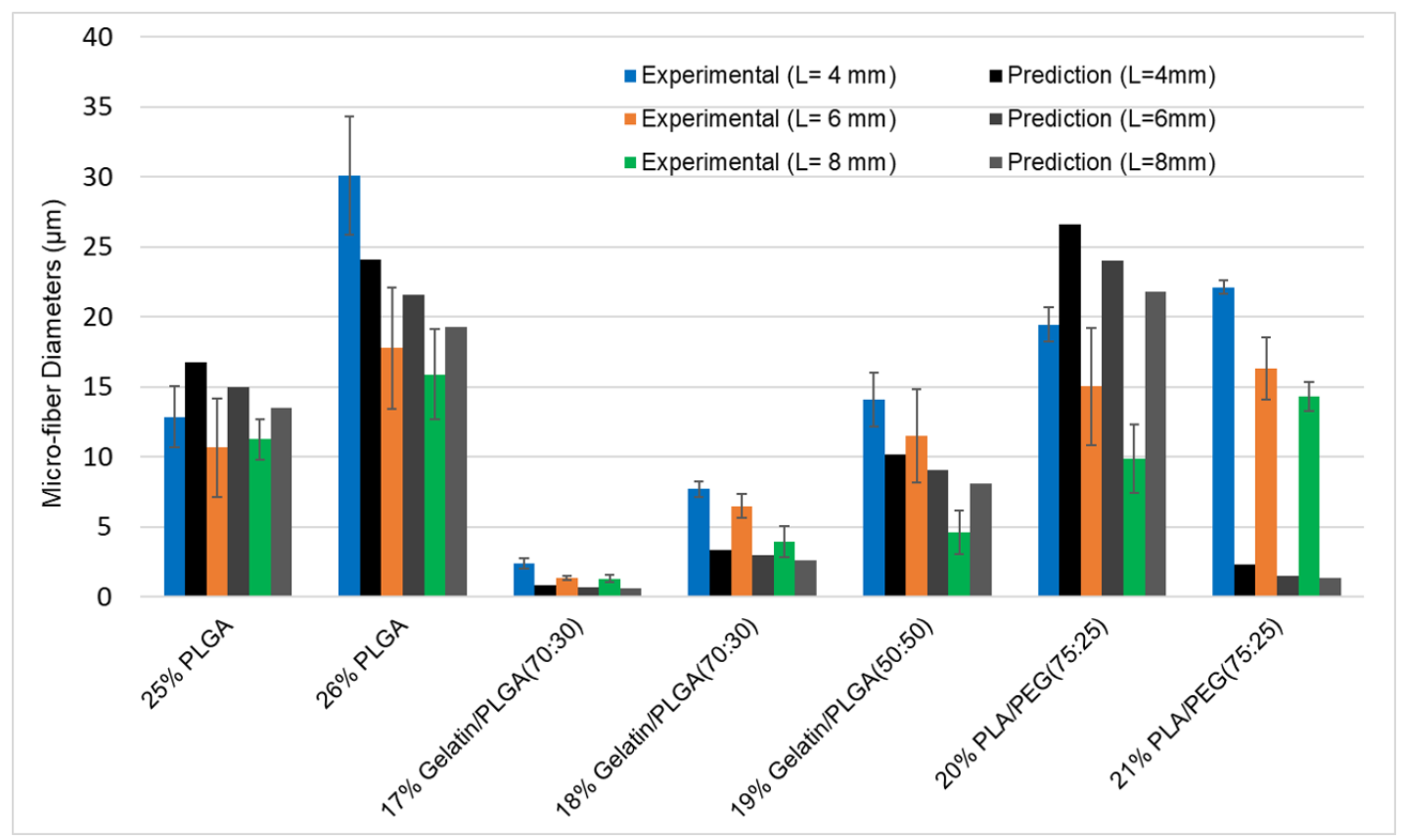

Figure 4. 24 Comparison of the predicted and the experimental diameters of the direct-write micro-fibers for various biopolymers, composites, and copolymers (micro-fiber length of 4,6 , and $8 \mathrm{~mm}$ )

Finally, we used three measures to evaluate the performance of the prediction of the empirical model. The measures are presented in Table 4.13: mean absolute deviation (MAD), mean squared deviation (MSD), and the mean absolute percentage error (MAPE). The mean absolute error and mean absolute percentage error for all validation is $5.77 \mu \mathrm{m}$ and $51.28 \%$, respectively. 
Table 4. 13 Measurement of the prediction error

\begin{tabular}{|c|c|c|c|}
\hline Length & MAD $(\mu \mathrm{m})$ & $\operatorname{MSD}\left(\mu m^{2}\right)$ & MAPE (\%) \\
\hline $4 \mathrm{~mm}$ & 6.65 & 75.70 & 46.61 \\
\hline $6 \mathrm{~mm}$ & 5.51 & 50.34 & 47.98 \\
\hline $8 \mathrm{~mm}$ & 5.15 & 48.80 & 59.25 \\
\hline Average & 5.77 & 58.28 & 51.28 \\
\hline
\end{tabular}

4.3 Direct-write Microvascular Scaffold for Endothelial Cells Morphogenesis

\subsubsection{Biopolymer Selection for Scaffold}

\subsubsection{Biodegradation Test}

From our preliminary cell culture experiments, we found that the gelatin and gelatin/PLGA composites were still water soluble. If made of these polymers, most of the fabricated scaffold will be totally dissolved in the cell medium at $37^{\circ} \mathrm{C}$ within less than 2 hours. Thus, crosslinking of the gelatin and gelatin/PLGA composites was performed to improve the water-resistant ability of the micro-fibers. After comparing common crosslink methods such as oxygen species and glyceraldehyde solutions, the vapor-phase glutaraldehyde method was selected because this is the only method that will not significantly increase the micro-fibers diameters[204]. Therefore, in this experiment, all the gelatin and gelatin/PLGA composites samples were treated with glutaraldehyde vapor (50\%) for 3 hours in a sealed desiccator at room temperature. PLA-PEG (60:40) and PLA-PEG (50:50) 
were not evaluated in this test because they cannot be processed into micro-fibers through the 3-Axis robotic dispensing system.

The samples of the studied biopolymers underwent the biodegradation test in the PBS solution at $37^{\circ} \mathrm{C}$ simulating in vivo conditions. Figure 4.25 shows the biodegradation profiles for various biopolymers. The pure PLA showed the lowest degradation rate, more than $85 \%$ of the weight remained after 8 weeks. However, the PLA-PEG copolymers showed a much higher degradation rate compared to PLA. The PLA-PEG (90:10) and PLA-PEG (75:25) copolymers had $76 \%$ and $47 \%$ of the sample weight remaining, respectively. It should be noted that the PLA-PEG (70:30) broken into small pieces in 4-5 weeks. The reason the copolymer had a higher degradation rate is that the ester bonds formed in the copolymer are broken much easier due to a hydrolytic reaction. It can also be observed that the crosslinked gelatin withstood the aqueous environment more rigorously than traditional gelatin, with $36 \%$ weight loss measured at the end of the experiment. The PLGA degrades slowly, showing a $16 \%$ weight loss. The gelatin/PLGA composites exhibited a moderate degradation rate that was between the rate of the PLGA and gelatin samples. The two composites were observed to break into small pieces after 6 weeks. In summary, although the improvement of the degradation was not ideal, both the composites and copolymers could increase the degradation rate compared to the pure PLA and PLGA. According to this experiment, the crosslinked gelatin, PLA-PEG (75-25), and PLA-PEG(70:30) are all potential scaffold candidates. 


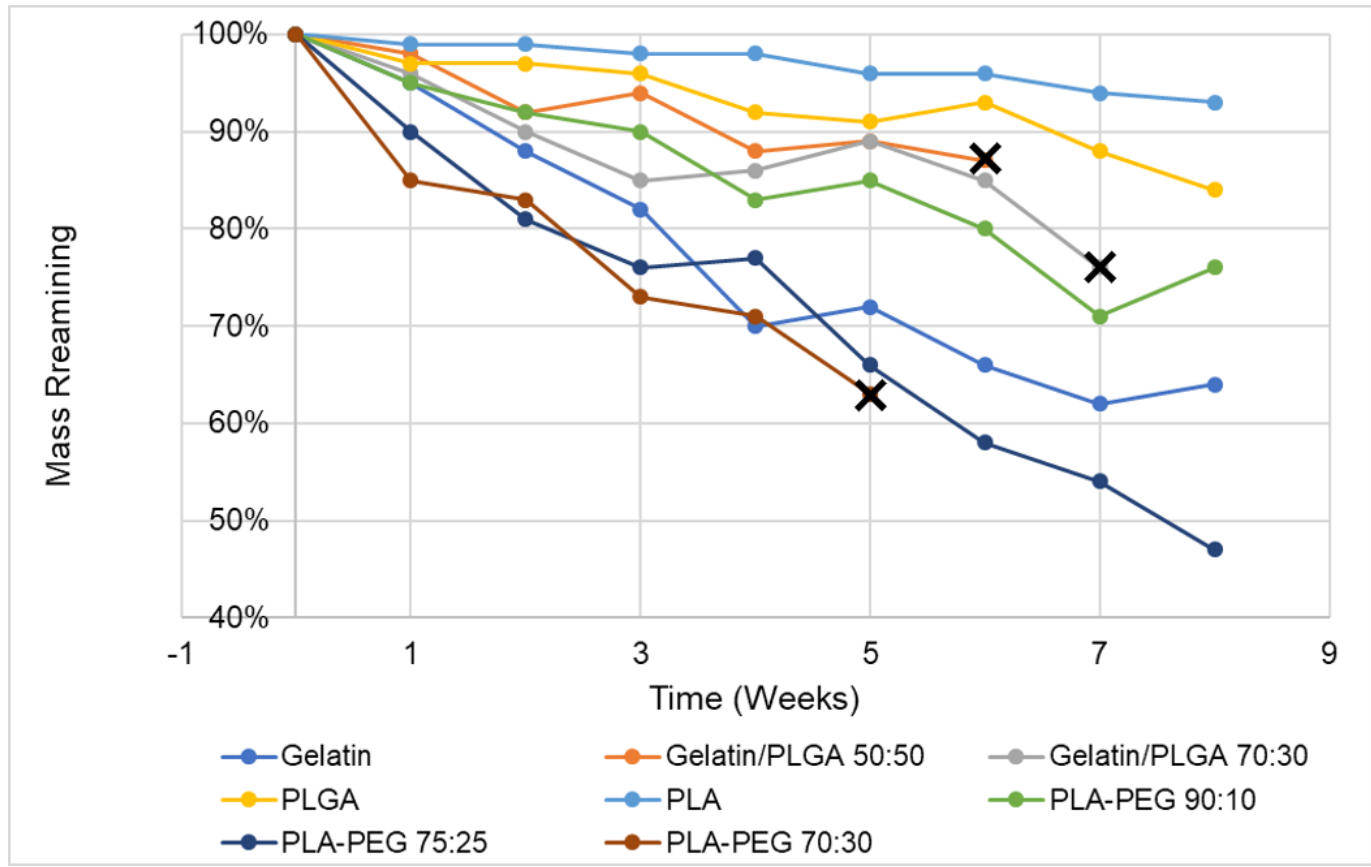

Figure 4. 25 The degradation profile for various biopolymers, composites, and copolymers. Gelatin and gelatin/PLGA were all crosslinked by glutaraldehyde vapor. Error bars were not shown for clear visualization. $\mathrm{X}$ represents that the polymer cubes broke into small pieces.

\subsubsection{Biopolymers Cellular Adhesion and Viability Test}

After evaluating the biopolymer's degradation ability, it is important to examine if the biopolymers are suited for the HDMECs growth. The cellular adhesion potential of the biopolymers and the viability test results are presented in Figure 4.26. The green and red color represent the live cells and dead cells, respectively. It could be seen that only a few viable cells were detected in the crosslinked gelatin and gelatin/PLGA composites. One possible reason for this is the toxicity of the crosslinking agent - glutaraldehyde. It has been reported that the potential source of cytotoxicity of the crosslinker may be residue of unreacted 
crosslinking agent that leaches into the cell media. We also initially speculated that the cytotoxicity may be a result of the solvent TFE. However, this speculation could be ruled out because the PLGA dissolved in TFE (not treated by the glutaraldehyde vapor) possesses a much higher viable cell count. The PLA and PLA-PEG copolymers generally showed high cell viability. Although the PLA-PEG copolymers with improved hydrophilicity were expected to grow more cells than PLA, we observed that the attached cell count for the copolymers was less than the PLA; the attached cell count decreased with increasing the content of PEG segments in the copolymer. This trend has also been reported before: PEG segments could reduce the binding of proteins and cell adhesion on the material surface[205]. Thus, PLGA, PLA, and PLA-PEG copolymers are all potential biopolymers for the HDMECs seeding and growth.

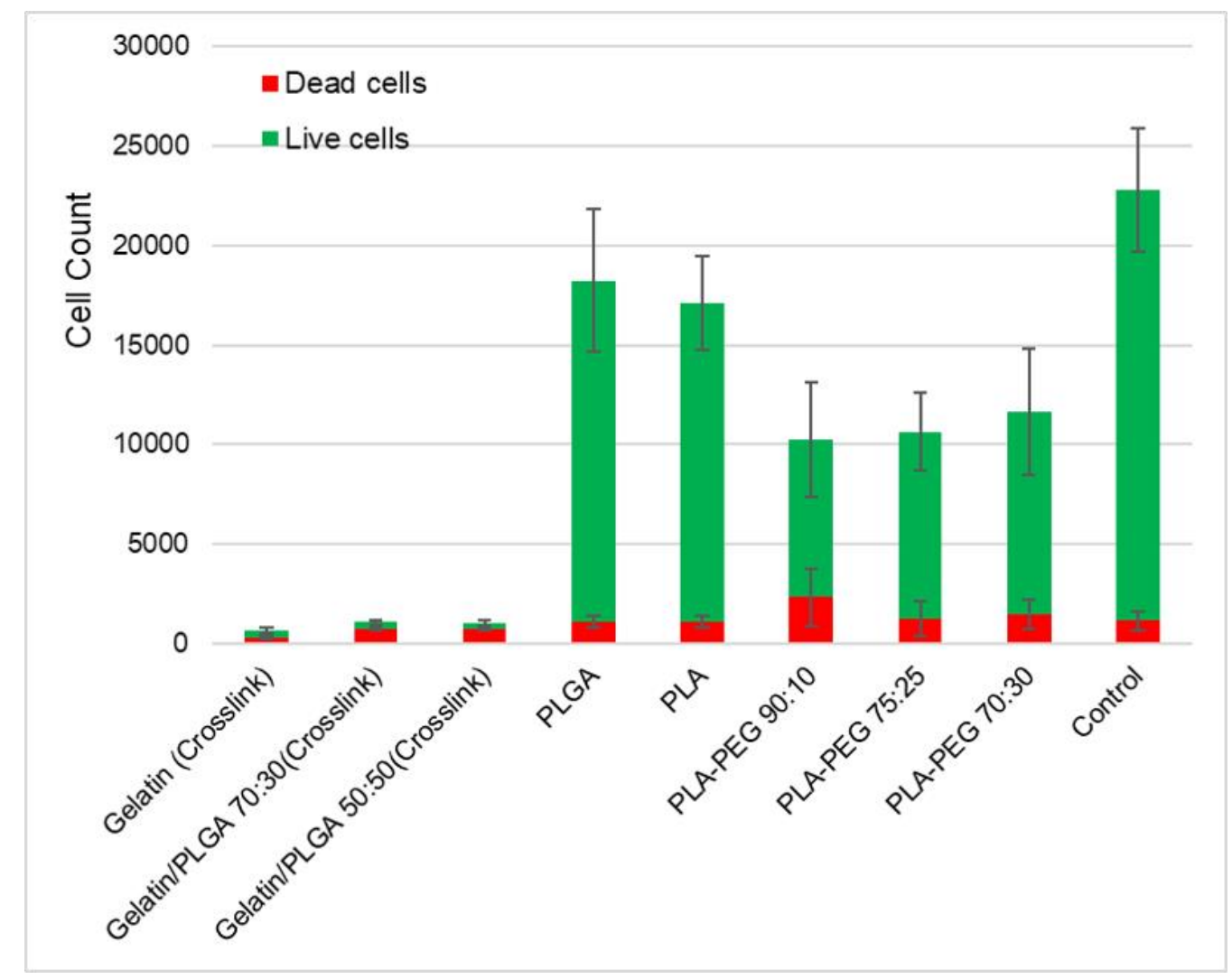


Figure 4. 26 Cellular adhesion and viability profiles for various biopolymers

Considering the multiple factors involved in the direct-write yield, the biodegradation rate, and the HDMECs adhesion ability and viability comprehensively, the PLA-PEG (75:25) copolymer was selected for the microvascular scaffold study.

\subsubsection{Cell Seeding Conditions}

\subsubsection{BSA Coating Experiment}

A critical goal of this project is to ensure the HDMECs could adhere to the suspended scaffold and proliferate on it. Besides the gentle rocking of the well plate to increase the possibility that the suspending cells could contact the scaffold, we could also block the bottom of the well plate. The BSA coating has proved to be one of the efficient ways to avoid cell adhesion on the bottom on the well plate for HUVECs[201]. Figure 4.27 shows the blocking effects of different concentrations of the BSA coating for HDMEC cell adhesion. It can be concluded that the BSA coating significantly reduced the cells number that attached on the bottom of the well plate $(p<0.002)$. The result also indicated that the concentration of BSA did not influence the blocking of the HDMECs, possible because that even the BSA solution with concentration as low as $0.01 \%$ was enough to form a uniform anti-fouling layer on the bottom of the 24-well plate and prevent the HDMECs adhesion. 


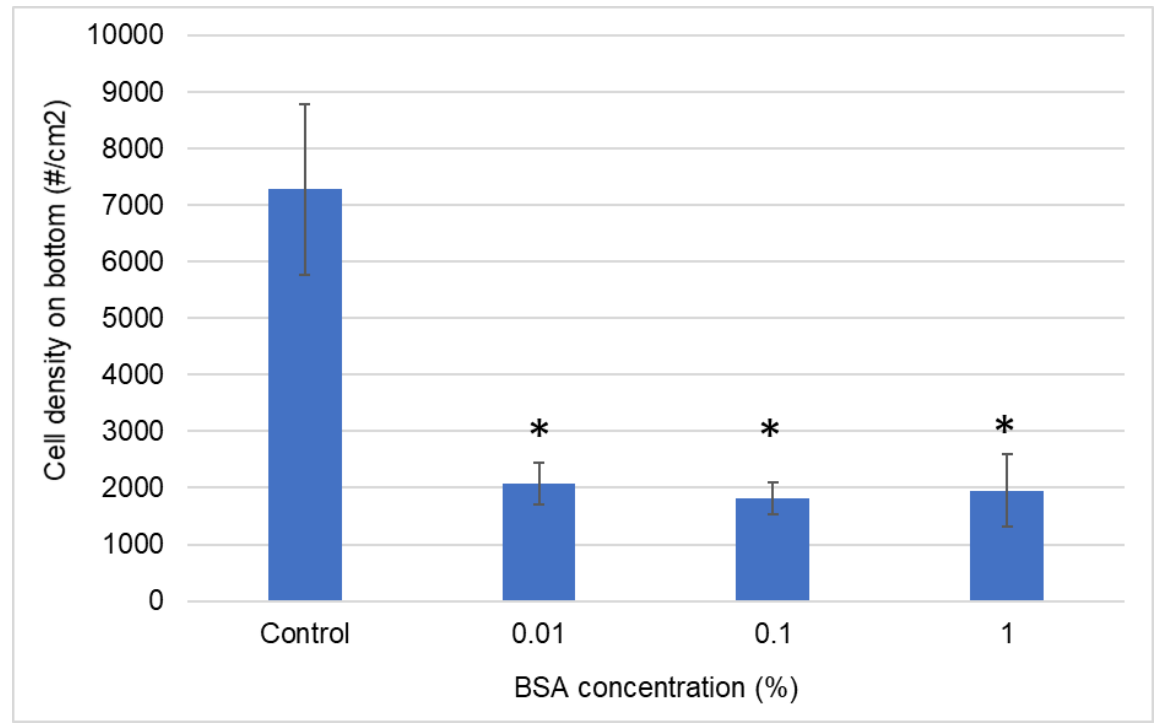

Figure 4. 27 The comparison of cell density on the bottom of the well plate after applying a BSA coating at different concentrations

\subsubsection{Adhesion Promoters Comparison}

In order to further enhance the adhesion of the HDMECs on the surface of the scaffold, adhesion promoters such as gelatin-based attachment factor (AF) and fibronectin (FN) were applied on both PLA-PEG (75:25) copolymer films and microfibers. Figure 4.28 displays the fluorescent images (nuclei with DAPI) of the HDMECs adhered on the copolymer films and micro-fibers with different adhesion promoters. The statistical result is presented in Figure 4.29. With the surface modifications of $\mathrm{AF}, \mathrm{FN}$, and their combination, the attached cells number on the films had a higher cell count than the non-treatment control $(p=0.101, p=0.030$, and $p=0.044$, respectively). It can be observed that the difference in successful adhesion between the FN and AF+FN treatments is not significant $(p=0.738)$. 


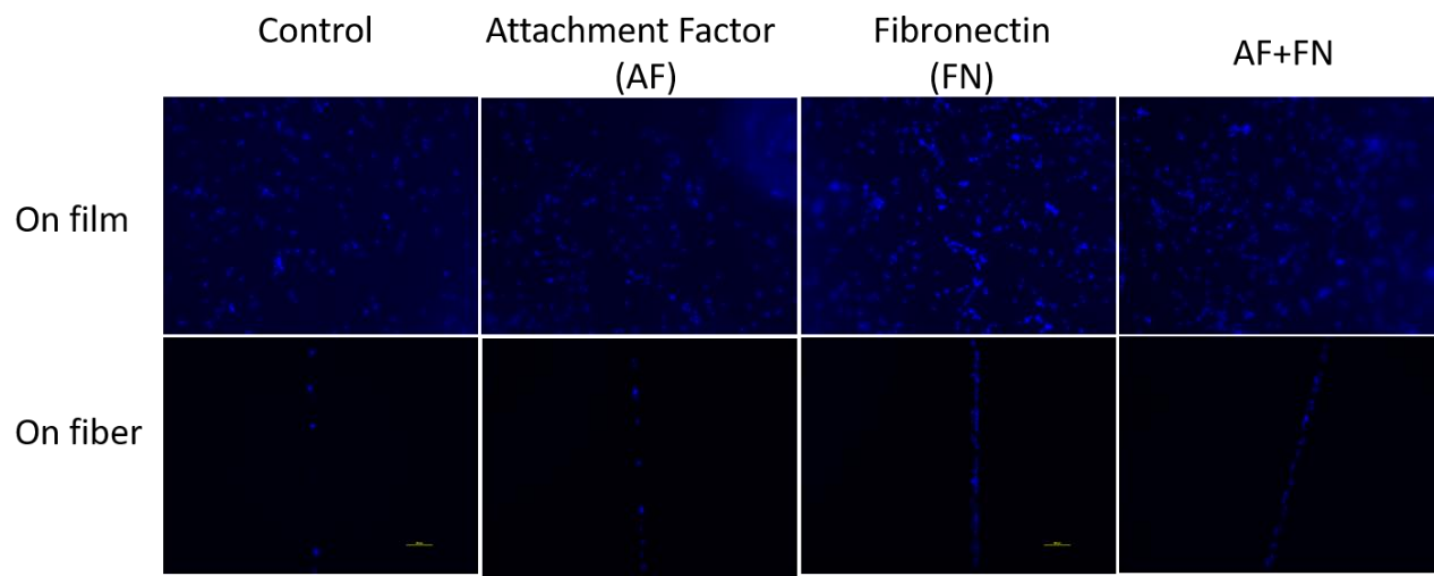

Figure 4. 28 Fluorescent images of the HDMECs adhered on copolymer films and micro-fibers with different adhesion promoters

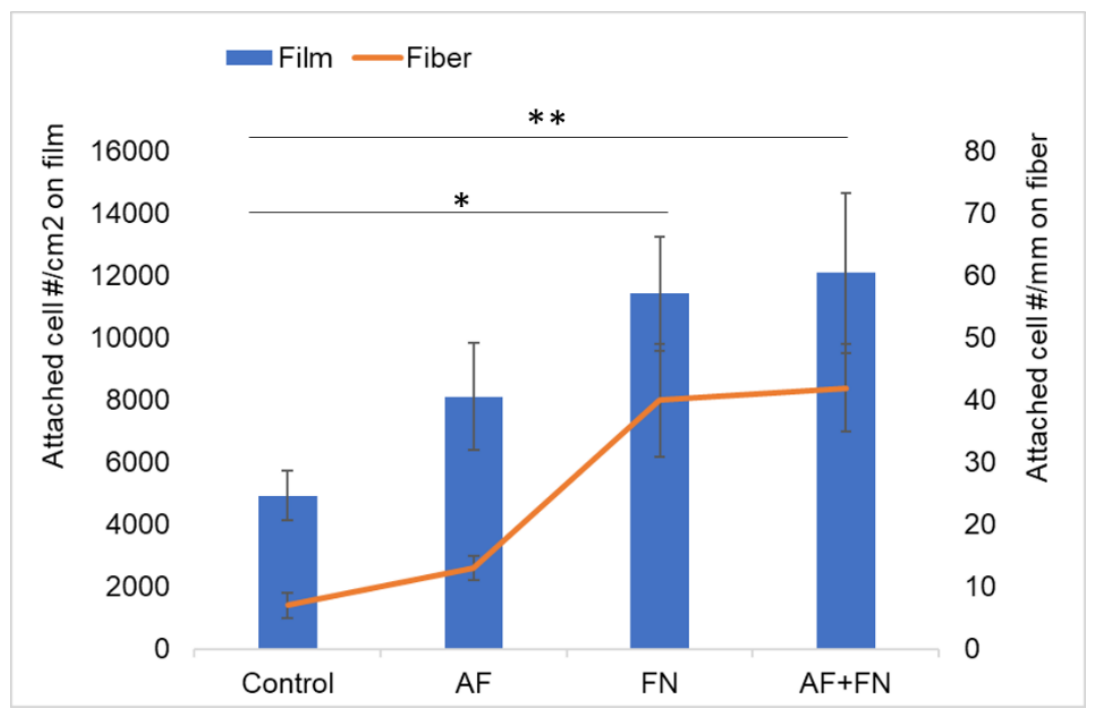

Figure 4. 29 The quantity of attached cells on the copolymer films and micro-fibers with treatments of different adhesion promoters 


\subsubsection{Cell Seeding Density Experiment}

The preliminary culture experiments indicated that the initial HDMECs seeding density of approximately 40,000 cells $/ \mathrm{cm}^{2}$ was insufficient due to most of the scaffold only having cells scattered on its surface. According to our seeding strategy, the suspended cells would randomly adhere and grow on the scaffold under the influence of gravity and external stimulation, such as rocking. We anticipate the increasing cell seeding density could improve the output. Figure 4.30A-D shows the fluorescent images of the HDMECs adhered on the copolymer micro-fibers with a series seeding density: 40,000 cells $/ \mathrm{cm}^{2}, 60,000$ cells $/ \mathrm{cm}^{2}$. 80,000 cells $/ \mathrm{cm}^{2}$, and 100,000 cells $/ \mathrm{cm}^{2}$. Figure 4.30E illustrates the statistical results of this experiment. It can be seen that when increasing seeding density, the number of adhered cells on the length of the micro-fiber increased significantly ( $p$ $=0.013, p=0.001$ and $p=0.000$, respectively). Although a higher initial seeding density could obtain a higher cell count on the micro-fiber, the result suggests that the difference between 80,000 and 100,000 cells $/ \mathrm{cm}^{2}$ is not significant $(p=0.800)$. Considering the cost and time involved in culturing cells, we chose the seeding density of 80,000 cells $/ \mathrm{cm}^{2}$ for the future experiment. 

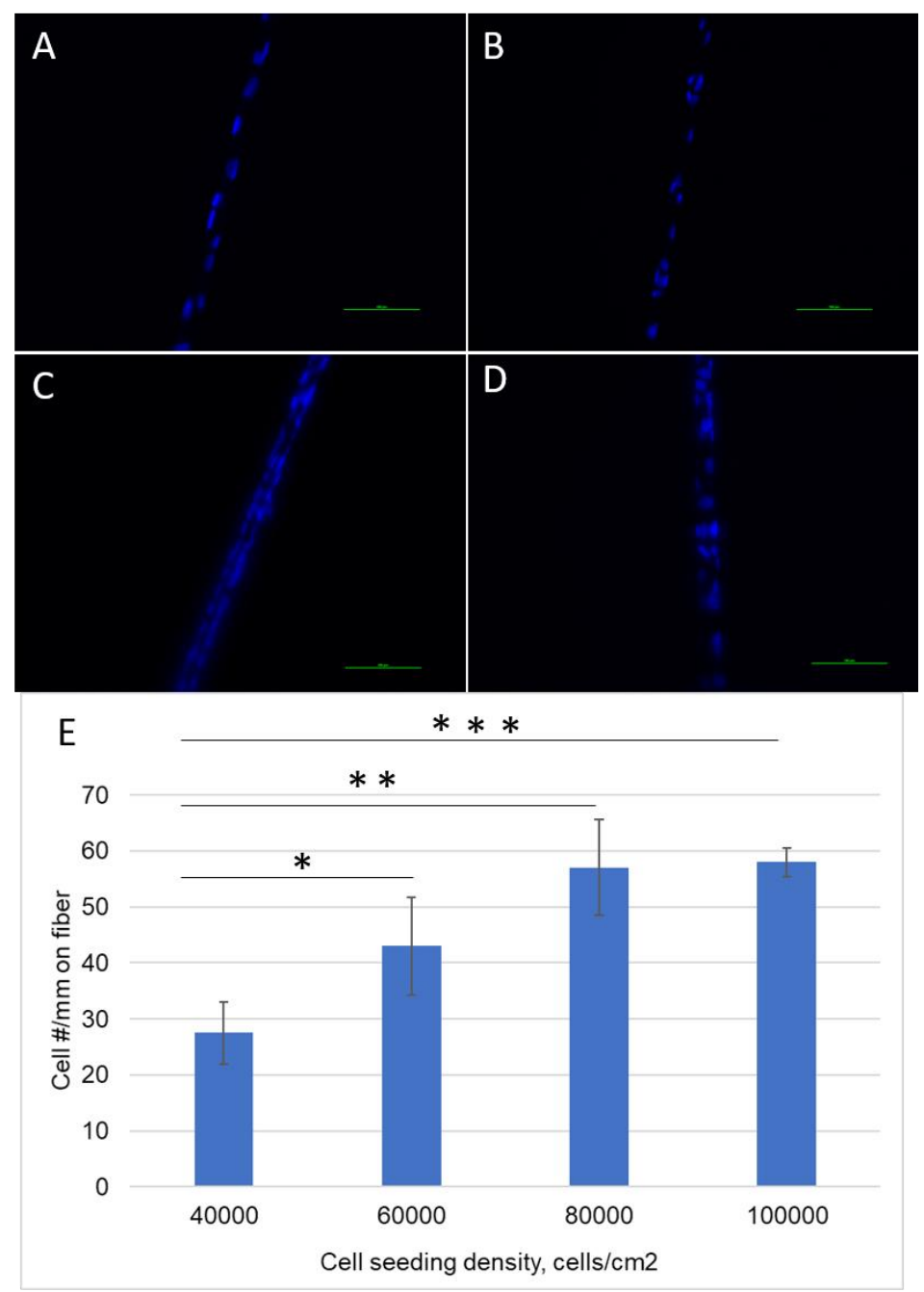

Figure 4. 30 Fluorescent images of the HDMECs adhered on the micro-fiber with seeding density of A) 40000 cells/cm2, B) 60,000 cells/cm2, C) 80,000 cells/cm2, and D) 100,000 cells/cm2. E) Differences in the quantity of the attached cells on the micro-fibers with a different seeding density.

\subsubsection{Endothelial Cell Tubulogenesis on Microvascular Scaffold}

With the previously obtained optimal seeding conditions, the HDMECs were successfully seeded on the scaffold and cultured for 48 hours without any addition 
of exogenous growth factors or angiogenic agents. The slice view of the confocal image of a single PLA-PEG micro-fiber with HDMECs cultured and stained is presented in Figure 4.31A. As can be seen in this slice view (X-Y view), the cells preferentially spread along the axial direction of the micro-fiber scaffold. The scaffold displayed in the figure had a diameter of $12.4 \mu \mathrm{m}$, and the average distance between adjacent cells was $15.1 \pm 3.2 \mu \mathrm{m}$. The circular shape in the right of Figure 4.31A is the $X-Z$ view; this image demonstrates the two cells connected circumferentially around the scaffold and formed a lumen. Figure 4.31B shows the confocal volume view of the HDMECs covered scaffold. From the 3D view, it can be observed that the whole surface of the scaffold was covered with the cells along the axial direction. The inserted cross-sectional image confirmed the confluent monolayer formation of the HDMECs due to the cells wrapping circumferentially around the scaffold. In Figure 4.31C-D, the HDMECs demonstrated an elongated shape on the surface of the three-dimensional branched micro-fiber with an aligned orientation that was different from the typical two-dimensional spread shape with random orientation. The morphological change of the cells suggests a distinctive cellular response to the three-dimensional scaffold. The result also confirmed the previously reported contact guidance phenomenon whereby the endothelial cells follow the direction lead of natural or artificial scaffolds architecture to mediate their morphogenesis and functional differentiation[195]. 


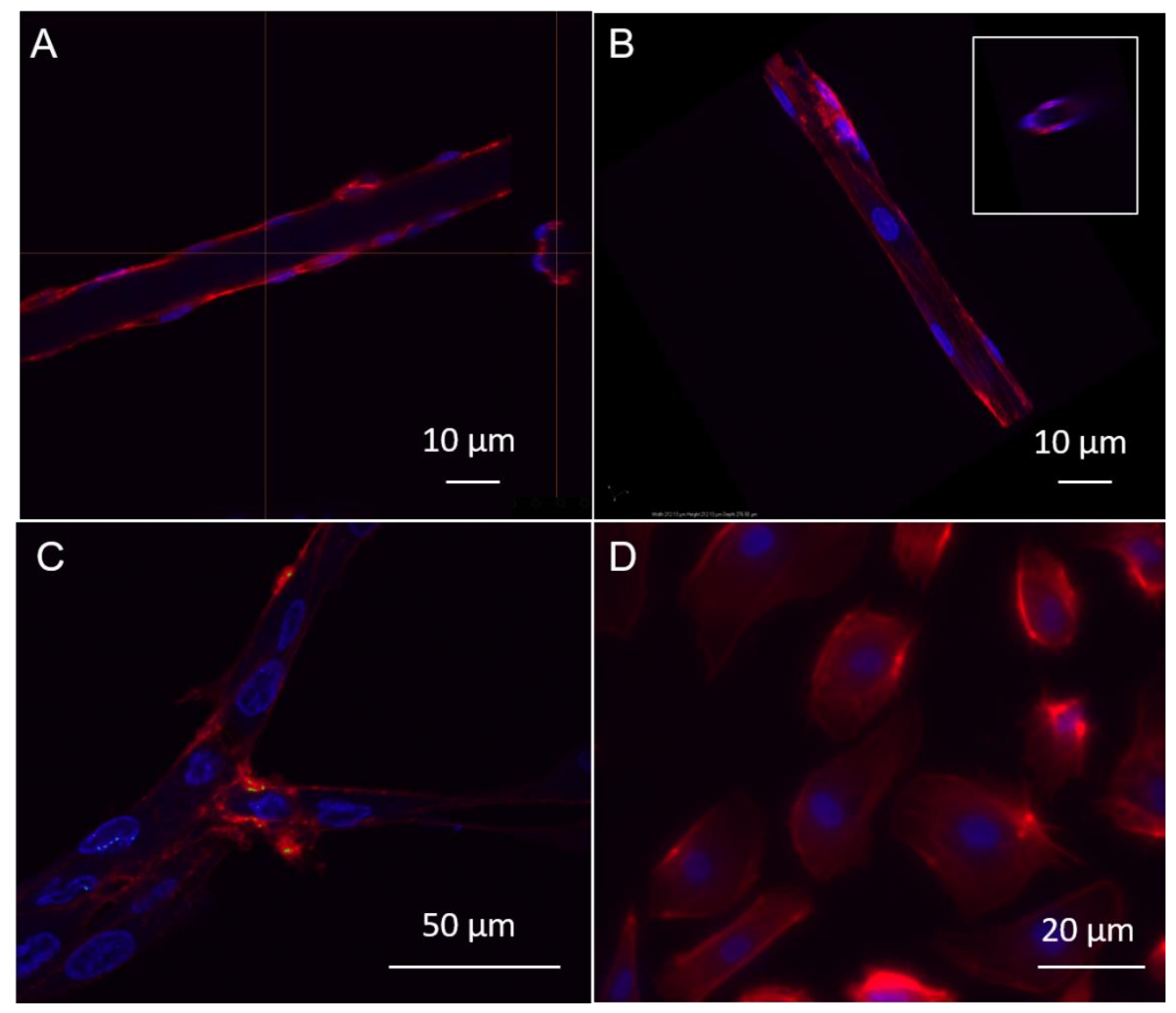

Figure 4. 31 Confocal images of a single PLA-PEG fiber with the HDMECs cultured and stained to highlight the nuclei (blue) and cytoskeletons (red) A) Slice view; B) Volume View; Fluorescent images of the HDMECs stained to highlight the nuclei (blue) and actin cytoskeletons (red) at C) the surface of a branched PLA-PEG scaffold, and D) the bottom of the 24 well plate

In order to further study the physiological characteristics of the HDMECs growing on the scaffold, immunostaining for vascular endothelial cadherin (VEcadherin) was employed to identify cell-cell junctions. Figure 4.32A presents a single PLA-PEG copolymer scaffold with its surface covered by the HDMEC 
monolayer. The inserted cross-sectional image confirmed the cells have grown around the circumference of the scaffold. In Figure 4.32B, the cell-cell junctions were demonstrated via VE-cadherin expression. Recent findings have uncovered that endothelial cell-cell junctions play an essential role in maintaining structural integrity and transferring intracellular signals that determine cell growth, cell polarity, lumen formation, and interaction with pericytes and smooth muscle cells[206]. Additionally, the endothelial cell-cell junctions have been involved in several complex signal transmission that controls the vascular permeability and barrier function in an adult vessel[207]. The VE-cadherin expression of the HDMECs on the scaffold demonstrated the neighboring cells adhered to each other and formed cell-cell junctions. These images also suggest that the cellcovered scaffold strategy has the ability to form a microvascular network in vitro.

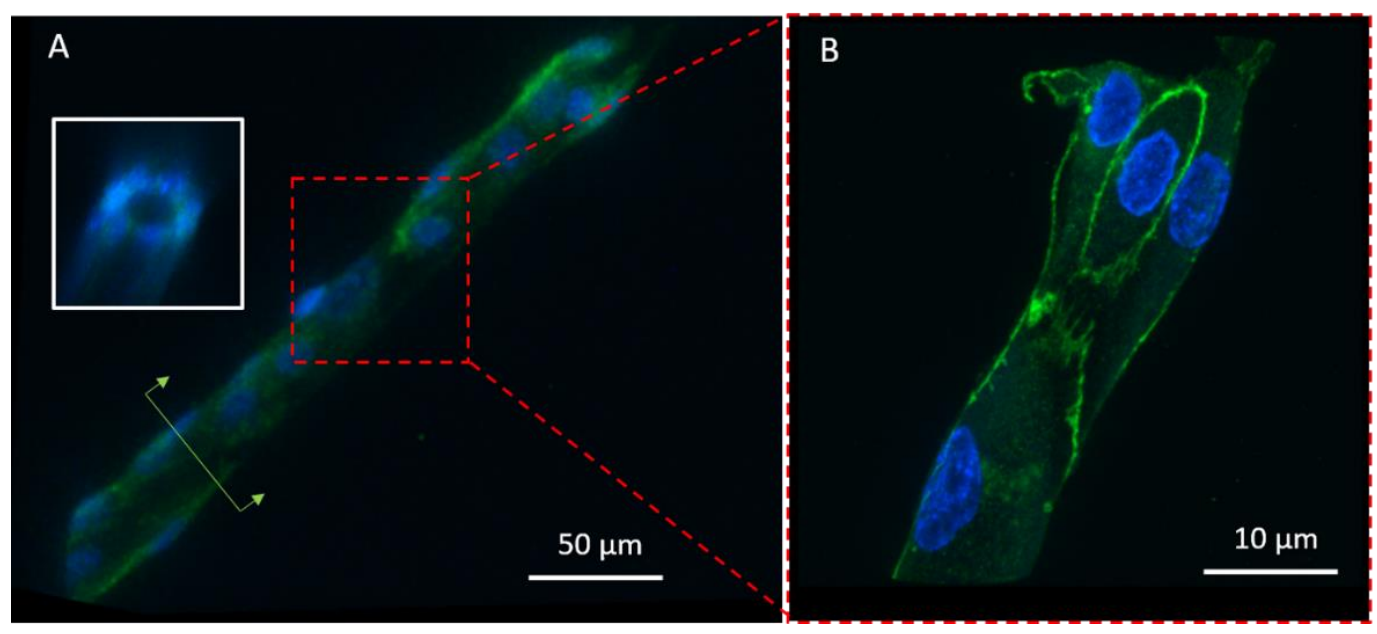

Figure 4. 32 Confocal image of a single PLA-PEG fiber with the HDMECs cultured and stained to highlight the nuclei (blue) and VE-cadherin (green) with a crosssectional image (insert) at 40X magnification $A$ ), and $B$ ) partial enlargement of $(A)$ at $60 \mathrm{X}$ magnification. 
The HDMECs grown on the PLA-PEG copolymer were imaged at higher resolution by using the SEM. As illustrated in Figure 4.33A, similar results for the previous immunostaining confocal images were observed in the SEM images. The scaffold displayed in the figure had a diameter of $13.1 \mu \mathrm{m}$, and the average size of the HDMECs was $17.6 \pm 2.86 \mu \mathrm{m}$. After the fixation and dehydration, the HDMECs were observed to be evenly distributed on the surface of the scaffold. It can also be observed that the long axial of the elongated cells aligned along the axial direction of the scaffold. Figure 4.33B-D showed several enlarged images of connections between adjacent cells (pointed with the red arrow); these images could further confirm the formation of the cell-cell junctions. 


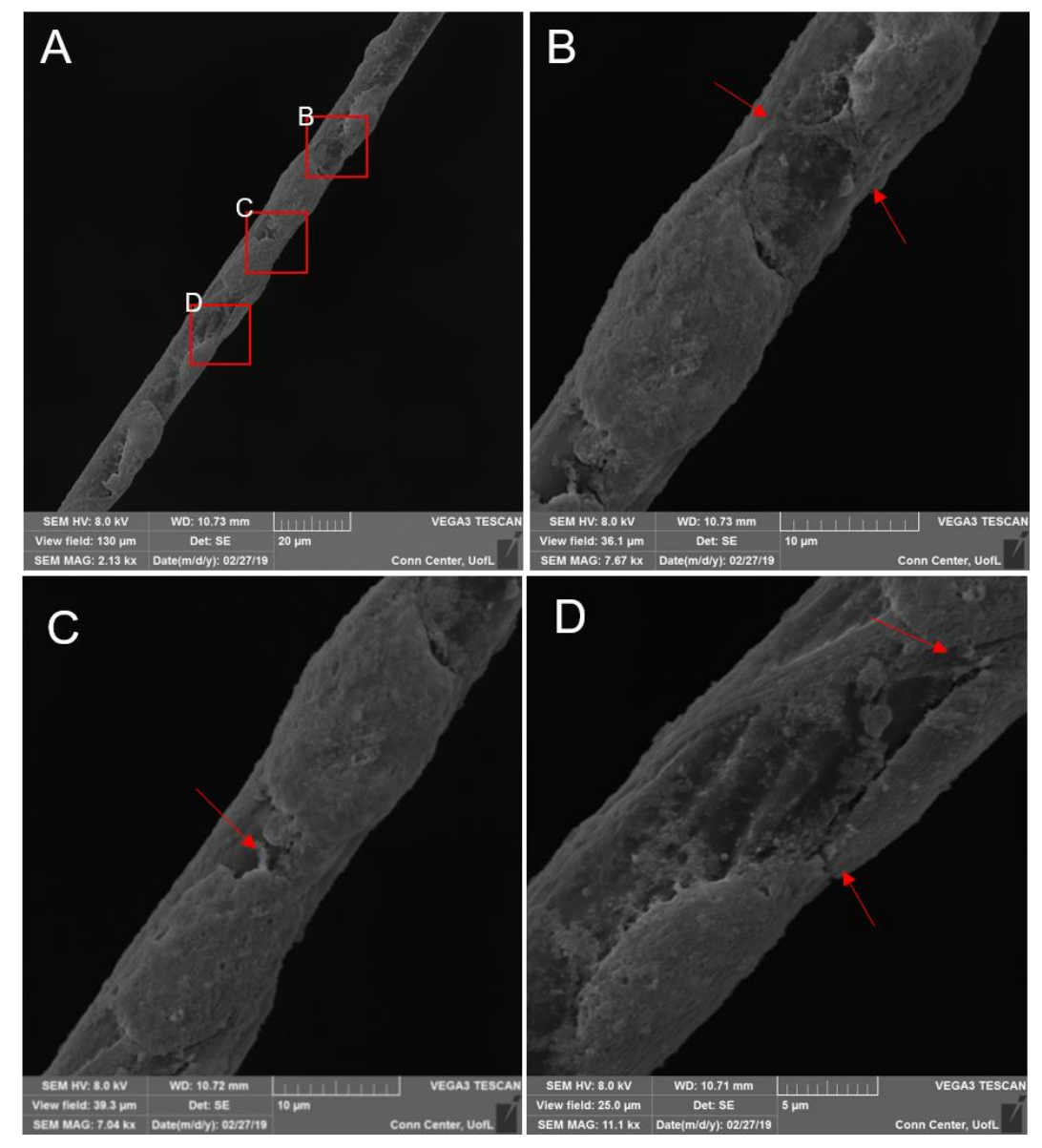

Figure 4. 33 A) SEM image of a single PLA-PEG fiber with the HDMECs cultured for 48 hours; B), C), and D) partial enlargements of (A) for cell-cell junctions

In order to determine whether the cells on the surface of the PLA-PEG scaffold are still alive after two days of culturing, a live/dead assay was utilized to examine the viability of the HDMECs on the scaffold. Figure 4.34 presents the fluorescent images with different magnifications: A) 4X, B) 10X, and C) 20X. The upper row images were captured under the blue filter showing the live cells, and the lower row images were taken under the green filter showing the dead cells. It can be seen that the cells on the suspended scaffold are all alive and grew along 
the axial direction of the scaffold. Several dead cells could be observed in the background, which is the bottom of the 24 -well plate at a different focal plane. This result suggests the cells that cover the scaffold are still active and could be cultured in the ECM for the further study.

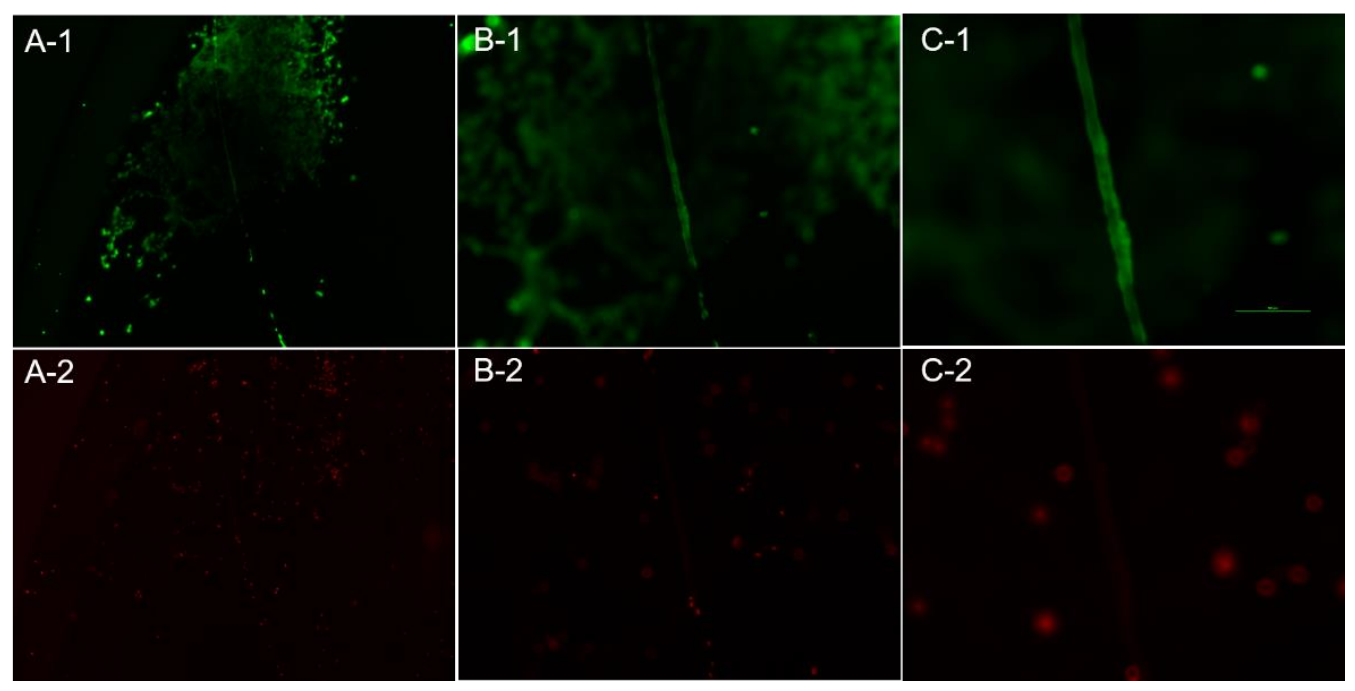

Figure 4. 34 Fluorescent images of the HDMECs stained to highlight the live cells (Calcein, green, upper row) and dead cells (EthD-1, red, lower row) at different magnification: A) 4X, B) 10X, and C) 20X

After demonstrating the ability to grow a confluent HDMEC monolayer on the single PLA-PEG micro-fiber scaffold, the HDMECs were also seeded and cultured for 48 hours on a PLA-PEG branched structure. The 3D reconstruction confocal image is presented in Figure 4.35A. It can be seen that the cells were patterned along the branched structure with topographic guidance. No cell connections between the neighboring branches were observed, which further confirmed the cellular orientation response to the three-dimensional scaffold. 
Figure 4.35B shows the partial enlargement of the branched structure; similar results compared to the previous single micro-fiber scaffold were obtained. The cells appear to spread along the axial direction on the scaffold and the crosssectional image (inserted) confirmed the cells are tightly enveloping the entire circumference of the scaffold. The successful formation of HDMECs-covered branched structures suggests our approach is highly feasible for the construction of the microvascular network in vitro.

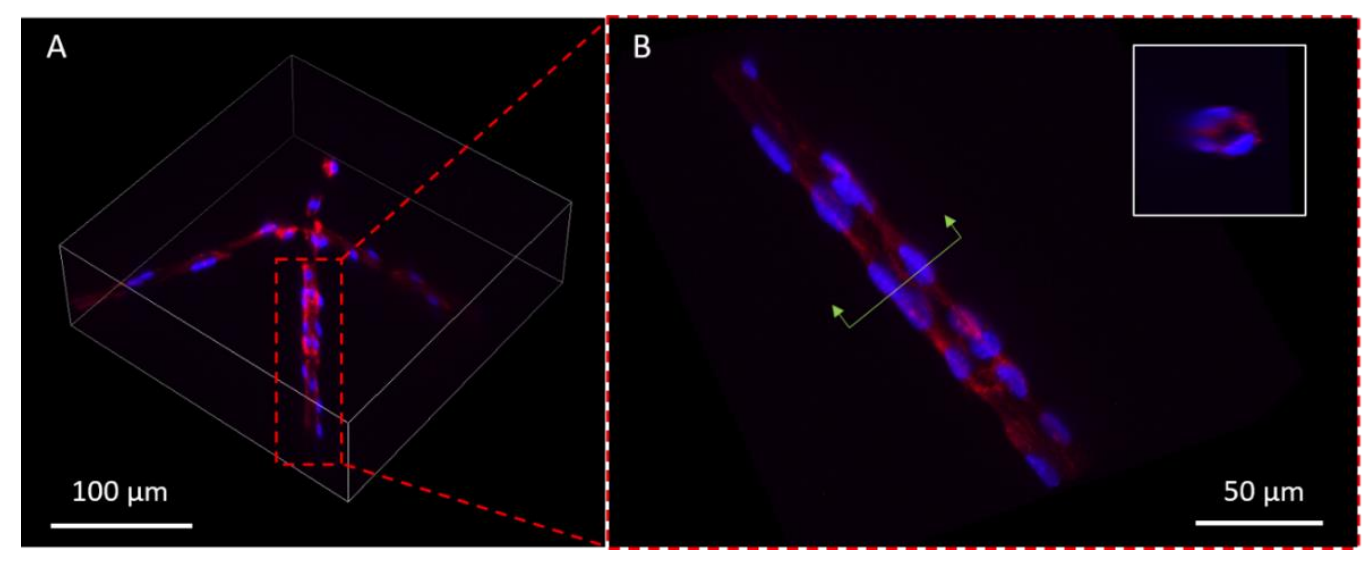

Figure 4. 35 A) 3D reconstruction confocal image of HDEMCs on PLA-PEG branched structure stained for the nuclei (blue) and actin cytoskeletons (red) at 20X magnification, B) partial enlargement of (A) with a cross-sectional image (insert) at $40 \mathrm{X}$ magnification

\subsection{Prevascularization of Hybrid Constructs}

We have introduced that collagen, fibrin, and Matrigel ${ }^{\mathrm{TM}}$ could be used as ECM to provide a three-dimensional environment that structurally supports cells and allows for a diffusion of nutrients. After we successfully obtained the cell- 
covered scaffold, we want to further encapsulate it into an ECM to get a vascularized sheet to allow cells to attach and maintain a lumen shape after the scaffold is degraded. To demonstrate this conceptual method, we embedded the cell-covered scaffolds into a $1 \mathrm{mg} / \mathrm{mL}$ Type I collagen hydrogel and culture the hybrid constructs for an additional three days. Figure 4.36A shows the hybrid structures attached on the acrylic frame (highlighted in the red dot square) after three days of culture. The vascularized sheet was a soft, semi-transparent, and moisture-absorbing film. After carefully cutting the four edges of the film, the vascularized sheet detached from the frame. However, the sheet immediately twisted and folded due to the internal tension (Figure 4.36C and F, pointed with the red arrow). Surprisingly, the sheet could unfold itself just by adding a few drops of PBS solution. As can be seen in Figure 4.36D-E, the vascularized sheet gradually extended itself under the buoyancy of the PBS solution. Eventually, the vascularized sheet completely unfolded itself to form a free-standing film that is floating in the PBS solution. In the classical "bottom-up" approach for the tissue engineering, the key idea is first to create intermediate "modular tissues" and then assemble them into the desired engineered tissue[208]. Guided by this strategy, we added another vascularized sheet into the system and it also unfolded automatically (Figure 4.36F-H). These two or even more two-dimensional sheets could be used as the "modular tissues" that are eventually stacked on each other to form more advanced three-dimensional architecture. Figure 4.36B shows the vascularized sheet after fixation and dehydration became dry and brittle. It is worth noting that after a series of graded ethanol washes, the sheet needed to be taken 
out from the frame and transferred to a PTFE substrate before it completely dried. Otherwise, the sheet will stick to the bottom of the wall plate and will be difficult to peel off.

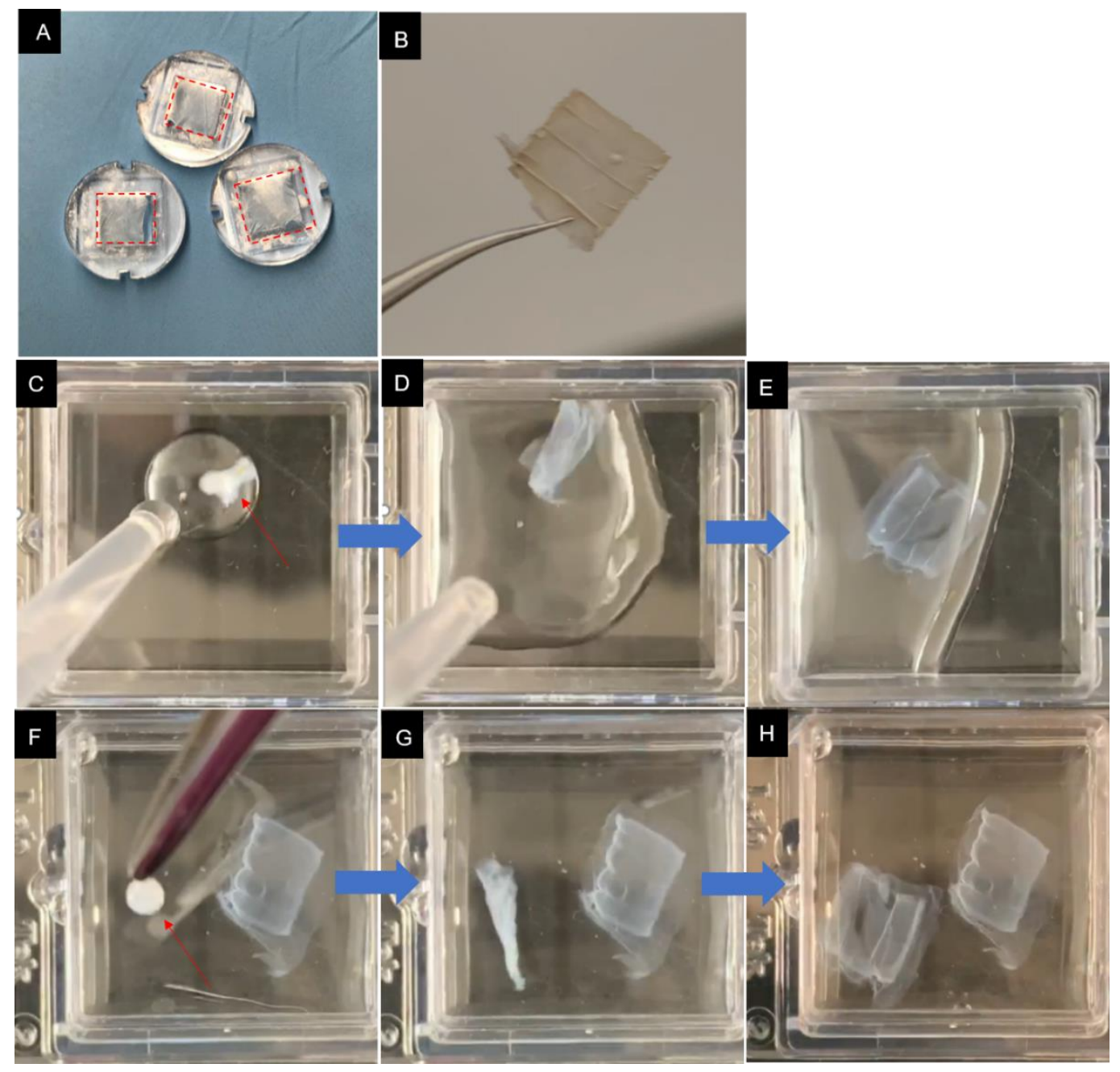

Figure 4. 36 Optical images of the vascularized sheets: A) on the custom acrylic frame, B) after fixation and dehydration, C-E) hybrid structure unfolded itself in the PBS solution, and F-H) two hybrid structures in the PBS solution. 
Using fluorescent staining, the inner structure of the vascularized sheet could be observed and the growth status of the cells in the ECM could be evaluated. As shown in Figure 4.37A-B, the HDMECs on both a single micro-fiber and a branched structure scaffold could be seen. Although the images are a bit hazy (may be caused by the scattering of the fluorescent light in the opaque collagen gel), we can still confirm that the HDMECs are growing along the axial direction of the scaffolds. No migrated cells near the scaffold were observed, indicating that the HDMECs were well attached on the scaffolds with the help of mechanical support and the diffusion of nutrients aided by the ECM. Figure 4.37C-D shows the 3D reconstruction confocal images of the hybrid structure. Notably, the inserted cross-sectional image in Figure 4.37C shows the cells were wrapping the circumference of the scaffold and the lumen shape was still maintained. Also, the cells in the $3 \mathrm{D}$ confocal images were observed to not migrate from the initial scaffold. To further evaluate the viability of the cells after they were encapsulated into the ECM, the live/dead assay was utilized and the results are shown in Figure 4.37E-F. One can see most of the cells on the scaffold were still alive. However, a few granule-shaped cells and dead cells could be observed, suggesting the cells may not last long on the scaffold. This behavior may be caused by contact inhibition, which was often found in the monolayer endothelial cells culture if there was no chemotactic or mechanical stimuli[209, 210]. Nevertheless, this approach has the possibility to create a directional microvascular network within the collagen hydrogel matrix. 


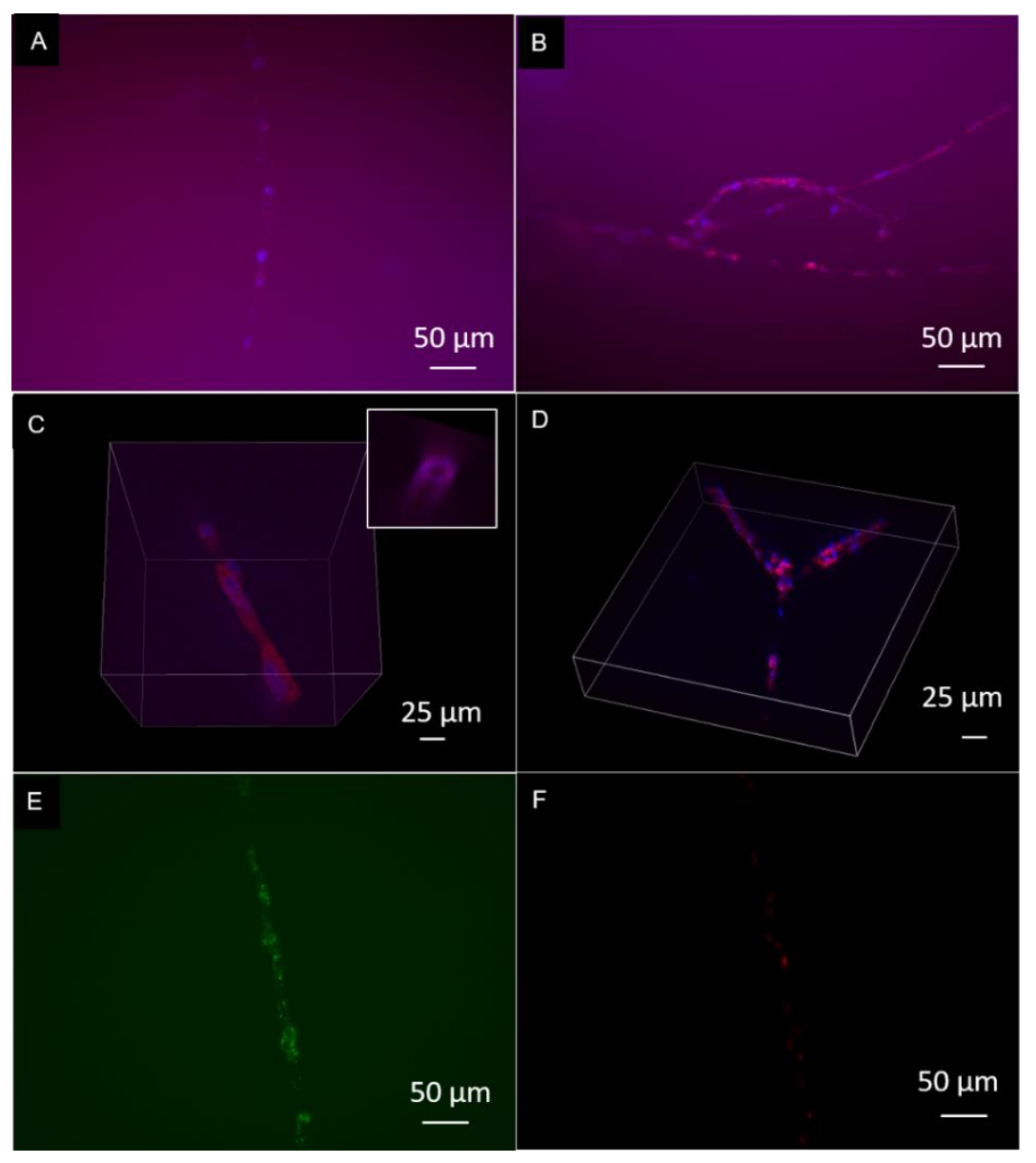

Figure 4. 37 Fluorescent images of a A) single micro-fiber and a B) branched structure stained for the nuclei (blue) and actin cytoskeletons (red); 3D reconstruction confocal images of a C) single micro-fiber and a B) branched stained for the nuclei (blue) and actin cytoskeletons (red); E-F) fluorescent images of the live/dead assay stained to highlight the live cells (Calcein AM, green) and dead cells (EthD-1, red).

To examine the microscopic morphology of the hybrid structure, the dehydrated sheet was cut perpendicular to the direction of the micro-fiber scaffold by a blade and visualized through the SEM (Figure 4.38A). Figure 4.38B presents a 106X magnification the SEM image to show the overall perspective of the cross- 
sectional cut. Two parallel micro-fiber scaffolds could be identified. Three distinctive spots were selected to further study the microstructures. The side view of the hybrid structure is shown in Figure 4.38C. We could see the dehydrated collagen network is composed of fiber bundles and small pores. The fibrous and porous microstructure could supply not only mechanical support, but also allow the diffusion of nutrients to the cells. Figure 4.38D shows the cross-sectional view of the collagen hydrogel film: the average thickness of the film is approximately 18 $\mu \mathrm{m}$. We can see the dehydrated film consists of several layers that are interconnected with small pores. The highly porous microstructure has been shown to play a critical role in cells proliferation and media diffusion. Figure $4.38 \mathrm{E}$ presents the cross-sectional image of the vascularized sheet. The circular shape of the scaffold, the fibrous nature of the ECM, and several pieces of cell debris could be seen from the incision. Figure $4.38 \mathrm{~F}$ is a sample where the cutting process accidentally broke the side of the collagen hydrogel. We could see more intact cells are attached on the scaffold. This image could further confirm that the vascularized sheet exhibits scaffold-cell-ECM interactions. From these images, there is still no evidence to suggest the scaffolds have degraded because the scaffolds are still tightly wrapped in the ECM. Also, the previous degradation experiments showed that after three days, an obvious mass change of the biopolymer will not be observed. 


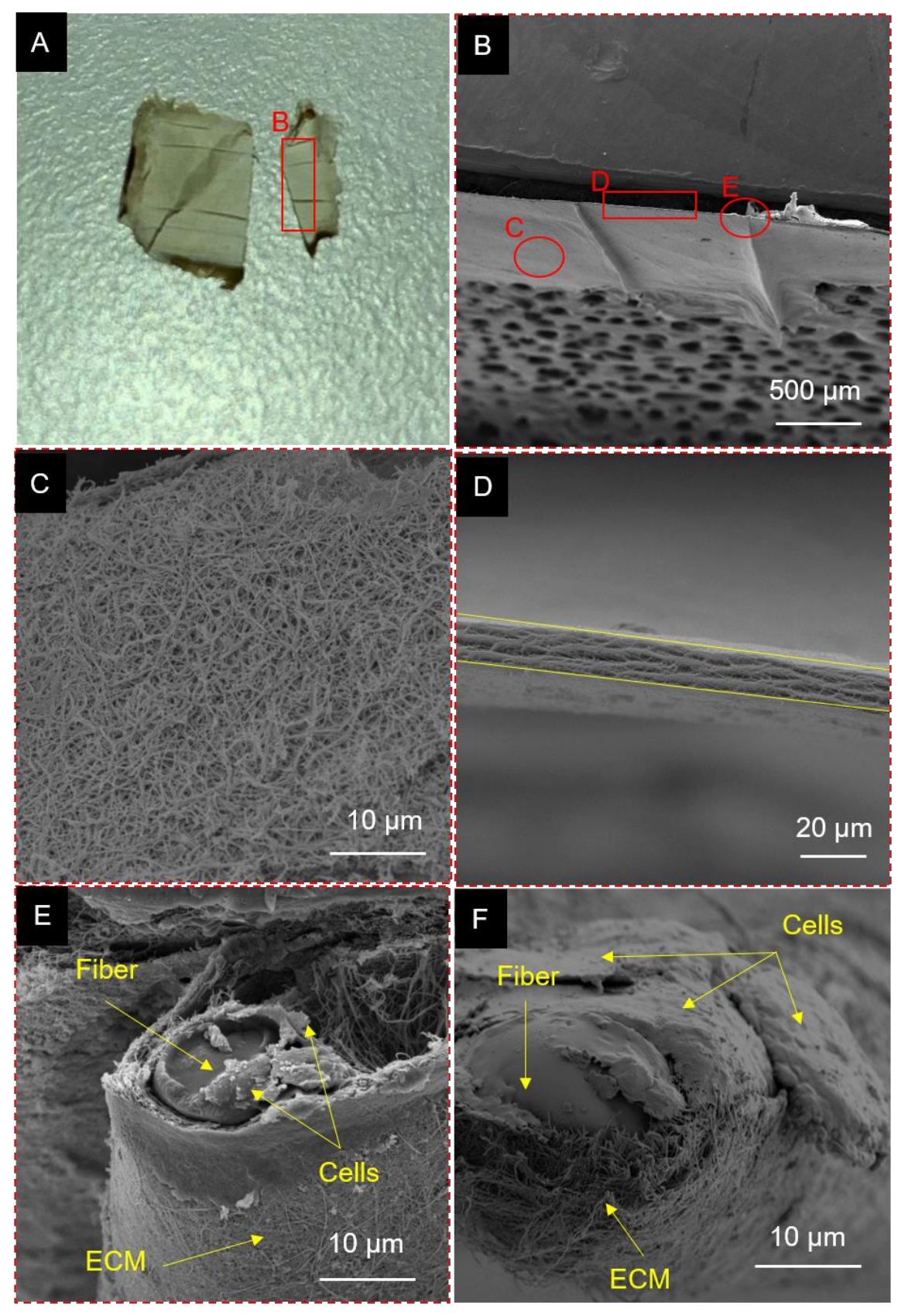

Figure 4. 38 A) Optical image of the dehydrated vascularized sheet after radial cutting. SEM images of B) an overview of the cross section (106X magnification), C) side view of the dehydrated ECM (4.39 KX magnification), D) cross-sectional view of the dehydrated ECM (1.98 KX magnification), and E, F) cross-sectional 
view of the vascularized sheet with scaffold-cell-ECM interaction $(6.0 \mathrm{KX}$ magnification)

In order to further study the scaffold degradation behavior inside of the ECM, we increased the culture time to two weeks. Figure 4.39 presents the crosssectional views of the vascularized sheet under different culture times. The upper row images (A1-A3) show several examples of the samples under three days of culture. Similar to the previous result, the circular-shaped micro-fiber scaffold (highlighted by the green dashed line) was closely covered by the collagen gel, and no space could be seen between them. Also, no visible HDMECs were observed in these views since the connection between the scaffold and collagen was very tight and the endothelial cells may not have been distinguishable due to the small cell thickness $(500 \mathrm{~nm})$. It is worth noting that most of the samples under two weeks of culturing were not observed to have obvious scaffold degradation. The lower images (B1-B3) show several representative examples of vascularized sheet that have aby indication of scaffold degradation. As can be seen, these micro-fiber scaffolds showed various cross-sectional shapes, suggesting they were experienced different degradation degree. For instance, the scaffold in Figure 4.39B-1 shows an irregular polygon-shaped scaffold and the lumen outline (highlighted in the red dashed line). The cavity on the scaffold surface may have been formed by the surface erosion degradation mechanism; the measured crosssectional area ratio of the scaffold and the lumen is approximately $82.3 \%$. In Figure 4.39B-2, a taper-shaped scaffold with a 44.5\% scaffold/lumen outline ratio. This decrease in ratio may suggest more scaffold materials degraded away in the 
same culturing environment. These differences in degradation may be caused by both the different diffusion rates within the complex collagen network and unknown interactions between the ECM, cells, and the scaffold. In Figure 4.39B-3, we observed a perfect lumen within the collagen hydrogel without any scaffold remaining. However, this result does not conform to the previous degradation test result in which the PLA-PEG $(75: 25)$ copolymer was not able to completely degrade in two weeks. We speculated three reasons as to why the scaffold degraded in this sample: (1) the scaffold broke or pulled away during the crosssectional cut; (2) the scaffold may have experienced bulk erosion and the debris was washed away by the media; (3) this sample was left in the desiccator for two weeks before taking SEM images and the vacuum environment may have caused the scaffold to detach from the dehydrated collagen gel. The future challenge involves better understanding the degradation mechanism of the biopolymer scaffold within the collagen hydrogel, being able to accelerate the hydrolyzation rate by adding a catalyst or finding a new material with a faster degradation rate to allow the scaffold to degrade completely in one week. 

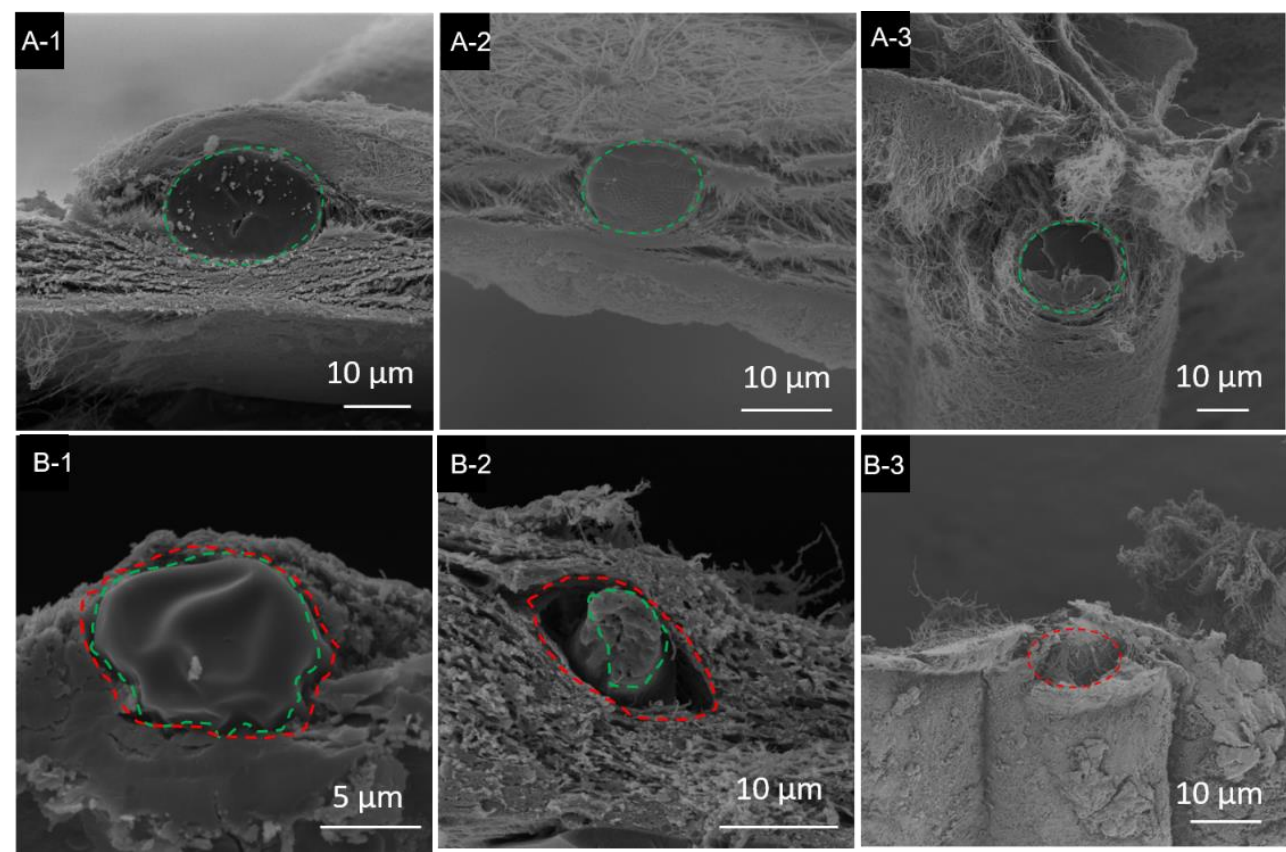

Figure 4. 39 SEM images of the cross-sectional view of the vascularized sheets after: A1-A3) three days culture, B1-B3) two weeks culture 


\section{CHAPTER 5: CONCLUSIONS AND FUTURE DIRECTIONS}

The capability to selectively produce microcirculatory vessels is critical to the emerging field of tissue engineering. In order to produce microvasculature, a scaffold is required to support and stimulate endothelial cell adhesion and growth. The primary goal of this project was to develop both a protocol for the construction of a precisely positioned, three-dimensional, suspended biopolymer scaffold with varying diameters and a conceptual scaffold-covering strategy to create physiological microvascular networks in vitro.

In this work, we extended the direct-write technique and adopted the 3-Axis robotic dispensing system developed by Dr. Scott Berry and Dr. Hanwen Yuan, who previously worked in our laboratory. Gelatin, PLGA, Gelatin/PLGA composites, PLA, PLA-PEG copolymers were successfully processed into precisely-positioned, suspended micro-fibers and branched structures. The geometries of the microfibers and the branched structures were successfully controlled during fabrication to match the microvascular-scale $(5-40 \mu \mathrm{m})$. We have demonstrated the ability to control the spatial orientation of the branched structures and the relative errors for the actual and design branching angles were less than $9 \%$. We also demonstrated the ability to develop the branched structures with varying diameters so that they could better mimic the real capillary system structure of arterioles, capillaries, and venules. Moreover, several complex structures such as web structure with multiintersections, parallel fiber array, and overlapping fibers with the orthotropic 
structure were also successfully fabricated by precisely controlling the dispensing tip to move between the predefined spatial spots.

In addition to fabricating the biopolymer micro-fibers, we expected to better understand the fiber formation mechanism behind the thinning dynamics of biopolymer solutions as well as determine what process factors affected the microfiber yield and diameter. The direct-write process was characterized by a single polymer/solvent system (gelatin/TFE) through the unbalanced four factors multilevel full factorial design of experiment. Biopolymer solutions' concentration, needle's inner diameter, feed rate, and fiber length were confirmed as the significant factors that could both affect fiber yield and diameters $(p=0.000$ for both). Next, a dimensional analysis was performed to reduce the 6 physical variables to 3 dimensionless parameters, followed by the employment of a linear regression analysis to generate an empirical model based on the dimensionless parameters. This empirical model revealed the correlation between the fiber diameter, polymer solution properties, and system process parameters. Then, the empirical model was validated by various biopolymer/solvents systems and the predicted and experimental data of micro-fibers diameters was compared. By excluding the out-of-boundary data, the mean absolute deviation was $4.30 \mu \mathrm{m}$, and the mean absolute percentage error was $38.22 \%$.

After considering the multi-factors of the direct-write yield, the biodegradation rate, and the endothelial cells adhesion ability and viability comprehensively, the PLA-PEG (75:25) copolymer was selected to be seeded with HDMECs. The optimized seeding conditions were as follow: (1) the bottom of the 
24-well plate was blocked by $0.01 \%$ BSA solution; (2) the scaffold was surface modified by $5 \mu \mathrm{g} / \mathrm{mL}$ fibronectin solution; (3) a seeding density of 80,000 cells $/ \mathrm{cm}^{2}$ was selected. The HDMECs cultured on suspended scaffolds were observed to be living on the surface of the three-dimensional branched structures and had an elongated shape with an aligned orientation. The 3D reconstructed confocal images confirmed the HDMECs proliferated both along the axis and around the circumference of the micro-fibers and to create a confluent monolayer of cells on the surface of the micro-fiber. The VE-cadherin expression of the HDMECs demonstrated the neighboring cells adhered to each other and formed cell-cell junctions.

The last step for the scaffold-covering strategy is to encapsulate the cellcovered scaffold into the ECM and let the scaffold degrade through hydrolysis. In this study, we successfully embedded the cell-covered scaffold into a collagen gel and cultured for three additional days. The fluorescent and confocal images showed the viable cells were still wrapped on the scaffold and maintained the cylinder-shaped monolayer. The cross-sectional SEM images confirmed the ECMcell-scaffold interactions. No visible scaffold degradation was observed after three days of culture, while a few samples showed obvious mass loss (17.7\% - 55.5\%) after two weeks of culture through the cross-sectional images. Although the scaffold was not completely degraded as we expected in this study, we demonstrate the ability to obtain a flexible and free-standing "modular tissue," which could be potentially assembled to a 3D microvascular network in the future work. 
In this dissertation, we developed the proof-of-concept scaffold-covering strategy to create a microvascular network with the direct-write scaffold method in vitro. Based on the present progress and current understanding of the formation mechanism of the microvasculature, the following recommendations are made with respect to future work:

(1) Develop a new biodegradable polymer with a faster degradation rate (less than a week) which could be direct-written as well. For the PLA-PEG copolymer, a polymerization catalyst such as stannous octoate could be used to prompt the reaction rate and increase the ester bond ratio, therefore accelerating the hydrolysis process[205].

(2) A co-culture system could have potential in the construction of microvascular systems, specifically enhancing the cell lumen integrity and viability. Supporting cells could include pericytes, vascular smooth muscle cells, and mesenchymal cell[211].

(3) Further experimentation should be conducted to gain insight into the utilization of specific growth factors such vascular endothelial growth factors, junctional proteins such as the vascular endothelial cadherin, and extracellular proteins such as EGF like domain 7 during the capillary system formation process[212].

(4) Manipulation of external forces-such as cyclic and static strain, as well as flow-induced shear stress-may help the HDMECs suppress apoptosis, enabling the cells to survive during the scaffold hydrolysis degradation. These 
external forces may also help to create a perfusable vessel and maintain the lumen integrity of the cell monolayer.

(5) An in vivo animal study will be beneficial to further evaluate the potential of "modular tissue" sheets developing into a three-dimensional functional microvascular network through angiogenesis. 


\section{REFERENCES}

[1] World Health Organization. "GLOBAL HEALTH ESTIMATES 2015 SUMMARY TABLES: GLOBAL DEATHS BY CAUSE, AGE AND SEX, 2000-2015," http://www.who.int/healthinfo/global burden disease/en/.

[2] A. Persidis, "Tissue engineering," Nature Biotechnology, vol. 17, pp. 508, 05/01/online, 1999.

[3] P. C. Simmonds, J. N. Primrose, J. L. Colquitt, O. J. Garden, G. J. Poston, and M. Rees, "Surgical resection of hepatic metastases from colorectal cancer: A systematic review of published studies," British Journal Of Cancer, vol. 94, pp. 982, 03/14/online, 2006.

[4] J. K. Maguire, M. F. Coscia, and M. H. Lynch, "FOREIGN-BODY REACTION TO POLYMERIC DEBRIS FOLLOWING TOTAL HIPARTHROPLASTY," Clinical Orthopaedics and Related Research, no. 216, pp. 213-223, Mar, 1987.

[5] Organ Procurement and Transplantation Network. "The organ shortage continues," April 9, 2018; https://optn.transplant.hrsa.gov/.

[6] J. P. Vacanti, M. A. Morse, W. M. Saltzman, A. J. Domb, A. Perez-Atayde, and R. Langer, "Selective cell transplantation using bioabsorbable artificial polymers as matrices," Journal of Pediatric Surgery, vol. 23, no. 1, pp. 3-9.

[7] R. Langer, and J. Vacanti, "Tissue engineering," Science, vol. 260, no. 5110, pp. 920-926, 1993.

[8] R. Langer, and J. Vacanti, "Advances in tissue engineering," Journal of Pediatric Surgery, vol. 51, no. 1, pp. 8-12, 2016/01/01/, 2016.

[9] P. Bajaj, R. M. Schweller, A. Khademhosseini, J. L. West, and R. Bashir, "3D Biofabrication Strategies for Tissue Engineering and Regenerative Medicine," Annual Review of Biomedical Engineering, vol. 16, no. 1, pp. 247-276, 2014/07/11, 2014.

[10] A. Jaklenec, A. Stamp, E. Deweerd, A. Sherwin, and R. Langer, "Progress in the Tissue Engineering and Stem Cell Industry "Are we there yet?"," Tissue Engineering Part B: Reviews, vol. 18, no. 3, pp. 155-166, 2012/06/01, 2012.

[11] C. D. Pritchard, J. R. Slotkin, D. Yu, H. Dai, M. S. Lawrence, R. T. Bronson, F. M. Reynolds, Y. D. Teng, E. J. Woodard, and R. S. Langer, "Establishing a model spinal cord injury in the African green monkey for the preclinical evaluation of biodegradable polymer scaffolds seeded with human neural stem cells," Journal of Neuroscience Methods, vol. 188, no. 2, pp. 258-269, 2010/05/15/, 2010.

[12] J. S. Miller, K. R. Stevens, M. T. Yang, B. M. Baker, D.-H. T. Nguyen, D. M. Cohen, E. Toro, A. A. Chen, P. A. Galie, and X. Yu, "Rapid casting of patterned vascular networks for perfusable engineered three-dimensional tissues," Nature materials, vol. 11, no. 9, pp. 768, 2012. 
[13] J. F. Hansbrough, C. Doré, and W. B. Hansbrough, "Clinical trials of a living dermal tissue replacement placed beneath meshed, split-thickness skin grafts on excised burn wounds," The Journal of burn care \& rehabilitation, vol. 13, no. 5, pp. 519-529, 1992.

[14] A. Atala, S. B. Bauer, S. Soker, J. J. Yoo, and A. B. Retik, "Tissueengineered autologous bladders for patients needing cystoplasty," The lancet, vol. 367, no. 9518, pp. 1241-1246, 2006.

[15] S. MacNeil, "Progress and opportunities for tissue-engineered skin," Nature, vol. 445, no. 7130, pp. 874, 2007.

[16] C. Clar, E. Cummins, L. McIntyre, S. Thomas, J. Lamb, L. Bain, P. Jobanputra, and N. Waugh, "Clinical and cost-effectiveness of autologous chondrocyte implantation for cartilage defects in knee joints: systematic review and economic evaluation," 2005.

[17] Y. Ikada, "Challenges in tissue engineering," Journal of the Royal Society Interface, vol. 3, no. 10, pp. 589-601, 2006.

[18] M. S. Chapekar, "Tissue engineering: challenges and opportunities," Journal of Biomedical Materials Research: An Official Journal of The Society for Biomaterials, The Japanese Society for Biomaterials, and The Australian Society for Biomaterials and the Korean Society for Biomaterials, vol. 53, no. 6, pp. 617-620, 2000.

[19] E. C. Novosel, C. Kleinhans, and P. J. Kluger, "Vascularization is the key challenge in tissue engineering," Advanced drug delivery reviews, vol. 63, no. 4-5, pp. 300-311, 2011.

[20] J. Rouwkema, N. C. Rivron, and C. A. van Blitterswijk, "Vascularization in tissue engineering," Trends in biotechnology, vol. 26, no. 8, pp. 434-441, 2008.

[21] E. A. Phelps, and A. J. García, "Engineering more than a cell: vascularization strategies in tissue engineering," Current opinion in biotechnology, vol. 21, no. 5, pp. 704-709, 2010.

[22] B. Vailhé, D. Vittet, and J.-J. Feige, "In vitro models of vasculogenesis and angiogenesis," Laboratory investigation, vol. 81, no. 4, pp. 439, 2001.

[23] W. Risau, "Mechanisms of angiogenesis," Nature, vol. 386, pp. 671, 04/17/online, 1997.

[24] T. Kucera, J. Eglinger, B. Strilic, and E. Lammert, "Vascular lumen formation from a cell biological perspective." p. 46.

[25] C. Khanna, and K. Hunter, "Modeling metastasis in vivo," Carcinogenesis, vol. 26, no. 3, pp. 513-523, 2005.

[26] A. G. Mikos, G. Sarakinos, M. D. Lyman, D. E. Ingber, J. P. Vacanti, and R. Langer, "Prevascularization of porous biodegradable polymers," Biotechnology and Bioengineering, vol. 42, no. 6, pp. 716-723, 1993.

[27] I. Montaño, C. Schiestl, J. Schneider, L. Pontiggia, J. Luginbühl, T. Biedermann, S. Böttcher-Haberzeth, E. Braziulis, M. Meuli, and E. Reichmann, "Formation of human capillaries in vitro: the engineering of prevascularized matrices," Tissue Engineering Part A, vol. 16, no. 1, pp. 269-282, 2009. 
[28] L. Tian, and S. C. George, "Biomaterials to prevascularize engineered tissues," Journal of cardiovascular translational research, vol. 4, no. 5, pp. 685, 2011.

[29] I. Sukmana, "Microvascular guidance: a challenge to support the development of vascularised tissue engineering construct," The Scientific World Journal, vol. 2012, 2012.

[30] S. Singh, B. M. Wu, and J. C. Dunn, "Accelerating vascularization in polycaprolactone scaffolds by endothelial progenitor cells," Tissue Engineering Part A, vol. 17, no. 13-14, pp. 1819-1830, 2011.

[31] I. K. Zervantonakis, S. K. Hughes-Alford, J. L. Charest, J. S. Condeelis, F. B. Gertler, and R. D. Kamm, "Three-dimensional microfluidic model for tumor cell intravasation and endothelial barrier function," Proceedings of the National Academy of Sciences, vol. 109, no. 34, pp. 13515-13520, 2012.

[32] X. Cui, and T. Boland, "Human microvasculature fabrication using thermal inkjet printing technology," Biomaterials, vol. 30, no. 31, pp. 6221-7, Oct, 2009.

[33] K. H. Wong, J. M. Chan, R. D. Kamm, and J. Tien, "Microfluidic models of vascular functions," Annual review of biomedical engineering, vol. 14, pp. 205-230, 2012.

[34] J. E. Hall, "Guyton and Hall textbook of medical physiology," Philadelphia, PA: Saunders Elsevier, pp. 107, 2011.

[35] F. H. M. J. L. N. E. F. Bartholomew, Fundamentals of Anatomy \& Physiology (11th Edition).

[36] P. Carmeliet, and R. K. Jain, "Angiogenesis in cancer and other diseases," nature, vol. 407, no. 6801, pp. 249, 2000.

[37] e. Kelly A. Young, . Anatomy \& Physiology (1st Edition): OpenStax College.

[38] E. N. Marieb, and K. Hoehn, Human anatomy \& physiology, Harlow: Pearson Education Limited, 2016.

[39] K. G. Birukov, J. R. Jacobson, A. A. Flores, S. Q. Ye, A. A. Birukova, A. D. Verin, and J. G. Garcia, "Magnitude-dependent regulation of pulmonary endothelial cell barrier function by cyclic stretch," American Journal of Physiology-Lung Cellular and Molecular Physiology, vol. 285, no. 4, pp. L785-L797, 2003.

[40] J. Ando, and K. Yamamoto, "Effects of shear stress and stretch on endothelial function," Antioxidants \& redox signaling, vol. 15, no. 5, pp. 1389-1403, 2011.

[41] Promocell. "Human Primary Cells," https://www.promocell.com/products/human-primary-cells/.

[42] N. Ferrara, "Role of vascular endothelial growth factor in the regulation of angiogenesis," Kidney international, vol. 56, no. 3, pp. 794-814, 1999.

[43] T. Rozario, and D. W. DeSimone, "The extracellular matrix in development and morphogenesis: a dynamic view," Developmental biology, vol. 341, no. 1, pp. 126-140, 2010. 
[44] H. Järveläinen, A. Sainio, M. Koulu, T. N. Wight, and R. Penttinen, "Extracellular matrix molecules: potential targets in pharmacotherapy," Pharmacological reviews, vol. 61, no. 2, pp. 198-223, 2009.

[45] C. Frantz, K. M. Stewart, and V. M. Weaver, "The extracellular matrix at a glance," Journal of Cell Science, vol. 123, no. 24, pp. 4195-4200, 12/01, 2010.

[46] L. Schaefer, and R. M. Schaefer, "Proteoglycans: from structural compounds to signaling molecules," Cell and tissue research, vol. 339, no. 1, pp. 237, 2010.

[47] F. T. Bosman, and I. Stamenkovic, "Functional structure and composition of the extracellular matrix," The Journal of Pathology: A Journal of the Pathological Society of Great Britain and Ireland, vol. 200, no. 4, pp. 423428, 2003.

[48] W. P. Daley, S. B. Peters, and M. Larsen, "Extracellular matrix dynamics in development and regenerative medicine," Journal of cell science, vol. 121, no. 3, pp. 255-264, 2008.

[49] B. Alberts, A. Johnson, J. Lewis, M. Raff, K. Roberts, and P. Walter, "Molecular biology of the cell: Reference edition," 2007.

[50] M. L. Smith, D. Gourdon, W. C. Little, K. E. Kubow, R. A. Eguiluz, S. LunaMorris, and V. Vogel, "Force-induced unfolding of fibronectin in the extracellular matrix of living cells," PLoS biology, vol. 5, no. 10, pp. e268, 2007.

[51] H. K. Kleinman, D. Philp, and M. P. Hoffman, "Role of the extracellular matrix in morphogenesis," Current opinion in biotechnology, vol. 14, no. 5, pp. 526-532, 2003.

[52] B. L. Hogan, and P. A. Kolodziej, "Organogenesis: molecular mechanisms of tubulogenesis," Nature Reviews Genetics, vol. 3, no. 7, pp. 513, 2002.

[53] R. Auerbach, R. Lewis, B. Shinners, L. Kubai, and N. Akhtar, "Angiogenesis assays: a critical overview," Clinical chemistry, vol. 49, no. 1, pp. 32-40, 2003.

[54] L. L. Bischel, E. W. Young, B. R. Mader, and D. J. Beebe, "Tubeless microfluidic angiogenesis assay with three-dimensional endothelial-lined microvessels," Biomaterials, vol. 34, no. 5, pp. 1471-7, Feb, 2013.

[55] F. J. O'brien, "Biomaterials \& scaffolds for tissue engineering," Materials today, vol. 14, no. 3, pp. 88-95, 2011.

[56] P. A. Gunatillake, and R. Adhikari, "Biodegradable synthetic polymers for tissue engineering," Eur Cell Mater, vol. 5, no. 1, pp. 1-16, 2003.

[57] A. B. Kutikov, and J. Song, "Biodegradable PEG-based amphiphilic block copolymers for tissue engineering applications," ACS biomaterials science \& engineering, vol. 1, no. 7, pp. 463-480, 2015.

[58] S.-B. Park, E. Lih, K.-S. Park, Y. K. Joung, and D. K. Han, "Biopolymerbased functional composites for medical applications," Progress in Polymer Science, vol. 68, pp. 77-105, 2017.

[59] K. Van de Velde, and P. Kiekens, "Biopolymers: overview of several properties and consequences on their applications," Polymer testing, vol. 21, no. 4, pp. 433-442, 2002. 
[60] M. Fittkau, P. Zilla, D. Bezuidenhout, M. Lutolf, P. Human, J. A. Hubbell, and N. Davies, "The selective modulation of endothelial cell mobility on RGD peptide containing surfaces by YIGSR peptides," Biomaterials, vol. 26, no. 2, pp. 167-174, 2005.

[61] S. Kumbar, C. Laurencin, and M. Deng, Natural and synthetic biomedical polymers: Newnes, 2014.

[62] D. Katti, S. Lakshmi, R. Langer, and C. Laurencin, "Toxicity, biodegradation and elimination of polyanhydrides," Advanced drug delivery reviews, vol. 54, no. 7, pp. 933-961, 2002.

[63] M. A. Rice, J. Sanchez-Adams, and K. S. Anseth, "Exogenously triggered, enzymatic degradation of photopolymerized hydrogels with polycaprolactone subunits: experimental observation and modeling of mass loss behavior," Biomacromolecules, vol. 7, no. 6, pp. 1968-1975, 2006.

[64] H. Naderi, M. M. Matin, and A. R. Bahrami, "Critical issues in tissue engineering: biomaterials, cell sources, angiogenesis, and drug delivery systems," Journal of biomaterials applications, vol. 26, no. 4, pp. 383-417, 2011.

[65] P. Basnett, and I. Roy, "Microbial production of biodegradable polymers and their role in cardiac stent development," Current Res Technol Educat Topics Applied Microbiol Microbial Biotechnol, pp. 405-1415, 2010.

[66] A. M. Tentori, and J. Jaworski, "Fabrication and Applications of Biological Fibers," Biodesign, vol. 2, pp. 69-80, 2014.

[67] C. H. Lee, A. Singla, and Y. Lee, "Biomedical applications of collagen," International journal of pharmaceutics, vol. 221, no. 1-2, pp. 1-22, 2001.

[68] J. L. Drury, and D. J. Mooney, "Hydrogels for tissue engineering: scaffold design variables and applications," Biomaterials, vol. 24, no. 24, pp. 43374351, 2003.

[69] B. Chevallay, and D. Herbage, "Collagen-based biomaterials as 3D scaffold for cell cultures: applications for tissue engineering and gene therapy," Medical and Biological Engineering and Computing, vol. 38, no. 2, pp. 211-218, 2000.

[70] U. Sampath, Y. Ching, C. Chuah, J. Sabariah, and P.-C. Lin, "Fabrication of porous materials from natural/synthetic biopolymers and their composites," Materials, vol. 9, no. 12, pp. 991, 2016.

[71] W. He, T. Yong, Z. W. Ma, R. Inai, W. E. Teo, and S. Ramakrishna, "Biodegradable polymer nanofiber mesh to maintain functions of endothelial cells," Tissue engineering, vol. 12, no. 9, pp. 2457-2466, 2006.

[72] J. A. Matthews, G. E. Wnek, D. G. Simpson, and G. L. Bowlin, "Electrospinning of collagen nanofibers," Biomacromolecules, vol. 3, no. 2, pp. 232-238, 2002.

[73] M. Li, M. J. Mondrinos, M. R. Gandhi, F. K. Ko, A. S. Weiss, and P. I. Lelkes, "Electrospun protein fibers as matrices for tissue engineering," Biomaterials, vol. 26, no. 30, pp. 5999-6008, 2005. 
[74] M. Shayegan, N. Rezaei, N. H. Lam, T. Altindal, A. Wieczorek, and N. R. Forde, "Probing multiscale mechanics of collagen with optical tweezers." p. $88101 \mathrm{P}$.

[75] C. Deng, F. Li, M. Griffith, M. Ruel, and E. J. Suuronen, "Application of Chitosan-Based Biomaterials for Blood Vessel Regeneration." pp. 138146.

[76] R. Hejazi, and M. Amiji, "Chitosan-based gastrointestinal delivery systems," Journal of controlled release, vol. 89, no. 2, pp. 151-165, 2003.

[77] R. Bhatt, and M. Jaffe, "Biopolymers in Medical Implants," Excipient Applications in Formulation Design and Drug Delivery, pp. 311-348: Springer, 2015.

[78] R. Lieder, M. Darai, G. Örlygsson, and O. E. Sigurjonsson, "Solution casting of chitosan membranes for in vitro evaluation of bioactivity," Biological procedures online, vol. 15, no. 1, pp. 11, 2013.

[79] M. Z. Albanna, T. H. Bou-Akl, O. Blowytsky, H. L. Walters III, and H. W. Matthew, "Chitosan fibers with improved biological and mechanical properties for tissue engineering applications," Journal of the mechanical behavior of biomedical materials, vol. 20, pp. 217-226, 2013.

[80] C.-H. Yeh, P.-W. Lin, and Y.-C. Lin, "Chitosan microfiber fabrication using a microfluidic chip and its application to cell cultures," Microfluidics and Nanofluidics, vol. 8, no. 1, pp. 115, 2010.

[81] J. Venkatesan, I. Bhatnagar, P. Manivasagan, K.-H. Kang, and S.-K. Kim, "Alginate composites for bone tissue engineering: a review," International journal of biological macromolecules, vol. 72, pp. 269-281, 2015.

[82] J. Sun, and $\mathrm{H}$. Tan, "Alginate-based biomaterials for regenerative medicine applications," Materials, vol. 6, no. 4, pp. 1285-1309, 2013.

[83] J. Kalisky, J. Raso, C. Rigothier, M. Rémy, R. Siadous, R. Bareille, J.-C. Fricain, J. Amedée-Vilamitjana, H. Oliveira, and R. Devillard, "An easy-touse and versatile method for building cell-laden microfibres," Scientific reports, vol. 6, pp. 33328, 2016.

[84] E. K. Yim, A. C. Wan, C. Le Visage, I.-C. Liao, and K. W. Leong, "Proliferation and differentiation of human mesenchymal stem cell encapsulated in polyelectrolyte complexation fibrous scaffold," Biomaterials, vol. 27, no. 36, pp. 6111-6122, 2006.

[85] S.-J. Shin, J.-Y. Park, J.-Y. Lee, H. Park, Y.-D. Park, K.-B. Lee, C.-M. Whang, and S.-H. Lee, "'On the fly" continuous generation of alginate fibers using a microfluidic device," Langmuir, vol. 23, no. 17, pp. 91049108, 2007.

[86] Y. Cheng, F. Zheng, J. Lu, L. Shang, Z. Xie, Y. Zhao, Y. Chen, and Z. Gu, "Bioinspired multicompartmental microfibers from microfluidics," Advanced Materials, vol. 26, no. 30, pp. 5184-5190, 2014.

[87] E. Kang, Y. Y. Choi, S. K. Chae, J. H. Moon, J. Y. Chang, and S. H. Lee, "Microfluidic spinning of flat alginate fibers with grooves for cell-aligning scaffolds," Advanced materials, vol. 24, no. 31, pp. 4271-4277, 2012.

[88] T. Takei, S. Sakai, T. Yokonuma, H. Ijima, and K. Kawakami, "Fabrication of artificial endothelialized tubes with predetermined three-dimensional 
configuration from flexible cell-enclosing alginate fibers," Biotechnology progress, vol. 23, no. 1, pp. 182-186, 2007.

[89] R. Gaetani, P. A. Doevendans, C. H. Metz, J. Alblas, E. Messina, A. Giacomello, and J. P. Sluijter, "Cardiac tissue engineering using tissue printing technology and human cardiac progenitor cells," Biomaterials, vol. 33, no. 6, pp. 1782-1790, 2012.

[90] T. J. Sill, and H. A. von Recum, "Electrospinning: applications in drug delivery and tissue engineering," Biomaterials, vol. 29, no. 13, pp. 19892006, 2008.

[91] S. Li, "Hydrolytic degradation characteristics of aliphatic polyesters derived from lactic and glycolic acids," Journal of Biomedical Materials Research: An Official Journal of The Society for Biomaterials, The Japanese Society for Biomaterials, and The Australian Society for Biomaterials, vol. 48, no. 3, pp. 342-353, 1999.

[92] A. Hadjizadeh, C. J. Doillon, and P. Vermette, "Bioactive polymer fibers to direct endothelial cell growth in a three-dimensional environment," Biomacromolecules, vol. 8, no. 3, pp. 864-873, 2007.

[93] X. Huang, S. Zauscher, B. Klitzman, G. A. Truskey, W. M. Reichert, D. J. Kenan, and M. W. Grinstaff, "Peptide interfacial biomaterials improve endothelial cell adhesion and spreading on synthetic polyglycolic acid materials," Annals of biomedical engineering, vol. 38, no. 6, pp. 19651976, 2010.

[94] M. Jamshidian, E. A. Tehrany, M. Imran, M. Jacquot, and S. Desobry, "Poly-lactic acid: production, applications, nanocomposites, and release studies," Comprehensive Reviews in Food Science and Food Safety, vol. 9, no. 5, pp. 552-571, 2010.

[95] D. Garlotta, "A literature review of poly (lactic acid)," Journal of Polymers and the Environment, vol. 9, no. 2, pp. 63-84, 2001.

[96] R. A. Auras, L.-T. Lim, S. E. Selke, and H. Tsuji, Poly (lactic acid): synthesis, structures, properties, processing, and applications: John Wiley \& Sons, 2011.

[97] P. B. Maurus, and C. C. Kaeding, "Bioabsorbable implant material review," Operative Techniques in Sports Medicine, vol. 12, no. 3, pp. 158-160, 2004.

[98] M. Charles-Harris, M. A. Koch, M. Navarro, D. Lacroix, E. Engel, and J. A. Planell, "A PLA/calcium phosphate degradable composite material for bone tissue engineering: an in vitro study," Journal of Materials Science: Materials in Medicine, vol. 19, no. 4, pp. 1503-1513, 2008.

[99] W. Ryu, S. W. Min, K. E. Hammerick, M. Vyakarnam, R. S. Greco, F. B. Prinz, and R. J. Fasching, "The construction of three-dimensional microfluidic scaffolds of biodegradable polymers by solvent vapor based bonding of micro-molded layers," Biomaterials, vol. 28, no. 6, pp. 11741184, 2007.

[100] T. Yokota, H. Ichikawa, G. Matsumiya, T. Kuratani, T. Sakaguchi, S. Iwai, Y. Shirakawa, K. Torikai, A. Saito, and E. Uchimura, "In situ tissue regeneration using a novel tissue-engineered, small-caliber vascular graft 
without cell seeding," The Journal of thoracic and cardiovascular surgery, vol. 136, no. 4, pp. 900-907, 2008.

[101] X. Yuan, A. F. Mak, K. Kwok, B. K. Yung, and K. Yao, "Characterization of poly (L-lactic acid) fibers produced by melt spinning," Journal of Applied Polymer Science, vol. 81, no. 1, pp. 251-260, 2001.

[102] Q. Zhou, J. Xie, M. Bao, H. Yuan, Z. Ye, X. Lou, and Y. Zhang, "Engineering aligned electrospun PLLA microfibers with nano-porous surface nanotopography for modulating the responses of vascular smooth muscle cells," Journal of Materials Chemistry B, vol. 3, no. 21, pp. 44394450, 2015.

[103] R. Hufenus, F. A. Reifler, K. Maniura-Weber, A. Spierings, and M. Zinn, "Biodegradable bicomponent fibers from renewable sources: melt-spinning of poly (lactic acid) and poly [(3-hydroxybutyrate)-co-(3-hydroxyvalerate)]," Macromolecular Materials and Engineering, vol. 297, no. 1, pp. 75-84, 2012.

[104] D. Gilding, and A. Reed, "Biodegradable polymers for use in surgerypolyglycolic/poly (actic acid) homo-and copolymers: 1," Polymer, vol. 20, no. 12 , pp. 1459-1464, 1979.

[105] J. C. Middleton, and A. J. Tipton, "Synthetic biodegradable polymers as orthopedic devices," Biomaterials, vol. 21, no. 23, pp. 2335-2346, 2000.

[106] H.-J. Sung, C. Meredith, C. Johnson, and Z. S. Galis, "The effect of scaffold degradation rate on three-dimensional cell growth and angiogenesis," Biomaterials, vol. 25, no. 26, pp. 5735-5742, 2004.

[107] E. Chevalier, D. Chulia, C. Pouget, and M. Viana, "Fabrication of porous substrates: a review of processes using pore forming agents in the biomaterial field," Journal of Pharmaceutical Sciences, vol. 97, no. 3, pp. 1135-1154, 2008.

[108] C. M. Hwang, A. Khademhosseini, Y. Park, K. Sun, and S.-H. Lee, "Microfluidic chip-based fabrication of PLGA microfiber scaffolds for tissue engineering," Langmuir, vol. 24, no. 13, pp. 6845-6851, 2008.

[109] K. D. Nelson, A. Romero, P. Waggoner, B. Crow, A. Borneman, and G. M. Smith, "Technique paper for wet-spinning poly (L-lactic acid) and poly (DLlactide-co-glycolide) monofilament fibers," Tissue engineering, vol. 9, no. 6, pp. 1323-1330, 2003.

[110] X. Zong, H. Bien, C.-Y. Chung, L. Yin, D. Fang, B. S. Hsiao, B. Chu, and E. Entcheva, "Electrospun fine-textured scaffolds for heart tissue constructs," Biomaterials, vol. 26, no. 26, pp. 5330-5338, 2005.

[111] G. Vozzi, C. Flaim, A. Ahluwalia, and S. Bhatia, "Fabrication of PLGA scaffolds using soft lithography and microsyringe deposition," Biomaterials, vol. 24, no. 14, pp. 2533-2540, 2003.

[112] S. I. Jeong, S. Y. Kim, S. K. Cho, M. S. Chong, K. S. Kim, H. Kim, S. B. Lee, and Y. M. Lee, "Tissue-engineered vascular grafts composed of marine collagen and PLGA fibers using pulsatile perfusion bioreactors," Biomaterials, vol. 28, no. 6, pp. 1115-1122, 2007.

[113] C. Xu, R. Inai, M. Kotaki, and S. Ramakrishna, "Electrospun nanofiber fabrication as synthetic extracellular matrix and its potential for vascular 
tissue engineering," Tissue engineering, vol. 10, no. 7-8, pp. 1160-1168, 2004.

[114] M. Labet, and W. Thielemans, "Synthesis of polycaprolactone: a review," Chemical Society Reviews, vol. 38, no. 12, pp. 3484-3504, 2009.

[115] E. S. Fioretta, M. Simonet, A. I. Smits, F. P. Baaijens, and C. V. Bouten, "Differential response of endothelial and endothelial colony forming cells on electrospun scaffolds with distinct microfiber diameters,"

Biomacromolecules, vol. 15, no. 3, pp. 821-829, 2014.

[116] J. Zhang, L. Wang, M. Zhu, L. Wang, N. Xiao, and D. Kong, "Wet-spun poly ( $\varepsilon$-caprolactone) microfiber scaffolds for oriented growth and infiltration of smooth muscle cells," Materials Letters, vol. 132, pp. 59-62, 2014.

[117] S. Park, B.-K. Lee, M. Na, and D. Kim, "Melt-spun shaped fibers with enhanced surface effects: fiber fabrication, characterization and application to woven scaffolds," Acta biomaterialia, vol. 9, no. 8, pp. 77197726, 2013.

[118] S. M. Berry, S. P. Warren, D. A. Hilgart, A. T. Schworer, S. Pabba, A. S. Gobin, R. W. Cohn, and R. S. Keynton, "Endothelial cell scaffolds generated by 3D direct writing of biodegradable polymer microfibers," Biomaterials, vol. 32, no. 7, pp. 1872-1879, 2011.

[119] M. Williamson, E. Adams, and A. Coombes, "Cell attachment and proliferation on novel polycaprolactone fibres having application in soft tissue engineering," European Cells and Materials, vol. 4, pp. 62-63, 2002.

[120] Q. P. Pham, U. Sharma, and A. G. Mikos, "Electrospinning of polymeric nanofibers for tissue engineering applications: a review," Tissue engineering, vol. 12, no. 5, pp. 1197-1211, 2006.

[121] X. Hu, S. Liu, G. Zhou, Y. Huang, Z. Xie, and X. Jing, "Electrospinning of polymeric nanofibers for drug delivery applications," Journal of controlled release, vol. 185, pp. 12-21, 2014.

[122] M. F. Ashby, "Materials selection in mechanical design," MRS Bull, vol. 30, no. 12, pp. 995, 2005.

[123] Y. Yang, D. Seol, H. Kim, M. Cho, and S. Lee, "Composite fibrin and collagen scaffold to enhance tissue regeneration and angiogenesis," Current Applied Physics, vol. 7, pp. e103-e107, 2007.

[124] J. S. Park, J. B. Choi, S. Y. Jo, Y. M. Lim, H. J. Gwon, M. S. Khil, and Y. C. Nho, "Characterization and structure analysis of PLGA/collagen nanofibrous membranes by electrospinning," Journal of Applied Polymer Science, vol. 125, no. S2, pp. E595-E603, 2012.

[125] S. Vaezifar, S. Razavi, M. A. Golozar, H. Z. Esfahani, M. Morshed, and S. Karbasi, "Characterization of PLGA/chitosan electrospun nanobiocomposite fabricated by two different methods," International Journal of Polymeric Materials and Polymeric Biomaterials, vol. 64, no. 2, pp. 64-75, 2015.

[126] S. Ali Akbari Ghavimi, M. H. Ebrahimzadeh, M. Solati-Hashjin, and N. A. Abu Osman, "Polycaprolactone/starch composite: Fabrication, structure, 
properties, and applications," Journal of biomedical materials research Part A, vol. 103, no. 7, pp. 2482-2498, 2015.

[127] G. Wang, X. Hu, W. Lin, C. Dong, and H. Wu, "Electrospun PLGA-silk fibroin-collagen nanofibrous scaffolds for nerve tissue engineering," In Vitro Cellular \& Developmental Biology-Animal, vol. 47, no. 3, pp. 234240, 2011.

[128] M. A. Slivka, N. C. Leatherbury, K. Kieswetter, and G. G. Niederauer, "Porous, resorbable, fiber-reinforced scaffolds tailored for articular cartilage repair," Tissue engineering, vol. 7, no. 6, pp. 767-780, 2001.

[129] A. Hokugo, T. Takamoto, and Y. Tabata, "Preparation of hybrid scaffold from fibrin and biodegradable polymer fiber," Biomaterials, vol. 27, no. 1, pp. 61-67, 2006.

[130] M. Norouzi, I. Shabani, H. H. Ahvaz, and M. Soleimani, "PLGA/gelatin hybrid nanofibrous scaffolds encapsulating EGF for skin regeneration," Journal of Biomedical Materials Research Part A, vol. 103, no. 7, pp. 2225-2235, 2015.

[131] M. A. Daniele, K. Radom, F. S. Ligler, and A. A. Adams, "Microfluidic fabrication of multiaxial microvessels via hydrodynamic shaping," $R S C$ Advances, vol. 4, no. 45, pp. 23440-23446, 2014.

[132] M. Akbari, A. Tamayol, N. Annabi, D. Juncker, and A. Khademhosseini, "Microtechnologies in the fabrication of fibers for tissue engineering," 2014.

[133] A. Tamayol, M. Akbari, N. Annabi, A. Paul, A. Khademhosseini, and D. Juncker, "Fiber-based tissue engineering: progress, challenges, and opportunities," Biotechnology advances, vol. 31, no. 5, pp. 669-687, 2013.

[134] L. M. Bellan, S. P. Singh, P. W. Henderson, T. J. Porri, H. G. Craighead, and J. A. Spector, "Fabrication of an artificial 3-dimensional vascular network using sacrificial sugar structures," Soft Matter, vol. 5, no. 7, pp. 1354-1357, 2009.

[135] D. H. Reneker, and I. Chun, "Nanometre diameter fibres of polymer, produced by electrospinning," Nanotechnology, vol. 7, no. 3, pp. 216, 1996.

[136] M. Deng, R. James, C. T. Laurencin, and S. G. Kumbar, "Nanostructured polymeric scaffolds for orthopaedic regenerative engineering," IEEE transactions on nanobioscience, vol. 11, no. 1, pp. 3-14, 2012.

[137] N. Bhardwaj, and S. C. Kundu, "Electrospinning: a fascinating fiber fabrication technique," Biotechnology advances, vol. 28, no. 3, pp. 325347, 2010.

[138] S. Zarkoob, R. Eby, D. H. Reneker, S. D. Hudson, D. Ertley, and W. W. Adams, "Structure and morphology of electrospun silk nanofibers," Polymer, vol. 45, no. 11, pp. 3973-3977, 2004.

[139] E. Zussman, "Encapsulation of cells within electrospun fibers," Polymers for Advanced Technologies, vol. 22, no. 3, pp. 366-371, 2011.

[140] Y. You, B. M. Min, S. J. Lee, T. S. Lee, and W. H. Park, "In vitro degradation behavior of electrospun polyglycolide, polylactide, and poly 
(lactide-co-glycolide)," Journal of Applied Polymer Science, vol. 95, no. 2, pp. 193-200, 2005.

[141] J. Xie, X. Li, and Y. Xia, "Putting electrospun nanofibers to work for biomedical research," Macromolecular rapid communications, vol. 29, no. 22, pp. 1775-1792, 2008.

[142] P. D. Dalton, N. T. Joergensen, J. Groll, and M. Moeller, "Patterned melt electrospun substrates for tissue engineering," Biomedical Materials, vol. 3, no. 3, pp. 034109, 2008.

[143] S. Zhang, X. Liu, S. F. Barreto-Ortiz, Y. Yu, B. P. Ginn, N. A. DeSantis, D. L. Hutton, W. L. Grayson, F.-Z. Cui, and B. A. Korgel, "Creating polymer hydrogel microfibres with internal alignment via electrical and mechanical stretching," Biomaterials, vol. 35, no. 10, pp. 3243-3251, 2014.

[144] M. Santos, K. Tuzlakoglu, M. Gomes, S. Fuchs, R. Unger, E. Piskin, R. Reis, and C. Kirkpatrick, "Nano-and micro-fiber combined scaffolds: an innovative design for improving endothelial cell migration in bone tissue engineering approaches." pp. 986-987.

[145] A. K. Ekaputra, G. D. Prestwich, S. M. Cool, and D. W. Hutmacher, "Combining electrospun scaffolds with electrosprayed hydrogels leads to three-dimensional cellularization of hybrid constructs,"

Biomacromolecules, vol. 9, no. 8, pp. 2097-2103, 2008.

[146] C. Xu, R. Inai, M. Kotaki, and S. Ramakrishna, "Aligned biodegradable nanofibrous structure: a potential scaffold for blood vessel engineering," Biomaterials, vol. 25, no. 5, pp. 877-886, 2004.

[147] Y. Zhu, Y. Cao, J. Pan, and Y. Liu, "Macro-alignment of electrospun fibers for vascular tissue engineering," Journal of Biomedical Materials Research Part B: Applied Biomaterials: An Official Journal of The Society for Biomaterials, The Japanese Society for Biomaterials, and The Australian Society for Biomaterials and the Korean Society for Biomaterials, vol. 92, no. 2, pp. 508-516, 2010.

[148] I. K. Kwon, and T. Matsuda, "Co-electrospun nanofiber fabrics of poly (Llactide-co- $\varepsilon$-caprolactone) with type I collagen or heparin," Biomacromolecules, vol. 6, no. 4, pp. 2096-2105, 2005.

[149] S. F. Barreto-Ortiz, J. Fradkin, J. Eoh, J. Trivero, M. Davenport, B. Ginn, H.-Q. Mao, and S. Gerecht, "Fabrication of 3-dimensional multicellular microvascular structures," The FASEB Journal, vol. 29, no. 8, pp. 33023314, 2015.

[150] J. Cheng, Y. Jun, J. Qin, and S.-H. Lee, "Electrospinning versus microfluidic spinning of functional fibers for biomedical applications," Biomaterials, vol. 114, pp. 121-143, 2017.

[151] W. Jeong, J. Kim, S. Kim, S. Lee, G. Mensing, and D. J. Beebe, "Hydrodynamic microfabrication via "on the fly" photopolymerization of microscale fibers and tubes," Lab on a Chip, vol. 4, no. 6, pp. 576-580, 2004.

[152] B. G. Chung, K.-H. Lee, A. Khademhosseini, and S.-H. Lee, "Microfluidic fabrication of microengineered hydrogels and their application in tissue engineering," Lab on a Chip, vol. 12, no. 1, pp. 45-59, 2012. 
[153] J. Su, Y. Zheng, and H. Wu, "Generation of alginate microfibers with a roller-assisted microfluidic system," Lab on a Chip, vol. 9, no. 7, pp. 9961001, 2009.

[154] X. Wang, Q. Sun, and J. Pei, "Microfluidic-Based 3D Engineered Microvascular Networks and Their Applications in Vascularized Microtumor Models," Micromachines, vol. 9, no. 10, pp. 493, 2018.

[155] D. Wei, J. Sun, J. Bolderson, M. Zhong, M. J. Dalby, M. Cusack, H. Yin, H. Fan, and X. Zhang, "Continuous fabrication and assembly of spatial cellladen fibers for a tissue-like construct via a photolithographic-based microfluidic chip," ACS applied materials \& interfaces, vol. 9, no. 17, pp. 14606-14617, 2017.

[156] R. Sato, and H. Onoe, "Fiber-shaped artificial tissue with microvascular networks for bottom-up tissue reconstruction." pp. 247-250.

[157] Y.-T. Tung, C.-C. Chang, J.-C. Ju, and G.-J. Wang, "Fabrication of a reticular poly (lactide-co-glycolide) cylindrical scaffold for the in vitro development of microvascular networks," Science and Technology of advanced MaTerialS, vol. 18, no. 1, pp. 163-171, 2017.

[158] Y. Tian, J. Wang, and L. Wang, "Microfluidic Fabrication of Bioinspired Cavity-Microfibers for 3D Scaffolds," ACS applied materials \& interfaces, vol. 10, no. 35, pp. 29219-29226, 2018.

[159] K. H. Lee, S. J. Shin, Y. Park, and S. H. Lee, "Synthesis of cell-laden alginate hollow fibers using microfluidic chips and microvascularized tissue-engineering applications," Small, vol. 5, no. 11, pp. 1264-1268, 2009.

[160] G.-S. Lee, J.-H. Park, U. S. Shin, and H.-W. Kim, "Direct deposited porous scaffolds of calcium phosphate cement with alginate for drug delivery and bone tissue engineering," Acta biomaterialia, vol. 7, no. 8, pp. 3178-3186, 2011.

[161] D. Puppi, D. Dinucci, C. Bartoli, C. Mota, C. Migone, F. Dini, G. Barsotti, F. Carlucci, and F. Chiellini, "Development of 3D wet-spun polymeric scaffolds loaded with antimicrobial agents for bone engineering," Journal of Bioactive and Compatible Polymers, vol. 26, no. 5, pp. 478-492, 2011.

[162] D. Puppi, C. Mota, M. Gazzarri, D. Dinucci, A. Gloria, M. Myrzabekova, L. Ambrosio, and F. Chiellini, "Additive manufacturing of wet-spun polymeric scaffolds for bone tissue engineering," Biomedical microdevices, vol. 14, no. 6, pp. 1115-1127, 2012.

[163] W. Qiu, W. Teng, J. Cappello, and X. Wu, "Wet-spinning of recombinant silk-elastin-like protein polymer fibers with high tensile strength and high deformability," Biomacromolecules, vol. 10, no. 3, pp. 602-608, 2009.

[164] B. Xu, L. Du, J. Zhang, M. Zhu, S. Ji, Y. Zhang, D. Kong, X. Ma, Q. Yang, and L. Wang, "Circumferentially oriented microfiber scaffold prepared by wet-spinning for tissue engineering of annulus fibrosus," RSC Advances, vol. 5, no. 53, pp. 42705-42713, 2015.

[165] B. R. Lee, K. H. Lee, E. Kang, D.-S. Kim, and S.-H. Lee, "Microfluidic wet spinning of chitosan-alginate microfibers and encapsulation of HepG2 cells in fibers," Biomicrofluidics, vol. 5, no. 2, pp. 022208, 2011. 
[166] F. Pati, B. Adhikari, and S. Dhara, "Development of chitosantripolyphosphate non-woven fibrous scaffolds for tissue engineering application," Journal of Materials Science: Materials in Medicine, vol. 23, no. 4, pp. 1085-1096, 2012.

[167] J. B. Lee, X. Wang, S. Faley, B. Baer, D. A. Balikov, H. J. Sung, and L. M. Bellan, "Development of 3D microvascular networks within gelatin hydrogels using thermoresponsive sacrificial microfibers," Advanced healthcare materials, vol. 5, no. 7, pp. 781-785, 2016.

[168] S. Kew, J. Gwynne, D. Enea, M. Abu-Rub, A. Pandit, D. Zeugolis, R. Brooks, N. Rushton, S. Best, and R. Cameron, "Regeneration and repair of tendon and ligament tissue using collagen fibre biomaterials," Acta Biomaterialia, vol. 7, no. 9, pp. 3237-3247, 2011.

[169] A. Charuchinda, R. Molloy, J. Siripitayananon, N. Molloy, and M. Sriyai, "Factors influencing the small-scale melt spinning of poly ( $\varepsilon$-caprolactone) monofilament fibres," Polymer international, vol. 52, no. 7, pp. 1175-1181, 2003.

[170] E. P. G. Gohl, and L. D. Vilensky, Textile science: Longman Cheshire, 1983.

[171] Q. Lu, A. Simionescu, and N. Vyavahare, "Novel capillary channel fiber scaffolds for guided tissue engineering," Acta Biomaterialia, vol. 1, no. 6, pp. 607-614, 2005.

[172] K. D. Sinclair, K. Webb, and P. J. Brown, "The effect of various denier capillary channel polymer fibers on the alignment of NHDF cells and type I collagen," Journal of Biomedical Materials Research Part A, vol. 95, no. 4, pp. 1194-1202, 2010.

[173] C. Hinüber, L. Häussler, R. Vogel, H. Brünig, and C. Werner, "Hollow poly (3-hydroxybutyrate) fibers produced by melt spinning," Macromolecular Materials and Engineering, vol. 295, no. 6, pp. 585-594, 2010.

[174] X. Wu, E. Rabkin-Aikawa, K. J. Guleserian, T. E. Perry, Y. Masuda, F. W. Sutherland, F. J. Schoen, J. E. Mayer Jr, and J. Bischoff, "Tissueengineered microvessels on three-dimensional biodegradable scaffolds using human endothelial progenitor cells," American Journal of Physiology-Heart and Circulatory Physiology, vol. 287, no. 2, pp. H480H487, 2004.

[175] J. Patrick, B. Krull, M. Garg, C. Mangun, J. Moore, N. Sottos, and S. White, "Robust sacrificial polymer templates for 3D interconnected microvasculature in fiber-reinforced composites," Composites Part A: Applied Science and Manufacturing, vol. 100, pp. 361-370, 2017.

[176] I. Kong, K. Tshai, and M. E. Hoque, "Manufacturing of natural fibrereinforced polymer composites by solvent casting method," Manufacturing of Natural Fibre Reinforced Polymer Composites, pp. 331-349: Springer, 2015.

[177] G. M. Gratson, M. Xu, and J. A. Lewis, "Microperiodic structures: Direct writing of three-dimensional webs," Nature, vol. 428, no. 6981 , pp. 386 , 2004. 
[178] D. Therriault, S. R. White, and J. A. Lewis, "Chaotic mixing in threedimensional microvascular networks fabricated by direct-write assembly," Nature materials, vol. 2, no. 4, pp. 265, 2003.

[179] A. S. Nain, C. Amon, and M. Sitti, "Three-dimensional nanoscale manipulation and manufacturing using proximal probes: controlled pulling of polymer micro/nanofibers." pp. 224-230.

[180] S. M. Berry, S. A. Harfenist, R. W. Cohn, and R. S. Keynton, "Characterization of micromanipulator-controlled dry spinning of micro-and sub-microscale polymer fibers," Journal of Micromechanics and Microengineering, vol. 16, no. 9, pp. 1825, 2006.

[181] S. M. Berry, S. Pabba, J. Crest, S. D. Cambron, G. H. McKinley, R. W. Cohn, and R. S. Keynton, "Characterization and modeling of direct-write fabrication of microscale polymer fibers," Polymer, vol. 52, no. 7, pp. 16541661, 2011.

[182] S. Z. Guo, F. Gosselin, N. Guerin, A. M. Lanouette, M. C. Heuzey, and D. Therriault, "Solvent-cast three-dimensional printing of multifunctional microsystems," Small, vol. 9, no. 24, pp. 4118-4122, 2013.

[183] S.-Z. Guo, M.-C. Heuzey, and D. Therriault, "Properties of polylactide inks for solvent-cast printing of three-dimensional freeform microstructures," Langmuir, vol. 30, no. 4, pp. 1142-1150, 2014.

[184] J. Kullenberg, F. Rosatini, G. Vozzi, F. Bianchi, A. Ahluwalia, and C. Domenici, "Optimization of PAM scaffolds for neural tissue engineering: preliminary study on an SH-SY5Y cell line," Tissue Engineering Part A, vol. 14, no. 6, pp. 1017-1023, 2008.

[185] A. S. Nain, J. A. Phillippi, M. Sitti, J. MacKrell, P. G. Campbell, and C. Amon, "Control of Cell Behavior by Aligned Micro/Nanofibrous Biomaterial Scaffolds Fabricated by Spinneret-Based Tunable Engineered Parameters (STEP) Technique," Small, vol. 4, no. 8, pp. 1153-1159, 2008.

[186] J. Wang, and A. S. Nain, "Suspended micro/nanofiber hierarchical biological scaffolds fabricated using non-electrospinning STEP technique," Langmuir, vol. 30, no. 45, pp. 13641-13649, 2014.

[187] A. C. Wan, M. F. Cutiongco, B. C. Tai, M. F. Leong, H. F. Lu, and E. K. Yim, "Fibers by interfacial polyelectrolyte complexation-processes, materials and applications," Materials Today, vol. 19, no. 8, pp. 437-450, 2016.

[188] C. Du, K. Narayanan, M. F. Leong, and A. C. Wan, "Induced pluripotent stem cell-derived hepatocytes and endothelial cells in multi-component hydrogel fibers for liver tissue engineering," Biomaterials, vol. 35, no. 23, pp. 6006-6014, 2014.

[189] H. F. Lu, K. Narayanan, S.-X. Lim, S. Gao, M. F. Leong, and A. C. Wan, "A 3D microfibrous scaffold for long-term human pluripotent stem cell selfrenewal under chemically defined conditions," Biomaterials, vol. 33, no. 8, pp. 2419-2430, 2012.

[190] T. C. Lim, M. F. Leong, H. Lu, C. Du, S. Gao, A. C. Wan, and J. Y. Ying, "Follicular dermal papilla structures by organization of epithelial and 
mesenchymal cells in interfacial polyelectrolyte complex fibers," Biomaterials, vol. 34, no. 29, pp. 7064-7072, 2013.

[191] H. M. Golecki, H. Yuan, C. Glavin, B. Potter, M. R. Badrossamay, J. A. Goss, M. D. Phillips, and K. K. Parker, "Effect of solvent evaporation on fiber morphology in rotary jet spinning," Langmuir, vol. 30, no. 44, pp. 13369-13374, 2014.

[192] M. R. Badrossamay, H. A. Mcllwee, J. A. Goss, and K. K. Parker, "Nanofiber assembly by rotary jet-spinning," Nano letters, vol. 10, no. 6, pp. 2257-2261, 2010.

[193] P. Qiu, and C. Mao, "Biomimetic branched hollow fibers templated by selfassembled fibrous polyvinylpyrrolidone structures in aqueous solution," ACS nano, vol. 4, no. 3, pp. 1573-1579, 2010.

[194] H.-W. Wang, C.-W. Cheng, C.-W. Li, and G.-J. Wang, "Fabrication of pillared PLGA microvessel scaffold using femtosecond laser ablation." pp. 33-39.

[195] H. K. Wong, C. R. I. Lam, F. Wen, S. K. M. Chong, N. S. Tan, C. Jerry, M. Pal, and L. P. Tan, "Novel method to improve vascularization of tissue engineered constructs with biodegradable fibers," Biofabrication, vol. 8 , no. 1, pp. 015004, 2016.

[196] Z.-M. Huang, Y. Zhang, S. Ramakrishna, and C. Lim, "Electrospinning and mechanical characterization of gelatin nanofibers," Polymer, vol. 45, no. 15, pp. 5361-5368, 2004.

[197] G. H. McKinley, and A. Tripathi, "How to extract the Newtonian viscosity from capillary breakup measurements in a filament rheometer," Journal of Rheology, vol. 44, no. 3, pp. 653-670, 2000.

[198] M. I. Kolte, and P. Szabo, "Capillary thinning of polymeric filaments," Journal of Rheology, vol. 43, no. 3, pp. 609-625, 1999.

[199] A. Tripathi, P. Whittingstall, and G. H. McKinley, "Using filament stretching rheometry to predict strand formation and "processability" in adhesives and other non-Newtonian fluids," Rheologica Acta, vol. 39, no. 4, pp. 321337, 2000.

[200] M. Stelter, G. Brenn, A. Yarin, R. Singh, and F. Durst, "Validation and application of a novel elongational device for polymer solutions," Journal of Rheology, vol. 44, no. 3, pp. 595-616, 2000.

[201] R. J. Klebe, K. L. Bentley, and R. C. Schoen, "Adhesive substrates for fibronectin," Journal of cellular physiology, vol. 109, no. 3, pp. 481-488, 1981.

[202] H. Yuan, S. D. Cambron, and R. S. Keynton, "Prescribed 3-D Direct Writing of Suspended Micron/Sub-Micron Scale Fiber Structures Via a Robotic Dispensing System," JoVE (Journal of Visualized Experiments), no. 100, pp. e52834, 2015.

[203] G. Stainsby, "Viscosity of dilute gelatin solutions," Nature, vol. 169, no. 4303, pp. 662, 1952.

[204] K. Sisson, C. Zhang, M. C. Farach-Carson, D. B. Chase, and J. F. Rabolt, "Evaluation of cross-linking methods for electrospun gelatin on cell growth and viability," Biomacromolecules, vol. 10, no. 7, pp. 1675-1680, 2009. 
[205] Y. Wan, W. Chen, J. Yang, J. Bei, and S. Wang, "Biodegradable poly (Llactide)-poly (ethylene glycol) multiblock copolymer: synthesis and evaluation of cell affinity," Biomaterials, vol. 24, no. 13, pp. 2195-2203, 2003.

[206] M. Giannotta, M. Trani, and E. Dejana, "VE-cadherin and endothelial adherens junctions: active guardians of vascular integrity," Developmental cell, vol. 26, no. 5, pp. 441-454, 2013.

[207] L. M. Goddard, and L. M. Iruela-Arispe, "Cellular and molecular regulation of vascular permeability," Thrombosis and haemostasis, vol. 109, no. 03, pp. 407-415, 2013.

[208] J. W. Nichol, and A. Khademhosseini, "Modular tissue engineering: engineering biological tissues from the bottom up," Soft matter, vol. 5, no. 7, pp. 1312-1319, 2009.

[209] C. Kirkpatrick, M. Otto, T. Van Kooten, V. Krump, J. Kriegsmann, and F. Bittinger, "Endothelial cell cultures as a tool in biomaterial research," Journal of Materials Science: Materials in Medicine, vol. 10, no. 10-11, pp. 589-594, 1999.

[210] O. Traub, and B. C. Berk, "Laminar shear stress: mechanisms by which endothelial cells transduce an atheroprotective force," Arteriosclerosis, thrombosis, and vascular biology, vol. 18, no. 5, pp. 677-685, 1998.

[211] L. Perry, S. Ben-Shaul, S. Landau, and S. Levenberg, "Co-culture systems for vasculogenesis," Vascularization for Tissue Engineering and Regenerative Medicine, pp. 1-29, 2017.

[212] G. M. Mitchell, and W. A. Morrison, "In vitro and in vivo approaches for pre-vascularization of 3-dimensional engineered tissues," Vascularization for Tissue Engineering and Regenerative Medicine, pp. 1-27, 2017. 


\title{
CURRICULUM VITA
}

\author{
Xiaoming Fan \\ Speed School of Engineering \\ University of Louisville, \\ Louisville, KY 40292 \\ 502-468-6922 \\ Xiaming.Fan@Louisville.edu
}

\section{EDUCATIION:}

08/14-12/19 Ph.D. in Mechanical Engineering (GPA: 3.89/4.00)

University of Louisville, Louisville, KY USA

Dissertation topic: Development and characterization of a biopolymer direct-write process for 3D microvascular structure formation

02/13-07/14 M.S. in Mechanical Engineering (GPA: 3.93/4.00)

University of Louisville, Louisville, KY USA

Thesis topic: Photomechanical actuation of liquid crystal nanotube

elastomers

08/10-05/12 Graduate Completion Certificate in Finance (GPA: 3.55/4.00)

Peking University, Beijing China

09/03-06/07 B.E. in Polymer Science and Engineering (GPA: 3.15/4.00)

Beijing University of Chemical Engineering, Beijing China

Thesis topic: Collagen Cerium Complexes' Preparation and the Research on Its Antimicrobial Property 


\section{EXPERIENCE:}

07/19- Present: GlobalFoundries, Malta, NY USA

02/13-06/19 University of Louisville, Louisville, KY USA

Research Assistant

03/08-07/12 China National Chemical Information Center Beijing, China

Deputy Manager of Oversea Sales (05/11-07/12)

Consultant (03/08-05/11)

\section{JOURNAL \& CONFERENCE PAPERS:}

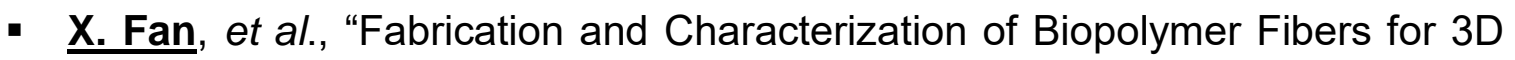
Oriented Microvascular Structures", Journal of Micromechanics and Microengineering, invited review paper, Mar 2018.

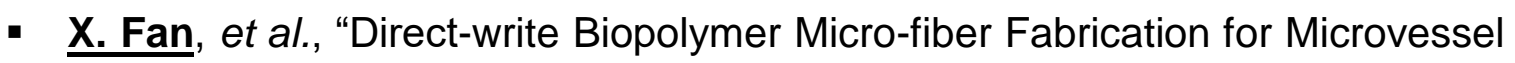
Formation", 2018 BMES Annual Meeting, Atlanta, GA, Oct 2018.

- X. Fan, et al., "Fabrication and Characterization of Biopolymer fibers for 3D Oriented Microvascular Structures by Direct-writing Technique", The 9th International Conference on Microtechnologies in Medicine and Biology (MMB 2018), Monterey, CA, Mar 2018.

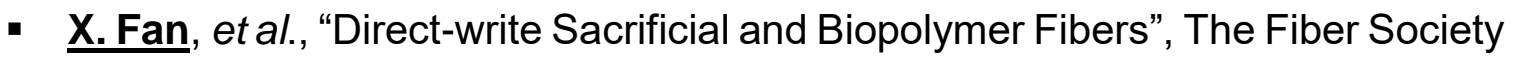
2017 Fall Meeting \& Technical Conference and International Symposium on Materials from Renewables (ISMR), Athens, GA, Nov 2017.

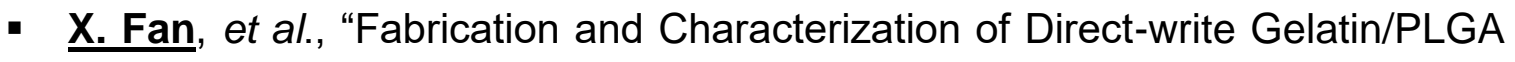
Fibers for Microvascular Angiogenesis", 2017 Advanced Biomanufacturing Conference (ABioM), Madison, WI, Aug 2017.

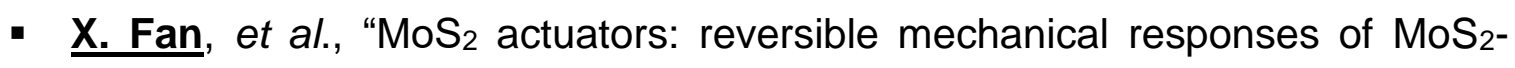
polymer nanocomposites to photons", Nanotechnology, 26.26 (2015):261001. 


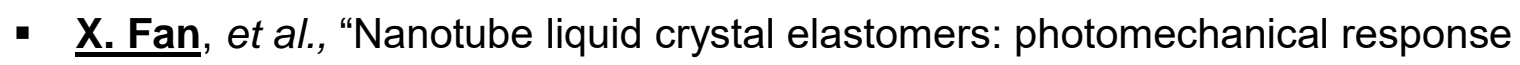
and flexible energy conversion of layered polymer composites", Nanotechnology, 25.35 (2014):355501. (Cover paper)

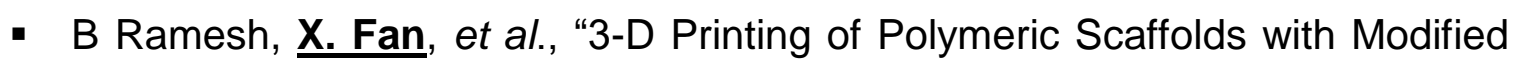
PLA-PEG Copolymers for Bioengineering Applications", Journal of Biomaterials Application, submitted, Oct 2018.

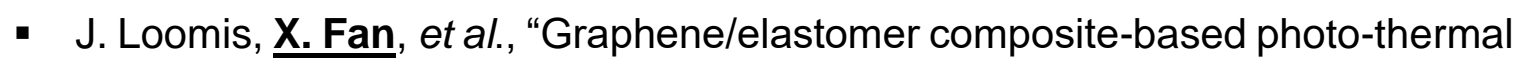
nanopositioners", Scientific Reports, 3 (2013):1900. 